\title{
Chemical Differentiation of Sugarcane Cultivars based on Volatile Profile and Chemometric Analysis
}

Pedro Silva $\dagger$, Jorge Freitas $\dagger$, Fernando M. Nunesț, José S. Câmara*, †, §

† CQM, Centro de Química da Madeira, Universidade da Madeira, Campus da Penteada, 9020-105 Funchal, Portugal

† CQ-VR, Centro de Química - Vila Real, Departamento de Química, Universidade de Trás-os-Montes e Alto Douro, 5001 - 801 Vila Real, Portugal

$\S$ Departamento de Química, Faculdade de Ciências Exactas e Engenharia, Universidade da Madeira; Campus da Penteada, $9020-105$ Funchal, Portugal

*Author to whom correspondence should be addressed

e-mail: jsc@staff.uma.pt (José S. Câmara)

Tel: +351 291705112; Fax: +351291705149 


\section{SUPPLEMENTARY INFORMATION}
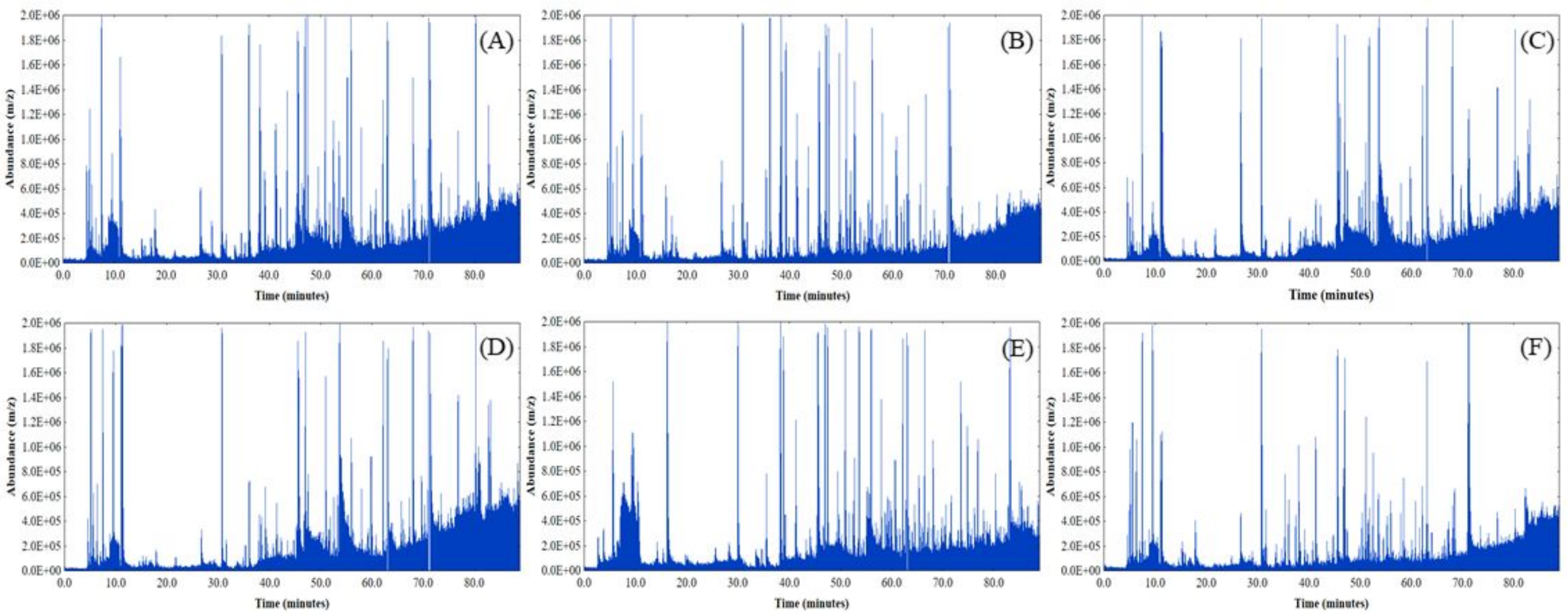

Figure S1. The typical GC-MS chromatograms for Amarela (A), Radiada (B), Roxa (C), Verde (D), Violeta (E) and Canica (F) sugarcane cultivars. 

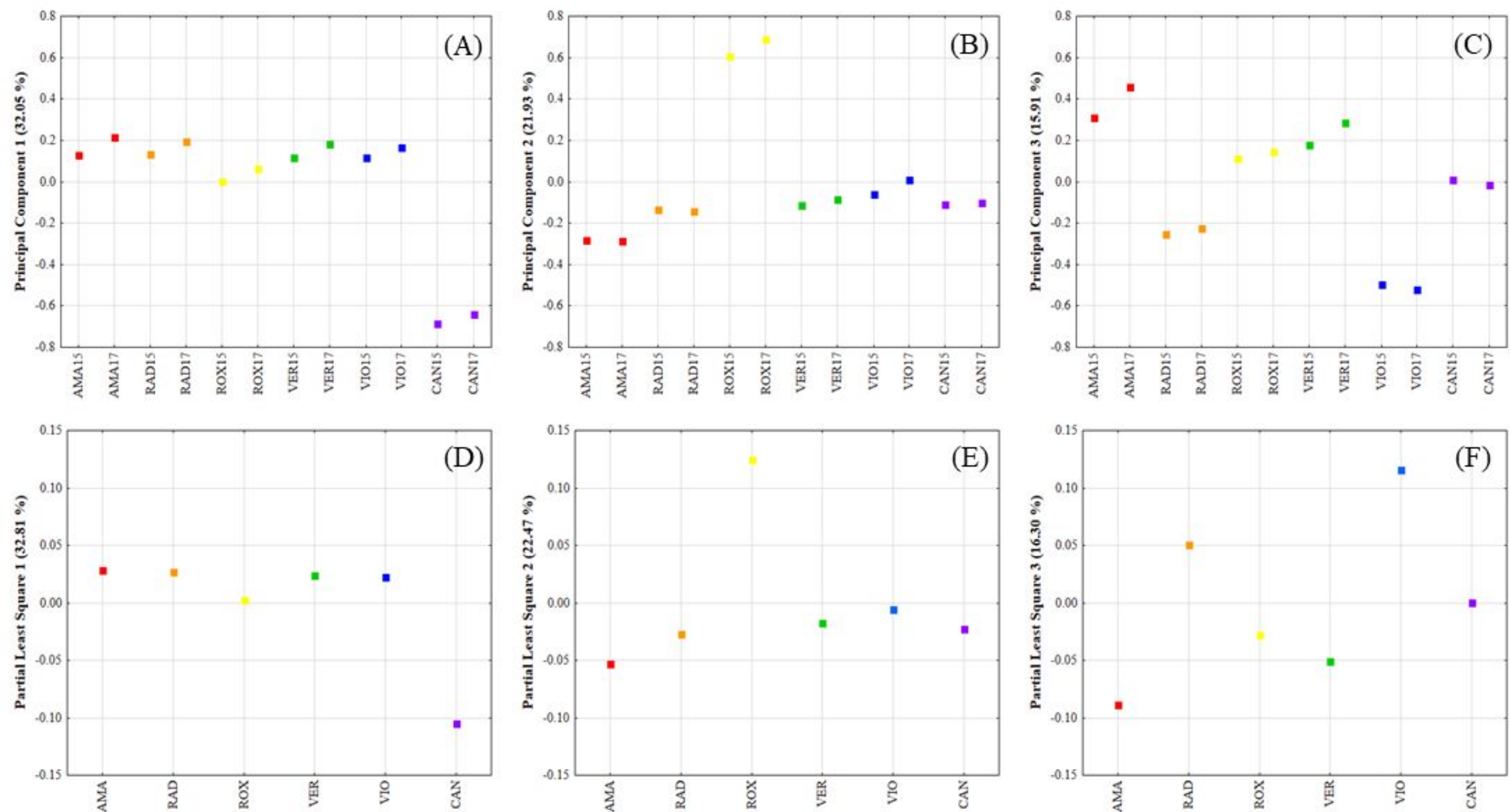

Figure S2. The PCA loadings line plot of all cultivar samples based on the selected 259 volatile organic compounds for PC1 (A), PC2 (B) and PC3 (C), and the PLS loadings line plot of all cultivar samples for PLS1 (D), PLS2 (E) and PLS3 (F). 

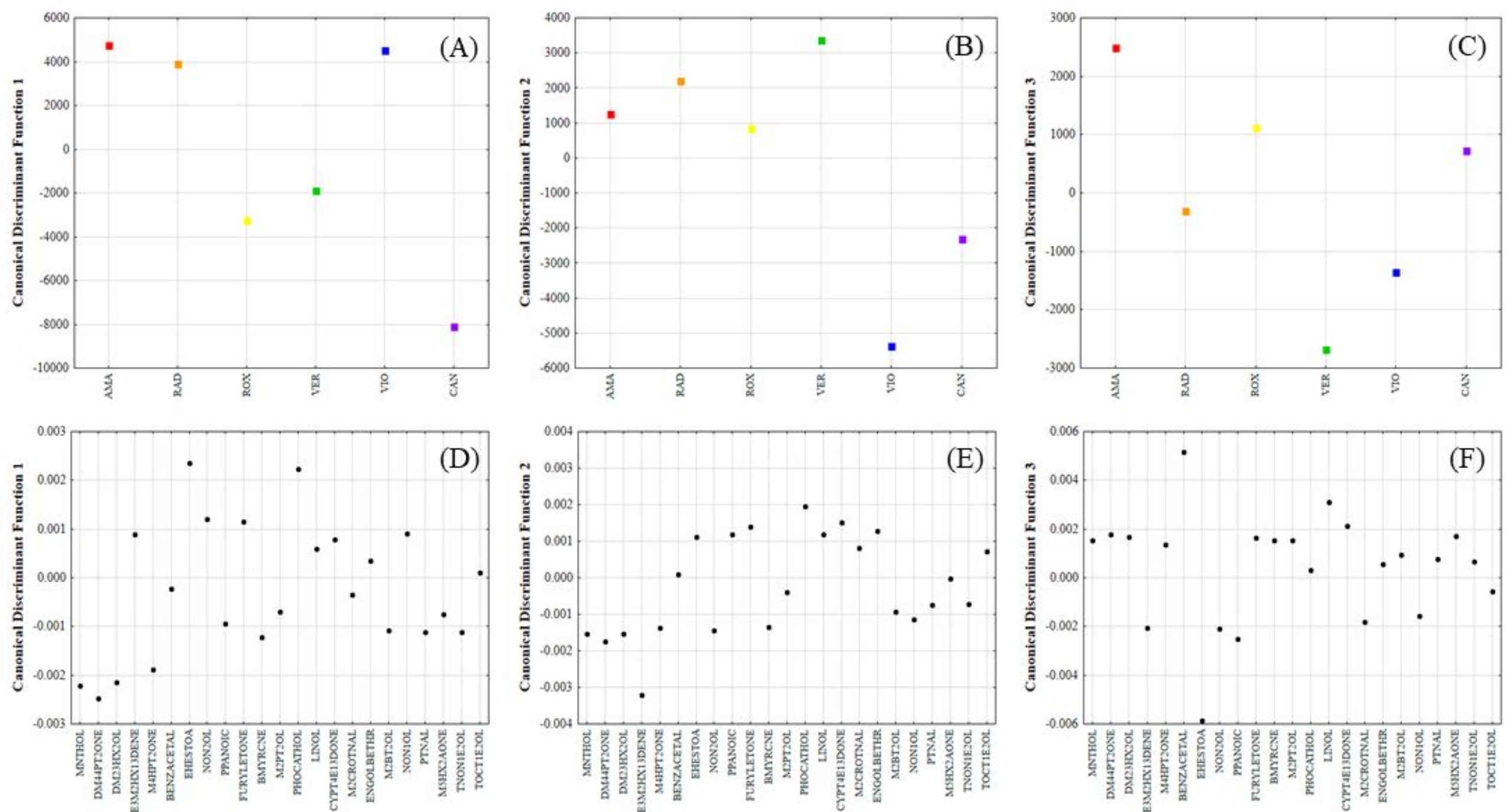

Figure S3. The LDA line plots of all cultivars based on the 23 most predictive volatile organic compounds for to CDF1 (A), CDF2 (B) and CDF3 (C), and LDA line plots of the 23 most predictive volatile organic compounds for CDF1 (D), CDF2 (E) and CDF3 (F). 

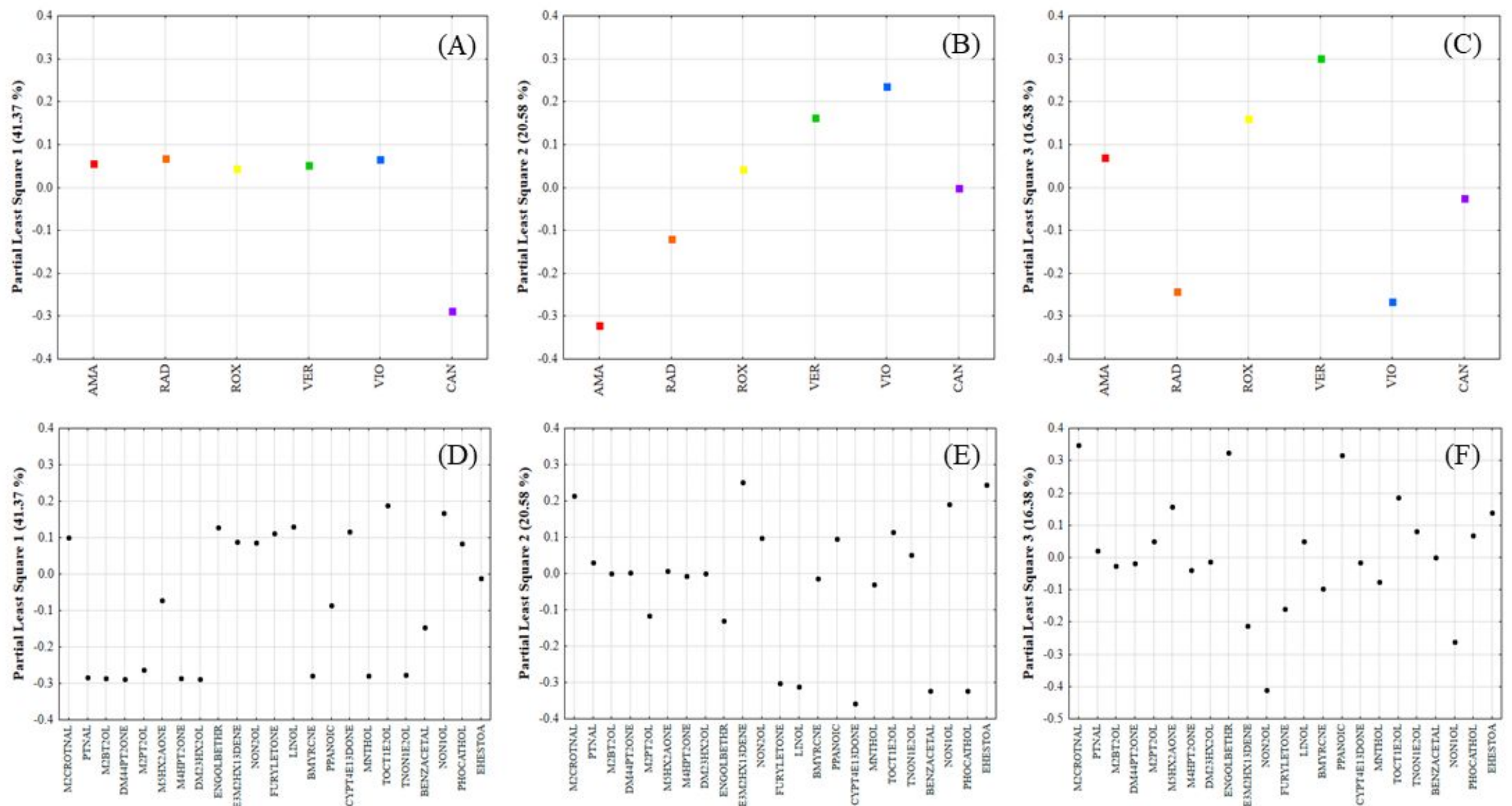

Figure S4. The PLS loadings line plots of all cultivar samples based on the 23 most predictive volatile organic compounds for PLS1 (A), PLS2 (B) and PLS3 (C), and the PLS loadings line plots of the 23 most predictive volatile organic compounds for PLS1 (D), PLS2 (E) and PLS3 (F). 

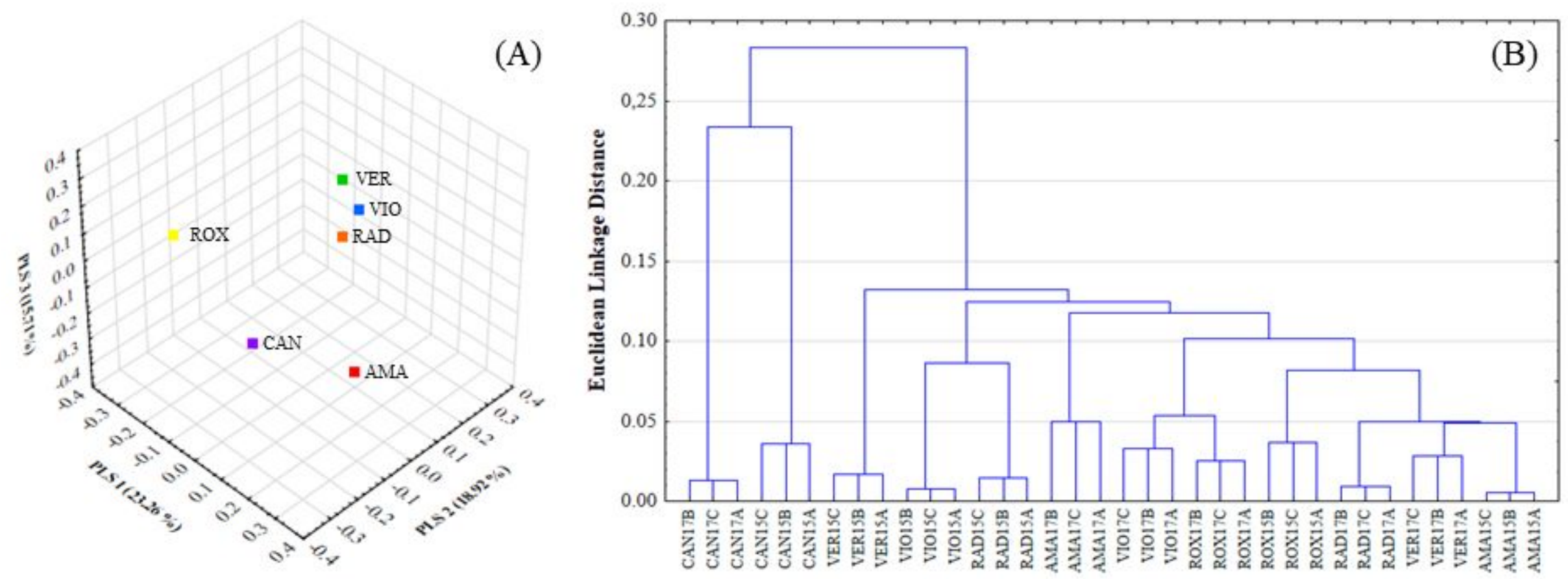

Figure S5. The PLS 3D plot (A) and HCA dendrogram (B) for results from the matrix reduction procedure based on the VIP scores. 
Table S1. ID replicate number, ID replicate code, ID sample code, ID variety code, variety empirical name, harvest year and geographical area of sugarcane samples.

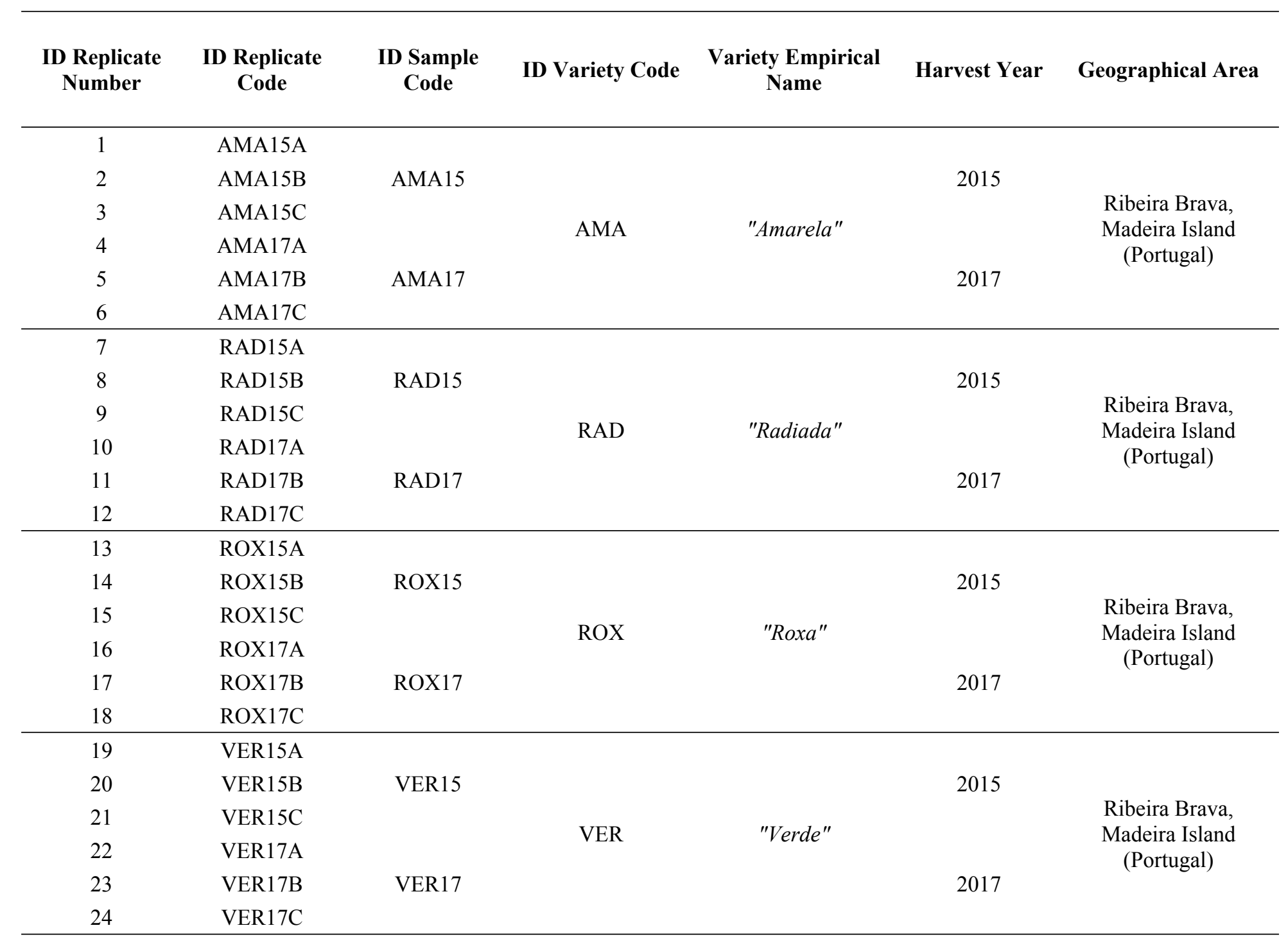




\begin{tabular}{|c|c|c|c|c|c|c|}
\hline 25 & VIO15A & & \multirow{6}{*}{ VIO } & \multirow{6}{*}{ "Violeta" } & \multirow{3}{*}{2015} & \multirow{6}{*}{$\begin{array}{l}\text { Ribeira Brava, } \\
\text { Madeira Island } \\
\text { (Portugal) }\end{array}$} \\
\hline 26 & VIO15B & VIO15 & & & & \\
\hline 27 & VIO15C & & & & & \\
\hline 28 & VIO17A & \multirow{3}{*}{ VIO17 } & & & \multirow{3}{*}{2017} & \\
\hline 29 & VIO17B & & & & & \\
\hline 30 & VIO17C & & & & & \\
\hline 31 & CAN15A & \multirow{4}{*}{ CAN15 } & \multirow{6}{*}{ CAN } & \multirow{6}{*}{ "Canica" } & \multirow{4}{*}{2015} & \multirow{6}{*}{$\begin{array}{l}\text { Machico, Madeira } \\
\text { Island (Portugal) }\end{array}$} \\
\hline 32 & CAN15B & & & & & \\
\hline 33 & CAN15C & & & & & \\
\hline 34 & CAN17A & & & & & \\
\hline 35 & CAN17B & \multirow[t]{2}{*}{ CAN17 } & & & \multirow[t]{2}{*}{2017} & \\
\hline 36 & CAN17C & & & & & \\
\hline
\end{tabular}

Table S2. ID number, retention time, main ions $(\mathrm{m} / \mathrm{z})$, target ion, match percent, identification type, IUPAC name, NIST database, abbreviation, CAS number, molecular formula and main chemical class of identified VOCs in sugarcane samples.

\begin{tabular}{|c|c|c|c|c|c|c|c|c|c|c|c|}
\hline $\begin{array}{c}\text { ID } \\
\text { Number }\end{array}$ & $\underset{(\mathrm{min})^{1}}{\mathrm{RT}}$ & $\mathbf{m} / \mathbf{z}$ & $\mathbf{T \mathbf { I } ^ { 2 }}$ & $\begin{array}{l}M^{3} \\
(\%)\end{array}$ & $\begin{array}{c}\text { ID } \\
\text { Type }^{4}\end{array}$ & IUPAC Name & NIST Database Name & Abbreviation & $\begin{array}{c}\text { CAS } \\
\text { Number }\end{array}$ & $\begin{array}{l}\text { Molecular } \\
\text { Formula }\end{array}$ & $\begin{array}{c}\text { Main } \\
\text { Chemical } \\
\text { Class }\end{array}$ \\
\hline 1 & 4.634 & $43 ; 42 ; 41$ & 43 & 87 & $\begin{array}{l}\text { RS; } \\
\text { MS }\end{array}$ & pentane & Pentane & PTANE & $109-66-0$ & C5H12 & Hydrocarbon \\
\hline 2 & 4.751 & $42 ; 55 ; 70$ & 42 & 84 & MS & pent-1-ene & 1-Pentene & PT1ENE & $109-67-1$ & $\mathrm{C} 5 \mathrm{H} 10$ & Hydrocarbon \\
\hline 3 & 4.786 & $43 ; 42 ; 41$ & 43 & 90 & MS & 2-methylpentane & 2-Methyl-Pentane & M2PTANE & $107-83-5$ & C6H14 & Hydrocarbon \\
\hline 5 & 5.046 & $59 ; 45 ; 74$ & 59 & 90 & $\begin{array}{l}\text { RS; } \\
\text { MS }\end{array}$ & ethoxyethane & Ethyl Ether & EETHR & $60-29-7$ & $\mathrm{C} 4 \mathrm{H} 10 \mathrm{O}$ & Ether \\
\hline 6 & 5.007 & $69 ; 41 ; 84$ & 69 & 82 & MS & (Z)-4-methylpent-2-ene & cis-4-Methyl-2-Pentene & CM4PT2ENE & $691-38-3$ & C6H12 & Hydrocarbon \\
\hline 7 & 5.179 & $67 ; 68 ; 53$ & 67 & 87 & MS & (3Z)-penta-1,3-diene & cis-Piperylene & CPIPERLNE & $1574-41-0$ & $\mathrm{C} 5 \mathrm{H} 8$ & Hydrocarbon \\
\hline
\end{tabular}




$\begin{array}{cccccc}8 & 5.196 & 56 ; 41 ; 55 & 56 & 91 & \mathrm{MS} \\ 9 & 5.306 & 41 ; 69 ; 84 & 41 & 80 & \mathrm{MS} \\ 10 & 5.352 & 43 ; 71 ; 57 & 71 & 79 & \mathrm{MS} \\ 11 & 5.392 & 67 ; 68 ; 53 & 67 & 91 & \mathrm{MS} \\ 12 & 5.467 & 47 ; 48 ; 45 & 47 & 84 & \mathrm{RS} \\ 13 & 5.526 & 67 ; 68 ; 53 & 67 & 78 & \mathrm{MS} \\ 14 & 5.566 & 43 ; 57 ; 71 & 57 & 90 & \mathrm{RS} \\ 15 & 5.641 & 59 ; 87 ; 57 & 59 & 78 & \mathrm{MS} \\ 16 & 5.672 & 44 ; 43 ; 42 & 42 & 80 & \mathrm{RS} \\ 17 & 6.200 & 47 ; 62 ; 45 & 47 & 90 & \mathrm{MS} \\ 18 & 6.343 & 43 ; 71 ; 70 & 71 & 91 & \mathrm{MS} \\ 19 & 7.002 & 43 ; 41 ; 57 & 57 & 90 & \mathrm{RS} ; \\ 20 & 7.020 & 58 ; 57 ; 59 & 58 & 87 & \mathrm{RS} ; \\ 21 & 7.044 & 68 ; 39 ; 40 & 68 & 91 & \mathrm{RS} ; \\ 22 & 7.202 & 43 ; 85 ; 57 & 43 & 91 & \mathrm{MS} \\ 23 & 7.400 & 43 ; 41 ; 72 & 72 & 90 & \mathrm{MS} \\ 24 & 7.480 & 43 ; 58 ; 39 & 58 & 77 & \mathrm{MS} \\ 25 & 7.865 & 43 ; 74 ; 59 & 74 & 85 & \mathrm{MS} \\ 26 & 8.054 & 56 ; 41 ; 55 & 56 & 87 & \mathrm{MS} \\ 27 & 8.215 & 41 ; 70 ; 39 & 70 & 82 & \mathrm{MS} \\ 28 & 8.431 & 43 ; 84 ; 85 & 84 & 83 & \mathrm{MS} \\ 32 & 8.536 & 43 ; 85 ; 41 & 85 & 90 & \mathrm{MS} \\ 31 & 9.455 & 82 ; 53 ; 81 & 82 & 98 & \mathrm{MS} \\ & 9.481 & 43 ; 70 ; 55 & 70 & 94 & \mathrm{MS} \\ & 9.816 & 43 ; 61 ; 70 & 61 & 86 & \mathrm{RS} ; \\ & & & & & \mathrm{MS}\end{array}$

2-methylpent-1-ene
(Z)-3-methylpent-2-ene
3-methylhexane
penta-1,4-diene
methanethiol
(3E)-penta-1,3-diene
heptane
2-ethoxy-2-methylpropane
acetaldehyde
methylsulfanylmethane
4-methylheptane
octane
propanal
furan
2,4-dimethylheptane
2-methylpropanal
propan-2-one
methyl acetate
2-methylhept-2-ene
(E)-but-2-enal
2,3-dimethylheptane
4-methyloctane
2-methylfuran
2,4-dimethylhept-1-ene
ethyl acetate

$\begin{array}{cccc}\text { M2PT1ENE } & 763-29-1 & \text { C6H12 } & \text { Hydrocarbon } \\ \text { CM3PT2ENE } & 922-62-3 & \text { C6H12 } & \text { Hydrocarbon } \\ \text { M3HXANE } & 589-34-4 & \text { C7H16 } & \text { Hydrocarbon } \\ \text { PT14DIENE } & 591-93-5 & \text { C5H8 } & \text { Hydrocarbon } \\ \text { METHIOL } & 74-93-1 & \text { CH4S } & \text { Sulfur } \\ \text { TPIPERLNE } & 2004-70-8 & \text { C5H8 } & \text { Hydrocarbon } \\ \text { HPTANE } & 142-82-5 & \text { C7H16 } & \text { Hydrocarbon } \\ \text { BEETHR } & 637-92-3 & \text { C6H14O } & \text { Ether } \\ \text { ETAL } & 75-07-0 & \text { C2H4O } & \text { Aldehyde } \\ \text { DMSULFI } & 75-18-3 & \text { C2H6S } & \text { Sulfur } \\ \text { M4HPTANE } & 589-53-7 & \text { C8H18 } & \text { Hydrocarbon } \\ \text { OCTANE } & 111-65-9 & \text { C8H18 } & \text { Hydrocarbon }\end{array}$

PPAL 123-38-6 C3H6O

FUR $\quad 110-00-9 \quad$ C4H4O

DM24HPTANE 2213-23-2 C9H20

MPPAL 78-84-2 C4H8O

PP2ONE 67-64-1 C3H6O

MESTAA 79-20-9 $\mathrm{C} 3 \mathrm{H} 6 \mathrm{O} 2$
2

141-78-6 C4H8O2

\section{Aldehyde}

Furan

Hydrocarbon Aldehyde

Ketone

Ester

Hydrocarbon

Aldehyde

Hydrocarbon

Hydrocarbon

Furan

Hydrocarbon

Ester
M2HPTENE

CROTNAL

DM23HPTANE

M4OCTANE

M2FUR

DM24HPT1ENE

EESTAA

627-97-4

4170-30-3

3074-71-3

2216-34-4

534-22-5

19549-87-

2,4-Dimethyl-1-Heptene

Ethyl Acetate 


\begin{tabular}{|c|c|c|c|c|c|c|c|c|c|c|c|}
\hline 33 & 10.146 & $43 ; 57 ; 85$ & 57 & 90 & $\begin{array}{l}\text { RS; } \\
\text { MS }\end{array}$ & nonane & Nonane & NNANE & $111-84-2$ & $\mathrm{C} 9 \mathrm{H} 20$ & Hydrocarbon \\
\hline 34 & 10.441 & $43 ; 72 ; 57$ & 72 & 80 & $\begin{array}{l}\text { RS; } \\
\text { MS }\end{array}$ & butan-2-one & 2-Butanone & BT2ONE & $78-93-3$ & $\mathrm{C} 4 \mathrm{H} 8 \mathrm{O}$ & Ketone \\
\hline 35 & 11.089 & $57 ; 41 ; 58$ & 57 & 90 & MS & 2-methylbutanal & 2-Methyl-Butanal & M2BTAL & $96-17-3$ & $\mathrm{C} 5 \mathrm{H} 10 \mathrm{O}$ & Aldehyde \\
\hline 36 & 11.353 & $44 ; 41,43$ & 44 & 96 & MS & 3-methylbutanal & 3-Methyl-Butanal & M3BTAL & $590-86-3$ & $\mathrm{C} 5 \mathrm{H} 10 \mathrm{O}$ & Aldehyde \\
\hline 37 & 12.143 & $31 ; 45 ; 46$ & 31 & 91 & $\begin{array}{l}\text { RS; } \\
\text { MS }\end{array}$ & ethanol & Ethyl Alcohol & ETOL & $64-17-5$ & $\mathrm{C} 2 \mathrm{H} 6 \mathrm{O}$ & Alcohol \\
\hline 38 & 12.684 & $78 ; 77 ; 52$ & 78 & 88 & $\begin{array}{l}\mathrm{RS} \\
\mathrm{MS}\end{array}$ & Benzene & Benzene & $\mathrm{BNZ}$ & $71-43-2$ & C6H6 & Benzene \\
\hline 39 & 13.280 & $57 ; 43 ; 41$ & 57 & 85 & MS & 3-ethylheptane & 3-Ethyl-Heptane & E3HPTANE & $\begin{array}{c}15869-80- \\
4\end{array}$ & $\mathrm{C} 9 \mathrm{H} 20$ & Hydrocarbon \\
\hline 40 & 13.526 & $81 ; 53 ; 96$ & 81 & 84 & MS & 2-ethylfuran & 2-Ethyl-Furan & E2FUR & $3208-16-0$ & C6H8O & Furan \\
\hline 41 & 13.837 & $57 ; 75 ; 102$ & 57 & 83 & MS & ethyl propanoate & Ethyl Propanoate & EESTPA & $105-37-3$ & $\mathrm{C} 5 \mathrm{H} 10 \mathrm{O} 2$ & Ester \\
\hline 42 & 14.389 & $43 ; 71 ; 41$ & 71 & 91 & MS & Ethyl 2-Methylpropanoate & Ethyl 2-Methylpropanoate & M2EESTPA & $97-62-1$ & $\mathrm{C} 6 \mathrm{H} 12 \mathrm{O} 2$ & Ester \\
\hline 43 & 14.495 & $55 ; 84 ; 39$ & 55 & 80 & MS & 2-methylbut-2-enal & 2-Methyl-crotonal & M2CROTNAL & $1115-11-3$ & $\mathrm{C} 5 \mathrm{H} 8 \mathrm{O}$ & Aldehyde \\
\hline 44 & 15.112 & $43 ; 61 ; 73$ & 61 & 82 & MS & Propyl acetate & Propyl acetate & PESTAA & $109-60-4$ & $\mathrm{C} 5 \mathrm{H} 10 \mathrm{O} 2$ & Ester \\
\hline 45 & 15.226 & $57 ; 86 ; 42$ & 57 & 83 & MS & pentan-3-one & 3-Pentanone & PT3ONE & $96-22-0$ & $\mathrm{C} 5 \mathrm{H} 10 \mathrm{O}$ & Ketone \\
\hline 46 & 15.407 & $44 ; 41 ; 58$ & 58 & 80 & $\begin{array}{l}\text { RS; } \\
\text { MS }\end{array}$ & pentanal & Pentanal & PTNAL & $110-62-3$ & $\mathrm{C} 5 \mathrm{H} 10 \mathrm{O}$ & Aldehyde \\
\hline 47 & 15.545 & $43 ; 86 ; 41$ & 86 & 80 & MS & butane-2,3-dione & 2,3-Butanedione & BT23DONE & $431-03-8$ & $\mathrm{C} 4 \mathrm{H} 6 \mathrm{O} 2$ & Ketone \\
\hline 48 & 16.362 & $41 ; 71 ; 57$ & 71 & 78 & MS & 2,3,4-trimethylhexane & 2,3,4-Trimethyl-Hexane & TM234HXANE & $921-47-1$ & $\mathrm{C} 9 \mathrm{H} 20$ & Hydrocarbon \\
\hline 49 & 16.699 & $57 ; 43 ; 41$ & 57 & 83 & $\begin{array}{l}\mathrm{RS} \\
\mathrm{MS}\end{array}$ & decane & Decane & DCANE & 124-18-5 & $\mathrm{C} 10 \mathrm{H} 22$ & Hydrocarbon \\
\hline 50 & 16.739 & $43 ; 57 ; 71$ & 57 & 84 & MS & 2,6-dimethylnonane & 2,6-Dimethyl-Nonane & DM26NNANE & $\begin{array}{c}17302-28- \\
2\end{array}$ & $\mathrm{C} 11 \mathrm{H} 24$ & Hydrocarbon \\
\hline 51 & 17.327 & $57 ; 43 ; 85$ & 57 & 84 & MS & 2,5-dimethylnonane & 2,5-Dimethyl-Nonane & DM25NNANE & $\begin{array}{c}17302-27- \\
1\end{array}$ & $\mathrm{C} 11 \mathrm{H} 24$ & Hydrocarbon \\
\hline 52 & 17.778 & $43 ; 58 ; 57$ & 58 & 83 & MS & 4-methylpentan-2-one & 4-Methyl-2-Pentanone & M4PT2ONE & $108-10-1$ & $\mathrm{C} 6 \mathrm{H} 12 \mathrm{O}$ & Ketone \\
\hline 53 & 18.238 & $59 ; 55 ; 73$ & 59 & 86 & MS & 2-methylbutan-2-ol & 2-Methy-2-Butanol & M2BT2OL & $75-85-4$ & C5H12O & Alcohol \\
\hline 54 & 18.558 & $43 ; 56 ; 73$ & 61 & 82 & MS & 2-methyl propyl acetate & 2-Methylpropyl Acetate & M2PESTAA & $110-19-0$ & $\mathrm{C} 6 \mathrm{H} 12 \mathrm{O} 2$ & Ester \\
\hline 55 & 18.705 & $93 ; 92 ; 77$ & 93 & 90 & MS & $\begin{array}{l}\text { 2,7,7-trimethyl-3- } \\
\text { oxatricyclo[4.1.1.02,4]octane }\end{array}$ & $\alpha$-Pinene & APNENE & $80-56-8$ & $\mathrm{C} 10 \mathrm{H} 16$ & Terpene \\
\hline 56 & 19.031 & $43 ; 57 ; 58$ & 57 & 80 & MS & 4,4-dimethylpentan-2-one & 4,4-Dimethyl-2-Pentanone & DM44PT2ONE & $590-50-1$ & $\mathrm{C} 7 \mathrm{H} 14 \mathrm{O}$ & Ketone \\
\hline 57 & 19.280 & $55 ; 84 ; 57$ & 55 & 89 & MS & pent-1-en-3-one & 1-Penten-3-one & PT1E3ONE & $1629-58-9$ & $\mathrm{C} 5 \mathrm{H} 8 \mathrm{O}$ & Ketone \\
\hline
\end{tabular}




\begin{tabular}{|c|c|c|c|c|c|c|c|c|c|c|c|}
\hline 58 & 20.416 & $57 ; 71 ; 43$ & 71 & 79 & MS & 5-butylnonane & 5-Butyl-Nonane & B5NONANE & $\begin{array}{l}17312-63- \\
9\end{array}$ & $\mathrm{C} 13 \mathrm{H} 28$ & Hydrocarbon \\
\hline 59 & 20.960 & $57 ; 43 ; 71$ & 57 & 83 & MS & 3,6-dimethyldecane & 3,6-Dimethyl-Decane & DM36DCANE & $\begin{array}{l}17312-53- \\
7\end{array}$ & $\mathrm{C} 12 \mathrm{H} 26$ & Hydrocarbon \\
\hline 60 & 21.599 & $71 ; 43 ; 59$ & 71 & 83 & MS & 2-methylbut-3-en-2-ol & 2-Methyl-3-Buten-2-ol & M3BT2OL & $115-18-4$ & $\mathrm{C} 5 \mathrm{H} 10 \mathrm{O}$ & Alcohol \\
\hline 61 & 22.378 & $57 ; 43 ; 41$ & 57 & 75 & MS & 5-methyldecane & 5-Methyl-Decane & M5DCANE & $\begin{array}{c}13151-35- \\
4\end{array}$ & $\mathrm{C} 11 \mathrm{H} 24$ & Hydrocarbon \\
\hline 62 & 22.856 & $43 ; 71 ; 51$ & 71 & 80 & MS & 4-methyldecane & 4-Methyl-Decane & M4DCANE & $2847-72-5$ & $\mathrm{C} 11 \mathrm{H} 24$ & Hydrocarbon \\
\hline 63 & 24.547 & $43 ; 57 ; 100$ & 57 & 90 & MS & pentane-2,3-dione & 2,3-Pentanedione & PTDONE & $600-14-6$ & $\mathrm{C} 5 \mathrm{H} 8 \mathrm{O} 2$ & Ketone \\
\hline 64 & 25.650 & $88 ; 43 ; 57$ & 88 & 90 & MS & methyl 3,3-dimethylbutanoate & $\begin{array}{c}\text { Methyl 3,3- } \\
\text { Dimethylbutanoate }\end{array}$ & DM33MESTBA & $\begin{array}{l}30540-29- \\
5\end{array}$ & $\mathrm{C} 7 \mathrm{H} 14 \mathrm{O} 2$ & Ester \\
\hline 65 & 26.145 & $43 ; 58 ; 100$ & 58 & 90 & MS & hexan-2-one & 2-Hexanone & HX2ONE & $591-78-6$ & C6H12O & Ketone \\
\hline 66 & 26.194 & $43 ; 71 ; 57$ & 71 & 81 & MS & 4-methylundecane & 4-Methyl-Undecane & M4UNCANE & 2980-69-0 & $\mathrm{C} 12 \mathrm{H} 26$ & Hydrocarbon \\
\hline 67 & 26.562 & $44 ; 56 ; 43$ & 56 & 94 & $\begin{array}{l}\mathrm{RS} \\
\mathrm{MS}\end{array}$ & hexanal & Hexanal & HXAL & $66-25-1$ & C6H12O & Aldehyde \\
\hline 68 & 28.766 & $43 ; 42 ; 41$ & 42 & 87 & $\begin{array}{l}\mathrm{RS} \\
\mathrm{MS}\end{array}$ & 2-methylpropan-1-ol & 2-Methyl-1-Propanol & M2PP1OL & $78-83-1$ & $\mathrm{C} 4 \mathrm{H} 10 \mathrm{O}$ & Alcohol \\
\hline 69 & 29.460 & $59 ; 45 ; 87$ & 59 & 80 & MS & 2-methylpentan-2-ol & 2-Methyl-2-Pentanol & M2PT2OL & $590-36-3$ & C6H14O & Alcohol \\
\hline 70 & 30.332 & $91 ; 106 ; 51$ & 91 & 91 & MS & ethylbenzene & Ethyl-Benzene & ETBNZ & $100-41-4$ & $\mathrm{C} 8 \mathrm{H} 10$ & Benzene \\
\hline 71 & 30.481 & $43 ; 70 ; 55$ & 70 & 77 & MS & 3-methylbutyl acetate & 3-Methylbutyl Acetate & M3BESTAA & $123-92-2$ & $\mathrm{C} 7 \mathrm{H} 14 \mathrm{O} 2$ & Ester \\
\hline 72 & 30.970 & $\begin{array}{l}91 ; 106 \\
105\end{array}$ & 91 & 97 & MS & 1,2-dimethylbenzene & o-Xylene & OXYLNE & $95-47-6$ & $\mathrm{C} 8 \mathrm{H} 10$ & Benzene \\
\hline 73 & 31.184 & $45 ; 55 ; 73$ & 45 & 82 & MS & pentan-2-ol & 2-Pentanol & PT2OL & $6032-29-7$ & $\mathrm{C} 5 \mathrm{H} 12 \mathrm{O}$ & Alcohol \\
\hline 74 & 31.578 & $\begin{array}{l}91 ; 106 \\
105\end{array}$ & 91 & 97 & MS & 1,4-dimethylbenzene & p-Xylene & PXYLNE & $106-42-3$ & $\mathrm{C} 8 \mathrm{H} 10$ & Benzene \\
\hline 75 & 32.219 & $43 ; 58 ; 57$ & 58 & 82 & MS & 5-methylhexan-2-one & 5-Methyl-2-Hexanone & M5HX2AONE & $110-12-3$ & $\mathrm{C} 7 \mathrm{H} 14 \mathrm{O}$ & Ketone \\
\hline 76 & 31.552 & $87 ; 43 ; 102$ & 87 & 83 & MS & methyl 2-ethylpentanoate & Methyl 2-Ethylpentanoate & E2MESTPTA & $816-16-0$ & $\mathrm{C} 8 \mathrm{H} 16 \mathrm{O} 2$ & Ester \\
\hline 77 & 33.137 & $56 ; 31 ; 41$ & 56 & 84 & $\begin{array}{l}\mathrm{RS} \\
\mathrm{MS}\end{array}$ & butan-1-ol & 1-Butanol & BT1OL & $71-36-3$ & $\mathrm{C} 4 \mathrm{H} 10 \mathrm{O}$ & Alcohol \\
\hline 78 & 33.392 & $93 ; 91 ; 77$ & 93 & 91 & MS & $\begin{array}{l}\text { (5S)-2-methyl-5-propan-2- } \\
\text { ylcyclohexa-1,3-diene }\end{array}$ & $\alpha$-Phellandrene & APHELDNE & $99-83-2$ & $\mathrm{C} 10 \mathrm{H} 16$ & Terpene \\
\hline 79 & 34.134 & $59 ; 73 ; 31$ & 59 & 91 & MS & 2-methylpentan-3-ol & 2-Methyl-3-Pentanol & M2PT3OL & $565-67-3$ & C6H14O & Alcohol \\
\hline 80 & 34.482 & 57; 41: 43 & 57 & 84 & MS & pent-1-en-3-ol & 1-Penten-3-ol & PT1E3OL & $616-25-1$ & $\mathrm{C} 5 \mathrm{H} 10 \mathrm{O}$ & Alcohol \\
\hline 81 & 34.773 & $\begin{array}{l}121 ; 93 \\
136\end{array}$ & 121 & 98 & MS & $\begin{array}{l}\text { 1-methyl-4-propan-2-ylcyclohexa- } \\
\text { 1,3-diene }\end{array}$ & $\alpha$-Terpinene & ATERPNNE & $99-86-5$ & $\mathrm{C} 10 \mathrm{H} 16$ & Terpene \\
\hline
\end{tabular}




$\begin{array}{lccccc}82 & 35.372 & 55 ; 56 ; 73 & 55 & 80 & \text { MS } \\ 83 & 35.689 & 91 ; 106 ; & 91 & 93 & \text { MS } \\ 84 & 35.845 & 43 ; 58 ; 71 & 58 & 92 & \text { MS } \\ 85 & 36.061 & 44 ; 70 ; 43 & 70 & 90 & \text { RS; } \\ 86 & 36.508 & 68 ; 93 ; 67 & 68 & 94 & \text { RS; } \\ 87 & 37.160 & 59 ; 55 ; 73 & 59 & 79 & \text { MS } \\ 88 & 37.200 & 93 ; 77 ; 91 & 93 & 94 & \text { MS } \\ 89 & 37.292 & 43 ; 81 ; 93 & 81 & 88 & \text { MS } \\ 90 & 37.530 & 95 ; 138 ; 67 & 95 & 84 & \text { MS } \\ 91 & 37.772 & 43 ; 58 ; 85 & 58 & 89 & \text { MS } \\ 92 & 37.856 & 45 ; 41 ; 56 & 45 & 78 & \text { MS } \\ 93 & 38.574 & 57 ; 41 ; 56 & 57 & 83 & \text { RS; } \\ 94 & 38.644 & 55 ; 42 ; 70 & 55 & 90 & \text { MS } \\ 95 & 38.715 & 105 ; 120 ; & 105 & 90 & \text { MS } \\ 96 & 38.948 & 105 ; 120 ; & 120 & 85 & \text { MS } \\ 97 & 39.430 & 45 ; 41 ; 69 & 45 & 90 & \text { MS } \\ 98 & 39.509 & 59 ; 31 ; 45 & 59 & 90 & \text { MS } \\ 99 & 40.058 & 43 ; 58 ; 71 & 58 & 64 & \text { MS } \\ 100 & 40.291 & 105 ; 120 ; & 105 & 92 & \text { MS } \\ 101 & 40.443 & 43 ; 58 ; 85 & 58 & 90 & \text { MS } \\ 102 & 40.831 & 59 ; 55 ; 70 & 59 & 84 & \text { MS } \\ & 40.925 & 43 ; 57 ; 72 & 57 & 84 & \text { MS } \\ & 41.201 & 104 ; 103 ; & 104 & 95 & \text { MS }\end{array}$

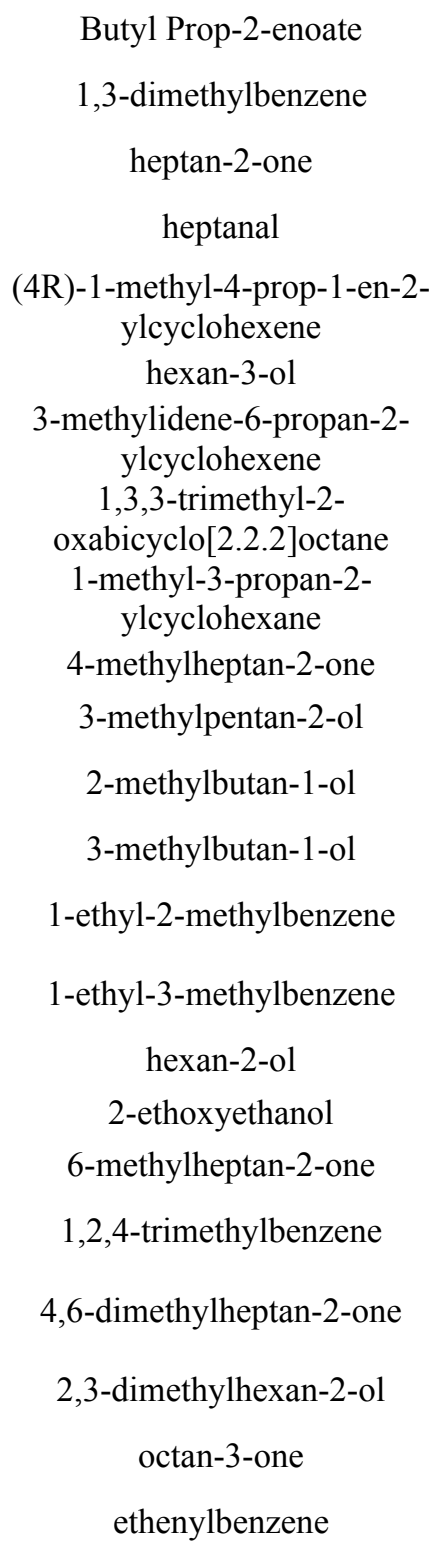

Butyl Prop-2-enoate
m-Xylene
2-Heptanone
Heptanal
D-Limonene
3-Hexanol
3-Phellandrene
Eucalyptol
m-Menthane
4-Methyl-2-Heptanone
3-Methyl-2-Pentanol
2-Methyl-1-Butanol
3-Methyl-1-Butanol
o-Ethyltoluene
m-Ethyltoluene
2-Hexanol
3-Octanone
6-Methyl-2-Heptanone
Ethylene Glycol Ethyl Ether
3imethyl-2-Heptanone
3imethyl-2-Hexanol
3tone

\begin{tabular}{|c|c|c|c|}
\hline BTESTPPEA & $141-32-2$ & $\mathrm{C} 7 \mathrm{H} 12 \mathrm{O} 2$ & Ester \\
\hline MXYLNE & $108-38-3$ & $\mathrm{C} 8 \mathrm{H} 10$ & Benzene \\
\hline HPT2ONE & $110-43-0$ & $\mathrm{C} 7 \mathrm{H} 14 \mathrm{O}$ & Ketone \\
\hline HPTAL & $111-71-7$ & $\mathrm{C} 7 \mathrm{H} 14 \mathrm{O}$ & Aldehyde \\
\hline DLIMNENE & $5989-27-5$ & $\mathrm{C} 10 \mathrm{H} 16$ & Terpene \\
\hline HX3OL & $623-37-0$ & $\mathrm{C} 6 \mathrm{H} 14 \mathrm{O}$ & Alcohol \\
\hline BPHELDNE & $555-10-2$ & $\mathrm{C} 10 \mathrm{H} 16$ & Terpene \\
\hline EUCPTOL & $470-82-6$ & $\mathrm{C} 10 \mathrm{H} 18 \mathrm{O}$ & Terpenoid \\
\hline MMTHANE & $\begin{array}{c}16580-24- \\
8\end{array}$ & $\mathrm{C} 10 \mathrm{H} 20$ & Terpenoid \\
\hline M4HPT2ONE & $6137-06-0$ & $\mathrm{C} 8 \mathrm{H} 16 \mathrm{O}$ & Ketone \\
\hline M2PT2OL & $565-60-6$ & $\mathrm{C} 6 \mathrm{H} 14 \mathrm{O}$ & Alcohol \\
\hline M2BT1OL & $137-32-6$ & $\mathrm{C} 5 \mathrm{H} 12 \mathrm{O}$ & Alcohol \\
\hline M3BT1OL & $123-51-3$ & $\mathrm{C} 5 \mathrm{H} 12 \mathrm{O}$ & Alcohol \\
\hline OETOLENE & $611-14-3$ & C9H12 & Benzene \\
\hline METOLENE & $620-14-4$ & C9H12 & Benzene \\
\hline HX2OL & $626-93-7$ & $\mathrm{C} 6 \mathrm{H} 14 \mathrm{O}$ & Alcohol \\
\hline ENGOLEETHR & $110-80-5$ & $\mathrm{C} 4 \mathrm{H} 10 \mathrm{O} 2$ & Ether \\
\hline M6HPT2ONE & $928-68-7$ & $\mathrm{C} 8 \mathrm{H} 16 \mathrm{O}$ & Ketone \\
\hline PSEUDCUMNE & $95-63-6$ & C9H12 & Benzene \\
\hline DM46HPT2ONE & $\begin{array}{c}19549-80- \\
5\end{array}$ & $\mathrm{C} 9 \mathrm{H} 18 \mathrm{O}$ & Ketone \\
\hline DM23HX2OL & $\begin{array}{c}19550-03- \\
9\end{array}$ & $\mathrm{C} 8 \mathrm{H} 18 \mathrm{O}$ & Alcohol \\
\hline OCT3ONE & $106-68-3$ & $\mathrm{C} 8 \mathrm{H} 16 \mathrm{O}$ & Ketone \\
\hline STYNE & $100-42-5$ & $\mathrm{C} 8 \mathrm{H} 8$ & Benzene \\
\hline
\end{tabular}




\begin{tabular}{|c|c|c|c|c|c|c|c|c|c|c|c|}
\hline 105 & 41.225 & $43 ; 57 ; 71$ & 71 & 84 & MS & 5-methylheptan-2-one & 5-Methyl-2-Heptanone & M5HPTAONE & $\begin{array}{c}18217-12- \\
4\end{array}$ & $\mathrm{C} 8 \mathrm{H} 16 \mathrm{O}$ & Ketone \\
\hline 106 & 41.401 & $42 ; 55 ; 70$ & 55 & 88 & $\begin{array}{l}\mathrm{RS} \\
\mathrm{MS}\end{array}$ & pentan-1-ol & 1-Pentanol & PT1OL & $71-41-0$ & $\mathrm{C} 5 \mathrm{H} 12 \mathrm{O}$ & Alcohol \\
\hline 107 & 41.548 & $\begin{array}{l}105 ; 120 \\
\quad 91\end{array}$ & 120 & 83 & MS & 1-ethyl-4-methylbenzene & p-Ethyltoluene & PETOLENE & $622-96-8$ & С9H12 & Benzene \\
\hline 108 & 42.183 & $\begin{array}{c}119 ; 134 \\
91\end{array}$ & 119 & 95 & MS & 1-methyl-4-propan-2-ylbenzene & p-Cymene & PCYMNE & $99-87-6$ & C10H14 & Terpenoid \\
\hline 109 & 42.429 & $\begin{array}{l}105 ; 120 \\
\quad 77\end{array}$ & 105 & 95 & MS & 1,3,5-trimethylbenzene & Mesitylene & MESTLNE & $108-67-8$ & С9H12 & Benzene \\
\hline 110 & 42.623 & $87 ; 45 ; 43$ & 87 & 82 & MS & 4-methylheptan-4-ol & 4-Methyl-4-Heptanol & M4HPT4OL & $598-01-6$ & $\mathrm{C} 8 \mathrm{H} 18 \mathrm{O}$ & Alcohol \\
\hline 111 & 43.151 & $55 ; 73 ; 43$ & 55 & 80 & MS & heptan-4-ol & 4-Heptanol & HPT4OL & $589-55-9$ & $\mathrm{C} 7 \mathrm{H} 16 \mathrm{O}$ & Alcohol \\
\hline 112 & 43.220 & $43 ; 58 ; 71$ & 58 & 94 & $\begin{array}{l}\text { RS; } \\
\text { MS }\end{array}$ & octan-2-one & 2-Octanone & OCT2ONE & 111-13-7 & $\mathrm{C} 8 \mathrm{H} 16 \mathrm{O}$ & Ketone \\
\hline 113 & 43.513 & $43 ; 56 ; 84$ & 84 & 80 & $\begin{array}{l}\text { RS; } \\
\text { MS }\end{array}$ & octanal & Octanal & OCTAL & $124-13-0$ & $\mathrm{C} 8 \mathrm{H} 16 \mathrm{O}$ & Aldehyde \\
\hline 114 & 44.239 & $45 ; 43 ; 88$ & 88 & 83 & MS & 3-hydroxybutan-2-one & 3-Hydroxy-2-Butanone & HXY3BT2ONE & $513-86-0$ & $\mathrm{C} 4 \mathrm{H} 8 \mathrm{O} 2$ & Ketone \\
\hline 115 & 44.294 & $55 ; 70 ; 43$ & 55 & 79 & MS & oct-1-en-3-one & 1-Octen-3-one & OCT3E1ONE & $4312-99-6$ & $\mathrm{C} 8 \mathrm{H} 14 \mathrm{O}$ & Ketone \\
\hline 116 & 44.946 & $57 ; 41 ; 39$ & 57 & 78 & MS & (E)-pent-2-en-1-ol & trans-2-Pentenol & TPT1E2OL & $1576-96-1$ & C5H10O & Alcohol \\
\hline 117 & 45.122 & $82 ; 56 ; 41$ & 82 & 76 & MS & 2,2,6-trimethylcyclohexan-1-one & $\begin{array}{l}\text { 2,2,6-Trimethyl- } \\
\text { Cyclohexanone }\end{array}$ & TM226CHXONE & $2408-37-9$ & С9H16O & Ketone \\
\hline 118 & 45.290 & $45 ; 55 ; 83$ & 55 & 79 & MS & heptan-2-ol & 2-Heptanol & HPT2OL & $543-49-7$ & C7H16O & Alcohol \\
\hline 119 & 45.426 & $57 ; 41 ; 39$ & 57 & 86 & MS & (Z)-pent-2-en-1-ol & cis-2-Pentenol & CPT1E2OL & $1576-95-0$ & $\mathrm{C} 5 \mathrm{H} 10 \mathrm{O}$ & Alcohol \\
\hline 120 & 45.615 & $43 ; 31 ; 74$ & 74 & 80 & MS & 1-hydroxypropan-2-one & 1-Hydroxy-2-Propanone & HXY1PP2ONE & $116-09-6$ & $\mathrm{C} 3 \mathrm{H} 6 \mathrm{O} 2$ & Ketone \\
\hline 121 & 46.014 & $83 ; 55 ; 112$ & 83 & 83 & MS & 5-methylhex-4-en-3-one & 5-Methyl-4-Hexen-3-one & M5HX3E4ONE & $\begin{array}{c}13905-10- \\
7\end{array}$ & $\mathrm{C} 7 \mathrm{H} 12 \mathrm{O}$ & Ketone \\
\hline 122 & 46.336 & $\begin{array}{l}105 ; 120 \\
\quad 77\end{array}$ & 105 & 80 & MS & 1,2,3-trimethylbenzene & Hemimellitene & HEMIMLTNE & $526-73-8$ & $\mathrm{C} 9 \mathrm{H} 12$ & Benzene \\
\hline 123 & 46.355 & $43 ; 41 ; 69$ & 69 & 92 & MS & 6-methylhept-5-en-2-one & 6-Methyl-5-Hepten-2-one & M6HPT2E5ONE & 110-93-0 & C8H14O & Ketone \\
\hline 124 & 46.666 & $55 ; 42 ; 70$ & 55 & 83 & MS & pent-2-ene & 2-Pentene & PT2ENE & $109-68-2$ & C5H10 & Hydrocarbon \\
\hline 125 & 47.293 & $56 ; 43 ; 55$ & 56 & 84 & $\begin{array}{l}\text { RS; } \\
\text { MS }\end{array}$ & hexan-1-ol & 1-Hexanol & HX1OL & $111-27-3$ & $\mathrm{C} 6 \mathrm{H} 14 \mathrm{O}$ & Alcohol \\
\hline 126 & 47.639 & $82 ; 39 ; 54$ & 82 & 78 & MS & cyclopent-2-en-1-one & 2-Cyclopenten-1-one & CY2PT1EONE & $930-30-3$ & C5H6O & Ketone \\
\hline 127 & 48.262 & $67 ; 96 ; 53$ & 67 & 84 & MS & 2-methylcyclopent-2-en-1-one & $\begin{array}{l}\text { 2-Methyl-2-Cyclopenten-1- } \\
\text { one }\end{array}$ & M2CY2PT1EONE & $1120-73-6$ & C6H8O & Ketone \\
\hline
\end{tabular}




\begin{tabular}{|c|c|c|c|c|c|c|c|c|c|c|c|}
\hline 128 & 48.293 & $\begin{array}{l}117 ; 118 \\
\quad 91\end{array}$ & 117 & 80 & MS & [(E)-prop-1-enyl]benzene & $\beta$-Methy-Styrene & BMSTYNE & $637-50-3$ & $\mathrm{C} 9 \mathrm{H} 10$ & Benzene \\
\hline 129 & 48.694 & $69 ; 55 ; 73$ & 69 & 80 & MS & octan-4-ol & 4-Octanol & OCT4OL & $589-62-8$ & $\mathrm{C} 8 \mathrm{H} 18 \mathrm{O}$ & Alcohol \\
\hline 130 & 49.013 & $41 ; 67 ; 82$ & 67 & 80 & MS & (Z)-hex-3-en-1-ol & cis-3-Hexen-1-ol & CHX1E3OL & $928-96-1$ & С6H12O & Alcohol \\
\hline 131 & 49.099 & $58 ; 43 ; 71$ & 58 & 95 & MS & nonan-2-one & 2-Nonanone & NON2ONE & $821-55-6$ & $\mathrm{C} 9 \mathrm{H} 18 \mathrm{O}$ & Ketone \\
\hline 132 & 49.328 & $57 ; 41 ; 43$ & 57 & 93 & $\begin{array}{l}\mathrm{RS} \\
\mathrm{MS}\end{array}$ & nonanal & Nonanal & NONAL & $124-19-6$ & С9H18O & Aldehyde \\
\hline 33 & 49.978 & $57 ; 45 ; 41$ & 59 & 78 & MS & 2-butoxyethanol & Ethylene Glycol Butyl Ether & ENGOLBETHR & $111-76-2$ & $\mathrm{C} 6 \mathrm{H} 14 \mathrm{O} 2$ & Ether \\
\hline 134 & 50.046 & $83 ; 55 ; 41$ & 83 & 83 & MS & 1-ethyl-1-methylcyclopentane & $\begin{array}{l}\text { 1-Ethyl-1-Methyl- } \\
\text { Cyclopentane }\end{array}$ & E1M1CYPTANE & $3726-47-4$ & $\mathrm{C} 8 \mathrm{H} 16$ & Hydrocarbon \\
\hline 135 & 50.253 & $55 ; 43 ; 111$ & 55 & 93 & MS & oct-3-en-2-one & 3-Octen-2-one & OCT2E3ONE & $1669-44-9$ & $\mathrm{C} 8 \mathrm{H} 14 \mathrm{O}$ & Ketone \\
\hline 136 & 50.612 & $45 ; 55 ; 41$ & 45 & 86 & MS & octan-2-ol & 2-Octanol & OCT2OL & $123-96-6$ & $\mathrm{C} 8 \mathrm{H} 18 \mathrm{O}$ & Alcohol \\
\hline 137 & 50.812 & $67 ; 95 ; 124$ & 67 & 82 & MS & $\begin{array}{c}\text { (3E)-3-ethyl-2-methylhexa-1,3- } \\
\text { diene }\end{array}$ & $\begin{array}{c}\text { 3-Ethyl-2-Methyl-1,3- } \\
\text { Hexadiene }\end{array}$ & E3M2HX13DENE & $\begin{array}{c}61142-36- \\
7\end{array}$ & $\mathrm{C} 9 \mathrm{H} 16$ & Terpene \\
\hline 138 & 51.178 & $175 ; 57 ; 41$ & 175 & 93 & MS & 1,3-ditert-butylbenzene & $\begin{array}{l}\text { 1,3-Bis(1,1-Dimethylethyl)- } \\
\text { Benzene }\end{array}$ & BI13DME11BNZ & $1014-60-4$ & $\mathrm{C} 14 \mathrm{H} 22$ & Benzene \\
\hline 139 & 51.323 & $41 ; 55 ; 70$ & 41 & 83 & MS & (E)-oct-2-enal & trans-2-Octenal & TOCT2EAL & $2548-87-0$ & $\mathrm{C} 8 \mathrm{H} 14 \mathrm{O}$ & Aldehyde \\
\hline 140 & 51.677 & $\begin{array}{l}117 ; 132 \\
115\end{array}$ & 117 & 80 & MS & 1-ethenyl-3-ethylbenzene & m-Ethyl-Styrene & MESTYNE & $7525-62-4$ & $\mathrm{C} 10 \mathrm{H} 12$ & Benzene \\
\hline 141 & 51.733 & $43 ; 58 ; 71$ & 58 & 82 & MS & 5-methylheptan-2-one & 5-Methyl-2-Heptanone & M5HPT2ONE & $\begin{array}{c}18217-12- \\
4\end{array}$ & $\mathrm{C} 8 \mathrm{H} 16 \mathrm{O}$ & Ketone \\
\hline 142 & 51.915 & $58 ; 43 ; 71$ & 58 & 83 & MS & decan-2-one & 2-Decanone & DEC2ONE & $693-54-9$ & $\mathrm{C} 10 \mathrm{H} 20 \mathrm{O}$ & Ketone \\
\hline 143 & 52.160 & $57 ; 43 ; 72$ & 57 & 86 & MS & oct-1-en-3-ol & 1-Octen-3-ol & OCT3E1OL & $3391-86-4$ & $\mathrm{C} 8 \mathrm{H} 16 \mathrm{O}$ & Alcohol \\
\hline 144 & 52.338 & $81 ; 110 ; 53$ & 81 & 86 & MS & (2E,4E)-hepta-2,4-dienal & trans,trans-2,4-Heptadienal & TTHPT22DEAL & $4313-03-5$ & $\mathrm{C} 7 \mathrm{H} 10 \mathrm{O}$ & Aldehyde \\
\hline 145 & 52.406 & $70 ; 56 ; 55$ & 70 & 83 & MS & heptan-1-ol & 1-Heptanol & HPT1OL & $111-70-6$ & $\mathrm{C} 7 \mathrm{H} 16 \mathrm{O}$ & Alcohol \\
\hline 146 & 52.697 & $81 ; 67 ; 68$ & 81 & 83 & MS & $(3 Z, 5 Z)$-octa-3,5-diene & cis,cis-3,5-Octadiene & CCOCT35DENE & $7348-80-3$ & $\mathrm{C} 8 \mathrm{H} 14$ & Hydrocarbon \\
\hline 147 & 52.959 & $43 ; 102 ; 59$ & 102 & 75 & MS & methyl 2-oxopropanoate & Methyl 2-Oxopropanoate & OM2ESTPPA & $600-22-6$ & $\mathrm{C} 4 \mathrm{H} 6 \mathrm{O} 3$ & Ester \\
\hline 148 & 53.063 & $59 ; 43 ; 55$ & 59 & 77 & MS & 2,6-dimethyloct-7-en-2-ol & Dihydromyrcenol & DHMYRCNOL & $\begin{array}{c}18479-58- \\
8\end{array}$ & $\mathrm{C} 10 \mathrm{H} 20 \mathrm{O}$ & Terpenoid \\
\hline 149 & 53.170 & $112 ; 69 ; 41$ & 112 & 97 & MS & $\begin{array}{c}\text { (2R)-5-methyl-2-propan-2- } \\
\text { ylcyclohexan-1-one }\end{array}$ & trans-Menthone & TMTHONE & $\begin{array}{l}10458-14- \\
7\end{array}$ & $\mathrm{C} 10 \mathrm{H} 18 \mathrm{O}$ & Terpenoid \\
\hline 150 & 53.292 & $96 ; 95 ; 39$ & 96 & 97 & $\begin{array}{l}\mathrm{RS} \\
\mathrm{MS}\end{array}$ & 2-furanaldehyde & Furfural & FURAL & $98-01-1$ & $\mathrm{C} 5 \mathrm{H} 4 \mathrm{O} 2$ & Furan \\
\hline 151 & 54.868 & $43 ; 45 ; 60$ & 60 & 91 & $\begin{array}{l}\mathrm{RS} \\
\mathrm{MS}\end{array}$ & Acetic Acid & Ethanoic Acid & ETNOIC & 64-19-7 & $\mathrm{C} 2 \mathrm{H} 4 \mathrm{O} 2$ & $\begin{array}{l}\text { Carboxylic } \\
\text { Acid }\end{array}$ \\
\hline
\end{tabular}




\begin{tabular}{|c|c|c|c|c|c|}
\hline 152 & 54.122 & $57 ; 41 ; 55$ & 57 & 57 & MS \\
\hline 153 & 54.884 & $57 ; 43 ; 55$ & 57 & 86 & $\begin{array}{l}\text { RS; } \\
\text { MS }\end{array}$ \\
\hline 154 & 54.886 & $81 ; 53 ; 126$ & 81 & 93 & MS \\
\hline 155 & 55.267 & $57 ; 41 ; 55$ & 57 & 91 & MS \\
\hline 156 & 55.575 & $45 ; 69 ; 55$ & 69 & 83 & MS \\
\hline 157 & 55.443 & $95 ; 110 ; 39$ & 95 & 90 & $\begin{array}{l}\text { RS; } \\
\text { MS }\end{array}$ \\
\hline 158 & 56.022 & $95 ; 43 ; 81$ & 95 & 84 & MS \\
\hline 159 & 56.414 & $\begin{array}{c}106 ; 105 \\
77\end{array}$ & 106 & 94 & $\begin{array}{l}\text { RS; } \\
\text { MS }\end{array}$ \\
\hline 160 & 56.771 & $81 ; 98 ; 52$ & 81 & 95 & MS \\
\hline 161 & 56.947 & $71 ; 43 ; 41$ & 71 & 90 & $\begin{array}{l}\text { RS; } \\
\text { MS }\end{array}$ \\
\hline 162 & 57.081 & $41 ; 93 ; 69$ & 93 & 80 & MS \\
\hline 163 & 57.530 & $56 ; 55 ; 70$ & 56 & 91 & $\begin{array}{l}\mathrm{RS} \\
\mathrm{MS}\end{array}$ \\
\hline 164 & 58.065 & $43 ; 93 ; 139$ & 93 & 95 & MS \\
\hline 165 & 58.338 & $74 ; 45 ; 73$ & 74 & 82 & MS \\
\hline 166 & 58.627 & $\begin{array}{c}45 ; 43 ; 57 \\
75\end{array}$ & 57 & 90 & MS \\
\hline 167 & 58.650 & $63 ; 78 ; 45$ & 63 & 82 & MS \\
\hline 168 & 58.917 & $95 ; 138 ; 67$ & 95 & 84 & MS \\
\hline 169 & 59.032 & $81 ; 55 ; 97$ & 81 & 90 & MS \\
\hline 170 & 59.265 & $95 ; 43 ; 93$ & 95 & 93 & MS \\
\hline 171 & 59.565 & $96 ; 42 ; 68$ & 96 & 86 & MS \\
\hline 172 & 59.658 & $71 ; 81 ; 95$ & 71 & 94 & $\begin{array}{l}\text { RS; } \\
\text { MS }\end{array}$ \\
\hline 173 & 59.677 & $58 ; 43 ; 71$ & 58 & 95 & MS \\
\hline
\end{tabular}

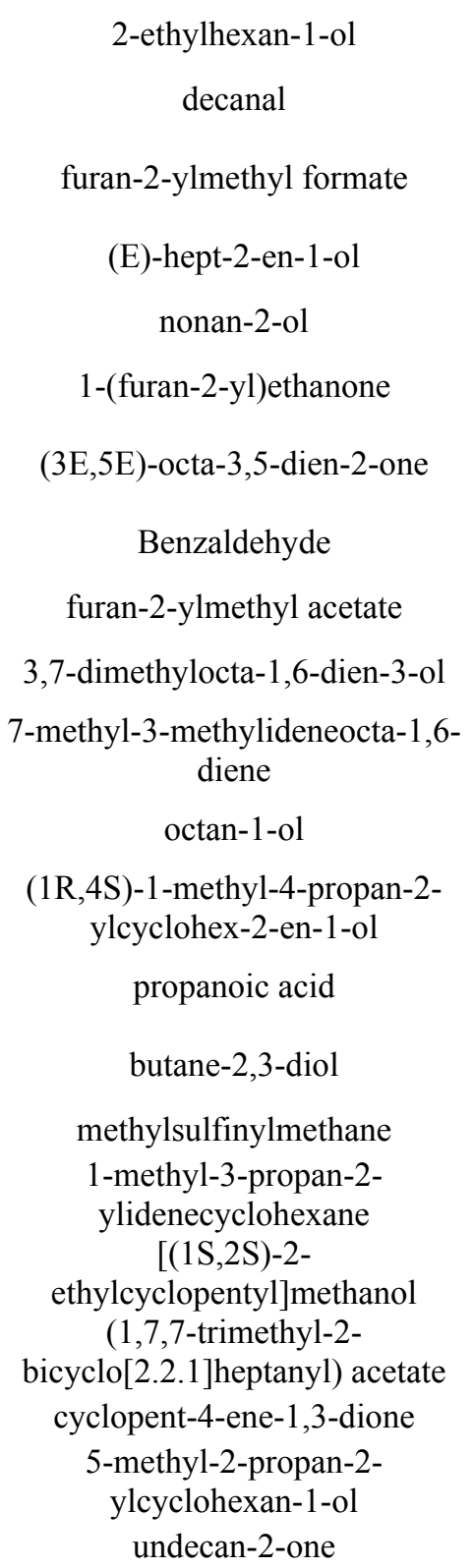

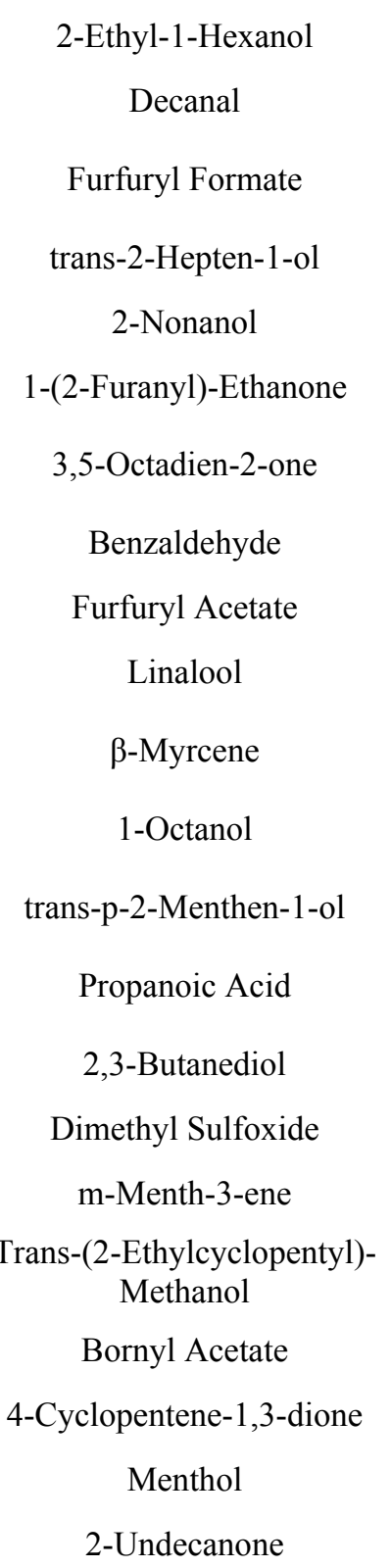

\begin{tabular}{|c|c|c|c|}
\hline E2HX1OL & $104-76-7$ & $\mathrm{C} 8 \mathrm{H} 18 \mathrm{O}$ & Alcohol \\
\hline DECAL & $112-31-2$ & $\mathrm{C} 10 \mathrm{H} 2 \mathrm{O}$ & Aldehyde \\
\hline FURYLFMTE & $\begin{array}{c}13493-97- \\
5\end{array}$ & $\mathrm{C} 6 \mathrm{H} 6 \mathrm{O} 3$ & Furan \\
\hline THPT1E2OL & $\begin{array}{c}33467-76- \\
4\end{array}$ & $\mathrm{C} 7 \mathrm{H} 14 \mathrm{O}$ & Alcohol \\
\hline NON2OL & $628-99-9$ & С9H20O & Alcohol \\
\hline FURYLETONE & $1192-62-7$ & $\mathrm{C} 6 \mathrm{H} 6 \mathrm{O} 2$ & Furan \\
\hline OCT35DE2ONE & $\begin{array}{c}38284-27- \\
4\end{array}$ & $\mathrm{C} 8 \mathrm{H} 12 \mathrm{O}$ & Ketone \\
\hline BENZAL & $100-52-7$ & $\mathrm{C} 7 \mathrm{H} 6 \mathrm{O}$ & Benzene \\
\hline FURYLACTE & $623-17-6$ & $\mathrm{C} 7 \mathrm{H} 8 \mathrm{O} 3$ & Furan \\
\hline LINOL & $78-70-6$ & $\mathrm{C} 10 \mathrm{H} 18 \mathrm{O}$ & Terpene \\
\hline BMYRCNE & $123-35-3$ & $\mathrm{C} 10 \mathrm{H} 16$ & Terpene \\
\hline OCT1OL & $111-87-5$ & $\mathrm{C} 8 \mathrm{H} 18 \mathrm{O}$ & Alcohol \\
\hline TMNTH1E2OL & $\begin{array}{c}29803-81- \\
4\end{array}$ & $\mathrm{C} 10 \mathrm{H} 18 \mathrm{O}$ & Terpenoid \\
\hline PPANOIC & $79-09-4$ & $\mathrm{C} 3 \mathrm{H} 6 \mathrm{O} 2$ & $\begin{array}{c}\text { Carboxylic } \\
\text { Acid }\end{array}$ \\
\hline BT23DIOL & $513-85-9$ & $\mathrm{C} 4 \mathrm{H} 10 \mathrm{O} 2$ & Alcohol \\
\hline DMSULFO & $67-68-5$ & $\mathrm{C} 2 \mathrm{H} 6 \mathrm{OS}$ & Sulfur \\
\hline MMTH3ENE & $\begin{array}{c}13828-34- \\
7\end{array}$ & $\mathrm{C} 10 \mathrm{H} 18$ & Terpene \\
\hline T2ЕСYРTMTOL & $\begin{array}{c}36258-08- \\
9\end{array}$ & $\mathrm{C} 8 \mathrm{H} 16 \mathrm{O}$ & Alcohol \\
\hline BORNYLACTE & $76-49-3$ & $\mathrm{C} 12 \mathrm{H} 20 \mathrm{O} 2$ & Ester \\
\hline CYPT4E13DONE & $930-60-9$ & $\mathrm{C} 5 \mathrm{H} 4 \mathrm{O} 2$ & Ketone \\
\hline MNTHOL & $89-78-1$ & $\mathrm{C} 10 \mathrm{H} 20 \mathrm{O}$ & Terpenoid \\
\hline UNDEC2ONE & $112-12-9$ & $\mathrm{C} 11 \mathrm{H} 22 \mathrm{O}$ & Ketone \\
\hline
\end{tabular}




\begin{tabular}{|c|c|c|c|c|c|c|c|c|c|c|c|}
\hline 174 & 59.851 & $\begin{array}{l}134 ; 78 \\
105\end{array}$ & 134 & 83 & MS & 2-(furan-2-yl)furan & 2-(2-Furanyl)-Furan & FURYLFUR & $5905-00-0$ & $\mathrm{C} 8 \mathrm{H} 6 \mathrm{O} 2$ & Furan \\
\hline 175 & 60.210 & $57 ; 41 ; 54$ & 57 & 89 & MS & (E)-oct-2-en-1-ol & trans-2-Octen-1-ol & TOCT1E2OL & $\begin{array}{c}18409-17- \\
1\end{array}$ & $\mathrm{C} 8 \mathrm{H} 16 \mathrm{O}$ & Alcohol \\
\hline 176 & 60.351 & $57 ; 41 ; 43$ & 57 & 82 & MS & (E)-non-2-en-1-ol & trans-2-Nonen-1-ol & TNON1E2OL & $\begin{array}{l}31502-14- \\
4\end{array}$ & С9H18O & Alcohol \\
\hline 177 & 60.719 & $45 ; 59 ; 72$ & 59 & 90 & MS & 2-(2-ethoxyethoxy)ethanol & $\begin{array}{l}\text { Diethylene Glycol Ethyl } \\
\text { Ether }\end{array}$ & DENGOLEETHR & $111-90-0$ & C6H14O3 & Ether \\
\hline 178 & 60.791 & $\begin{array}{l}109 ; 124 \\
\quad 81\end{array}$ & 109 & 82 & MS & $\begin{array}{c}\text { 3,5,5-trimethylcyclopent-2-en-1- } \\
\text { one }\end{array}$ & $\begin{array}{l}\text { 3,5,5-Trimethyl-2- } \\
\text { Cyclopenten-1-one }\end{array}$ & TM355CYPT1E2ONE & $\begin{array}{l}24156-95- \\
4\end{array}$ & $\mathrm{C} 8 \mathrm{H} 12 \mathrm{O}$ & Ketone \\
\hline 179 & 61.117 & $43 ; 93 ; 139$ & 93 & 89 & MS & 4-tert-butylcyclohex-2-en-1-ol & cis-p-2-Menthen-1-ol & CMNTH1E2OL & $\begin{array}{l}29803-82- \\
5\end{array}$ & $\mathrm{C} 10 \mathrm{H} 18 \mathrm{O}$ & Terpenoid \\
\hline 180 & 61.133 & $\begin{array}{l}137 ; 123 ; \\
152\end{array}$ & 137 & 91 & MS & $\begin{array}{l}\text { 2,6,6-trimethylcyclohexene-1- } \\
\text { carbaldehyde }\end{array}$ & $\beta$-Cyclocitral & BCYCITAL & $432-25-7$ & $\mathrm{C} 10 \mathrm{H} 16 \mathrm{O}$ & Terpenoid \\
\hline 181 & 62.190 & $91 ; 92 ; 120$ & 91 & 90 & MS & 2-phenylacetaldehyde & Benzeneacetaldehyde & BENZACETAL & $122-78-1$ & $\mathrm{C} 8 \mathrm{H} 8 \mathrm{O}$ & Benzene \\
\hline 182 & 62.284 & $56 ; 55 ; 43$ & 56 & 90 & MS & nonan-1-ol & 1-Nonanol & NON1OL & $143-08-8$ & С9H20O & Alcohol \\
\hline 183 & 62.615 & $98 ; 41 ; 81$ & 98 & 83 & $\begin{array}{l}\mathrm{RS} \\
\mathrm{MS}\end{array}$ & furan-2-ylmethanol & Furfuryl Alcohol & FUR2OL & $98-00-0$ & $\mathrm{C} 5 \mathrm{H} 6 \mathrm{O} 2$ & Furan \\
\hline 184 & 62.815 & $97 ; 79 ; 95$ & 97 & 90 & MS & $\begin{array}{l}\text { (5-ethylcyclopenten-1- } \\
\text { yl)methanol }\end{array}$ & Phoracanthol & PHOCATHOL & $\begin{array}{c}36431-59- \\
1\end{array}$ & $\mathrm{C} 8 \mathrm{H} 14 \mathrm{O}$ & Alcohol \\
\hline 185 & 63.440 & $55 ; 68 ; 81$ & 55 & 83 & MS & (Z)-non-3-en-1-ol & cis-3-Nonen-1-ol & ZNONE3OL & $\begin{array}{l}10340-23- \\
5\end{array}$ & С9H18O & Alcohol \\
\hline 186 & 63.478 & $84 ; 43 ; 139$ & 84 & 95 & MS & $\begin{array}{c}\text { (1R,6S)-3-methyl-6-propan-2- } \\
\text { ylcyclohex-2-en-1-ol }\end{array}$ & cis-Piperitol & CPIPETOL & $\begin{array}{l}16721-38- \\
3\end{array}$ & $\mathrm{C} 10 \mathrm{H} 18 \mathrm{O}$ & Terpenoid \\
\hline 187 & 63.636 & $\begin{array}{l}134 ; 119 ; \\
\quad 91\end{array}$ & 134 & 94 & MS & 1-ethenyl-4-methoxybenzene & p-Methoxy-Styrene & PMTXYESTYNE & $637-69-4$ & $\mathrm{C} 9 \mathrm{H} 10 \mathrm{O}$ & Benzene \\
\hline 188 & 63.727 & $55 ; 83 ; 41$ & 55 & 78 & MS & (2E)-3-ethylpenta-2,4-dien-1-ol & $\begin{array}{c}\text { (2Z)-3-Ethyl-2,4-Pentadien- } \\
1 \text {-ol }\end{array}$ & E3PT1DE24OL & $000-00-0$ & $\mathrm{C} 7 \mathrm{H} 12 \mathrm{O}$ & Alcohol \\
\hline 189 & 63.995 & $57 ; 55 ; 71$ & 57 & 94 & MS & 4-butoxybutan-1-ol & $\begin{array}{l}\text { Butylene Glycol Monobutyl } \\
\text { Ether }\end{array}$ & BNGOLBETHR & $4161-24-4$ & $\mathrm{C} 8 \mathrm{H} 18 \mathrm{O} 2$ & Ether \\
\hline 190 & 64.200 & $59 ; 93 ; 121$ & 59 & 85 & MS & $\begin{array}{l}\text { 2-[(1R)-4-methylcyclohex-3-en-1- } \\
\text { yl]propan-2-ol }\end{array}$ & $\alpha$-Terpineol & ATERPINOL & $98-55-5$ & $\mathrm{C} 10 \mathrm{H} 18 \mathrm{O}$ & Terpenoid \\
\hline 191 & 64.222 & $55 ; 56 ; 112$ & 55 & 89 & MS & cyclooctane-1,5-dione & 1,5-Cyclooctanedione & CYOCTD15ONE & $1489-74-3$ & $\mathrm{C} 8 \mathrm{H} 12 \mathrm{O} 2$ & Ketone \\
\hline 192 & 64.323 & $\begin{array}{c}121 ; 93 \\
136\end{array}$ & 121 & 90 & MS & $\begin{array}{c}\text { 1-methyl-4-propan-2-ylcyclohexa- } \\
\text { 1,4-diene }\end{array}$ & $\gamma$-Terpinene & GTERPINE & $99-85-4$ & $\mathrm{C} 10 \mathrm{H} 16$ & Terpene \\
\hline 193 & 64.718 & $57 ; 43 ; 41$ & 57 & 78 & MS & (E)-dec-2-en-1-ol & trans-2-Decenol & TDECE2OL & $\begin{array}{l}18409-18- \\
2\end{array}$ & $\mathrm{C} 10 \mathrm{H} 20 \mathrm{O}$ & Alcohol \\
\hline 194 & 64.731 & $72 ; 55 ; 45$ & 72 & 84 & MS & prop-2-enoic acid & 2-Propenoic Acid & PPE2NOIC & $79-10-7$ & $\mathrm{C} 3 \mathrm{H} 4 \mathrm{O} 2$ & $\begin{array}{l}\text { Carboxylic } \\
\text { Acid }\end{array}$ \\
\hline
\end{tabular}




\begin{tabular}{|c|c|c|c|c|c|}
\hline 195 & 64.863 & $45 ; 41 ; 43$ & 45 & 90 & Ms \\
\hline 196 & 65.068 & $\begin{array}{c}134 ; 133 \\
119\end{array}$ & 134 & 91 & Ms \\
\hline 197 & 65.330 & $\begin{array}{c}122 ; 121 \\
93\end{array}$ & 122 & 83 & Ms \\
\hline 198 & 65.475 & $55 ; 56 ; 41$ & 55 & 76 & Ms \\
\hline 199 & 65.578 & $41 ; 98 ; 69$ & 98 & 83 & 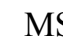 \\
\hline 200 & 65.918 & $67 ; 55 ; 81$ & 67 & 76 & Ms \\
\hline 201 & 66.228 & $84 ; 43 ; 139$ & 84 & 97 & 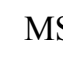 \\
\hline 202 & 66.391 & $\begin{array}{c}134 ; 133 \\
105\end{array}$ & 134 & 78 & Ms \\
\hline 203 & 66.510 & $\begin{array}{c}128 ; 129 \\
127\end{array}$ & 128 & 80 & S S \\
\hline 204 & 66.624 & $55 ; 43 ; 70$ & 70 & 91 & D \\
\hline 205 & 66.664 & $55 ; 70 ; 56$ & 55 & 96 & Ms \\
\hline 206 & 67.230 & $81 ; 47 ; 67$ & 81 & 87 & 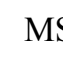 \\
\hline 207 & 67.532 & $98 ; 55 ; 42$ & 98 & 82 & Ms \\
\hline 208 & 67.847 & $67 ; 41 ; 54$ & 67 & 89 & Ms \\
\hline 209 & 68.067 & $68 ; 41 ; 81$ & 68 & 91 & $\mathrm{M}$ \\
\hline 210 & 68.915 & $57 ; 91 ; 148$ & 57 & 88 & Ms \\
\hline 211 & 69.029 & $107 ; 79 ; 77$ & 107 & 91 & Ms \\
\hline 212 & 69.080 & $81 ; 41 ; 67$ & 81 & 83 & 10 \\
\hline 213 & 69.212 & $70 ; 39 ; 41$ & 70 & 82 & Ms \\
\hline 214 & 69.476 & $73 ; 88 ; 41$ & 73 & 80 & Ms \\
\hline 215 & 69.769 & $112 ; 69 ; 41$ & 112 & 80 & Ms \\
\hline 216 & 70.128 & $\begin{array}{c}131 ; 132 \\
103\end{array}$ & 131 & 83 & Ms \\
\hline 217 & 70.617 & $60 ; 73 ; 43$ & 60 & 76 & $\mathrm{RS}$ \\
\hline
\end{tabular}

undecan-2-ol
3-ethylbenzaldehyde
3-hydroxybenzaldehyde
(E)-non-2-ene
(E)-2-methylpent-2-enal
(Z)-dec-5-en-1-ol
(1R,6R)-3-methyl-6-propan-2-
ylcyclohex-2-en-1-ol
4-ethylbenzaldehyde
naphthalene
decan-1-ol
cyclodecane
(2E,4E)-deca-2,4-dienal
cyclopentane-1,2-dione
cyclodecene
(Z)-dec-4-en-1-ol
1-phenylbutan-2-one
(2-methylphenyl)methanol
(2E,4Z)-deca-2,4-dienal
cyclohex-2-en-1-ol
2-ethylhexanoic acid
hethylcyclopentane-1,2-dione
(Ehenylprop-2-enal
(2)id
(2)

2-Undecanol
3-Ethyl-Benzaldehyde
3-Hydroxy-Benzaldehyde
trans-2-Nonene
2-Methyl-2-Pentenal
cis-5-Decenol
trans-Piperitol

4-Ethyl-Benzaldehyde

Naphthalene

1-Decanol

\section{Cyclodecane}

trans,trans-2,4-Decadienal

$$
\begin{gathered}
\text { 1,2-Cyclopentanedione } \\
\text { Cyclodecene } \\
\text { cis-4-Decenol }
\end{gathered}
$$

1-Phenyl-2-Butanone

$\alpha$-Methyl-Benzenemethanol

trans,cis-2,4-Decadienal

2-Cyclohexenol

2-Ethyl Hexanoic Acid

3-Methyl-1,2-

Cyclopentanedione

trans-Cinnamaldehyde

Heptanoic Acid

$\begin{array}{cccc}\text { UNDEC2OL } & 1653-30-1 & \mathrm{C} 11 \mathrm{H} 24 \mathrm{O} & \text { Alcohol } \\ \text { E3BNZAL } & 34246-54- & \mathrm{C} 9 \mathrm{H} 10 \mathrm{O} & \text { Benzene } \\ \text { HXY3BENZAL } & 100-83-4 & \mathrm{C} 7 \mathrm{H} 6 \mathrm{O} 2 & \text { Phenol } \\ \text { TNONENE } & 6434-78-2 & \mathrm{C} 9 \mathrm{H} 18 & \text { Hydrocarbon } \\ \text { M2PNTE2AL } & 623-36-9 & \mathrm{C} 6 \mathrm{H} 10 \mathrm{O} & \text { Aldehyde } \\ \text { CDEC5E1OL } & 51652-47- & \mathrm{C} 10 \mathrm{H} 20 \mathrm{O} & \text { Alcohol } \\ & 2 & & \\ \text { TPIPETOL } & 16721-39- & \mathrm{C} 10 \mathrm{H} 18 \mathrm{O} & \text { Terpenoid } \\ & 4 & & \\ \text { E4BNZAL } & 4748-78-1 & \mathrm{C} 9 \mathrm{H} 10 \mathrm{O} & \text { Benzene } \\ & & & \\ \text { NPHNE } & 91-20-3 & \mathrm{C} 10 \mathrm{H} 8 & \text { Naphthalene } \\ & 112-30-1 & \mathrm{C} 10 \mathrm{H} 22 \mathrm{O} & \text { Alcohol } \\ \text { DEC1OL } & 293-96-9 & \mathrm{C} 10 \mathrm{H} 20 & \text { Hydrocarbon } \\ \text { CDECANE } & 25152-84- & \mathrm{C} 10 \mathrm{H} 16 \mathrm{O} & \text { Aldehyde } \\ \text { TTDEC24DENAL } & 5 & & \text { Ketone } \\ \text { CPT12DONE } & 3008-40-0 & \mathrm{C} 5 \mathrm{H} 6 \mathrm{O} 2 & \text { Hydrocarbon } \\ \text { CDECENE } & 3618-12-0 & \mathrm{C} 10 \mathrm{H} 18 & \text { Alcohol } \\ \text { CDEC4E1OL } & 57074-37- & \mathrm{C} 10 \mathrm{H} 20 \mathrm{O} & \text { A } \\ \text { PHYLBT2ONE } & 1007-32-5 & \mathrm{C} 10 \mathrm{H} 12 \mathrm{O} & \text { Benzene } \\ \text { MBNZMTOL } & 98-85-1 & \mathrm{C} 8 \mathrm{H} 10 \mathrm{O} & \text { Benzene } \\ \text { TCDEC24DENAL } & 25152-83- & \mathrm{C} 10 \mathrm{H} 16 \mathrm{O} & \text { Aldehyde } \\ \text { CHEX2E1OL } & 822-67-3 & \mathrm{C} 6 \mathrm{H} 10 \mathrm{O} & \text { Alcohol } \\ \text { E2HEXOIC } & 149-57-5 & \mathrm{C} 8 \mathrm{H} 16 \mathrm{O} 2 & \text { Carboxylic } \\ \text { M3CPT12DONE } & 765-70-8 & \mathrm{C} 6 \mathrm{H} 8 \mathrm{O} 2 & \text { Ketone } \\ \text { TCINAMAL } & 14371-10- & \mathrm{C} 9 \mathrm{H} 8 \mathrm{O} & \text { Benzene } \\ \text { HEPTOIC } & 111-14-8 & \mathrm{C} 7 \mathrm{H} 14 \mathrm{O} 2 & \text { Carboxylic } \\ & & & \text { Acid }\end{array}$




\begin{tabular}{|c|c|c|c|c|c|}
\hline 218 & 71.026 & $109 ; 124,81$ & 109 & 92 & Ms \\
\hline 19 & 71.092 & $128 ; 57 ; 85$ & 128 & 78 & SS \\
\hline 20 & 71.409 & $\begin{array}{c}131 ; 132 \\
103\end{array}$ & 131 & 76 & Ms \\
\hline 21 & 71.559 & $79 ; 108 ; 77$ & 79 & 92 & M \\
\hline 22 & 71.682 & $\begin{array}{c}147 ; 148 \\
119\end{array}$ & 147 & 94 & $\Lambda$ \\
\hline 23 & 72.376 & $57 ; 70 ; 127$ & 57 & 87 & 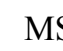 \\
\hline 24 & 72.907 & $91 ; 92 ; 122$ & 91 & 78 & $\mathrm{M}$ \\
\hline 25 & 72.929 & $42 ; 44,31$ & 42 & 78 & Ms \\
\hline 26 & 73.968 & $177 ; 43 ; 91$ & 177 & 97 & Ms \\
\hline 27 & 74.232 & $\begin{array}{c}124 ; 95 \\
107\end{array}$ & 124 & 50 & 1012 \\
\hline 228 & 74.246 & $88 ; 73 ; 101$ & 88 & 81 & Ms \\
\hline 29 & 74.422 & $55 ; 43 ; 69$ & 55 & 94 & Ms \\
\hline 230 & 74.631 & $\begin{array}{c}131 ; \\
103 ; 146\end{array}$ & 131 & 76 & Ms \\
\hline 31 & 74.950 & $126 ; 71 ; 43$ & 126 & 93 & $M S$ \\
\hline 232 & 75.126 & $94 ; 109 ; 66$ & 94 & 75 & S \\
\hline 233 & 75.164 & $41 ; 55 ; 83$ & 41 & 96 & $\mathrm{M}$ \\
\hline 234 & 75.978 & $98 ; 97 ; 53$ & 98 & 82 & IVI \\
\hline 235 & 76.247 & $94 ; 66 ; 65$ & 94 & 95 & MS \\
\hline 236 & 76.679 & $95 ; 126 ; 67$ & 95 & 75 & 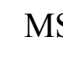 \\
\hline 237 & 77.333 & $57 ; 43 ; 71$ & 71 & 75 & 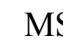 \\
\hline 238 & 77.352 & $88 ; 101 ; 43$ & 88 & 89 & IV \\
\hline 239 & 78.059 & $43 ; 103 ; 42$ & 103 & 85 & , \\
\hline 240 & 78.233 & $57 ; 85 ; 41$ & 57 & 83 & $\mathrm{M}$ \\
\hline
\end{tabular}

4-methoxyphenol
1,3-diazinane-2,4,5-trione
(Z)-3-phenylprop-2-enal
phenylmethanol
2,4,6-trimethylbenzaldehyde
2-ethylhexyl octanoate
2-phenylethanol
butane-1,4-diol
(E)-4-(2,6,6-trimethylcyclohexen-
1-yl)but-3-en-2-one
2-methylbenzene-1,4-diol
2-ethylheptanoic acid
dodecan-1-ol
(E)-4-phenylbut-3-en-2-one
3-hydroxy-2-methylpyran-4-one
1-(1H-pyrrol-2-yl)ethanone
(2E,4E)-deca-2,4-dien-1-ol
(1S,5R)-6,8-
dioxabicyclo[3.2.1]oct-2-en-4-one
phenol
1-(furan-2-yl)-2-hydroxyethanone
4,6-dimethyldodecane
ethyl decanoate
1-methylpiperidin-4-one
2,2-dimethylheptan-3-one

Mequinol
2,4,5-Trihydroxypyrimidine
cis-Cinnamaldehyde
Benzenemethanol
Mesitaldehyde
2-Ethylhexyl Octanoate
Benzeneethanol
1,4-Butanediol
B-Ionone
2-Methyl-1,4-Benzenediol
2-Ethyl Heptanoic Acid
1-Dodecanol
Benzalacetone
Maltol
2-Acetylpyrrole
2,4-Decadien-1-ol
Levoglucosenone
Phenol
Ethanone
1-(2-Furanyl)-Hydroxy-
Ethyl Decanoate
1-Methyl-4-Piperidinone
2,2-Dimethyl-3-Heptanone

\begin{tabular}{|c|c|c|c|}
\hline MEQNOL & $150-76-5$ & $\mathrm{C} 7 \mathrm{H} 8 \mathrm{O} 2$ & Phenol \\
\hline THDXYPYMNE & $67-52-7$ & $\mathrm{C} 4 \mathrm{H} 4 \mathrm{~N} 2 \mathrm{O} 3$ & Nitrogen \\
\hline CCINAMAL & $\begin{array}{c}57194-69- \\
1\end{array}$ & $\mathrm{C} 9 \mathrm{H} 8 \mathrm{O}$ & Benzene \\
\hline BENZMTOL & $100-51-6$ & $\mathrm{C} 7 \mathrm{H} 8 \mathrm{O}$ & Benzene \\
\hline MESIAL & $487-68-3$ & $\mathrm{C} 10 \mathrm{H} 12 \mathrm{O}$ & Benzene \\
\hline EHESTOA & $\begin{array}{c}63321-70- \\
0\end{array}$ & $\mathrm{C} 16 \mathrm{H} 32 \mathrm{O} 2$ & Ester \\
\hline BENZETOL & $60-12-8$ & $\mathrm{C} 8 \mathrm{H} 10 \mathrm{O}$ & Benzene \\
\hline BT14DIOL & $110-63-4$ & C4H10O2 & Alcohol \\
\hline BIONNE & $\begin{array}{c}14901-07- \\
6\end{array}$ & $\mathrm{C} 13 \mathrm{H} 20 \mathrm{O}$ & Terpenoid \\
\hline M2BNZ14DIOL & $95-71-6$ & $\mathrm{C} 7 \mathrm{H} 8 \mathrm{O} 2$ & Benzene \\
\hline EHPTOIC & $149-57-5$ & $\mathrm{C} 8 \mathrm{H} 16 \mathrm{O} 2$ & $\begin{array}{c}\text { Carboxylic } \\
\text { Acid }\end{array}$ \\
\hline DODEC1OL & $112-53-8$ & $\mathrm{C} 12 \mathrm{H} 26 \mathrm{O}$ & Alcohol \\
\hline BNZONE & $122-57-6$ & $\mathrm{C} 10 \mathrm{H} 10 \mathrm{O}$ & Benzene \\
\hline MALTOL & $118-71-8$ & $\mathrm{C} 6 \mathrm{H} 6 \mathrm{O} 3$ & Pyran \\
\hline ACTLPYROLE & $1072-83-9$ & C6H7NO & Nitrogen \\
\hline DEC24DIE1OL & $\begin{array}{c}14507-02- \\
9\end{array}$ & $\mathrm{C} 10 \mathrm{H} 18 \mathrm{O}$ & Alcohol \\
\hline LEVGLCONE & $\begin{array}{c}37112-31- \\
5\end{array}$ & $\mathrm{C} 6 \mathrm{H} 6 \mathrm{O} 3$ & Ketone \\
\hline PHEOL & $108-95-2$ & $\mathrm{C} 6 \mathrm{H} 6 \mathrm{O}$ & Phenol \\
\hline FURYLHXYEONE & $\begin{array}{c}17678-19- \\
2\end{array}$ & $\mathrm{C} 6 \mathrm{H} 6 \mathrm{O} 3$ & Furan \\
\hline DM45DODCANE & $\begin{array}{c}\text { 17312-63- } \\
9\end{array}$ & $\mathrm{C} 13 \mathrm{H} 28$ & Hydrocarbon \\
\hline EESDECA & $110-38-3$ & $\mathrm{C} 12 \mathrm{H} 24 \mathrm{O} 2$ & Ester \\
\hline M1PIP4DIONE & $1445-73-4$ & C6H11NO & Nitrogen \\
\hline DM22HPT3ONE & $\begin{array}{c}19078-97- \\
8\end{array}$ & С9H18O & Ketone \\
\hline
\end{tabular}




\begin{tabular}{|c|c|c|c|c|c|c|c|c|c|c|c|}
\hline 241 & 78.184 & $60 ; 73 ; 43$ & 60 & 80 & $\begin{array}{l}\mathrm{RS} \\
\mathrm{MS}\end{array}$ & octanoic acid & Octanoic Acid & OCTOIC & $124-07-2$ & $\mathrm{C} 8 \mathrm{H} 16 \mathrm{O} 2$ & $\begin{array}{l}\text { Carboxylic } \\
\text { Acid }\end{array}$ \\
\hline 242 & 79.187 & $31 ; 43 ; 42$ & 31 & 83 & MS & 1,3-dihydroxypropan-2-one & 1,3-Dihydroxy-2-Propanone & DHYPPAONE & $96-26-4$ & $\mathrm{C} 3 \mathrm{H} 6 \mathrm{O} 3$ & Ketone \\
\hline 243 & 80.862 & $94 ; 138 ; 77$ & 94 & 82 & MS & 2-phenoxyethanol & 2-Phenoxy-Ethanol & PHENXYOL & $122-99-6$ & $\mathrm{C} 8 \mathrm{H} 10 \mathrm{O} 2$ & Benzene \\
\hline 244 & 80.906 & $\begin{array}{l}134 ; 133 \\
105\end{array}$ & 134 & 78 & MS & benzene-1,3-dicarbaldehyde & Isophthalaldehyde & ISOPHTAL & $626-19-7$ & $\mathrm{C} 8 \mathrm{H} 6 \mathrm{O} 2$ & Benzene \\
\hline 245 & 81.320 & $55 ; 83 ; 69$ & 55 & 83 & MS & cyclotetradecane & Cyclotetradecane & CTTDECANE & $295-17-0$ & C14H28 & Hydrocarbon \\
\hline 246 & 81.611 & $60 ; 73 ; 57$ & 60 & 87 & $\begin{array}{l}\mathrm{RS} \\
\mathrm{MS}\end{array}$ & nonanoic acid & Nonanoic Acid & NONOIC & $112-05-0$ & $\mathrm{C} 9 \mathrm{H} 18 \mathrm{O} 2$ & $\begin{array}{l}\text { Carboxylic } \\
\text { Acid }\end{array}$ \\
\hline 247 & 81.853 & $\begin{array}{c}107 ; 122 \\
77\end{array}$ & 107 & 78 & MS & 2-ethylphenol & 2-Ethyl-Phenol & E2PHEOL & $90-00-6$ & $\mathrm{C} 8 \mathrm{H} 10 \mathrm{O}$ & Phenol \\
\hline 248 & 82.628 & $\begin{array}{l}135 ; 150 \\
77\end{array}$ & 135 & 88 & MS & 4-ethenyl-2-methoxyphenol & 2-Methoxy-4-Vinyl-Phenol & MXY2VYL4PHEOL & $7786-61-0$ & $\mathrm{C} 9 \mathrm{H} 10 \mathrm{O} 2$ & Phenol \\
\hline 249 & 82.875 & $82 ; 81 ; 54$ & 82 & 80 & MS & 4-Methyl-1H-imidazole & 4-Methylimidazole & M4IMDZOLE & $822-36-6$ & C4H6N2 & Nitrogen \\
\hline 250 & 84.049 & $\begin{array}{l}133 ; 105 \\
148\end{array}$ & 133 & 80 & MS & 1-tert-butyl-3-methylbenzene & m-tert-Butyl-Toluene & MTBTLNE & $1075-38-3$ & C11H16 & Benzene \\
\hline 251 & 84.676 & $\begin{array}{l}154 ; 139 \\
111\end{array}$ & 154 & 82 & MS & 2,6-dimethoxyphenol & Syringol & SYNGOL & $91-10-1$ & $\mathrm{C} 8 \mathrm{H} 10 \mathrm{O} 3$ & Phenol \\
\hline 252 & 84.890 & $60 ; 73 ; 41$ & 60 & 96 & $\begin{array}{l}\mathrm{RS} \\
\mathrm{MS}\end{array}$ & decanoic acid & Decanoic Acid & DECOIC & $334-48-5$ & $\mathrm{C} 10 \mathrm{H} 20 \mathrm{O} 2$ & $\begin{array}{l}\text { Carboxylic } \\
\text { Acid }\end{array}$ \\
\hline 253 & 85.090 & $43 ; 42 ; 144$ & 144 & 87 & MS & $\begin{array}{l}\text { 3,5-dihydroxy-6-methyl-2,3- } \\
\text { dihydropyran-4-one }\end{array}$ & $\begin{array}{l}\text { 3-Hydroxy-2,3-Dihydro- } \\
\text { Maltol }\end{array}$ & HX3DH23MALTOL & $\begin{array}{l}28564-83- \\
2\end{array}$ & C6H8O4 & Pyran \\
\hline 254 & 86.052 & $142 ; 43 ; 68$ & 142 & 84 & MS & $\begin{array}{c}\text { 3,5-dihydroxy-2-methylpyran-4- } \\
\text { one }\end{array}$ & 5-Hydroxy-Maltol & HX5MALTOL & $1073-96-7$ & C6H6O4 & Pyran \\
\hline 255 & 86.550 & $45 ; 89 ; 58$ & 89 & 82 & MS & $\begin{array}{c}2-[2-(2- \\
\text { hydroxyethoxy)ethoxy] ethanol }\end{array}$ & Triethylene Glycol & TETYNEGLOL & $112-27-6$ & C6H14O4 & Ether \\
\hline 256 & 88.144 & $\begin{array}{l}105 ; 122 \\
\quad 77\end{array}$ & 105 & 81 & $\begin{array}{l}\mathrm{RS} \\
\mathrm{MS}\end{array}$ & benzoic acid & Benzoic Acid & BNZOIC & $65-85-0$ & $\mathrm{C} 7 \mathrm{H} 6 \mathrm{O} 2$ & Benzene \\
\hline 257 & 89.084 & $\begin{array}{l}91 ; 120 \\
121\end{array}$ & 91 & 95 & MS & 2-methylbenzaldehyde & 2-Methyl-Benzaldehyde & M2BNZAL & $529-20-4$ & $\mathrm{C} 8 \mathrm{H} 8 \mathrm{O}$ & Benzene \\
\hline 258 & 89.701 & $60 ; 73 ; 43$ & 60 & 85 & MS & undecanoic acid & Undecanoic Acid & UNDECOIC & $112-37-8$ & $\mathrm{C} 11 \mathrm{H} 22 \mathrm{O} 2$ & $\begin{array}{l}\text { Carboxylic } \\
\text { Acid }\end{array}$ \\
\hline 259 & 90.106 & $95 ; 39 ; 68$ & 95 & 81 & MS & 1H-pyridin-4-one & 4-Pyridinol & PYRDINOL & $626-64-2$ & $\mathrm{C} 5 \mathrm{H} 5 \mathrm{NO}$ & Nitrogen \\
\hline 260 & 90.399 & $109 ; 80 ; 53$ & 109 & 76 & MS & 4-aminophenol & 4-Amino-Phenol & AMIPHEOL & $123-30-8$ & $\mathrm{C} 6 \mathrm{H} 7 \mathrm{NO}$ & Phenol \\
\hline
\end{tabular}

${ }^{1}$ RT - Retention Time in minutes.

${ }^{2} \mathrm{TI}$ - Target Ion.

${ }^{3} \mathrm{MP}$ - Match Percent. 
${ }^{4}$ ID - Identification type method used: RS (identified by Reference Standard) and MS (tentatively identified by NIST14 Mass Spectral Library).

Table S3A. Mean and relative standard deviation values of identified VOCs in sugarcane samples from "Amarela", "Radiada" and "Roxa" cultivars.

\begin{tabular}{|c|c|c|c|c|c|c|c|c|c|c|c|c|}
\hline \multirow{4}{*}{ Volatile Organic Compound } & \multicolumn{12}{|c|}{ Relative Peak Areas (x 10 } \\
\hline & \multicolumn{4}{|c|}{ AMA } & \multicolumn{4}{|c|}{ RAD } & \multicolumn{4}{|c|}{ ROX } \\
\hline & \multicolumn{2}{|c|}{2015} & \multicolumn{2}{|c|}{2017} & \multicolumn{2}{|c|}{2015} & \multicolumn{2}{|c|}{2017} & \multicolumn{2}{|c|}{2015} & \multicolumn{2}{|c|}{2017} \\
\hline & Mean & $\begin{array}{c}\text { RSD }^{1} \\
(\%)\end{array}$ & Mean & $\begin{array}{c}\text { RSD }^{1} \\
(\%)\end{array}$ & Mean & $\begin{array}{c}\text { RSD }^{1} \\
(\%)\end{array}$ & Mean & $\begin{array}{c}\text { RSD }^{1} \\
(\%)\end{array}$ & Mean & $\begin{array}{c}\text { RSD }^{1} \\
(\%)\end{array}$ & Mean & $\begin{array}{c}\text { RSD }^{1} \\
(\%)\end{array}$ \\
\hline Pentane & 2.4 & 4.2 & 1.8 & 4.3 & 4.4 & 5.6 & 4.2 & 3.6 & 8.2 & 1.0 & 5.5 & 7.3 \\
\hline 1-Pentene & 1.5 & 7.0 & 1.5 & 2.0 & 1.8 & 9.6 & 1.7 & 7.7 & 2.8 & 1.7 & 2.7 & 4.9 \\
\hline 2-Methyl-Pentane & 0.9 & 1.8 & 1.5 & 3.1 & 1.2 & 7.6 & 2.0 & 5.5 & 4.4 & 3.1 & 7.3 & 8.8 \\
\hline Hexane & 2.1 & 13.0 & 1.5 & 11.0 & 1.2 & 5.0 & 1.3 & 9.1 & 4.0 & 1.3 & 3.7 & 8.5 \\
\hline Ethyl Ether & 5.9 & 9.7 & 0.3 & 7.0 & 3.6 & 4.6 & 0.7 & 3.4 & 0.8 & 3.3 & 1.3 & 9.6 \\
\hline cis-4-Methyl-2-Pentene & 0.4 & 2.4 & 0.6 & 10.4 & 0.2 & 6.1 & 1.2 & 11.1 & 0.9 & 1.6 & 4.2 & 13.1 \\
\hline cis-Piperylene & 3.0 & 17.5 & 2.0 & 5.5 & 5.1 & 8.3 & 6.4 & 1.2 & 10.4 & 2.2 & 20.0 & 9.3 \\
\hline 2-Methyl-1-Pentene & 16.6 & 5.2 & 1.0 & 3.9 & 44.4 & 10.2 & 0.5 & 5.5 & 16.7 & 3.2 & 2.3 & 1.3 \\
\hline cis-3-Methyl-2-Pentene & 1.0 & 0.9 & 1.1 & 5.8 & 7.6 & 5.3 & 4.2 & 2.6 & 1.0 & 3.7 & 1.3 & 7.8 \\
\hline 3-Methyl-Hexane & 0.4 & 6.4 & 0.2 & 12.7 & 0.7 & 5.4 & 0.5 & 2.7 & 0.9 & 1.2 & 0.9 & 9.2 \\
\hline 1,4-Pentadiene & 11.2 & 9.2 & 3.2 & 4.2 & 12.8 & 3.9 & 4.5 & 4.9 & 27.6 & 1.8 & 6.7 & 10.6 \\
\hline Methanethiol & 3.2 & 0.5 & 3.1 & 0.7 & 4.0 & 1.2 & 4.8 & 2.7 & 7.9 & 1.8 & 6.2 & 3.3 \\
\hline trans-Piperylene & 4.0 & 4.3 & 2.3 & 6.8 & 2.6 & 7.5 & 1.5 & 12.8 & 5.6 & 1.9 & 3.0 & 0.3 \\
\hline Heptane & 9.8 & 8.0 & 8.7 & 2.9 & 6.9 & 2.9 & 5.4 & 8.5 & 15.3 & 3.4 & 10.5 & 3.3 \\
\hline tert-Butyl Ethyl Ether & 57.7 & 6.7 & 0.1 & 0.2 & 70.2 & 0.2 & 0.4 & 4.2 & 47.4 & 1.1 & 0.4 & 6.6 \\
\hline Ethanal & 53.9 & 0.0 & 73.5 & 5.9 & 38.7 & 3.1 & 21.9 & 5.0 & 44.9 & 2.8 & 32.1 & 4.6 \\
\hline Dimethyl Sulfide & 785.5 & 2.0 & 807.3 & 3.6 & 583.5 & 2.7 & 417.8 & 4.7 & 323.7 & 1.7 & 294.5 & 7.1 \\
\hline 4-Methyl-Heptane & 20.2 & 10.4 & 7.8 & 3.5 & 12.9 & 3.3 & 4.0 & 0.6 & 17.1 & 3.2 & 6.6 & 3.5 \\
\hline Octane & 5.2 & 8.3 & 4.4 & 9.5 & 7.0 & 3.8 & 6.9 & 4.5 & 9.8 & 6.1 & 8.2 & 4.7 \\
\hline Propanal & 10.6 & 2.2 & 9.1 & 2.2 & 8.1 & 7.6 & 3.6 & 8.4 & 11.3 & 3.2 & 5.8 & 2.0 \\
\hline
\end{tabular}




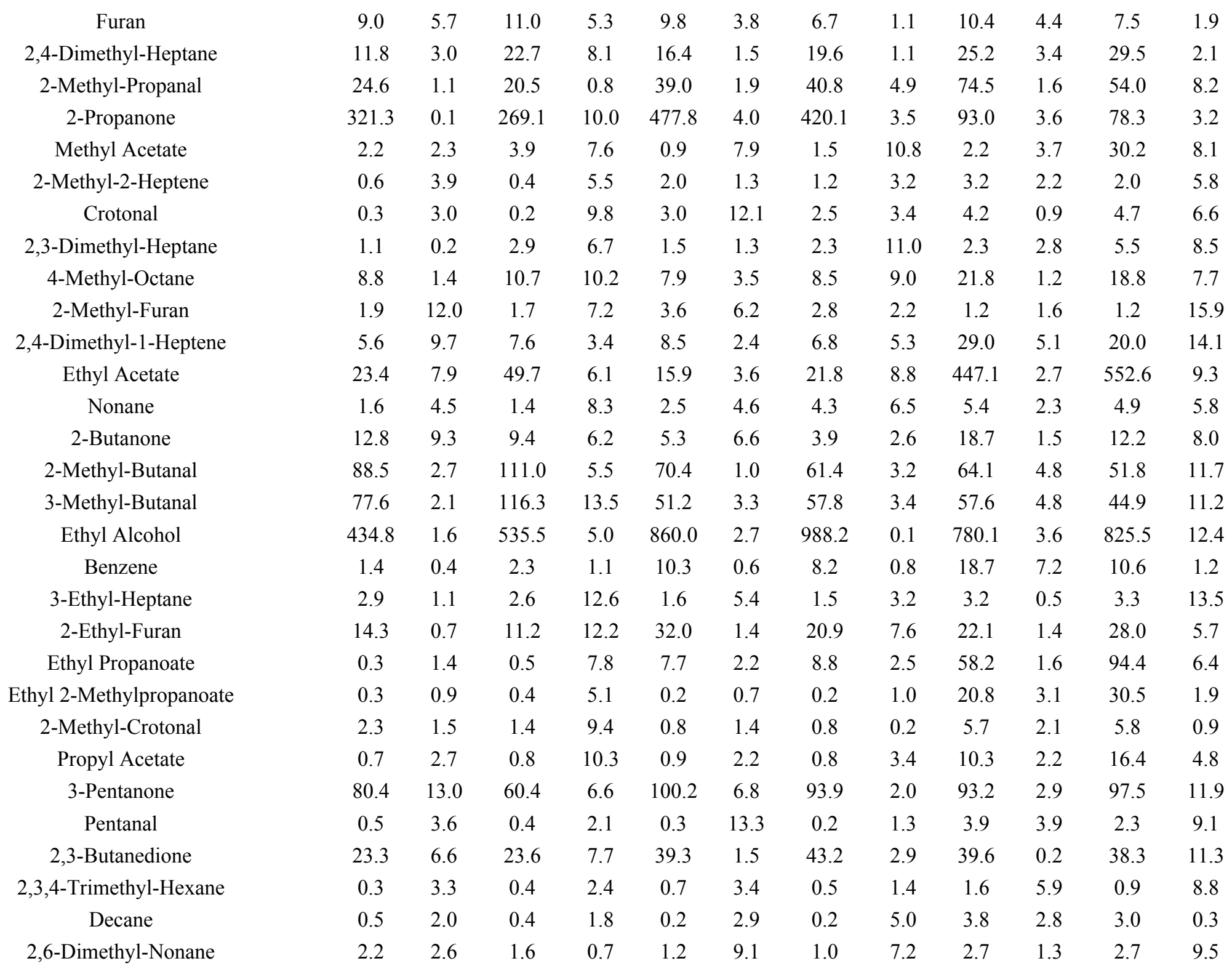




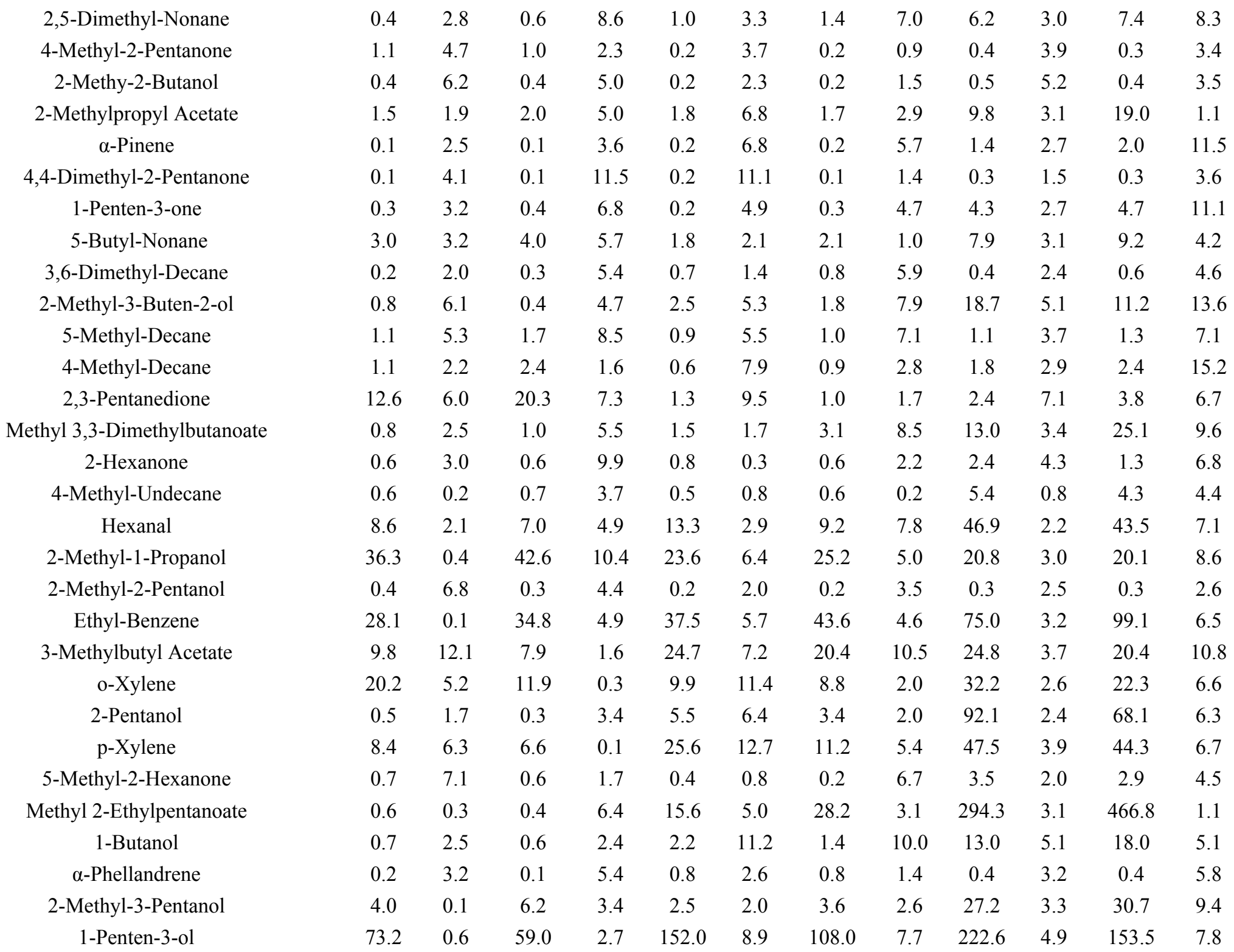




\begin{tabular}{|c|c|c|c|c|c|c|c|c|c|c|c|c|}
\hline$\alpha$-Terpinene & 0.5 & 0.6 & 0.4 & 3.1 & 0.1 & 5.5 & 0.1 & 4.9 & 0.2 & 2.5 & 0.1 & 7.7 \\
\hline Butyl Prop-2-enoate & 4.4 & 0.8 & 6.2 & 6.0 & 4.6 & 6.8 & 1.6 & 10.7 & 10.4 & 3.6 & 19.1 & 4.6 \\
\hline m-Xylene & 1.8 & 2.6 & 1.6 & 7.6 & 15.0 & 9.6 & 15.1 & 4.8 & 40.5 & 2.5 & 31.7 & 4.1 \\
\hline 2-Heptanone & 182.1 & 3.2 & 127.0 & 1.7 & 761.6 & 2.2 & 764.5 & 4.3 & 111.7 & 4.7 & 83.1 & 5.5 \\
\hline Heptanal & 0.5 & 3.5 & 0.4 & 6.7 & 11.1 & 0.6 & 9.3 & 1.7 & 8.2 & 5.6 & 5.0 & 5.2 \\
\hline D-Limonene & 0.1 & 10.9 & 0.2 & 1.5 & 0.2 & 3.7 & 0.2 & 2.6 & 3.1 & 3.7 & 2.7 & 2.8 \\
\hline 3-Hexanol & 0.3 & 4.2 & 0.2 & 10.0 & 1.7 & 0.8 & 2.1 & 4.6 & 0.6 & 2.4 & 0.4 & 7.8 \\
\hline$\beta$-Phellandrene & 0.3 & 2.0 & 0.2 & 2.3 & 1.4 & 1.0 & 1.6 & 1.3 & 0.3 & 1.6 & 0.4 & 4.8 \\
\hline Eucalyptol & 0.2 & 8.2 & 0.2 & 9.4 & 0.1 & 13.5 & 0.2 & 2.7 & 0.2 & 2.4 & 0.1 & 2.2 \\
\hline m-Menthane & 0.1 & 1.1 & 0.1 & 4.2 & 0.2 & 2.0 & 0.2 & 6.0 & 1.6 & 3.1 & 2.6 & 7.5 \\
\hline 4-Methyl-2-Heptanone & 0.3 & 4.1 & 0.3 & 3.0 & 28.4 & 10.7 & 23.6 & 4.1 & 3.9 & 3.9 & 2.2 & 8.1 \\
\hline 3-Methyl-2-Pentanol & 11.3 & 1.0 & 18.4 & 12.6 & 82.1 & 3.3 & 96.5 & 3.5 & 84.7 & 3.0 & 85.7 & 4.3 \\
\hline 2-Methyl-1-Butanol & 160.3 & 5.3 & 231.7 & 8.2 & 193.9 & 3.7 & 189.6 & 5.5 & 136.7 & 4.0 & 231.3 & 13.8 \\
\hline 3-Methyl-1-Butanol & 443.5 & 0.9 & 621.3 & 7.6 & 245.4 & 1.5 & 434.4 & 0.6 & 462.0 & 3.1 & 543.4 & 3.9 \\
\hline o-Ethyltoluene & 1.9 & 3.1 & 1.5 & 13.3 & 5.9 & 0.3 & 3.8 & 1.2 & 8.5 & 0.6 & 8.1 & 4.7 \\
\hline m-Ethyltoluene & 2.1 & 5.0 & 1.0 & 3.0 & 3.6 & 3.9 & 3.9 & 2.0 & 13.5 & 3.7 & 14.8 & 7.3 \\
\hline 2-Hexanol & 59.0 & 2.3 & 78.3 & 6.0 & 49.7 & 3.3 & 63.4 & 10.5 & 3.1 & 1.5 & 1.5 & 13.7 \\
\hline Ethylene Glycol Ethyl Ether & 0.1 & 9.3 & 0.2 & 9.9 & 1.8 & 2.3 & 1.2 & 0.9 & 2.6 & 3.1 & 2.8 & 2.8 \\
\hline 6-Methyl-2-Heptanone & 2.7 & 8.6 & 1.6 & 1.5 & 2.3 & 0.7 & 1.5 & 0.7 & 3.6 & 2.6 & 2.2 & 0.3 \\
\hline Pseudocumene & 2.2 & 2.3 & 3.3 & 1.8 & 5.8 & 3.1 & 4.0 & 5.9 & 7.7 & 3.8 & 7.6 & 5.9 \\
\hline 4,6-Dimethyl-2-Heptanone & 0.7 & 2.8 & 0.9 & 1.0 & 1.0 & 1.9 & 0.7 & 2.4 & 1.6 & 5.1 & 1.1 & 7.3 \\
\hline 2,3-Dimethyl-2-Hexanol & 0.4 & 5.1 & 0.3 & 6.5 & 0.3 & 2.8 & 0.2 & 6.2 & 2.2 & 5.2 & 1.1 & 5.7 \\
\hline 3-Octanone & 6.6 & 3.3 & 5.3 & 2.6 & 22.3 & 4.9 & 16.0 & 3.2 & 23.3 & 2.9 & 18.9 & 8.5 \\
\hline Styrene & 26.5 & 1.3 & 44.1 & 2.8 & 33.5 & 5.8 & 33.6 & 5.8 & 83.5 & 2.8 & 120.2 & 9.9 \\
\hline 5-Methyl-2-Heptanone & 6.4 & 5.3 & 4.3 & 2.2 & 17.7 & 3.9 & 10.0 & 3.4 & 8.8 & 2.7 & 8.7 & 11.0 \\
\hline 1-Pentanol & 31.3 & 4.2 & 48.0 & 8.5 & 27.9 & 1.8 & 32.6 & 5.2 & 4.8 & 2.8 & 4.2 & 5.8 \\
\hline p-Ethyltoluene & 0.2 & 6.5 & 0.4 & 3.8 & 2.3 & 1.9 & 3.1 & 4.0 & 6.8 & 2.2 & 8.3 & 10.4 \\
\hline p-Cymene & 2.1 & 1.4 & 1.4 & 0.2 & 2.2 & 0.4 & 2.5 & 3.3 & 3.7 & 5.4 & 3.8 & 0.0 \\
\hline Mesitylene & 8.4 & 0.8 & 12.6 & 4.7 & 1.2 & 2.7 & 1.3 & 0.1 & 0.4 & 1.7 & 0.5 & 7.8 \\
\hline 4-Methyl-4-Heptanol & 1.8 & 0.2 & 1.1 & 4.9 & 0.6 & 10.2 & 0.5 & 2.6 & 0.2 & 3.3 & 0.1 & 8.8 \\
\hline
\end{tabular}




\begin{tabular}{|c|c|c|c|c|c|c|c|c|c|c|c|c|}
\hline 4-Heptanol & 0.5 & 3.4 & 0.4 & 7.5 & 2.9 & 5.4 & 2.4 & 0.1 & 3.5 & 3.2 & 3.0 & 6.9 \\
\hline 2-Octanone & 36.6 & 7.8 & 23.3 & 6.2 & 115.6 & 1.7 & 99.0 & 0.2 & 20.6 & 2.6 & 13.0 & 9.5 \\
\hline Octanal & 0.2 & 2.2 & 0.2 & 4.3 & 0.1 & 11.3 & 0.2 & 5.8 & 4.4 & 5.4 & 4.1 & 1.7 \\
\hline 3-Hydroxy-2-Butanone & 0.3 & 7.2 & 0.2 & 12.5 & 0.3 & 1.3 & 0.5 & 2.2 & 0.1 & 1.8 & 0.1 & 12.2 \\
\hline 1-Octen-3-one & 15.6 & 3.0 & 22.1 & 6.8 & 7.4 & 2.6 & 6.4 & 3.0 & 3.2 & 3.8 & 2.9 & 10.1 \\
\hline trans-2-Pentenol & 12.8 & 5.2 & 10.8 & 3.5 & 22.6 & 4.0 & 23.7 & 1.4 & 23.4 & 3.4 & 24.4 & 7.6 \\
\hline 2,2,6-Trimethyl-Cyclohexanone & 2.5 & 4.9 & 2.2 & 6.6 & 3.2 & 7.0 & 3.0 & 2.8 & 4.8 & 2.9 & 5.4 & 4.3 \\
\hline 2-Heptanol & 531.4 & 5.3 & 575.8 & 2.4 & 530.4 & 8.7 & 567.5 & 11.3 & 270.3 & 3.6 & 276.8 & 4.7 \\
\hline cis-2-Pentenol & 214.3 & 3.5 & 301.2 & 10.2 & 138.5 & 13.4 & 191.0 & 5.6 & 147.6 & 2.8 & 148.4 & 5.0 \\
\hline 1-Hydroxy-2-Propanone & 20.3 & 0.3 & 23.6 & 4.6 & 9.3 & 3.5 & 11.0 & 7.9 & 5.4 & 2.3 & 6.9 & 3.2 \\
\hline 5-Methyl-4-Hexen-3-one & 1.5 & 9.6 & 1.2 & 0.2 & 3.2 & 8.9 & 2.2 & 8.2 & 2.0 & 2.7 & 1.5 & 12.8 \\
\hline Hemimellitene & 4.9 & 8.6 & 4.1 & 4.2 & 3.5 & 7.2 & 2.3 & 3.7 & 9.2 & 3.3 & 7.7 & 9.6 \\
\hline 6-Methyl-5-Hepten-2-one & 14.7 & 4.2 & 20.0 & 8.2 & 9.4 & 3.5 & 13.2 & 0.8 & 17.2 & 0.3 & 25.0 & 2.7 \\
\hline 2-Pentene & 287.3 & 5.2 & 325.7 & 11.6 & 456.2 & 0.7 & 422.4 & 5.1 & 143.1 & 3.6 & 96.5 & 3.8 \\
\hline 1-Hexanol & 542.7 & 2.8 & 677.6 & 6.4 & 392.5 & 6.7 & 413.2 & 2.8 & 701.8 & 2.2 & 658.5 & 4.3 \\
\hline 2-Cyclopenten-1-one & 1.2 & 7.4 & 1.0 & 12.3 & 11.4 & 10.5 & 9.7 & 0.8 & 14.8 & 6.5 & 8.1 & 5.0 \\
\hline 2-Methyl-2-Cyclopenten-1-one & 0.2 & 0.6 & 0.3 & 8.1 & 3.7 & 4.5 & 3.1 & 5.5 & 3.2 & 5.1 & 2.9 & 1.6 \\
\hline$\beta$-Methy-Styrene & 0.3 & 6.0 & 0.2 & 5.3 & 1.7 & 3.1 & 2.0 & 2.5 & 4.1 & 1.3 & 3.4 & 10.6 \\
\hline 4-Octanol & 3.7 & 5.7 & 3.5 & 5.1 & 4.0 & 1.1 & 3.5 & 5.3 & 2.3 & 5.8 & 1.7 & 3.0 \\
\hline cis-3-Hexen-1-ol & 15.0 & 10.4 & 11.2 & 12.1 & 6.8 & 0.7 & 8.7 & 2.6 & 10.7 & 3.4 & 9.7 & 12.6 \\
\hline 2-Nonanone & 57.2 & 4.3 & 48.5 & 4.1 & 370.9 & 4.0 & 330.3 & 10.2 & 60.4 & 1.5 & 60.9 & 14.0 \\
\hline Nonanal & 82.7 & 2.3 & 88.3 & 5.7 & 78.2 & 1.2 & 87.7 & 0.3 & 24.7 & 6.2 & 13.5 & 11.7 \\
\hline Ethylene Glycol Butyl Ether & 27.8 & 3.1 & 27.2 & 5.7 & 2.9 & 1.0 & 2.5 & 4.1 & 10.3 & 3.2 & 15.2 & 13.6 \\
\hline 1-Ethyl-1-Methyl-Cyclopentane & 2.2 & 2.8 & 1.6 & 3.0 & 3.0 & 4.1 & 3.9 & 2.2 & 17.6 & 4.8 & 12.3 & 10.2 \\
\hline 3-Octen-2-one & 2.8 & 8.8 & 2.9 & 12.3 & 85.0 & 3.3 & 164.4 & 4.5 & 7.4 & 4.7 & 6.9 & 4.8 \\
\hline 2-Octanol & 78.0 & 0.2 & 99.2 & 10.9 & 158.9 & 10.5 & 166.3 & 4.8 & 10.4 & 0.9 & 10.7 & 1.9 \\
\hline 3-Ethyl-2-Methyl-1,3-Hexadiene & 0.3 & 4.3 & 0.3 & 8.4 & 0.3 & 6.8 & 0.3 & 2.6 & 0.9 & 1.0 & 1.0 & 10.7 \\
\hline 1,3-Bis(1,1-Dimethylethyl)-Benzene & 0.4 & 1.3 & 0.3 & 6.0 & 0.2 & 2.0 & 0.2 & 0.6 & 0.2 & 6.2 & 0.2 & 14.2 \\
\hline trans-2-Octenal & 0.9 & 2.0 & 0.8 & 4.4 & 8.5 & 3.3 & 9.9 & 0.3 & 22.2 & 5.4 & 17.9 & 4.8 \\
\hline m-Ethyl-Styrene & 9.7 & 5.4 & 10.7 & 6.2 & 8.5 & 2.8 & 9.9 & 4.7 & 2.1 & 1.9 & 1.1 & 6.1 \\
\hline
\end{tabular}




\begin{tabular}{|c|c|c|c|c|c|c|c|c|c|c|c|c|}
\hline 5-Methyl-2-Heptanone & 0.7 & 3.9 & 0.6 & 7.1 & 0.6 & 7.4 & 0.5 & 3.6 & 0.9 & 3.4 & 0.8 & 6.8 \\
\hline 2-Decanone & 2.4 & 4.0 & 1.8 & 6.7 & 2.7 & 3.8 & 2.0 & 12.8 & 1.7 & 3.7 & 1.2 & 13.3 \\
\hline 1-Octen-3-ol & 135.1 & 10.2 & 116.5 & 8.5 & 135.5 & 0.0 & 105.3 & 6.8 & 145.0 & 6.1 & 122.2 & 5.4 \\
\hline trans,trans-2,4-Heptadienal & 1.9 & 4.1 & 2.2 & 4.4 & 4.3 & 2.5 & 4.6 & 5.5 & 2.6 & 4.4 & 2.9 & 9.6 \\
\hline 1-Heptanol & 52.4 & 0.9 & 53.5 & 10.1 & 55.3 & 0.8 & 49.9 & 0.2 & 52.6 & 3.0 & 59.4 & 11.7 \\
\hline cis,cis-3,5-Octadiene & 2.3 & 13.4 & 1.7 & 8.6 & 2.2 & 4.1 & 2.1 & 1.4 & 1.0 & 6.3 & 1.4 & 7.9 \\
\hline Methyl 2-Oxopropanoate & 142.4 & 8.5 & 171.8 & 9.2 & 91.1 & 0.3 & 116.6 & 4.6 & 15.1 & 1.9 & 15.9 & 8.9 \\
\hline Dihydromyrcenol & 15.3 & 2.4 & 20.0 & 12.9 & 11.8 & 6.8 & 13.1 & 5.5 & 9.4 & 3.7 & 11.8 & 5.0 \\
\hline trans-Menthone & 0.1 & 9.8 & 0.1 & 3.6 & 0.9 & 8.3 & 1.1 & 10.5 & 0.4 & 4.7 & 0.4 & 4.8 \\
\hline Furfural & 62.1 & 4.4 & 79.3 & 3.4 & 64.9 & 7.1 & 88.0 & 2.2 & 75.8 & 3.2 & 54.7 & 3.3 \\
\hline Ethanoic Acid & 1884.8 & 1.3 & 2262.6 & 13.3 & 1967.9 & 8.6 & 2388.6 & 1.7 & 1360.3 & 3.2 & 1538.3 & 4.4 \\
\hline 2-Ethyl-1-Hexanol & 31.9 & 4.3 & 34.2 & 12.1 & 13.8 & 6.6 & 13.2 & 2.8 & 31.9 & 5.0 & 29.7 & 4.1 \\
\hline Decanal & 25.2 & 3.8 & 33.3 & 1.8 & 43.5 & 9.1 & 52.0 & 1.5 & 63.0 & 4.9 & 65.2 & 4.8 \\
\hline Furfuryl Formate & 49.0 & 4.5 & 55.5 & 5.4 & 46.3 & 3.5 & 56.3 & 2.6 & 32.0 & 1.8 & 32.1 & 2.0 \\
\hline trans-2-Hepten-1-ol & 22.3 & 7.4 & 22.1 & 11.9 & 20.9 & 12.0 & 19.5 & 11.7 & 43.3 & 4.0 & 42.5 & 10.1 \\
\hline 2-Nonanol & 20.7 & 5.5 & 24.4 & 3.5 & 378.8 & 1.5 & 344.5 & 4.7 & 4.1 & 2.6 & 3.8 & 1.7 \\
\hline 1-(2-Furanyl)-Ethanone & 306.4 & 2.2 & 365.3 & 9.5 & 492.2 & 1.4 & 456.3 & 5.2 & 60.9 & 2.1 & 69.3 & 6.7 \\
\hline 3,5-Octadien-2-one & 10.9 & 5.0 & 12.5 & 7.1 & 6.8 & 3.1 & 7.2 & 5.9 & 25.6 & 5.2 & 21.7 & 2.3 \\
\hline Benzaldehyde & 29.1 & 2.2 & 31.9 & 10.8 & 27.7 & 1.4 & 23.3 & 8.5 & 42.9 & 3.2 & 33.4 & 0.4 \\
\hline Furfuryl Acetate & 0.4 & 3.4 & 0.4 & 6.6 & 0.4 & 2.2 & 0.4 & 6.5 & 0.9 & 4.4 & 0.8 & 7.9 \\
\hline Linalool & 6.6 & 3.3 & 7.4 & 8.0 & 5.1 & 8.3 & 4.9 & 2.3 & 6.2 & 5.4 & 6.1 & 4.1 \\
\hline$\beta$-Myrcene & 0.8 & 6.0 & 1.0 & 3.2 & 1.2 & 13.3 & 1.0 & 9.4 & 1.5 & 3.6 & 1.1 & 3.9 \\
\hline 1-Octanol & 83.0 & 3.1 & 93.3 & 8.3 & 73.8 & 5.3 & 65.8 & 1.5 & 124.7 & 2.9 & 123.7 & 11.4 \\
\hline trans-p-2-Menthen-1-ol & 0.1 & 5.4 & 0.1 & 2.2 & 0.7 & 2.9 & 0.9 & 7.2 & 0.6 & 3.4 & 0.6 & 7.3 \\
\hline Propanoic Acid & 4.2 & 5.5 & 4.6 & 7.7 & 1.0 & 0.1 & 0.9 & 2.4 & 1.5 & 4.4 & 1.5 & 6.7 \\
\hline 2,3-Butanediol & 231.1 & 0.3 & 338.0 & 7.0 & 431.1 & 3.7 & 451.8 & 1.8 & 57.7 & 3.0 & 57.3 & 10.0 \\
\hline Dimethyl Sulfoxide & 62.1 & 4.9 & 68.7 & 7.3 & 32.0 & 5.4 & 35.2 & 2.8 & 19.5 & 3.5 & 25.3 & 4.3 \\
\hline m-Menth-3-ene & 49.7 & 2.0 & 58.2 & 6.5 & 44.1 & 1.0 & 55.2 & 4.0 & 6.5 & 4.3 & 7.8 & 6.4 \\
\hline Trans-(2-Ethylcyclopentyl)-Methanol & 20.6 & 3.1 & 28.6 & 4.7 & 33.6 & 1.7 & 35.7 & 4.4 & 33.6 & 3.0 & 39.8 & 13.9 \\
\hline Bornyl Acetate & 9.1 & 1.2 & 10.6 & 6.1 & 4.8 & 0.7 & 5.4 & 1.8 & 9.4 & 3.6 & 10.2 & 14.2 \\
\hline
\end{tabular}




\begin{tabular}{|c|c|c|c|c|c|c|c|c|c|c|c|c|}
\hline 4-Cyclopentene-1,3-dione & 271.0 & 1.4 & 308.9 & 6.3 & 268.6 & 3.0 & 269.8 & 1.0 & 142.0 & 2.2 & 97.1 & 2.7 \\
\hline Menthol & 2.8 & 5.5 & 3.6 & 1.8 & 10.5 & 3.3 & 10.2 & 11.3 & 0.5 & 5.3 & 0.3 & 10.8 \\
\hline 2-Undecanone & 9.4 & 2.4 & 11.1 & 10.4 & 24.6 & 4.4 & 32.2 & 10.7 & 7.0 & 1.7 & 7.3 & 4.1 \\
\hline 2-(2-Furanyl)-Furan & 2.4 & 4.0 & 3.5 & 6.3 & 3.0 & 1.9 & 3.6 & 1.7 & 3.8 & 3.9 & 4.0 & 10.2 \\
\hline trans-2-Octen-1-ol & 38.0 & 9.9 & 45.9 & 10.4 & 53.6 & 1.6 & 47.6 & 8.4 & 101.3 & 3.6 & 99.3 & 2.0 \\
\hline trans-2-Nonen-1-ol & 24.0 & 8.8 & 22.4 & 6.2 & 14.5 & 3.3 & 14.9 & 4.3 & 34.4 & 2.4 & 30.5 & 5.2 \\
\hline Diethylene Glycol Ethyl Ether & 63.6 & 3.7 & 70.0 & 12.0 & 53.0 & 1.3 & 45.0 & 3.5 & 71.2 & 4.0 & 65.3 & 2.2 \\
\hline 5,5-Trimethyl-2-Cyclopenten-1-one & 12.6 & 10.7 & 17.5 & 9.3 & 13.9 & 6.5 & 10.9 & 1.7 & 20.5 & 3.2 & 12.3 & 9.7 \\
\hline cis-p-2-Menthen-1-ol & 1.9 & 3.5 & 1.5 & 3.5 & 3.8 & 2.3 & 3.3 & 3.1 & 2.4 & 4.8 & 2.8 & 9.9 \\
\hline$\beta$-Cyclocitral & 3.2 & 5.8 & 3.1 & 5.9 & 4.7 & 2.8 & 4.4 & 0.9 & 10.5 & 4.2 & 10.0 & 5.8 \\
\hline Benzeneacetaldehyde & 91.3 & 5.6 & 94.5 & 6.5 & 35.0 & 0.7 & 38.2 & 3.0 & 18.8 & 3.9 & 18.2 & 7.1 \\
\hline 1-Nonanol & 25.0 & 4.2 & 31.2 & 12.8 & 36.8 & 1.0 & 43.0 & 1.5 & 30.4 & 4.4 & 32.7 & 4.3 \\
\hline Furfuryl Alcohol & 60.2 & 2.2 & 58.2 & 3.8 & 78.2 & 11.4 & 89.0 & 0.3 & 72.5 & 1.0 & 81.3 & 3.9 \\
\hline Phoracanthol & 66.3 & 0.7 & 76.6 & 6.8 & 12.4 & 3.3 & 17.0 & 3.2 & 10.4 & 2.8 & 10.2 & 12.0 \\
\hline cis-3-Nonen-1-ol & 18.0 & 2.4 & 21.1 & 9.9 & 8.2 & 5.5 & 9.6 & 3.7 & 10.2 & 1.4 & 11.8 & 6.1 \\
\hline cis-Piperitol & 3.7 & 1.2 & 3.0 & 7.5 & 1.7 & 7.9 & 2.3 & 7.5 & 0.6 & 2.8 & 0.6 & 7.6 \\
\hline p-Methoxy-Styrene & 5.3 & 0.3 & 6.7 & 10.5 & 1.6 & 2.7 & 1.6 & 3.6 & 23.4 & 1.8 & 27.8 & 8.6 \\
\hline (2Z)-3-Ethyl-2,4-Pentadien-1-ol & 9.3 & 2.9 & 10.5 & 9.9 & 7.0 & 0.5 & 6.8 & 2.7 & 6.6 & 0.8 & 6.7 & 2.6 \\
\hline Butylene Glycol Monobutyl Ether & 13.7 & 4.8 & 17.5 & 7.3 & 12.0 & 6.1 & 16.7 & 5.8 & 8.8 & 1.3 & 9.4 & 7.7 \\
\hline$\alpha$-Terpineol & 2.4 & 3.5 & 2.0 & 4.7 & 2.3 & 1.8 & 2.2 & 1.2 & 1.0 & 0.2 & 1.1 & 14.0 \\
\hline 1,5-Cyclooctanedione & 2.1 & 3.3 & 2.0 & 1.6 & 4.2 & 0.5 & 0.5 & 2.3 & 2.0 & 5.2 & 2.1 & 4.2 \\
\hline$\gamma$-Terpinene & 1.8 & 1.9 & 2.0 & 4.9 & 3.2 & 0.2 & 4.0 & 12.4 & 6.3 & 2.4 & 6.6 & 12.9 \\
\hline trans-2-Decenol & 6.2 & 1.3 & 7.2 & 8.1 & 7.0 & 6.7 & 7.4 & 2.6 & 12.4 & 5.3 & 10.1 & 6.7 \\
\hline 2-Propenoic Acid & 8.8 & 1.3 & 12.9 & 11.3 & 1.0 & 5.3 & 1.0 & 2.8 & 0.5 & 4.9 & 0.5 & 4.7 \\
\hline 2-Undecanol & 4.4 & 8.3 & 4.6 & 6.4 & 83.2 & 4.6 & 76.2 & 0.1 & 3.5 & 4.6 & 2.3 & 8.7 \\
\hline 3-Ethyl-Benzaldehyde & 6.1 & 8.8 & 5.3 & 4.4 & 4.9 & 3.8 & 4.3 & 3.6 & 6.3 & 4.3 & 6.0 & 5.2 \\
\hline 3-Hydroxy-Benzaldehyde & 13.0 & 0.1 & 19.5 & 13.2 & 1.9 & 8.2 & 1.7 & 4.6 & 4.5 & 3.3 & 4.8 & 8.8 \\
\hline trans-2-Nonene & 3.4 & 1.6 & 3.4 & 6.9 & 4.7 & 6.1 & 4.8 & 12.3 & 0.5 & 3.2 & 0.4 & 6.3 \\
\hline 2-Methyl-2-Pentenal & 14.5 & 3.2 & 17.0 & 5.6 & 7.3 & 7.7 & 7.4 & 1.5 & 6.8 & 4.2 & 6.6 & 2.2 \\
\hline cis-5-Decenol & 5.2 & 2.5 & 5.3 & 5.0 & 105.3 & 8.8 & 110.7 & 10.1 & 2.7 & 6.0 & 1.7 & 13.3 \\
\hline
\end{tabular}




\begin{tabular}{|c|c|c|c|c|c|c|c|c|c|c|c|c|}
\hline trans-Piperitol & 2.3 & 12.0 & 2.3 & 7.8 & 4.6 & 6.1 & 5.5 & 2.8 & 0.9 & 4.3 & 0.9 & 11.8 \\
\hline 4-Ethyl-Benzaldehyde & 3.4 & 2.3 & 3.7 & 8.7 & 3.0 & 10.5 & 2.9 & 1.3 & 5.5 & 5.0 & 5.2 & 6.0 \\
\hline Naphthalene & 16.4 & 0.4 & 17.0 & 12.4 & 7.5 & 0.7 & 8.3 & 0.9 & 19.1 & 3.5 & 20.4 & 8.2 \\
\hline 1-Decanol & 31.0 & 1.4 & 36.2 & 10.4 & 17.0 & 2.0 & 19.3 & 5.7 & 19.7 & 6.1 & 16.9 & 7.8 \\
\hline Cyclodecane & 108.9 & 10.0 & 134.2 & 12.0 & 89.5 & 5.4 & 87.3 & 2.3 & 67.0 & 2.6 & 65.2 & 4.0 \\
\hline trans,trans-2,4-Decadienal & 12.0 & 2.2 & 18.5 & 6.5 & 19.4 & 0.5 & 23.4 & 8.4 & 49.8 & 1.2 & 54.6 & 4.7 \\
\hline 1,2-Cyclopentanedione & 545.7 & 2.7 & 603.5 & 9.8 & 425.5 & 2.0 & 454.4 & 0.6 & 186.6 & 2.4 & 201.0 & 3.4 \\
\hline Cyclodecene & 2.2 & 0.5 & 2.3 & 13.5 & 2.0 & 2.5 & 2.1 & 6.1 & 1.9 & 4.3 & 1.5 & 9.7 \\
\hline cis-4-Decenol & 1.8 & 2.6 & 1.7 & 12.2 & 3.0 & 0.1 & 3.1 & 7.5 & 0.6 & 3.8 & 0.6 & 5.2 \\
\hline 1-Phenyl-2-Butanone & 7.4 & 1.1 & 8.5 & 5.9 & 7.5 & 2.7 & 7.2 & 5.6 & 15.2 & 5.6 & 15.3 & 3.1 \\
\hline Phenethyl Alcohol & 12.6 & 6.6 & 15.0 & 9.0 & 2.2 & 1.3 & 2.3 & 8.4 & 5.7 & 1.4 & 5.3 & 2.9 \\
\hline trans,cis-2,4-Decadienal & 8.2 & 2.9 & 9.6 & 5.3 & 12.9 & 2.5 & 15.2 & 4.8 & 42.7 & 5.1 & 44.1 & 8.5 \\
\hline 2-Cyclohexenol & 76.5 & 3.8 & 82.0 & 5.0 & 37.0 & 0.2 & 47.4 & 1.1 & 4.6 & 3.5 & 4.5 & 10.9 \\
\hline 2-Ethyl Hexanoic Acid & 20.5 & 1.3 & 28.1 & 4.0 & 4.1 & 1.4 & 4.4 & 4.6 & 49.9 & 2.7 & 55.2 & 1.9 \\
\hline 3-Methyl-1,2-Cyclopentanedione & 27.0 & 2.7 & 34.6 & 3.8 & 46.7 & 1.8 & 52.8 & 2.2 & 8.6 & 0.0 & 9.2 & 7.7 \\
\hline trans-Cinnamaldehyde & 9.2 & 0.8 & 11.6 & 4.1 & 5.8 & 0.6 & 5.7 & 3.5 & 7.8 & 2.0 & 8.6 & 3.7 \\
\hline Heptanoic Acid & 82.6 & 4.7 & 77.6 & 6.2 & 37.0 & 1.6 & 30.4 & 2.0 & 86.7 & 0.4 & 80.7 & 10.7 \\
\hline Mequinol & 28.6 & 6.8 & 27.4 & 0.1 & 19.9 & 1.2 & 24.6 & 3.1 & 81.3 & 3.5 & 84.2 & 0.3 \\
\hline 2,4,5-Trihydroxypyrimidine & 67.0 & 5.1 & 71.0 & 1.7 & 11.1 & 11.4 & 16.0 & 6.5 & 10.4 & 3.3 & 10.9 & 7.6 \\
\hline cis-Cinnamaldehyde & 8.8 & 7.4 & 9.3 & 5.2 & 8.6 & 7.0 & 8.9 & 1.2 & 7.6 & 4.0 & 8.0 & 6.4 \\
\hline Benzenemethanol & 46.5 & 0.7 & 50.2 & 10.1 & 22.5 & 8.9 & 26.9 & 5.3 & 106.3 & 3.4 & 141.1 & 6.8 \\
\hline Mesitaldehyde & 5.0 & 1.4 & 6.0 & 6.3 & 2.1 & 0.7 & 2.9 & 2.7 & 6.0 & 4.3 & 6.5 & 8.7 \\
\hline 2-Ethylhexyl Octanoate & 1.1 & 8.7 & 1.4 & 10.3 & 3.0 & 4.6 & 2.9 & 2.4 & 0.8 & 5.4 & 0.8 & 6.8 \\
\hline Benzeneethanol & 95.5 & 6.7 & 117.1 & 8.4 & 28.9 & 0.0 & 32.9 & 6.4 & 47.9 & 3.8 & 47.8 & 11.8 \\
\hline 1,4-Butanediol & 83.2 & 1.1 & 93.9 & 3.9 & 65.0 & 0.2 & 72.7 & 6.6 & 62.7 & 5.1 & 60.3 & 1.5 \\
\hline$\beta$-Ionone & 13.3 & 6.6 & 15.2 & 10.0 & 11.8 & 3.4 & 11.6 & 3.0 & 44.1 & 5.7 & 36.8 & 3.6 \\
\hline 2-Methyl-1,4-Benzenediol & 21.1 & 4.2 & 26.7 & 6.8 & 12.9 & 0.7 & 19.0 & 1.0 & 4.0 & 0.8 & 3.4 & 6.1 \\
\hline 2-Ethyl Heptanoic Acid & 1.0 & 4.5 & 1.1 & 11.5 & 5.5 & 4.8 & 5.2 & 0.9 & 1.6 & 0.3 & 1.6 & 5.0 \\
\hline 1-Dodecanol & 12.8 & 8.9 & 10.5 & 10.2 & 5.4 & 7.6 & 6.5 & 7.2 & 15.2 & 1.9 & 10.7 & 2.5 \\
\hline Benzalacetone & 18.3 & 0.7 & 18.7 & 1.8 & 9.3 & 3.4 & 8.6 & 0.9 & 10.2 & 4.5 & 10.8 & 13.1 \\
\hline
\end{tabular}




\begin{tabular}{|c|c|c|c|c|c|c|c|c|c|c|c|c|}
\hline Maltol & 97.5 & 5.2 & 151.4 & 4.2 & 128.6 & 1.1 & 173.1 & 3.5 & 65.5 & 3.9 & 66.3 & 5.4 \\
\hline 2-Acetylpyrrole & 18.5 & 1.7 & 18.6 & 4.6 & 20.9 & 4.0 & 19.9 & 1.1 & 0.6 & 4.8 & 0.6 & 3.5 \\
\hline 2,4-Decadien-1-ol & 8.2 & 2.0 & 10.9 & 10.8 & 7.8 & 0.6 & 8.1 & 1.6 & 7.7 & 3.4 & 8.4 & 3.6 \\
\hline Levoglucosenone & 9.0 & 2.2 & 10.5 & 11.1 & 16.4 & 4.3 & 12.8 & 2.5 & 9.0 & 3.5 & 8.3 & 5.5 \\
\hline Phenol & 36.8 & 12.1 & 46.0 & 11.0 & 39.2 & 7.3 & 46.6 & 1.1 & 63.1 & 6.3 & 51.9 & 6.0 \\
\hline 1-(2-Furanyl)-2-Hydroxy-Ethanone & 48.4 & 4.9 & 45.7 & 10.7 & 28.6 & 0.4 & 22.7 & 1.1 & 5.2 & 3.6 & 4.5 & 8.1 \\
\hline 4,6-Dimethyl-Dodecane & 20.2 & 6.2 & 25.7 & 13.3 & 30.9 & 1.4 & 31.4 & 3.5 & 3.5 & 2.6 & 3.6 & 9.7 \\
\hline Ethyl Decanoate & 5.8 & 0.6 & 6.1 & 12.3 & 5.2 & 2.5 & 5.8 & 3.1 & 5.1 & 3.7 & 5.3 & 4.3 \\
\hline 1-Methyl-4-Piperidinone & 1.6 & 12.8 & 1.4 & 12.1 & 0.5 & 2.8 & 0.6 & 2.8 & 0.4 & 3.4 & 0.4 & 8.4 \\
\hline 2,2-Dimethyl-3-Heptanone & 2.7 & 1.6 & 2.9 & 12.2 & 102.8 & 2.5 & 112.3 & 0.2 & 4.1 & 1.9 & 3.9 & 5.5 \\
\hline Octanoic Acid & 30.2 & 1.4 & 39.4 & 9.5 & 1.3 & 4.6 & 1.5 & 11.2 & 18.6 & 5.1 & 17.6 & 12.3 \\
\hline 1,3-Dihydroxy-2-Propanone & 82.2 & 5.1 & 92.9 & 10.2 & 43.3 & 0.2 & 38.9 & 6.5 & 2.0 & 3.9 & 1.3 & 12.3 \\
\hline 2-Phenoxy-Ethanol & 6.9 & 1.1 & 7.8 & 5.9 & 6.2 & 4.3 & 5.5 & 3.6 & 17.8 & 1.6 & 15.3 & 11.2 \\
\hline Isophthalaldehyde & 5.0 & 6.8 & 4.4 & 8.6 & 4.8 & 4.0 & 4.3 & 1.5 & 5.4 & 3.2 & 4.8 & 5.3 \\
\hline Cyclotetradecane & 8.6 & 0.5 & 9.4 & 9.5 & 2.2 & 1.8 & 2.8 & 4.7 & 4.9 & 1.8 & 4.9 & 4.9 \\
\hline Nonanoic Acid & 15.5 & 8.2 & 19.6 & 10.6 & 1.7 & 1.7 & 1.4 & 3.8 & 47.2 & 4.3 & 42.5 & 5.8 \\
\hline 2-Ethyl-Phenol & 15.5 & 7.1 & 19.9 & 7.1 & 12.2 & 1.9 & 15.3 & 4.3 & 26.5 & 1.9 & 26.6 & 6.8 \\
\hline 2-Methoxy-4-Vinyl-Phenol & 61.9 & 1.6 & 64.8 & 4.0 & 53.3 & 0.2 & 55.3 & 8.0 & 32.3 & 0.2 & 29.1 & 8.7 \\
\hline 4-Methylimidazole & 1.2 & 9.1 & 1.0 & 4.5 & 18.9 & 5.7 & 22.8 & 6.1 & 24.2 & 1.1 & 29.0 & 12.6 \\
\hline m-tert-Butyl-Toluene & 15.6 & 4.1 & 17.7 & 6.7 & 16.9 & 2.0 & 17.3 & 6.5 & 18.8 & 4.5 & 17.7 & 6.5 \\
\hline Syringol & 22.4 & 10.2 & 20.4 & 7.8 & 17.8 & 0.8 & 19.4 & 0.7 & 13.7 & 1.2 & 13.1 & 6.3 \\
\hline Decanoic Acid & 33.7 & 1.2 & 31.6 & 11.5 & 6.1 & 7.5 & 5.2 & 1.0 & 32.8 & 4.9 & 29.8 & 9.3 \\
\hline 3-Hydroxy-2,3-Dihydro-Maltol & 58.3 & 1.2 & 64.1 & 3.3 & 18.5 & 3.5 & 19.0 & 1.2 & 42.3 & 3.6 & 37.6 & 11.8 \\
\hline 5-Hydroxy-Maltol & 36.2 & 5.2 & 37.3 & 5.6 & 71.6 & 1.5 & 68.6 & 2.4 & 41.6 & 5.6 & 30.5 & 9.8 \\
\hline Triethylene Glycol & 1.2 & 4.6 & 1.0 & 13.4 & 1.3 & 7.2 & 1.0 & 8.4 & 5.5 & 2.9 & 5.8 & 4.9 \\
\hline Benzoic Acid & 13.5 & 7.9 & 19.8 & 3.1 & 6.0 & 0.9 & 6.7 & 1.0 & 5.4 & 4.9 & 5.5 & 5.4 \\
\hline 2-Methyl-Benzaldehyde & 193.4 & 1.3 & 209.8 & 6.9 & 132.7 & 6.1 & 184.7 & 4.2 & 255.2 & 5.1 & 230.2 & 6.2 \\
\hline Undecanoic Acid & 32.5 & 5.8 & 31.7 & 10.7 & 4.7 & 0.0 & 5.6 & 3.3 & 30.9 & 1.0 & 29.9 & 5.9 \\
\hline 4-Pyridinol & 92.0 & 0.4 & 117.0 & 13.7 & 5.4 & 1.8 & 4.7 & 5.9 & 46.0 & 1.0 & 39.9 & 7.3 \\
\hline 4-Amino-Phenol & 49.8 & 1.2 & 51.9 & 6.9 & 6.0 & 5.7 & 5.9 & 1.1 & 2.0 & 3.3 & 1.7 & 11.9 \\
\hline
\end{tabular}


${ }^{1}$ RSD - Relative Standard Deviation values.

Table S3B. Mean and relative standard deviation values of identified VOCs in sugarcane samples from "Verde", "Violeta" and "Canica" cultivars.

\begin{tabular}{|c|c|c|c|c|c|c|c|c|c|c|c|c|}
\hline \multirow{3}{*}{ Volatile Organic Compound } & \multicolumn{4}{|c|}{ VER } & \multicolumn{4}{|c|}{ VIO } & \multicolumn{4}{|c|}{ CAN } \\
\hline & \multicolumn{2}{|c|}{2015} & \multicolumn{2}{|c|}{2017} & \multicolumn{2}{|c|}{2015} & \multicolumn{2}{|c|}{2017} & \multicolumn{2}{|c|}{2015} & \multicolumn{2}{|c|}{2017} \\
\hline & Mean & $\begin{array}{l}\text { RSD }^{1} \\
(\%)\end{array}$ & Mean & $\begin{array}{c}\text { RSD }^{1} \\
(\%)\end{array}$ & Mean & $\begin{array}{c}\text { RSD }^{1} \\
(\%)\end{array}$ & Mean & $\begin{array}{c}\text { RSD }^{1} \\
\text { (\%) }\end{array}$ & Mean & $\begin{array}{l}\text { RSD }^{1} \\
(\%)\end{array}$ & Mean & $\operatorname{RSD}^{\text {RS) }^{1}}$ \\
\hline Pentane & 2.6 & 4.2 & 2.1 & 8.9 & 3.0 & 1.1 & 3.6 & 5.3 & 2.2 & 0.2 & 2.1 & 7.3 \\
\hline 1-Pentene & 1.1 & 7.3 & 1.5 & 0.8 & 0.8 & 1.5 & 1.0 & 5.9 & 2.0 & 0.8 & 3.4 & 3.3 \\
\hline 2-Methyl-Pentane & 2.8 & 3.3 & 3.7 & 5.5 & 1.0 & 3.6 & 1.2 & 5.3 & 0.1 & 0.5 & 0.2 & 9.8 \\
\hline Hexane & 2.2 & 3.7 & 2.2 & 11.2 & 1.6 & 7.7 & 1.6 & 8.1 & 20.4 & 3.6 & 10.6 & 1.2 \\
\hline Ethyl Ether & 0.3 & 2.9 & 0.2 & 6.4 & 1.4 & 4.0 & 1.0 & 4.0 & 5.3 & 7.3 & 2.5 & 11.0 \\
\hline cis-4-Methyl-2-Pentene & 0.1 & 0.6 & 2.5 & 2.9 & 0.2 & 6.9 & 0.5 & 8.2 & 0.2 & 5.2 & 0.5 & 0.9 \\
\hline cis-Piperylene & 11.0 & 1.2 & 8.2 & 4.4 & 1.4 & 5.3 & 2.4 & 12.9 & 3.8 & 3.1 & 3.0 & 4.0 \\
\hline 2-Methyl-1-Pentene & 90.5 & 5.7 & 1.4 & 7.2 & 35.4 & 11.9 & 1.0 & 3.9 & 83.1 & 2.4 & 45.3 & 0.9 \\
\hline cis-3-Methyl-2-Pentene & 3.1 & 1.8 & 2.1 & 2.4 & 1.3 & 7.8 & 1.4 & 10.1 & 1.4 & 8.2 & 1.9 & 3.1 \\
\hline 3-Methyl-Hexane & 0.9 & 6.2 & 0.6 & 0.4 & 1.0 & 8.8 & 1.3 & 6.9 & 2.4 & 1.0 & 3.1 & 3.5 \\
\hline 1,4-Pentadiene & 7.2 & 5.6 & 5.8 & 0.6 & 2.0 & 5.2 & 1.8 & 6.8 & 5.4 & 13.0 & 2.4 & 2.4 \\
\hline Methanethiol & 3.3 & 1.4 & 4.3 & 5.2 & 1.9 & 3.3 & 2.3 & 5.7 & 3.3 & 6.4 & 3.7 & 3.8 \\
\hline trans-Piperylene & 1.7 & 7.7 & 1.5 & 9.4 & 0.7 & 0.9 & 0.9 & 7.6 & 2.3 & 2.2 & 1.5 & 5.3 \\
\hline Heptane & 2.3 & 0.1 & 1.4 & 1.8 & 8.7 & 0.0 & 5.3 & 0.1 & 11.3 & 0.5 & 13.9 & 5.2 \\
\hline tert-Butyl Ethyl Ether & 160.7 & 3.7 & 1.2 & 0.4 & 75.9 & 7.5 & 1.0 & 4.1 & 257.5 & 3.0 & 306.6 & 8.2 \\
\hline Ethanal & 70.8 & 2.2 & 65.7 & 0.8 & 11.4 & 12.5 & 17.4 & 4.0 & 56.9 & 3.3 & 67.4 & 1.4 \\
\hline Dimethyl Sulfide & 489.1 & 2.3 & 590.5 & 9.9 & 495.3 & 0.1 & 606.4 & 13.3 & 233.2 & 5.6 & 298.5 & 5.2 \\
\hline 4-Methyl-Heptane & 105.0 & 2.4 & 1.8 & 1.5 & 33.1 & 5.8 & 1.0 & 5.5 & 117.0 & 10.0 & 181.9 & 2.0 \\
\hline Octane & 3.4 & 7.0 & 2.1 & 9.0 & 1.3 & 8.2 & 1.5 & 8.8 & 8.0 & 9.0 & 10.4 & 6.7 \\
\hline Propanal & 8.2 & 5.5 & 5.8 & 2.8 & 4.9 & 9.4 & 3.4 & 4.9 & 24.9 & 13.3 & 18.0 & 5.8 \\
\hline
\end{tabular}




\begin{tabular}{|c|c|c|c|c|c|c|c|c|c|c|c|c|}
\hline Furan & 7.8 & 7.9 & 7.6 & 1.6 & 4.7 & 10.4 & 5.3 & 2.4 & 5.9 & 9.2 & 4.7 & 2.8 \\
\hline 2,4-Dimethyl-Heptane & 17.9 & 2.0 & 20.6 & 6.8 & 13.6 & 2.0 & 21.4 & 5.9 & 11.6 & 3.5 & 12.3 & 8.5 \\
\hline 2-Methyl-Propanal & 83.5 & 3.7 & 90.6 & 1.3 & 22.0 & 0.2 & 21.4 & 2.2 & 26.3 & 0.9 & 28.9 & 1.6 \\
\hline 2-Propanone & 466.4 & 0.1 & 464.8 & 6.9 & 169.6 & 4.9 & 76.0 & 12.8 & 749.5 & 4.9 & 652.7 & 4.0 \\
\hline Methyl Acetate & 5.0 & 4.4 & 7.1 & 5.1 & 0.8 & 3.5 & 1.6 & 3.0 & 0.3 & 1.0 & 0.4 & 3.4 \\
\hline 2-Methyl-2-Heptene & 0.8 & 1.5 & 0.4 & 1.7 & 0.9 & 3.5 & 0.9 & 1.4 & 24.9 & 5.1 & 35.0 & 1.1 \\
\hline Crotonal & 5.1 & 5.2 & 4.4 & 4.8 & 3.3 & 1.8 & 7.6 & 4.0 & 1.8 & 0.2 & 1.8 & 3.6 \\
\hline 2,3-Dimethyl-Heptane & 2.1 & 4.6 & 2.9 & 11.5 & 0.7 & 5.1 & 0.9 & 3.2 & 0.6 & 4.7 & 0.3 & 4.8 \\
\hline 4-Methyl-Octane & 8.9 & 3.5 & 10.4 & 1.3 & 8.5 & 0.6 & 11.1 & 4.3 & 5.7 & 6.7 & 6.7 & 5.7 \\
\hline 2-Methyl-Furan & 1.7 & 8.1 & 1.1 & 7.8 & 1.9 & 3.3 & 1.4 & 12.7 & 1.2 & 11.4 & 1.6 & 11.9 \\
\hline 2,4-Dimethyl-1-Heptene & 12.2 & 0.1 & 10.2 & 6.6 & 27.1 & 6.3 & 10.8 & 1.8 & 344.3 & 8.6 & 428.1 & 1.0 \\
\hline Ethyl Acetate & 65.0 & 5.9 & 71.9 & 6.7 & 9.6 & 4.9 & 16.9 & 9.1 & 46.1 & 7.1 & 64.6 & 1.3 \\
\hline Nonane & 1.6 & 4.3 & 2.6 & 5.5 & 2.6 & 1.4 & 3.7 & 11.6 & 4.6 & 3.4 & 6.4 & 2.4 \\
\hline 2-Butanone & 20.5 & 5.2 & 16.5 & 5.8 & 6.5 & 2.7 & 6.3 & 2.5 & 32.5 & 1.3 & 31.4 & 6.4 \\
\hline 2-Methyl-Butanal & 99.3 & 4.5 & 79.8 & 0.2 & 14.7 & 11.1 & 14.5 & 10.8 & 72.3 & 5.2 & 66.2 & 2.7 \\
\hline 3-Methyl-Butanal & 88.3 & 7.2 & 85.6 & 2.6 & 25.2 & 8.7 & 13.5 & 1.9 & 91.9 & 3.0 & 65.6 & 0.2 \\
\hline Ethyl Alcohol & 556.5 & 4.1 & 661.9 & 3.3 & 907.8 & 2.2 & 1048.8 & 7.8 & 187.7 & 5.9 & 192.0 & 0.5 \\
\hline Benzene & 13.2 & 4.8 & 9.9 & 0.2 & 5.5 & 7.0 & 8.8 & 2.2 & 5.5 & 6.9 & 5.2 & 1.0 \\
\hline 3-Ethyl-Heptane & 0.8 & 3.7 & 0.6 & 0.4 & 1.0 & 3.5 & 1.8 & 14.7 & 8.3 & 3.5 & 6.0 & 2.7 \\
\hline 2-Ethyl-Furan & 16.5 & 3.7 & 38.3 & 2.3 & 18.6 & 6.3 & 20.1 & 13.2 & 21.1 & 8.2 & 16.2 & 3.8 \\
\hline Ethyl Propanoate & 1.3 & 4.7 & 1.9 & 7.6 & 0.2 & 5.9 & 0.2 & 3.3 & 0.8 & 6.1 & 1.0 & 12.3 \\
\hline Ethyl 2-Methylpropanoate & 0.2 & 3.9 & 0.2 & 4.5 & 0.2 & 1.8 & 0.1 & 11.0 & 0.2 & 5.6 & 0.3 & 2.1 \\
\hline 2-Methyl-Crotonal & 7.0 & 5.0 & 6.2 & 2.6 & 3.0 & 4.3 & 2.6 & 7.1 & 1.3 & 3.5 & 1.0 & 3.4 \\
\hline Propyl Acetate & 0.7 & 3.1 & 0.8 & 5.1 & 0.1 & 2.9 & 0.0 & 1.2 & 0.3 & 7.3 & 0.4 & 4.2 \\
\hline 3-Pentanone & 121.4 & 5.0 & 110.6 & 12.2 & 58.4 & 8.1 & 65.6 & 1.1 & 163.9 & 9.3 & 139.6 & 3.8 \\
\hline Pentanal & 1.3 & 1.5 & 1.1 & 12.9 & 1.0 & 1.5 & 0.9 & 4.7 & 14.2 & 9.0 & 11.9 & 4.4 \\
\hline 2,3-Butanedione & 39.5 & 4.2 & 39.0 & 2.0 & 26.9 & 0.5 & 30.7 & 9.9 & 13.2 & 2.0 & 17.0 & 5.8 \\
\hline 2,3,4-Trimethyl-Hexane & 0.1 & 7.4 & 0.2 & 8.3 & 0.5 & 9.0 & 0.5 & 7.9 & 11.3 & 13.2 & 13.8 & 1.8 \\
\hline Decane & 2.2 & 0.3 & 2.0 & 3.1 & 4.8 & 9.7 & 4.9 & 1.2 & 23.5 & 9.5 & 14.2 & 13.1 \\
\hline 2,6-Dimethyl-Nonane & 0.7 & 6.3 & 0.6 & 0.4 & 1.4 & 0.9 & 0.9 & 5.1 & 14.6 & 11.5 & 22.1 & 2 \\
\hline
\end{tabular}




\begin{tabular}{|c|c|c|c|c|c|c|c|c|c|c|c|c|}
\hline 2,5-Dimethyl-Nonane & 0.9 & 3.9 & 1.3 & 9.7 & 1.4 & 13.1 & 1.8 & 1.0 & 0.3 & 1.1 & 0.3 & 6.5 \\
\hline 4-Methyl-2-Pentanone & 1.2 & 0.9 & 0.9 & 3.5 & 0.6 & 3.3 & 0.4 & 0.4 & 29.6 & 2.8 & 21.0 & 5.2 \\
\hline 2-Methy-2-Butanol & 0.1 & 1.0 & 0.2 & 1.3 & 0.5 & 4.3 & 0.3 & 8.7 & 3.5 & 10.7 & 4.2 & 0.7 \\
\hline 2-Methylpropyl Acetate & 2.8 & 2.2 & 5.6 & 2.6 & 0.5 & 4.3 & 0.6 & 1.2 & 0.8 & 9.3 & 1.1 & 0.6 \\
\hline$\alpha$-Pinene & 0.4 & 2.8 & 0.2 & 1.0 & 0.5 & 1.4 & 0.7 & 4.8 & 1.6 & 0.4 & 1.0 & 5.5 \\
\hline 4,4-Dimethyl-2-Pentanone & 0.2 & 0.7 & 0.2 & 2.9 & 0.1 & 11.3 & 0.1 & 3.1 & 4.5 & 7.1 & 4.4 & 3.8 \\
\hline 1-Penten-3-one & 1.9 & 9.1 & 2.1 & 7.8 & 0.9 & 3.9 & 1.3 & 1.4 & 9.5 & 10.1 & 7.7 & 0.7 \\
\hline 5-Butyl-Nonane & 0.5 & 1.7 & 0.6 & 3.2 & 1.3 & 6.8 & 2.4 & 2.0 & 0.6 & 6.8 & 0.8 & 9.5 \\
\hline 3,6-Dimethyl-Decane & 0.2 & 0.1 & 0.1 & 6.2 & 0.2 & 13.9 & 0.3 & 5.5 & 0.1 & 4.7 & 0.1 & 12.0 \\
\hline 2-Methyl-3-Buten-2-ol & 0.7 & 0.7 & 0.6 & 1.6 & 1.4 & 2.1 & 2.0 & 8.0 & 0.8 & 3.7 & 0.7 & 8.4 \\
\hline 5-Methyl-Decane & 0.8 & 1.1 & 1.1 & 5.7 & 0.9 & 3.3 & 1.4 & 1.3 & 0.3 & 3.6 & 0.4 & 1.2 \\
\hline 4-Methyl-Decane & 0.3 & 2.0 & 0.3 & 10.6 & 0.8 & 7.4 & 0.9 & 8.5 & 1.1 & 7.5 & 0.9 & 3.6 \\
\hline 2,3-Pentanedione & 8.4 & 4.0 & 10.2 & 3.4 & 0.8 & 2.8 & 0.6 & 6.9 & 3.3 & 5.0 & 1.9 & 13.3 \\
\hline Methyl 3,3-Dimethylbutanoate & 0.3 & 9.6 & 0.3 & 0.1 & 0.9 & 12.0 & 0.8 & 4.0 & 0.4 & 0.4 & 0.4 & 3.2 \\
\hline 2-Hexanone & 0.8 & 3.3 & 0.5 & 5.4 & 1.2 & 1.3 & 0.9 & 1.6 & 0.9 & 3.1 & 0.8 & 4.4 \\
\hline 4-Methyl-Undecane & 1.8 & 2.8 & 1.1 & 12.2 & 1.6 & 0.8 & 2.8 & 5.7 & 0.6 & 5.1 & 0.7 & 6.6 \\
\hline Hexanal & 37.2 & 0.6 & 21.7 & 2.3 & 0.7 & 2.7 & 0.2 & 4.7 & 75.2 & 0.7 & 59.3 & 10.3 \\
\hline 2-Methyl-1-Propanol & 16.1 & 3.5 & 22.3 & 9.6 & 51.8 & 0.9 & 50.4 & 5.8 & 7.8 & 6.2 & 10.4 & 14.1 \\
\hline 2-Methyl-2-Pentanol & 0.2 & 4.3 & 0.2 & 14.2 & 0.2 & 4.0 & 0.2 & 2.8 & 0.7 & 2.5 & 0.7 & 7.9 \\
\hline Ethyl-Benzene & 25.5 & 4.8 & 29.6 & 3.5 & 3.5 & 1.3 & 44.0 & 2.6 & 17.9 & 8.6 & 17.5 & 3.6 \\
\hline 3-Methylbutyl Acetate & 12.1 & 14.2 & 12.3 & 2.3 & 3.6 & 1.6 & 1.1 & 7.0 & 11.5 & 0.6 & 8.6 & 9.2 \\
\hline o-Xylene & 17.5 & 1.4 & 11.0 & 12.4 & 14.5 & 7.9 & 9.9 & 7.5 & 53.8 & 1.8 & 69.6 & 2.4 \\
\hline 2-Pentanol & 7.9 & 2.1 & 8.2 & 2.4 & 7.7 & 1.7 & 6.3 & 3.9 & 2.1 & 4.6 & 3.3 & 11.4 \\
\hline p-Xylene & 36.6 & 1.1 & 38.4 & 8.7 & 28.3 & 0.7 & 30.6 & 3.0 & 39.9 & 1.5 & 29.2 & 5.5 \\
\hline 5-Methyl-2-Hexanone & 0.3 & 4.9 & 0.4 & 5.1 & 0.5 & 3.3 & 0.3 & 6.5 & 1.3 & 0.2 & 1.7 & 10.9 \\
\hline Methyl 2-Ethylpentanoate & 12.0 & 0.5 & 8.5 & 2.6 & 4.4 & 4.0 & 5.8 & 9.1 & 28.3 & 7.7 & 21.2 & 4.7 \\
\hline 1-Butanol & 0.4 & 1.6 & 0.5 & 7.5 & 11.5 & 2.9 & 18.5 & 8.7 & 0.5 & 4.4 & 0.3 & 3.6 \\
\hline$\alpha$-Phellandrene & 5.8 & 4.1 & 7.8 & 1.6 & 1.6 & 2.6 & 2.5 & 3.2 & 174.9 & 1.3 & 87.5 & 5.2 \\
\hline 2-Methyl-3-Pentanol & 9.2 & 2.0 & 12.8 & 7.4 & 4.0 & 8.4 & 3.6 & 3.6 & 3.3 & 10.1 & 2.8 & 9.6 \\
\hline 1-Penten-3-ol & 38.5 & 2.9 & 45.3 & 8.8 & 95.2 & 5.7 & 112.8 & 3.4 & 180.5 & 12.7 & 112.5 & 1.5 \\
\hline
\end{tabular}




\begin{tabular}{|c|c|c|c|c|c|c|c|c|c|c|c|c|}
\hline$\alpha$-Terpinene & 5.0 & 3.8 & 7.8 & 0.4 & 0.4 & 2.2 & 0.6 & 2.9 & 40.5 & 6.3 & 29.6 & 7.4 \\
\hline Butyl Prop-2-enoate & 3.6 & 2.9 & 5.1 & 1.4 & 4.5 & 1.7 & 6.5 & 10.3 & 16.6 & 5.0 & 9.1 & 8.2 \\
\hline m-Xylene & 1.5 & 2.7 & 1.9 & 11.9 & 12.0 & 0.6 & 16.5 & 0.1 & 30.9 & 1.7 & 23.0 & 5.5 \\
\hline 2-Heptanone & 225.8 & 13.6 & 145.9 & 4.3 & 504.1 & 3.0 & 620.8 & 0.8 & 312.3 & 14.2 & 345.7 & 3.2 \\
\hline Heptanal & 0.8 & 6.8 & 0.8 & 3.2 & 1.7 & 3.3 & 1.3 & 10.8 & 4.9 & 12.8 & 4.1 & 2.0 \\
\hline D-Limonene & 0.2 & 2.5 & 0.1 & 1.5 & 1.3 & 10.4 & 1.6 & 8.9 & 1.8 & 10.3 & 1.3 & 3.9 \\
\hline 3-Hexanol & 3.2 & 2.6 & 3.5 & 8.4 & 1.7 & 2.2 & 1.9 & 7.1 & 0.8 & 3.5 & 0.5 & 3.5 \\
\hline$\beta$-Phellandrene & 2.0 & 3.4 & 4.3 & 4.0 & 9.2 & 0.3 & 10.0 & 10.5 & 392.9 & 6.1 & 316.7 & 5.1 \\
\hline Eucalyptol & 0.3 & 5.1 & 0.4 & 1.6 & 0.1 & 3.4 & 0.1 & 1.2 & 0.8 & 1.2 & 0.5 & 4.0 \\
\hline m-Menthane & 0.3 & 6.6 & 0.4 & 4.0 & 0.5 & 4.9 & 0.7 & 5.4 & 2.1 & 8.1 & 2.6 & 2.6 \\
\hline 4-Methyl-2-Heptanone & 2.4 & 8.8 & 2.1 & 1.6 & 1.9 & 2.9 & 1.6 & 6.4 & 409.5 & 10.5 & 436.8 & 0.2 \\
\hline 3-Methyl-2-Pentanol & 5.1 & 3.3 & 7.1 & 2.8 & 89.7 & 0.3 & 92.9 & 8.8 & 1.8 & 4.4 & 3.7 & 5.4 \\
\hline 2-Methyl-1-Butanol & 83.9 & 4.8 & 119.9 & 6.7 & 139.2 & 9.5 & 182.6 & 1.8 & 18.0 & 8.5 & 25.5 & 3.6 \\
\hline 3-Methyl-1-Butanol & 278.0 & 5.4 & 418.2 & 6.7 & 314.8 & 1.3 & 564.4 & 5.7 & 32.0 & 10.2 & 35.4 & 5.1 \\
\hline o-Ethyltoluene & 6.5 & 3.6 & 5.4 & 2.7 & 2.1 & 2.5 & 3.8 & 6.1 & 6.5 & 7.0 & 6.2 & 4.4 \\
\hline m-Ethyltoluene & 7.0 & 3.8 & 9.5 & 4.0 & 6.1 & 0.7 & 7.1 & 2.8 & 2.7 & 9.5 & 2.0 & 5.4 \\
\hline 2-Hexanol & 3.1 & 1.2 & 1.8 & 12.8 & 5.5 & 1.0 & 8.6 & 3.8 & 1.9 & 5.3 & 1.8 & 2.2 \\
\hline Ethylene Glycol Ethyl Ether & 9.2 & 1.9 & 18.9 & 6.0 & 2.4 & 11.3 & 1.8 & 1.7 & 1.4 & 3.0 & 2.3 & 13.3 \\
\hline 6-Methyl-2-Heptanone & 2.0 & 1.4 & 1.7 & 5.2 & 2.0 & 5.3 & 1.4 & 1.7 & 8.1 & 15.2 & 7.5 & 1.8 \\
\hline Pseudocumene & 4.4 & 2.5 & 5.3 & 0.1 & 2.2 & 1.4 & 3.8 & 7.3 & 4.9 & 5.8 & 5.1 & 11.3 \\
\hline 4,6-Dimethyl-2-Heptanone & 0.9 & 5.1 & 0.8 & 4.7 & 0.4 & 0.1 & 0.3 & 2.8 & 43.8 & 10.9 & 59.8 & 2.2 \\
\hline 2,3-Dimethyl-2-Hexanol & 0.2 & 10.3 & 0.3 & 3.7 & 0.2 & 11.0 & 0.4 & 8.4 & 20.1 & 7.2 & 19.1 & 4.2 \\
\hline 3-Octanone & 1.0 & 2.7 & 0.8 & 2.2 & 17.1 & 0.8 & 21.5 & 0.7 & 0.4 & 9.7 & 0.3 & 3.9 \\
\hline Styrene & 48.5 & 2.3 & 64.2 & 1.0 & 49.0 & 1.8 & 58.1 & 13.0 & 24.0 & 4.3 & 30.6 & 0.1 \\
\hline 5-Methyl-2-Heptanone & 10.1 & 5.4 & 9.6 & 5.3 & 7.4 & 0.2 & 8.0 & 7.3 & 18.5 & 8.8 & 26.4 & 0.5 \\
\hline 1-Pentanol & 28.7 & 4.9 & 47.3 & 1.8 & 6.7 & 5.8 & 8.8 & 2.0 & 4.8 & 1.8 & 5.9 & 2.6 \\
\hline p-Ethyltoluene & 4.5 & 1.7 & 5.3 & 2.7 & 3.2 & 6.4 & 4.5 & 1.7 & 2.6 & 5.1 & 1.9 & 3.6 \\
\hline p-Cymene & 11.8 & 3.8 & 17.1 & 6.0 & 3.4 & 6.8 & 3.7 & 1.8 & 46.6 & 11.9 & 32.3 & 4.4 \\
\hline Mesitylene & 8.6 & 4.3 & 11.7 & 0.8 & 14.2 & 1.1 & 15.2 & 0.1 & 9.8 & 13.4 & 8.3 & 4.4 \\
\hline 4-Methyl-4-Heptanol & 0.4 & 9.8 & 0.4 & 3.5 & 0.4 & 7.4 & 0.2 & 1.4 & 1.0 & 6.8 & 0.7 & 0.3 \\
\hline
\end{tabular}




\begin{tabular}{|c|c|c|c|c|c|c|c|c|c|c|c|c|}
\hline 4-Heptanol & 3.9 & 0.3 & 3.5 & 4.1 & 1.9 & 8.9 & 1.4 & 4.1 & 17.6 & 12.2 & 10.5 & 0.2 \\
\hline 2-Octanone & 40.1 & 9.1 & 29.0 & 4.2 & 73.0 & 8.1 & 82.0 & 2.4 & 75.3 & 13.1 & 88.0 & 9.2 \\
\hline Octanal & 1.5 & 7.3 & 2.2 & 8.1 & 1.6 & 5.0 & 1.8 & 5.6 & 4.3 & 10.3 & 4.0 & 6.2 \\
\hline 3-Hydroxy-2-Butanone & 0.2 & 9.0 & 0.2 & 7.5 & 0.3 & 9.1 & 0.2 & 5.9 & 0.1 & 3.0 & 0.1 & 2.0 \\
\hline 1-Octen-3-one & 3.4 & 0.2 & 4.1 & 6.8 & 0.2 & 14.3 & 0.2 & 12.0 & 23.5 & 0.7 & 15.0 & 0.8 \\
\hline trans-2-Pentenol & 15.7 & 0.3 & 23.6 & 10.3 & 14.7 & 2.6 & 22.5 & 1.1 & 14.9 & 7.6 & 17.2 & 2.7 \\
\hline 2,2,6-Trimethyl-Cyclohexanone & 1.9 & 0.8 & 2.2 & 5.1 & 2.7 & 6.5 & 3.2 & 10.4 & 3.9 & 5.2 & 3.7 & 1.0 \\
\hline 2-Heptanol & 653.4 & 1.9 & 601.6 & 6.7 & 629.7 & 5.4 & 755.5 & 0.7 & 435.8 & 9.0 & 411.3 & 9.4 \\
\hline cis-2-Pentenol & 209.5 & 0.1 & 293.4 & 7.5 & 95.3 & 2.2 & 132.1 & 11.4 & 147.1 & 5.5 & 128.5 & 5.2 \\
\hline 1-Hydroxy-2-Propanone & 90.8 & 3.9 & 120.8 & 5.1 & 6.1 & 0.8 & 4.4 & 2.6 & 4.0 & 9.7 & 4.6 & 5.3 \\
\hline 5-Methyl-4-Hexen-3-one & 2.2 & 0.2 & 2.6 & 5.2 & 3.1 & 3.9 & 3.4 & 1.5 & 2.0 & 8.9 & 1.7 & 3.4 \\
\hline Hemimellitene & 5.7 & 3.2 & 5.4 & 1.7 & 3.7 & 7.7 & 4.6 & 2.4 & 3.8 & 17.4 & 2.7 & 5.2 \\
\hline 6-Methyl-5-Hepten-2-one & 10.2 & 1.7 & 15.6 & 8.9 & 9.4 & 2.4 & 13.4 & 8.1 & 43.7 & 5.2 & 41.4 & 2.7 \\
\hline 2-Pentene & 452.5 & 1.0 & 338.5 & 13.1 & 495.7 & 2.2 & 476.8 & 0.2 & 346.6 & 11.1 & 430.6 & 1.1 \\
\hline 1-Hexanol & 650.6 & 2.7 & 742.2 & 0.6 & 360.8 & 0.4 & 462.9 & 2.2 & 115.5 & 11.4 & 191.5 & 0.6 \\
\hline 2-Cyclopenten-1-one & 14.6 & 9.1 & 15.4 & 8.4 & 1.5 & 5.5 & 2.0 & 4.5 & 9.1 & 10.6 & 13.9 & 9.0 \\
\hline 2-Methyl-2-Cyclopenten-1-one & 3.7 & 6.8 & 3.4 & 7.8 & 6.1 & 4.4 & 0.6 & 7.7 & 4.2 & 6.3 & 5.0 & 4.1 \\
\hline$\beta$-Methy-Styrene & 3.3 & 1.8 & 3.4 & 1.3 & 2.3 & 1.5 & 2.8 & 12.9 & 0.8 & 6.5 & 0.7 & 0.1 \\
\hline 4-Octanol & 3.9 & 2.4 & 3.4 & 9.7 & 3.4 & 6.3 & 4.3 & 0.9 & 6.3 & 5.0 & 5.2 & 0.1 \\
\hline cis-3-Hexen-1-ol & 5.4 & 3.6 & 7.2 & 0.3 & 4.3 & 0.3 & 5.2 & 0.7 & 1.6 & 5.5 & 1.6 & 5.4 \\
\hline 2-Nonanone & 12.7 & 2.0 & 20.0 & 7.9 & 146.7 & 2.2 & 261.6 & 5.7 & 1.4 & 5.5 & 1.2 & 0.9 \\
\hline Nonanal & 38.8 & 3.7 & 48.1 & 7.4 & 36.8 & 1.2 & 48.7 & 5.2 & 4.2 & 1.6 & 3.2 & 14.1 \\
\hline Ethylene Glycol Butyl Ether & 22.7 & 3.8 & 23.5 & 2.1 & 5.5 & 7.5 & 5.4 & 4.3 & 1.7 & 5.8 & 1.3 & 11.0 \\
\hline 1-Ethyl-1-Methyl-Cyclopentane & 7.3 & 5.4 & 6.6 & 7.3 & 4.7 & 0.6 & 4.0 & 14.9 & 64.5 & 9.5 & 52.0 & 5.0 \\
\hline 3-Octen-2-one & 8.0 & 4.2 & 8.7 & 4.1 & 2.4 & 12.1 & 3.0 & 3.5 & 36.5 & 5.5 & 30.2 & 5.2 \\
\hline 2-Octanol & 112.0 & 1.4 & 82.7 & 0.6 & 251.1 & 3.7 & 298.1 & 3.4 & 52.4 & 14.0 & 55.2 & 5.8 \\
\hline 3-Ethyl-2-Methyl-1,3-Hexadiene & 0.3 & 2.7 & 0.3 & 10.6 & 2.4 & 0.9 & 2.5 & 5.4 & 0.2 & 2.2 & 0.3 & 14.3 \\
\hline 3-Bis(1,1-Dimethylethyl)-Benzene & 0.2 & 11.3 & 0.2 & 4.5 & 0.5 & 1.1 & 0.4 & 10.5 & 29.5 & 13.2 & 37.1 & 7.2 \\
\hline trans-2-Octenal & 2.4 & 2.0 & 1.5 & 3.3 & 9.7 & 0.4 & 9.8 & 1.0 & 35.9 & 9.8 & 27.8 & 6.6 \\
\hline m-Ethyl-Styrene & 0.5 & 1.6 & 0.4 & 5.5 & 2.6 & 5.2 & 3.6 & 9.6 & 13.5 & 9.5 & 21.8 & 9.5 \\
\hline
\end{tabular}




\begin{tabular}{|c|c|c|c|c|c|c|c|c|c|c|c|c|}
\hline 5-Methyl-2-Heptanone & 0.8 & 4.4 & 0.7 & 1.1 & 0.5 & 1.7 & 0.5 & 12.9 & 19.2 & 13.3 & 13.6 & 3.2 \\
\hline 2-Decanone & 1.7 & 1.5 & 1.6 & 6.9 & 6.5 & 0.9 & 7.9 & 6.5 & 11.9 & 14.1 & 18.6 & 12.2 \\
\hline 1-Octen-3-ol & 198.9 & 3.3 & 168.5 & 12.9 & 156.8 & 1.5 & 115.8 & 5.7 & 328.2 & 11.5 & 264.8 & 4.9 \\
\hline trans,trans-2,4-Heptadienal & 4.8 & 1.8 & 5.5 & 6.1 & 3.6 & 6.0 & 3.1 & 2.1 & 1.7 & 10.4 & 1.3 & 1.6 \\
\hline 1-Heptanol & 79.3 & 7.9 & 87.7 & 2.2 & 85.0 & 6.6 & 85.1 & 4.7 & 13.1 & 2.3 & 16.5 & 10.4 \\
\hline cis,cis-3,5-Octadiene & 1.0 & 10.4 & 0.9 & 1.6 & 1.8 & 0.3 & 2.6 & 5.1 & 0.7 & 4.9 & 0.9 & 5.3 \\
\hline Methyl 2-Oxopropanoate & 153.9 & 2.9 & 203.9 & 4.7 & 108.5 & 6.8 & 99.1 & 0.7 & 55.8 & 4.0 & 48.8 & 0.8 \\
\hline Dihydromyrcenol & 15.3 & 7.0 & 19.1 & 2.8 & 12.6 & 2.0 & 11.3 & 6.9 & 12.1 & 6.2 & 9.9 & 1.2 \\
\hline trans-Menthone & 18.0 & 4.2 & 25.6 & 14.1 & 1.8 & 1.9 & 2.2 & 1.5 & 82.3 & 11.3 & 67.6 & 4.4 \\
\hline Furfural & 78.6 & 3.5 & 76.0 & 8.7 & 82.4 & 4.8 & 84.4 & 2.3 & 31.9 & 7.3 & 36.5 & 0.6 \\
\hline Ethanoic Acid & 1925.2 & 1.5 & 2142.7 & 0.1 & 523.1 & 2.2 & 534.2 & 0.8 & 19.4 & 1.7 & 11.9 & 8.8 \\
\hline 2-Ethyl-1-Hexanol & 20.7 & 2.5 & 28.4 & 1.5 & 13.7 & 12.9 & 17.2 & 10.3 & 44.1 & 12.8 & 49.0 & 2.9 \\
\hline Decanal & 59.8 & 6.2 & 64.6 & 4.5 & 26.0 & 3.4 & 20.7 & 0.3 & 45.9 & 4.9 & 38.4 & 1.5 \\
\hline Furfuryl Formate & 60.9 & 1.8 & 67.0 & 0.6 & 10.0 & 3.7 & 14.7 & 9.7 & 9.5 & 4.1 & 8.7 & 2.9 \\
\hline trans-2-Hepten-1-ol & 49.0 & 5.3 & 48.8 & 5.5 & 24.5 & 2.5 & 26.4 & 3.9 & 10.6 & 6.2 & 15.9 & 7.8 \\
\hline 2-Nonanol & 21.4 & 1.4 & 22.0 & 1.8 & 351.5 & 1.6 & 413.3 & 2.1 & 47.1 & 2.5 & 52.1 & 9.7 \\
\hline 1-(2-Furanyl)-Ethanone & 63.0 & 9.3 & 70.5 & 2.9 & 46.7 & 7.0 & 47.8 & 1.3 & 33.6 & 3.6 & 32.3 & 6.3 \\
\hline 3,5-Octadien-2-one & 19.0 & 0.7 & 23.9 & 10.9 & 8.9 & 2.3 & 11.0 & 2.9 & 36.4 & 4.7 & 29.4 & 5.8 \\
\hline Benzaldehyde & 22.8 & 1.0 & 26.2 & 3.2 & 25.1 & 11.1 & 23.6 & 1.1 & 33.4 & 2.6 & 41.9 & 0.8 \\
\hline Furfuryl Acetate & 0.5 & 1.7 & 0.5 & 5.0 & 0.2 & 4.9 & 0.2 & 8.4 & 0.3 & 4.6 & 2.5 & 2.9 \\
\hline Linalool & 1.5 & 8.6 & 1.3 & 0.9 & 1.2 & 8.7 & 1.2 & 12.5 & 0.9 & 12.7 & 1.1 & 4.2 \\
\hline$\beta$-Myrcene & 0.2 & 1.7 & 0.2 & 1.4 & 1.3 & 4.4 & 1.2 & 11.1 & 5.2 & 3.8 & 4.7 & 0.7 \\
\hline 1-Octanol & 95.4 & 3.8 & 103.2 & 12.7 & 89.0 & 3.2 & 106.5 & 6.5 & 34.3 & 4.5 & 32.9 & 3.8 \\
\hline trans-p-2-Menthen-1-ol & 52.9 & 4.2 & 73.4 & 2.6 & 2.2 & 0.4 & 2.9 & 4.7 & 111.0 & 11.1 & 91.4 & 5.0 \\
\hline Propanoic Acid & 18.5 & 2.0 & 18.6 & 8.2 & 0.5 & 3.0 & 0.5 & 11.8 & 11.2 & 5.0 & 8.8 & 5.5 \\
\hline 2,3-Butanediol & 67.1 & 4.0 & 75.5 & 1.7 & 133.3 & 2.7 & 267.7 & 11.5 & 57.3 & 6.8 & 51.8 & 1.6 \\
\hline Dimethyl Sulfoxide & 99.9 & 0.1 & 103.7 & 3.3 & 50.3 & 2.2 & 69.5 & 5.4 & 5.6 & 1.9 & 5.3 & 10.7 \\
\hline m-Menth-3-ene & 31.5 & 0.4 & 45.9 & 5.5 & 13.8 & 7.1 & 12.8 & 8.5 & 6.2 & 4.0 & 7.0 & 1.7 \\
\hline s-(2-Ethylcyclopentyl)-Methanol & 39.2 & 2.8 & 40.8 & 6.3 & 32.0 & 0.7 & 38.1 & 9.6 & 10.7 & 5.2 & 14.2 & 1.0 \\
\hline Bornyl Acetate & 8.0 & 3.9 & 9.4 & 3.2 & 9.4 & 1.9 & 11.0 & 4.7 & 0.9 & 7.5 & 1.0 & 7.1 \\
\hline
\end{tabular}




\begin{tabular}{|c|c|c|c|c|c|c|c|c|c|c|c|c|}
\hline 4-Cyclopentene-1,3-dione & 79.7 & 3.3 & 99.1 & 2.8 & 19.1 & 9.1 & 14.0 & 8.0 & 39.9 & 1.5 & 48.2 & 7.0 \\
\hline Menthol & 3.1 & 3.7 & 3.1 & 10.5 & 2.6 & 0.9 & 2.6 & 1.0 & 45.2 & 1.0 & 42.3 & 5.4 \\
\hline 2-Undecanone & 2.4 & 4.9 & 3.3 & 0.9 & 1.0 & 3.8 & 1.8 & 11.8 & 2.4 & 7.8 & 1.7 & 3.7 \\
\hline 2-(2-Furanyl)-Furan & 1.5 & 2.4 & 2.3 & 11.0 & 1.1 & 4.7 & 0.9 & 10.7 & 0.5 & 4.2 & 0.6 & 3.6 \\
\hline trans-2-Octen-1-ol & 63.2 & 0.1 & 72.5 & 0.1 & 50.7 & 0.1 & 53.4 & 5.1 & 15.0 & 5.1 & 15.1 & 12.8 \\
\hline trans-2-Nonen-1-ol & 26.8 & 6.4 & 28.8 & 4.7 & 22.4 & 6.9 & 25.7 & 6.5 & 68.9 & 5.4 & 63.3 & 8.3 \\
\hline Diethylene Glycol Ethyl Ether & 56.8 & 2.5 & 60.8 & 3.4 & 50.0 & 9.5 & 43.5 & 2.3 & 53.9 & 14.4 & 60.4 & 1.9 \\
\hline 5-Trimethyl-2-Cyclopenten-1-one & 18.3 & 5.4 & 16.8 & 2.3 & 17.1 & 5.7 & 11.6 & 0.7 & 12.9 & 12.6 & 15.7 & 6.2 \\
\hline cis-p-2-Menthen-1-ol & 2.7 & 0.8 & 33.7 & 6.6 & 3.4 & 0.3 & 3.9 & 3.9 & 5.0 & 6.4 & 4.2 & 0.5 \\
\hline$\beta$-Cyclocitral & 9.6 & 6.7 & 8.8 & 3.0 & 6.6 & 3.7 & 5.9 & 4.5 & 19.7 & 10.5 & 15.4 & 3.1 \\
\hline Benzeneacetaldehyde & 14.6 & 4.9 & 17.8 & 0.7 & 13.6 & 3.2 & 12.7 & 9.2 & 80.9 & 1.5 & 77.3 & 2.1 \\
\hline 1-Nonanol & 28.2 & 5.6 & 31.1 & 5.1 & 75.8 & 2.5 & 84.7 & 0.0 & 9.1 & 4.1 & 9.6 & 2.9 \\
\hline Furfuryl Alcohol & 42.7 & 6.5 & 51.2 & 5.4 & 13.2 & 2.0 & 12.1 & 14.8 & 52.0 & 5.2 & 55.0 & 0.9 \\
\hline Phoracanthol & 12.5 & 12.3 & 13.5 & 3.1 & 9.7 & 2.6 & 10.5 & 9.8 & 5.6 & 5.7 & 6.3 & 3.4 \\
\hline cis-3-Nonen-1-ol & 16.6 & 6.1 & 21.2 & 7.0 & 14.6 & 14.1 & 14.1 & 11.2 & 15.3 & 7.6 & 12.7 & 10.4 \\
\hline cis-Piperitol & 27.4 & 4.4 & 29.3 & 7.3 & 2.3 & 2.1 & 2.8 & 1.9 & 46.0 & 4.3 & 39.7 & 5.4 \\
\hline p-Methoxy-Styrene & 4.2 & 5.3 & 5.2 & 7.7 & 0.3 & 3.2 & 0.4 & 1.9 & 2.8 & 3.0 & 3.5 & 0.9 \\
\hline (2Z)-3-Ethyl-2,4-Pentadien-1-ol & 7.7 & 6.8 & 7.2 & 15.3 & 4.3 & 2.1 & 4.5 & 6.9 & 1.3 & 1.9 & 1.7 & 13.8 \\
\hline Butylene Glycol Monobutyl Ether & 10.8 & 0.5 & 15.3 & 10.9 & 8.4 & 1.7 & 8.8 & 3.5 & 1.4 & 0.3 & 1.2 & 3.6 \\
\hline$\alpha$-Terpineol & 0.5 & 6.5 & 0.5 & 6.2 & 0.2 & 16.4 & 0.2 & 10.0 & 5.6 & 3.0 & 4.5 & 2.7 \\
\hline 1,5-Cyclooctanedione & 2.4 & 13.6 & 2.9 & 2.6 & 2.5 & 11.9 & 2.9 & 0.9 & 4.8 & 5.9 & 4.2 & 1.9 \\
\hline$\gamma$-Terpinene & 3.1 & 4.1 & 3.6 & 6.1 & 4.6 & 4.2 & 5.5 & 3.3 & 1.8 & 7.7 & 1.6 & 0.8 \\
\hline trans-2-Decenol & 11.3 & 1.8 & 11.6 & 5.7 & 6.6 & 0.8 & 6.7 & 1.7 & 3.2 & 0.7 & 3.6 & 0.7 \\
\hline 2-Propenoic Acid & 0.2 & 8.1 & 0.2 & 2.7 & 0.5 & 2.0 & 0.5 & 1.6 & 0.9 & 1.5 & 1.3 & 4.6 \\
\hline 2-Undecanol & 0.9 & 2.7 & 1.1 & 4.0 & 21.9 & 0.7 & 27.2 & 0.9 & 2.5 & 7.5 & 1.9 & 12.3 \\
\hline 3-Ethyl-Benzaldehyde & 5.0 & 2.9 & 5.5 & 2.6 & 3.2 & 1.0 & 3.9 & 12.7 & 6.2 & 4.6 & 6.9 & 5.9 \\
\hline 3-Hydroxy-Benzaldehyde & 11.9 & 1.7 & 13.5 & 9.7 & 0.9 & 0.2 & 1.0 & 4.6 & 1.5 & 2.8 & 1.7 & 4.3 \\
\hline trans-2-Nonene & 1.5 & 2.8 & 1.5 & 8.8 & 1.6 & 0.7 & 1.1 & 8.2 & 1.8 & 3.5 & 1.8 & 8.9 \\
\hline 2-Methyl-2-Pentenal & 9.0 & 3.1 & 11.6 & 15.1 & 3.7 & 2.2 & 4.0 & 2.4 & 9.5 & 12.1 & 10.0 & 11.3 \\
\hline cis-5-Decenol & 4.5 & 1.1 & 4.3 & 6.0 & 88.0 & 3.3 & 88.9 & 6.3 & 18.3 & 9.1 & 14.0 & 1.9 \\
\hline
\end{tabular}




\begin{tabular}{|c|c|c|c|c|c|c|c|c|c|c|c|c|}
\hline trans-Piperitol & 20.2 & 3.3 & 24.9 & 7.3 & 2.0 & 6.5 & 2.2 & 7.0 & 35.6 & 1.4 & 31.8 & 5.4 \\
\hline 4-Ethyl-Benzaldehyde & 3.4 & 0.6 & 3.4 & 1.9 & 3.4 & 0.2 & 3.3 & 4.2 & 4.4 & 12.5 & 5.0 & 1.3 \\
\hline Naphthalene & 13.2 & 3.2 & 16.0 & 13.1 & 10.7 & 3.6 & 9.0 & 10.5 & 1.3 & 9.6 & 1.4 & 9.1 \\
\hline 1-Decanol & 14.0 & 1.0 & 17.0 & 7.3 & 19.0 & 9.1 & 22.6 & 0.7 & 19.2 & 5.8 & 19.3 & 6.0 \\
\hline Cyclodecane & 67.6 & 3.7 & 71.7 & 3.4 & 34.7 & 3.1 & 33.4 & 3.4 & 41.8 & 6.0 & 42.3 & 3.4 \\
\hline trans,trans-2,4-Decadienal & 16.0 & 7.5 & 18.5 & 12.7 & 19.0 & 13.0 & 22.4 & 4.7 & 4.1 & 6.0 & 4.4 & 0.6 \\
\hline 1,2-Cyclopentanedione & 567.8 & 2.4 & 626.0 & 2.9 & 238.4 & 3.8 & 213.0 & 1.6 & 264.4 & 1.9 & 216.1 & 1.5 \\
\hline Cyclodecene & 3.3 & 2.0 & 3.3 & 8.2 & 1.9 & 1.5 & 1.8 & 13.4 & 0.5 & 3.1 & 0.6 & 2.4 \\
\hline cis-4-Decenol & 0.7 & 2.9 & 0.8 & 1.4 & 1.2 & 3.1 & 1.5 & 4.0 & 2.8 & 4.5 & 2.9 & 9.0 \\
\hline 1-Phenyl-2-Butanone & 6.0 & 1.8 & 7.5 & 10.8 & 13.5 & 2.2 & 12.6 & 0.9 & 8.8 & 5.6 & 8.4 & 6.1 \\
\hline Phenethyl Alcohol & 20.4 & 1.0 & 22.2 & 11.7 & 4.2 & 1.8 & 4.3 & 0.2 & 9.6 & 1.4 & 8.0 & 5.5 \\
\hline trans,cis-2,4-Decadienal & 12.4 & 4.8 & 13.5 & 13.5 & 11.5 & 6.2 & 16.6 & 5.0 & 3.2 & 2.3 & 3.1 & 5.1 \\
\hline 2-Cyclohexenol & 66.3 & 3.6 & 77.1 & 1.0 & 10.9 & 3.0 & 12.4 & 1.5 & 8.2 & 1.7 & 8.6 & 5.0 \\
\hline 2-Ethyl Hexanoic Acid & 16.6 & 9.7 & 20.6 & 8.0 & 1.1 & 7.7 & 1.0 & 1.3 & 17.4 & 1.4 & 14.1 & 4.4 \\
\hline 3-Methyl-1,2-Cyclopentanedione & 64.5 & 1.9 & 70.1 & 5.2 & 2.2 & 7.2 & 2.8 & 0.6 & 7.0 & 4.8 & 7.3 & 3.2 \\
\hline trans-Cinnamaldehyde & 9.4 & 2.8 & 9.1 & 4.0 & 4.8 & 1.4 & 4.9 & 4.4 & 8.3 & 5.0 & 7.7 & 3.3 \\
\hline Heptanoic Acid & 48.2 & 0.3 & 57.1 & 8.2 & 1.8 & 7.7 & 1.3 & 0.9 & 103.3 & 7.5 & 106.1 & 9.7 \\
\hline Mequinol & 90.6 & 9.8 & 129.2 & 2.3 & 45.4 & 0.8 & 48.1 & 6.8 & 7.5 & 2.8 & 8.0 & 6.4 \\
\hline 2,4,5-Trihydroxypyrimidine & 8.2 & 8.8 & 8.9 & 1.9 & 16.3 & 7.6 & 11.9 & 6.7 & 15.6 & 8.1 & 18.1 & 11.6 \\
\hline cis-Cinnamaldehyde & 8.2 & 1.1 & 8.8 & 2.3 & 4.3 & 6.8 & 4.7 & 7.0 & 8.6 & 5.6 & 8.5 & 7.9 \\
\hline Benzenemethanol & 82.4 & 2.7 & 84.9 & 8.8 & 40.0 & 1.2 & 43.5 & 11.9 & 18.5 & 11.3 & 11.2 & 3.7 \\
\hline Mesitaldehyde & 6.0 & 1.2 & 6.0 & 0.9 & 3.2 & 9.9 & 3.6 & 9.4 & 6.4 & 7.0 & 6.0 & 5.6 \\
\hline 2-Ethylhexyl Octanoate & 9.2 & 3.7 & 9.8 & 2.9 & 4.3 & 3.0 & 4.7 & 7.9 & 4.4 & 0.7 & 3.9 & 0.7 \\
\hline Benzeneethanol & 61.1 & 0.9 & 65.9 & 10.8 & 29.4 & 4.0 & 32.8 & 6.2 & 42.4 & 11.0 & 44.7 & 6.1 \\
\hline 1,4-Butanediol & 69.1 & 8.2 & 67.2 & 7.1 & 37.1 & 3.6 & 40.7 & 6.0 & 48.7 & 3.1 & 54.5 & 9.2 \\
\hline$\beta$-Ionone & 28.8 & 8.6 & 22.1 & 11.9 & 19.1 & 14.0 & 18.5 & 3.6 & 56.0 & 13.9 & 49.4 & 1.2 \\
\hline 2-Methyl-1,4-Benzenediol & 20.3 & 3.6 & 25.9 & 8.6 & 7.1 & 2.4 & 0.8 & 7.2 & 1.6 & 13.1 & 2.0 & 0.4 \\
\hline 2-Ethyl Heptanoic Acid & 2.4 & 2.6 & 2.9 & 7.1 & 10.5 & 5.7 & 15.3 & 12.8 & 1.8 & 14.0 & 1.9 & 13.5 \\
\hline 1-Dodecanol & 3.7 & 6.5 & 3.3 & 10.4 & 6.0 & 4.2 & 5.5 & 2.2 & 13.1 & 3.2 & 12.0 & 5.4 \\
\hline Benzalacetone & 8.8 & 1.7 & 10.0 & 2.9 & 5.3 & 7.5 & 5.1 & 2.2 & 10.7 & 5.1 & 11.9 & 1.6 \\
\hline
\end{tabular}




\begin{tabular}{|c|c|c|c|c|c|c|c|c|c|c|c|c|}
\hline Maltol & 186.6 & 2.8 & 239.2 & 3.1 & 2.7 & 1.4 & 22.7 & 3.1 & 77.1 & 2.2 & 72.3 & 7.9 \\
\hline 2-Acetylpyrrole & 13.1 & 7.7 & 12.5 & 8.6 & 1.1 & 10.6 & 1.0 & 9.5 & 2.6 & 6.7 & 2.3 & 13.4 \\
\hline 2,4-Decadien-1-ol & 8.9 & 0.4 & 9.7 & 6.9 & 8.8 & 4.7 & 9.8 & 2.4 & 4.6 & 1.8 & 4.0 & 0.7 \\
\hline Levoglucosenone & 17.0 & 0.5 & 15.1 & 10.4 & 1.0 & 8.3 & 1.4 & 6.5 & 4.3 & 1.9 & 4.0 & 6.6 \\
\hline Phenol & 26.9 & 7.6 & 22.3 & 9.3 & 38.4 & 6.4 & 41.7 & 7.0 & 37.9 & 6.7 & 39.4 & 0.0 \\
\hline 1-(2-Furanyl)-2-Hydroxy-Ethanone & 9.6 & 4.4 & 10.0 & 2.1 & 24.5 & 1.7 & 26.2 & 3.8 & 6.0 & 1.1 & 7.0 & 1.4 \\
\hline 4,6-Dimethyl-Dodecane & 28.3 & 0.6 & 27.8 & 12.7 & 1.4 & 2.6 & 1.9 & 6.2 & 10.9 & 8.5 & 11.8 & 7.1 \\
\hline Ethyl Decanoate & 6.1 & 5.7 & 7.0 & 6.4 & 9.2 & 3.1 & 10.0 & 0.8 & 1.4 & 1.1 & 1.8 & 12.2 \\
\hline 1-Methyl-4-Piperidinone & 1.9 & 5.1 & 1.5 & 13.6 & 0.7 & 1.3 & 0.7 & 1.6 & 4.7 & 3.0 & 4.3 & 3.2 \\
\hline 2,2-Dimethyl-3-Heptanone & 102.9 & 4.4 & 183.6 & 8.3 & 1.4 & 4.6 & 1.0 & 5.2 & 38.5 & 6.1 & 37.4 & 3.1 \\
\hline Octanoic Acid & 12.2 & 12.2 & 17.7 & 2.6 & 1.6 & 14.5 & 1.5 & 6.7 & 19.5 & 11.3 & 16.6 & 5.7 \\
\hline 1,3-Dihydroxy-2-Propanone & 135.8 & 6.6 & 180.8 & 2.4 & 1.2 & 3.0 & 1.2 & 11.7 & 1.2 & 0.5 & 1.5 & 5.1 \\
\hline 2-Phenoxy-Ethanol & 17.7 & 1.1 & 16.9 & 3.9 & 13.1 & 11.8 & 13.9 & 5.3 & 55.8 & 0.5 & 49.5 & 0.9 \\
\hline Isophthalaldehyde & 3.9 & 10.1 & 4.0 & 1.2 & 3.6 & 1.3 & 3.2 & 5.2 & 6.1 & 3.9 & 6.5 & 0.7 \\
\hline Cyclotetradecane & 7.2 & 1.3 & 7.8 & 2.4 & 2.1 & 9.2 & 3.0 & 3.8 & 3.1 & 2.7 & 3.0 & 8.4 \\
\hline Nonanoic Acid & 14.9 & 11.2 & 14.1 & 3.2 & 1.2 & 2.8 & 1.2 & 1.5 & 57.2 & 1.9 & 50.9 & 1.0 \\
\hline 2-Ethyl-Phenol & 32.1 & 12.4 & 36.2 & 0.7 & 22.8 & 0.3 & 26.7 & 13.1 & 11.4 & 4.6 & 13.5 & 4.5 \\
\hline 2-Methoxy-4-Vinyl-Phenol & 77.4 & 6.1 & 83.4 & 2.5 & 64.5 & 0.5 & 69.4 & 4.2 & 4.6 & 0.6 & 4.2 & 2.4 \\
\hline 4-Methylimidazole & 15.3 & 13.6 & 20.4 & 4.7 & 10.8 & 8.2 & 8.8 & 2.7 & 103.4 & 4.4 & 99.9 & 10.6 \\
\hline m-tert-Butyl-Toluene & 11.9 & 1.8 & 14.6 & 2.3 & 11.2 & 2.1 & 9.7 & 2.3 & 16.1 & 1.1 & 16.5 & 2.9 \\
\hline Syringol & 23.3 & 5.4 & 21.3 & 7.0 & 12.3 & 12.1 & 11.9 & 1.5 & 14.1 & 3.9 & 14.0 & 1.9 \\
\hline Decanoic Acid & 29.4 & 6.6 & 31.0 & 4.8 & 1.9 & 0.7 & 1.5 & 1.8 & 32.9 & 8.2 & 35.3 & 12.5 \\
\hline 3-Hydroxy-2,3-Dihydro-Maltol & 19.1 & 0.4 & 24.5 & 6.2 & 1.2 & 10.0 & 1.4 & 0.4 & 4.7 & 5.2 & 5.2 & 12.2 \\
\hline 5-Hydroxy-Maltol & 38.0 & 4.3 & 30.6 & 1.9 & 1.4 & 10.7 & 1.6 & 14.2 & 47.5 & 4.4 & 44.4 & 1.4 \\
\hline Triethylene Glycol & 12.1 & 4.7 & 14.8 & 0.4 & 2.1 & 2.2 & 1.7 & 9.6 & 5.1 & 2.3 & 4.4 & 4.6 \\
\hline Benzoic Acid & 14.3 & 0.6 & 13.6 & 11.2 & 21.2 & 3.3 & 19.6 & 2.3 & 3.3 & 1.8 & 3.1 & 8.5 \\
\hline 2-Methyl-Benzaldehyde & 487.1 & 3.4 & 580.0 & 5.3 & 531.0 & 2.9 & 616.0 & 1.2 & 20.6 & 5.5 & 17.6 & 13.0 \\
\hline Undecanoic Acid & 24.4 & 9.9 & 29.6 & 0.8 & 2.0 & 3.3 & 1.5 & 1.3 & 68.7 & 7.9 & 71.6 & 5.2 \\
\hline 4-Pyridinol & 88.1 & 5.1 & 93.9 & 5.3 & 17.6 & 4.2 & 18.1 & 5.6 & 66.2 & 7.6 & 72.3 & 5.1 \\
\hline 4-Amino-Phenol & 37.6 & 5.0 & 40.3 & 4.2 & 14.0 & 6.4 & 18.0 & 2.2 & 4.7 & 5.8 & 4.0 & 6.6 \\
\hline
\end{tabular}


${ }^{1}$ RSD - Relative Standard Deviation values.

Table S4. Mean, minimum and maximum peak area values of identified VOCs in sugarcane samples.

\begin{tabular}{|c|c|c|c|c|c|c|c|c|c|c|c|c|c|c|c|c|c|c|}
\hline \multirow{3}{*}{$\begin{array}{l}\text { Volatile Organic } \\
\text { Compound }\end{array}$} & \multicolumn{18}{|c|}{ Relative Peak Areas $\left(x 10^{3}\right)$} \\
\hline & \multicolumn{3}{|c|}{ AMA } & \multicolumn{3}{|c|}{ RAD } & \multicolumn{3}{|c|}{ ROX } & \multicolumn{3}{|c|}{ VER } & \multicolumn{3}{|c|}{ VIO } & \multicolumn{3}{|c|}{ CAN } \\
\hline & Min & Max & Mean & Min & Max & Mean & Min & Max & Mean & Min & Max & Mean & Min & Max & Mean & Min & Max & Mean \\
\hline Pentane & 1.7 & 2.5 & 2.1 & 4.1 & 4.7 & 4.3 & 5.1 & 8.3 & 6.9 & 1.9 & 2.7 & 2.4 & 3.0 & 3.7 & 3.3 & 1.9 & 2.3 & 2.2 \\
\hline 1-Pentene & 1.4 & 1.6 & 1.5 & 1.5 & 2.0 & 1.7 & 2.6 & 2.9 & 2.8 & 1.0 & 1.5 & 1.3 & 0.8 & 1.0 & 0.9 & 2.0 & 3.5 & 2.7 \\
\hline 2-Methyl-Pentane & 0.9 & 1.6 & 1.2 & 1.1 & 2.1 & 1.6 & 4.3 & 7.9 & 5.8 & 2.7 & 3.9 & 3.3 & 1.0 & 1.2 & 1.1 & 0.1 & 0.2 & 0.1 \\
\hline Hexane & 1.3 & 2.4 & 1.8 & 1.1 & 1.4 & 1.2 & 3.4 & 4.0 & 3.8 & 1.9 & 2.4 & 2.2 & 1.5 & 1.8 & 1.6 & 10.5 & 21.1 & 15.5 \\
\hline Ethyl Ether & 0.3 & 6.4 & 3.1 & 0.7 & 3.8 & 2.2 & 0.8 & 1.5 & 1.1 & 0.2 & 0.4 & 0.3 & 1.0 & 1.4 & 1.2 & 2.2 & 5.7 & 3.9 \\
\hline cis-4-Methyl-2-Pentene & 0.4 & 0.7 & 0.5 & 0.2 & 1.4 & 0.7 & 0.9 & 4.8 & 2.6 & 0.1 & 2.6 & 1.3 & 0.2 & 0.6 & 0.4 & 0.2 & 0.5 & 0.3 \\
\hline cis-Piperylene & 1.9 & 3.5 & 2.5 & 4.7 & 6.5 & 5.8 & 10.2 & 21.9 & 15.2 & 7.9 & 11.1 & 9.6 & 1.3 & 2.7 & 1.9 & 2.9 & 3.9 & 3.4 \\
\hline 2-Methyl-1-Pentene & 0.9 & 17.5 & 8.8 & 0.5 & 48.9 & 22.5 & 2.2 & 17.3 & 9.5 & 1.3 & 95.7 & 46.0 & 0.9 & 39.6 & 18.2 & 44.9 & 85.0 & 64.2 \\
\hline cis-3-Methyl-2-Pentene & 1.0 & 1.2 & 1.1 & 4.1 & 8.0 & 5.9 & 0.9 & 1.4 & 1.1 & 2.0 & 3.2 & 2.6 & 1.2 & 1.5 & 1.3 & 1.3 & 2.0 & 1.7 \\
\hline 3-Methyl-Hexane & 0.2 & 0.4 & 0.3 & 0.5 & 0.8 & 0.6 & 0.8 & 0.9 & 0.9 & 0.6 & 0.9 & 0.7 & 0.9 & 1.4 & 1.2 & 2.3 & 3.2 & 2.7 \\
\hline 1,4-Pentadiene & 3.1 & 12.3 & 7.2 & 4.2 & 13.3 & 8.6 & 6.0 & 28.1 & 17.1 & 5.8 & 7.7 & 6.5 & 1.7 & 2.1 & 1.9 & 2.3 & 6.1 & 3.9 \\
\hline Methanethiol & 3.1 & 3.2 & 3.2 & 4.0 & 4.9 & 4.4 & 5.9 & 8.1 & 7.0 & 3.3 & 4.5 & 3.8 & 1.9 & 2.5 & 2.1 & 3.0 & 3.9 & 3.5 \\
\hline trans-Piperylene & 2.2 & 4.1 & 3.1 & 1.3 & 2.8 & 2.0 & 3.0 & 5.7 & 4.3 & 1.3 & 1.8 & 1.6 & 0.6 & 0.9 & 0.8 & 1.4 & 2.4 & 1.9 \\
\hline Heptane & 8.5 & 10.6 & 9.3 & 4.9 & 7.1 & 6.1 & 10.2 & 15.9 & 12.9 & 1.3 & 2.3 & 1.8 & 5.3 & 8.7 & 7.0 & 11.2 & 14.7 & 12.6 \\
\hline tert-Butyl Ethyl Ether & 0.1 & 61.6 & 28.9 & 0.4 & 70.4 & 35.3 & 0.4 & 47.9 & 23.9 & 1.2 & 166.6 & 81.0 & 1.0 & 81.6 & 38.5 & 249.7 & 331.7 & 282.0 \\
\hline Ethanal & 53.9 & 77.8 & 63.7 & 20.8 & 39.9 & 30.3 & 30.6 & 46.1 & 38.5 & 65.1 & 72.3 & 68.2 & 10.0 & 18.1 & 14.4 & 55.1 & 68.4 & 62.2 \\
\hline Dimethyl Sulfide & 770.0 & 836.6 & 796.4 & 398.0 & 599.1 & 500.6 & 273.5 & 329.2 & 309.1 & 478.1 & 648.6 & 539.8 & 494.6 & 687.1 & 550.9 & 220.1 & 314.0 & 265.8 \\
\hline 4-Methyl-Heptane & 7.5 & 22.3 & 14.0 & 4.0 & 13.3 & 8.5 & 6.4 & 17.7 & 11.9 & 1.8 & 107.6 & 53.4 & 1.0 & 35.1 & 17.1 & 105.4 & 185.5 & 149.5 \\
\hline Octane & 4.0 & 5.6 & 4.8 & 6.6 & 7.3 & 7.0 & 7.9 & 10.4 & 9.0 & 1.9 & 3.7 & 2.7 & 1.2 & 1.7 & 1.4 & 7.3 & 11.1 & 9.2 \\
\hline Propanal & 8.9 & 10.8 & 9.8 & 3.3 & 8.7 & 5.9 & 5.6 & 11.6 & 8.5 & 5.7 & 8.6 & 7.0 & 3.3 & 5.3 & 4.1 & 16.9 & 28.2 & 21.4 \\
\hline Furan & 8.5 & 11.6 & 10.0 & 6.6 & 10.2 & 8.3 & 7.3 & 10.8 & 8.9 & 7.1 & 8.4 & 7.7 & 4.2 & 5.5 & 5.0 & 4.6 & 6.4 & 5.3 \\
\hline 2,4-Dimethyl-Heptane & 11.5 & 24.5 & 17.3 & 16.2 & 19.8 & 18.0 & 24.4 & 30.1 & 27.4 & 17.6 & 22.0 & 19.3 & 13.4 & 22.6 & 17.5 & 11.2 & 13.3 & 11.9 \\
\hline
\end{tabular}




\begin{tabular}{|c|c|c|c|c|c|c|c|c|c|c|c|c|c|c|c|c|c|c|}
\hline 2-Methyl-Propanal & 20.4 & 24.9 & 22.6 & 38.3 & 42.8 & 39.9 & 49.6 & 75.7 & 64.3 & 80.4 & 91.8 & 87.0 & 20.9 & 22.1 & 21.7 & 26.1 & 29.4 & 27.6 \\
\hline 2-Propanone & 242.3 & 321.5 & 295.2 & 405.3 & 496.9 & 449.0 & 75.9 & 96.3 & 85.7 & 432.6 & 496.9 & 465.6 & 66.3 & 177.8 & 122.8 & 626.3 & 786.0 & 701.1 \\
\hline Methyl Acetate & 2.2 & 4.2 & 3.1 & 0.8 & 1.6 & 1.2 & 2.1 & 32.7 & 16.2 & 4.8 & 7.5 & 6.0 & 0.8 & 1.7 & 1.2 & 0.3 & 0.5 & 0.4 \\
\hline 2-Methyl-2-Heptene & 0.4 & 0.6 & 0.5 & 1.1 & 2.0 & 1.6 & 1.9 & 3.3 & 2.6 & 0.3 & 0.8 & 0.6 & 0.9 & 0.9 & 0.9 & 23.6 & 35.4 & 29.9 \\
\hline Crotonal & 0.1 & 0.3 & 0.2 & 2.5 & 3.4 & 2.8 & 4.2 & 5.0 & 4.5 & 4.2 & 5.4 & 4.8 & 3.3 & 7.9 & 5.5 & 1.7 & 1.9 & 1.8 \\
\hline 2,3-Dimethyl-Heptane & 1.1 & 3.1 & 2.0 & 1.5 & 2.5 & 1.9 & 2.2 & 6.0 & 3.9 & 2.0 & 3.2 & 2.5 & 0.7 & 0.9 & 0.8 & 0.3 & 0.7 & 0.5 \\
\hline 4-Methyl-Octane & 8.6 & 11.8 & 9.7 & 7.6 & 9.3 & 8.2 & 17.4 & 22.1 & 20.3 & 8.6 & 10.6 & 9.7 & 8.4 & 11.6 & 9.8 & 5.3 & 7.0 & 6.2 \\
\hline 2-Methyl-Furan & 1.6 & 2.2 & 1.8 & 2.7 & 3.9 & 3.2 & 1.0 & 1.4 & 1.2 & 1.0 & 1.9 & 1.4 & 1.2 & 2.0 & 1.7 & 1.1 & 1.8 & 1.4 \\
\hline 4-Dimethyl-1-Heptene & 5.1 & 7.9 & 6.6 & 6.5 & 8.7 & 7.7 & 17.2 & 30.5 & 24.5 & 9.5 & 12.3 & 11.2 & 10.6 & 28.8 & 18.9 & 314.7 & 432.6 & 386.2 \\
\hline Ethyl Acetate & 21.5 & 52.8 & 36.5 & 15.3 & 23.7 & 18.9 & 435.1 & 603.7 & 499.8 & 61.2 & 76.7 & 68.4 & 9.1 & 18.4 & 13.2 & 42.8 & 65.4 & 55.3 \\
\hline Nonane & 1.3 & 1.7 & 1.5 & 2.4 & 4.6 & 3.4 & 4.7 & 5.6 & 5.2 & 1.5 & 2.7 & 2.1 & 2.6 & 4.1 & 3.1 & 4.4 & 6.6 & 5.5 \\
\hline 2-Butanone & 8.8 & 14.0 & 11.1 & 3.8 & 5.7 & 4.6 & 11.2 & 19.0 & 15.4 & 15.5 & 21.6 & 18.5 & 6.2 & 6.7 & 6.4 & 29.4 & 33.4 & 31.9 \\
\hline 2-Methyl-Butanal & 86.2 & 117.1 & 99.8 & 59.4 & 71.1 & 65.9 & 45.7 & 67.2 & 57.9 & 79.6 & 103.8 & 89.5 & 12.9 & 16.4 & 14.6 & 64.5 & 76.1 & 69.3 \\
\hline 3-Methyl-Butanal & 76.0 & 131.9 & 96.9 & 49.5 & 59.7 & 54.5 & 39.9 & 60.4 & 51.3 & 82.0 & 94.7 & 86.9 & 13.2 & 27.4 & 19.4 & 65.5 & 94.7 & 78.7 \\
\hline Ethyl Alcohol & 427.8 & 562.6 & 485.2 & 837.1 & 989.4 & 924.1 & 723.0 & 928.0 & 802.8 & 533.8 & 683.8 & 609.2 & 887.9 & 1130.3 & 978.3 & 176.6 & 198.9 & 189.9 \\
\hline Benzene & 1.4 & 2.4 & 1.9 & 8.1 & 10.3 & 9.2 & 10.5 & 20.0 & 14.6 & 9.8 & 13.8 & 11.5 & 5.1 & 9.0 & 7.1 & 5.1 & 5.9 & 5.4 \\
\hline 3-Ethyl-Heptane & 2.3 & 3.0 & 2.8 & 1.5 & 1.7 & 1.6 & 2.8 & 3.7 & 3.2 & 0.6 & 0.8 & 0.7 & 0.9 & 2.0 & 1.4 & 5.9 & 8.6 & 7.2 \\
\hline 2-Ethyl-Furan & 9.8 & 14.4 & 12.7 & 19.3 & 32.4 & 26.4 & 21.8 & 29.6 & 25.0 & 15.9 & 39.1 & 27.4 & 17.4 & 22.8 & 19.4 & 15.6 & 22.8 & 18.6 \\
\hline Ethyl Propanoate & 0.3 & 0.6 & 0.4 & 7.5 & 9.1 & 8.3 & 57.3 & 100.4 & 76.3 & 1.3 & 2.1 & 1.6 & 0.2 & 0.2 & 0.2 & 0.7 & 1.2 & 0.9 \\
\hline thyl 2-Methylpropanoate & 0.3 & 0.4 & 0.3 & 0.2 & 0.2 & 0.2 & 20.1 & 31.0 & 25.6 & 0.2 & 0.2 & 0.2 & 0.1 & 0.2 & 0.1 & 0.2 & 0.3 & 0.2 \\
\hline 2-Methyl-Crotonal & 1.3 & 2.3 & 1.8 & 0.8 & 0.8 & 0.8 & 5.6 & 5.8 & 5.7 & 6.1 & 7.4 & 6.6 & 2.4 & 3.1 & 2.8 & 1.0 & 1.3 & 1.2 \\
\hline Propyl Acetate & 0.7 & 0.9 & 0.8 & 0.8 & 1.0 & 0.9 & 10.0 & 17.2 & 13.3 & 0.7 & 0.9 & 0.8 & 0.0 & 0.1 & 0.1 & 0.3 & 0.4 & 0.3 \\
\hline 3-Pentanone & 56.4 & 90.9 & 70.4 & 92.0 & 107.0 & 97.0 & 85.9 & 109.1 & 95.4 & 97.2 & 127.4 & 116.0 & 53.7 & 66.4 & 62.0 & 134.3 & 179.0 & 151.7 \\
\hline Pentanal & 0.4 & 0.5 & 0.4 & 0.2 & 0.3 & 0.2 & 2.1 & 4.0 & 3.1 & 1.0 & 1.4 & 1.2 & 0.9 & 1.1 & 1.0 & 11.4 & 15.5 & 13.1 \\
\hline 2,3-Butanedione & 21.8 & 25.4 & 23.5 & 38.7 & 44.4 & 41.2 & 34.0 & 42.7 & 39.0 & 37.8 & 41.1 & 39.2 & 26.8 & 33.7 & 28.8 & 13.0 & 18.0 & 15.1 \\
\hline 2,3,4-Trimethyl-Hexane & 0.3 & 0.4 & 0.4 & 0.5 & 0.7 & 0.6 & 0.8 & 1.7 & 1.2 & 0.1 & 0.2 & 0.1 & 0.5 & 0.6 & 0.5 & 9.8 & 14.1 & 12.5 \\
\hline Decane & 0.4 & 0.5 & 0.4 & 0.2 & 0.2 & 0.2 & 3.0 & 3.9 & 3.4 & 1.9 & 2.2 & 2.1 & 4.3 & 5.3 & 4.9 & 12.3 & 25.8 & 18.9 \\
\hline 2,6-Dimethyl-Nonane & 1.6 & 2.3 & 1.9 & 0.9 & 1.4 & 1.1 & 2.4 & 2.9 & 2.7 & 0.6 & 0.8 & 0.7 & 0.8 & 1.4 & 1.1 & 12.9 & 22.7 & 18.4 \\
\hline 2,5-Dimethyl-Nonane & 0.4 & 0.6 & 0.5 & 0.9 & 1.5 & 1.2 & 6.0 & 8.0 & 6.8 & 0.9 & 1.4 & 1.1 & 1.2 & 1.9 & 1.6 & 0.3 & 0.3 & 0.3 \\
\hline 4-Methyl-2-Pentanone & 1.0 & 1.2 & 1.1 & 0.2 & 0.2 & 0.2 & 0.2 & 0.5 & 0.3 & 0.9 & 1.2 & 1.1 & 0.4 & 0.7 & 0.5 & 19.9 & 30.4 & 25.3 \\
\hline
\end{tabular}


2-Methy-2-Butanol

2-Methylpropyl Acetate

$$
\alpha \text {-Pinene }
$$

4,4-Dimethyl-2-

Pentanone

1-Penten-3-one

5-Butyl-Nonane

3,6-Dimethyl-Decane

2-Methyl-3-Buten-2-ol

5-Methyl-Decane

4-Methyl-Decane

2,3-Pentanedione

Methyl 3,3-

Dimethylbutanoate

$$
\text { 2-Hexanone }
$$

4-Methyl-Undecane Hexanal

2-Methyl-1-Propanol

2-Methyl-2-Pentanol

Ethyl-Benzene

3-Methylbutyl Acetate

$$
\text { o-Xylene }
$$

2-Pentanol

$$
\text { p-Xylene }
$$

5-Methyl-2-Hexanone

Methyl 2-Ethylpentanoate

$$
\text { 1-Butanol }
$$

$\alpha$-Phellandrene

2-Methyl-3-Pentanol

1-Penten-3-ol

$\alpha$-Terpinene
0.4

0.4

0.2

0.2

$0.2 \quad 0.4$

1.8
0.

0.1

0.1

0.3

$$
2.9
$$

0.2

0.4

1.0

1.0

11.921.

0.8

0.5

$\begin{array}{ll}0.6 & 0.8\end{array}$

$\begin{array}{ll}6.7 \quad 8.8 \\ 36.2 & 47.0\end{array}$

$36.2 \quad 47.0$

0.30 .5

$28.0 \quad 36.5$

$\begin{array}{ll}7.8 & 10.9\end{array}$

$11.9 \quad 21.3$

$0.3 \quad 0.5$

$6.6 \quad 8.9$

$0.6 \quad 0.7$

$0.3 \quad 0.6$

$\begin{array}{ll}0.6 & 0.7\end{array}$

$0.1 \quad 0.2$

$\begin{array}{ll}4.0 & 6.4\end{array}$

$57.4 \quad 73.7$

$0.4 \quad 0.5$
0

$$
0.1
$$$$
0.4
$$

3.5

$$
0.3
$$

$$
0.6
$$$$
1.4
$$$$
1.8
$$$$
0.9
$$

0.6

0.7

7.8

39.5

$0.4 \quad 0.2$

$31.4 \quad 35$

$8.8 \quad 18.2$

$16.1 \quad 8.6$

$0.4 \quad 3.3$

$7.5 \quad 10$

0.7

$0.5 \quad 14.8$

$0.7 \quad 1.3$

0.10 .7

$5.1 \quad 2$.

66.199 .7

$\begin{array}{cc}66.1 & 99.7 \\ 0.5 & 0.1\end{array}$

0.6

1.9
0.2

0.2

1.4

$\begin{array}{lll}0.3 & 0.3 & 4.2\end{array}$

2.1

0.9

2.6

1.1

1.0

$\begin{array}{ll}1.9 & 7.7\end{array}$

$\begin{array}{ll}0.8 & 0.4\end{array}$

2.2

$$
1.0
$$

1.0
0.8
1.2

9.7

1.0

1.8

2.2

12.6

0.8

2.3
0.7

1.2

0.6

0.5

13.7

$26.4 \quad 24.4$

0.2

$0.2 \quad 0.3$

26.5

$40.5 \quad 72.6$

$22.6 \quad 18.2$

$\begin{array}{lll}11.0 & 9.4 & 20.8\end{array}$

28.8

$\begin{array}{ll}4.4 & 63.8 \\ 18.4 & 41.4\end{array}$

0.4

0.32 .8

0.8

165

3.7
165.5
0.2

$\begin{array}{cc}3.1 & 26.3 \\ 130.0 & 141.5\end{array}$

$\begin{array}{lll}0.5 & 0.4 & 0.1\end{array}$

19.2

2.2

$$
\begin{array}{lllll}
0.4 & 0.3 & 0.2 & 0.2 & 0.2 \\
5.2 & 4.5 & 1.7 & 2.2 & 2.0
\end{array}
$$

$9.6 \quad 8.5$

$0.7 \quad 0.5$

$19.6 \quad 14.9$

$1.4 \quad 1.2$

$2.8 \quad 2.1$

$4.1 \quad 3.1$

27.5

$2.5 \quad 1.9$

$\begin{array}{lll}0.5 & 0.6 & 0.6\end{array}$

$\begin{array}{lll}0.1 & 0.2 & 0.1\end{array}$

0.6

0.8

0.2

0.7

1.2

0.3

0.7

1.0

0.3

$\begin{array}{lll}8.1 & 10.5 & 9.3\end{array}$

$\begin{array}{lll}4.1 & 5.4 & 4.8\end{array}$

0.3

0.3

0.3

$\begin{array}{lll}0.5 & 0.8 & 0.7\end{array}$

47.9

45.2

0.9

1.9

1.4

1.4
29.5

20.4

$0.4 \quad 0.3$

$105.6 \quad 87.1$

$15.5 \quad 24.4 \quad 19.2$

$\begin{array}{ccc}15.5 & 24.4 & 19.2 \\ 0.1 & 0.2 & 0.2\end{array}$

$25.7 \quad 22.6$

24.3

10.4

30.6

$33.0 \quad 27.2$

$94.3 \quad 80.1$

9.6
7.7

13.9

17.7

27.5

12.2

$49.3 \quad 45.9$

35.1

8.4
41.7

8.0

$3.5 \quad 3.2$

0.3

0.4
12.0

37.5

0.3

0.5

10.2
0.4

6.8

$\begin{array}{llll}1.8 & 12.3 & 18.9 & 15.5\end{array}$

$\begin{array}{llll}1.8 & 12.3 & 18.9 & 15.5 \\ 0.8 & 0.4 & 0.4 & 0.4\end{array}$

$5.5 \quad 7.9$

$\begin{array}{llll}33.5 & 28.9 & 9.0 & 13.8\end{array}$

$\begin{array}{llll}33.5 & 188.0 & 37.4 & 49.3\end{array}$

11.0

41.9

6.4

$\begin{array}{cccccc}130.0 & 141.5 & 233.5 & 188.0 & 37.4 & 49.3 \\ 0.1 & 0.1 & 0.2 & 0.1 & 4.8 & 7.8\end{array}$ 


\begin{tabular}{|c|c|c|c|c|c|c|c|c|c|c|c|c|c|c|c|c|c|c|}
\hline Butyl Prop-2-enoate & 4.3 & 6.5 & 5.3 & 1.4 & 4.9 & 3.1 & 10.0 & 20.0 & 14.7 & 3.5 & 5.1 & 4.4 & 4.5 & 7.2 & 5.5 & 8.4 & 17.5 & 12.9 \\
\hline m-Xylene & 1.5 & 1.9 & 1.7 & 13.6 & 16.5 & 15.1 & 30.5 & 41.5 & 36.1 & 1.4 & 2.1 & 1.7 & 12.0 & 16.5 & 14.3 & 21.7 & 31.4 & 26.9 \\
\hline 2-Heptanone & 124.9 & 187.9 & 154.5 & 731.2 & 797.7 & 763.0 & 78.5 & 116.9 & 97.4 & 139.7 & 256.6 & 185.9 & 489.0 & 626.0 & 562.5 & 268.0 & 356.7 & 329.0 \\
\hline Heptanal & 0.3 & 0.5 & 0.4 & 9.1 & 11.2 & 10.2 & 4.8 & 8.7 & 6.6 & 0.8 & 0.9 & 0.8 & 1.2 & 1.7 & 1.5 & 4.0 & 5.5 & 4.5 \\
\hline D-Limonene & 0.1 & 0.2 & 0.2 & 0.2 & 0.2 & 0.2 & 2.6 & 3.2 & 2.9 & 0.1 & 0.2 & 0.2 & 1.2 & 1.8 & 1.5 & 1.2 & 2.0 & 1.5 \\
\hline 3-Hexanol & 0.2 & 0.3 & 0.3 & 1.7 & 2.2 & 1.9 & 0.4 & 0.6 & 0.5 & 3.1 & 3.8 & 3.3 & 1.6 & 2.0 & 1.8 & 0.5 & 0.8 & 0.6 \\
\hline$\beta$-Phellandrene & 0.2 & 0.3 & 0.2 & 1.4 & 1.7 & 1.5 & 0.3 & 0.4 & 0.4 & 2.0 & 4.5 & 3.2 & 8.9 & 11.0 & 9.6 & 300.7 & 417.0 & 354.8 \\
\hline Eucalyptol & 0.2 & 0.2 & 0.2 & 0.1 & 0.2 & 0.1 & 0.1 & 0.2 & 0.1 & 0.3 & 0.4 & 0.4 & 0.1 & 0.2 & 0.1 & 0.5 & 0.8 & 0.7 \\
\hline m-Menthane & 0.1 & 0.1 & 0.1 & 0.2 & 0.2 & 0.2 & 1.5 & 2.8 & 2.1 & 0.3 & 0.4 & 0.4 & 0.5 & 0.7 & 0.6 & 2.0 & 2.6 & 2.4 \\
\hline 4-Methyl-2-Heptanone & 0.3 & 0.3 & 0.3 & 22.6 & 31.4 & 26.0 & 2.1 & 4.1 & 3.1 & 2.1 & 2.6 & 2.3 & 1.5 & 2.0 & 1.8 & 366.4 & 452.6 & 423.2 \\
\hline 3-Methyl-2-Pentanol & 11.2 & 20.8 & 14.9 & 79.4 & 100.0 & 89.3 & 82.1 & 89.3 & 85.2 & 4.9 & 7.3 & 6.1 & 84.7 & 101.1 & 91.3 & 1.7 & 3.9 & 2.8 \\
\hline 2-Methyl-1-Butanol & 151.8 & 250.8 & 196.0 & 179.2 & 201.1 & 191.7 & 131.3 & 263.3 & 184.0 & 79.9 & 127.9 & 101.9 & 125.9 & 185.9 & 160.9 & 16.5 & 26.4 & 21.8 \\
\hline 3-Methyl-1-Butanol & 439.5 & 668.2 & 532.4 & 241.6 & 437.0 & 339.9 & 447.9 & 564.4 & 502.7 & 263.0 & 446.1 & 348.1 & 310.8 & 596.6 & 439.6 & 28.7 & 37.2 & 33.7 \\
\hline o-Ethyltoluene & 1.3 & 1.9 & 1.7 & 3.8 & 5.9 & 4.9 & 7.7 & 8.5 & 8.3 & 5.2 & 6.8 & 5.9 & 2.0 & 4.0 & 2.9 & 5.9 & 6.9 & 6.4 \\
\hline m-Ethyltoluene & 1.0 & 2.3 & 1.6 & 3.5 & 4.0 & 3.7 & 13.0 & 15.9 & 14.1 & 6.7 & 9.9 & 8.3 & 6.0 & 7.3 & 6.6 & 1.9 & 3.0 & 2.4 \\
\hline 2-Hexanol & 57.7 & 83.0 & 68.6 & 48.1 & 70.1 & 56.6 & 1.3 & 3.1 & 2.3 & 1.5 & 3.1 & 2.4 & 5.5 & 9.0 & 7.1 & 1.8 & 2.0 & 1.8 \\
\hline $\begin{array}{l}\text { Ethylene Glycol Ethyl } \\
\text { Ether }\end{array}$ & 0.1 & 0.2 & 0.1 & 1.2 & 1.8 & 1.5 & 2.5 & 2.9 & 2.7 & 9.1 & 20.0 & 14.1 & 1.7 & 2.7 & 2.1 & 1.3 & 2.6 & 1.8 \\
\hline 6-Methyl-2-Heptanone & 1.6 & 3.0 & 2.2 & 1.5 & 2.3 & 1.9 & 2.2 & 3.7 & 2.9 & 1.7 & 2.0 & 1.9 & 1.4 & 2.1 & 1.7 & 6.9 & 9.3 & 7.8 \\
\hline Pseudocumene & 2.1 & 3.3 & 2.7 & 3.8 & 6.0 & 4.9 & 7.2 & 8.1 & 7.7 & 4.3 & 5.3 & 4.8 & 2.1 & 4.1 & 3.0 & 4.5 & 5.7 & 5.0 \\
\hline $\begin{array}{l}\text { 4,6-Dimethyl-2- } \\
\text { Heptanone }\end{array}$ & 0.7 & 0.9 & 0.8 & 0.7 & 1.0 & 0.9 & 1.1 & 1.7 & 1.4 & 0.7 & 0 & 0.8 & 0.3 & 0.4 & 0.4 & 39.1 & 61.2 & 51.8 \\
\hline 2,3-Dimethyl-2-Hexanol & 0.2 & 0.4 & 0.3 & 0.2 & 0.3 & 0.2 & 1.0 & 2.4 & 1.6 & 0.2 & 0.3 & 0.2 & 0.2 & 0.4 & 0.3 & 18.3 & 21.5 & 19.6 \\
\hline 3-Octanone & 5.1 & 6.8 & 5.9 & 15.5 & 23.4 & 19.2 & 17.3 & 24.0 & 21.1 & 0.8 & 1.0 & 0.9 & 17.0 & 21.6 & 19.3 & 0.3 & 0.4 & 0.3 \\
\hline Styrene & 26.2 & 45.3 & 35.3 & 31.5 & 35.5 & 33.5 & 81.2 & 132.2 & 101.9 & 47.4 & 64.8 & 56.3 & 48.1 & 65.6 & 53.5 & 23.0 & 30.6 & 27.3 \\
\hline 5-Methyl-2-Heptanone & 4.2 & 6.8 & 5.4 & 9.7 & 18.4 & 13.9 & 7.7 & 9.6 & 8.7 & 9.1 & 10.6 & 9.8 & 7.4 & 8.6 & 7.7 & 16.9 & 26.5 & 22.4 \\
\hline 1-Pentanol & 30.0 & 52.0 & 39.6 & 27.4 & 34.2 & 30.2 & 3.9 & 4.9 & 4.5 & 27.2 & 48.2 & 38.0 & 6.3 & 8.9 & 7.7 & 4.7 & 6.0 & 5.3 \\
\hline p-Ethyltoluene & 0.2 & 0.4 & 0.3 & 2.2 & 3.3 & 2.7 & 6.6 & 9.2 & 7.5 & 4.4 & 5.5 & 4.9 & 3.0 & 4.6 & 3.8 & 1.8 & 2.7 & 2.2 \\
\hline p-Cymene & 1.4 & 2.1 & 1.7 & 2.2 & 2.5 & 2.3 & 3.5 & 4.0 & 3.8 & 11.4 & 18.1 & 14.5 & 3.2 & 3.7 & 3.5 & 30.9 & 52.2 & 39.5 \\
\hline Mesitylene & 8.4 & 13.2 & 10.5 & 1.2 & 1.3 & 1.2 & 0.4 & 0.5 & 0.4 & 8.2 & 11.8 & 10.2 & 14.0 & 15.2 & 14.7 & 8.0 & 11.2 & 9.1 \\
\hline 4-Methyl-4-Heptanol & 1.0 & 1.8 & 1.4 & 0.5 & 0.6 & 0.5 & 0.1 & 0.2 & 0.2 & 0.4 & 0.5 & 0.4 & 0.2 & 0.4 & 0.3 & 0.7 & 1.1 & 0.8 \\
\hline
\end{tabular}




\begin{tabular}{|c|c|c|c|c|c|c|c|c|c|c|c|c|c|c|c|c|c|c|}
\hline 4-Heptanol & 0.4 & 0.5 & 0.4 & 2.4 & 3.0 & 2.6 & 2.8 & 3.6 & 3.2 & 3.3 & 3.9 & 3.7 & 1.4 & 2.1 & 1.7 & 10.5 & 19.7 & 14.0 \\
\hline 2-Octanone & 21.9 & 39.4 & 30.0 & 98.8 & 117.5 & 107.3 & 11.8 & 21.1 & 16.8 & 27.7 & 43.8 & 34.5 & 67.1 & 84.0 & 77.5 & 65.4 & 96.1 & 81.6 \\
\hline Octanal & 0.2 & 0.2 & 0.2 & 0.1 & 0.2 & 0.1 & 4.1 & 4.6 & 4.3 & 1.4 & 2.3 & 1.8 & 1.5 & 1.9 & 1.7 & 3.8 & 4.7 & 4.2 \\
\hline 3-Hydroxy-2-Butanone & 0.2 & 0.3 & 0.2 & 0.3 & 0.5 & 0.4 & 0.1 & 0.1 & 0.1 & 0.2 & 0.3 & 0.2 & 0.2 & 0.3 & 0.2 & 0.1 & 0.1 & 0.1 \\
\hline 1-Octen-3-one & 15.1 & 23.6 & 18.8 & 6.2 & 7.5 & 6.9 & 2.6 & 3.3 & 3.0 & 3.4 & 4.4 & 3.8 & 0.2 & 0.3 & 0.2 & 14.9 & 23.7 & 19.3 \\
\hline trans-2-Pentenol & 10.4 & 13.4 & 11.8 & 21.7 & 24.0 & 23.2 & 22.5 & 26.2 & 23.9 & 15.6 & 26.0 & 19.6 & 14.4 & 22.7 & 18.6 & 13.7 & 17.6 & 16.0 \\
\hline $\begin{array}{l}\text { 2,2,6-Trimethyl- } \\
\text { Cyclohexanone }\end{array}$ & 2.0 & 2.6 & 2.3 & 2.9 & 3.5 & 3.1 & 4.7 & 5.7 & 5.1 & 1.9 & 2.3 & 2.1 & 2.5 & 3.6 & 3.0 & 3.6 & 4.1 & 3.8 \\
\hline 2-Heptanol & 503.2 & 589.8 & 553.6 & 484.5 & 631.5 & 549.0 & 260.5 & 289.9 & 273.6 & 561.6 & 665.8 & 627.5 & 595.9 & 760.8 & 692.6 & 372.6 & 475.0 & 423.5 \\
\hline cis-2-Pentenol & 206.9 & 331.8 & 257.8 & 119.8 & 201.6 & 164.7 & 141.0 & 155.8 & 148.0 & 209.3 & 315.4 & 251.4 & 93.2 & 147.2 & 113.7 & 121.8 & 155.2 & 137.8 \\
\hline 1-Hydroxy-2-Propanone & 20.3 & 24.7 & 22.0 & 8.9 & 11.9 & 10.1 & 5.2 & 7.1 & 6.1 & 87.3 & 126.9 & 105.8 & 4.3 & 6.1 & 5.3 & 3.6 & 4.9 & 4.3 \\
\hline 5-Methyl-4-Hexen-3-one & 1.2 & 1.6 & 1.4 & 2.0 & 3.5 & 2.7 & 1.3 & 2.1 & 1.8 & 2.2 & 2.8 & 2.4 & 2.9 & 3.4 & 3.2 & 1.6 & 2.1 & 1.8 \\
\hline Hemimellitene & 4.0 & 5.3 & 4.5 & 2.2 & 3.8 & 2.9 & 7.0 & 9.5 & 8.5 & 5.3 & 5.9 & 5.5 & 3.4 & 4.7 & 4.1 & 2.5 & 4.4 & 3.2 \\
\hline 6-Methyl-5-Hepten-2-one & 14.1 & 21.7 & 17.4 & 9.1 & 13.3 & 11.3 & 17.1 & 25.7 & 21.1 & 10.0 & 17.0 & 12.9 & 9.2 & 14.5 & 11.4 & 40.3 & 45.9 & 42.5 \\
\hline 2-Pentene & 272.3 & 363.4 & 306.5 & 400.7 & 459.4 & 439.3 & 92.8 & 148.2 & 119.8 & 294.2 & 457.2 & 395.5 & 475.8 & 506.7 & 486.2 & 308.2 & 435.5 & 388.6 \\
\hline 1-Hexanol & 527.7 & 721.0 & 610.1 & 366.0 & 424.6 & 402.9 & 630.0 & 717.2 & 680.2 & 633.3 & 746.6 & 696.4 & 359.4 & 472.9 & 411.8 & 102.3 & 192.7 & 153.5 \\
\hline 2-Cyclopenten-1-one & 0.9 & 1.3 & 1.1 & 9.6 & 12.6 & 10.6 & 7.7 & 15.8 & 11.5 & 13.3 & 16.7 & 15.0 & 1.4 & 2.1 & 1.7 & 8.1 & 15.1 & 11.5 \\
\hline $\begin{array}{l}\text { 2-Methyl-2-Cyclopenten- } \\
\text { 1-one }\end{array}$ & 0.2 & 0.3 & 0.3 & 3.0 & 3 & 3.4 & 2.8 & 3. & 3.1 & 3 & 3.9 & 3.5 & 0.5 & 6.4 & 3.3 & 4.0 & 5.2 & 6 \\
\hline$\beta$-Methy-Styrene & 0.2 & 0.4 & 0.3 & 1.7 & 2.1 & 1.9 & 3.1 & 4.1 & 3.7 & 3.3 & 3.5 & 3.4 & 2.3 & 3.2 & 2.6 & 0.7 & 0.8 & 0.8 \\
\hline 4-Octanol & 3.4 & 3.9 & 3.6 & 3.4 & 4.0 & 3.8 & 1.6 & 2.4 & 2.0 & 3.1 & 4.0 & 3.7 & 3.2 & 4.4 & 3.9 & 5.2 & 6.6 & 5.8 \\
\hline cis-3-Hexen-1-ol & 9.8 & 16.5 & 13.1 & 6.8 & 9.0 & 7.8 & 8.5 & 11.1 & 10.2 & 5.2 & 7.3 & 6.3 & 4.3 & 5.2 & 4.7 & 1.5 & 1.7 & 1.6 \\
\hline 2-Nonanone & 46.5 & 59.6 & 52.8 & 296.6 & 385.7 & 350.6 & 52.3 & 69.4 & 60.7 & 12.5 & 21.6 & 16.4 & 143.4 & 276.5 & 204.1 & 1.2 & 1.4 & 1.3 \\
\hline Nonanal & 80.8 & 93.4 & 85.5 & 77.3 & 88.0 & 83.0 & 11.9 & 26.2 & 19.1 & 37.4 & 51.7 & 43.5 & 36.4 & 51.3 & 42.8 & 2.8 & 4.2 & 3.7 \\
\hline $\begin{array}{c}\text { Ethylene Glycol Butyl } \\
\text { Ether }\end{array}$ & 25.6 & 28.7 & 27.5 & 2.4 & 2.9 & 2.7 & 9.9 & 17.3 & 12.7 & 21.8 & 24.0 & 23.1 & 5.1 & 5.9 & 5.4 & 1.2 & 1.8 & 1.5 \\
\hline $\begin{array}{l}\text { 1-Ethyl-1-Methyl- } \\
\text { Cyclopentane }\end{array}$ & 1.5 & 2.3 & 1.9 & 2.9 & 4.0 & 3.5 & 11.1 & 18.5 & 15.0 & 6.1 & 7.7 & 6.9 & 3.4 & 4.7 & 4.3 & 49.4 & 70.6 & 58.2 \\
\hline 3-Octen-2-one & 2.5 & 3.2 & 2.8 & 82.2 & 171.8 & 124.7 & 6.6 & 7.8 & 7.2 & 7.7 & 9.0 & 8.3 & 2.1 & 3.1 & 2.7 & 28.6 & 38.5 & 33.3 \\
\hline 2-Octanol & 77.8 & 110.0 & 88.6 & 142.1 & 175.6 & 162.6 & 10.3 & 10.9 & 10.6 & 82.3 & 113.5 & 97.3 & 241.8 & 308.3 & 274.6 & 45.0 & 59.7 & 53.8 \\
\hline $\begin{array}{c}\text { 3-Ethyl-2-Methyl-1,3- } \\
\text { Hexadiene }\end{array}$ & 0.3 & 0.3 & 0.3 & 0.2 & 0.3 & 0.3 & 0.9 & 1.2 & 1.0 & 0.3 & 0.4 & 0.3 & 2.4 & 2.7 & 2.5 & 0.2 & 0.3 & 0.2 \\
\hline
\end{tabular}


1,3-Bis (1,1-

Dimethylethyl)-Benzene

m-Ethyl-Styrene

$\begin{array}{llll}0.3 & 0.4 & 0.4 & 0.2\end{array}$

-Methyl-2-Heptanone

2-Decanone

$0.8 \quad 8.3$

$\begin{array}{lll}0.2 & 0.2 & 0.2\end{array}$

$0.2 \quad 0.2$

0.1

0.2

0.2

0.4

0.5

.

$\begin{array}{llll}9.2 & 11.4 & 10.2 & 8.3\end{array}$

0.3

17.0

$23.4 \quad 20.0$

1.4

0.5

1.9

9.7

4.0

1-Octen-3-ol

$0.7 \quad 0.5$

$0.6 \quad 0.5$

0.7

$0.9 \quad 0.8$

$\begin{array}{lll}0.7 & 0.8 & 0.7\end{array}$

$\begin{array}{ll}0.4 & 0.6 \\ 6.5 & 8.4\end{array}$

$3.1 \quad 12.2$

$106.6 \quad 148.8$

125.898

2.8
135.5

$\begin{array}{llll}2.3 & 1.0 & 1.8 & 1.5\end{array}$

$\begin{array}{lll}1.5 & 1.8 & 1.7\end{array}$

trans,trans-2,4-
Heptadienal

1-Heptanol

$1.8 \quad 2$

$48.1 \quad 58.9$

cis,cis-3,5-Octadiene

$\begin{array}{lll}1.6 & 2.6 & 52.9\end{array}$

Methyl 2-Oxopropanoate

Dihydromyrcenol

$\begin{array}{llll}130.3 & 187.5 & 157.1 & 90.8\end{array}$

trans-Menthone

Furfural

Ethanoic Acid

0.1

2.6

$\begin{array}{lll}59.4 & 82.0 & 70.7\end{array}$

$\begin{array}{llll}1861.2 & 2563.4 & 2073.7 & 1799.1\end{array}$

2-Ethyl-1-Hexanol

Decanal$$
30.1 \quad 38.3 \quad 33.1 \quad 12.8
$$

$24.3 \quad 33.9-29.3$

$46.8 \quad 58.5 \quad 52.2$

Furfuryl Formate

$6.8 \quad 58.5$

22.2

trans-2-Hepten-1-ol

$\begin{array}{lll}19.6 & 25.3 \quad 22.6\end{array}$

$\begin{array}{lll}299.6 & 400.0 \quad 335.8\end{array}$

35.8432

1-(2-Furanyl)-Ethanone

3,5-Octadien-2-one

Benzaldehyde

Furfuryl Acetate

Linalool

$\beta$-Myrcene

1-Octanol

trans-p-2-Menthen-1-ol

Propanoic Acid

2,3-Butanediol

11.7

$\begin{array}{lllllll}28.4 & 35.3 & 30.5 & 21.4 & 28.1 & 25.5 & 33.3\end{array}$

$\begin{array}{lllllll}0.4 & 0.4 & 0.4 & 0.3 & 0.4 & 0.4 & 0.7\end{array}$

$6.4 \quad 7.9$

$0.8 \quad 1.0$

$7.0 \quad 4.7$

$80.4 \quad 101.1 \quad 88.2 \quad 64$.

$\begin{array}{cccccc}0.1 & 0.1 & 0.1 & 0.7 & 0.9 & 0.8 \\ 4.0 & 4.9 & 4.4 & 0.8 & 1.0 & 0.9 \\ 230.3 & 361.6 & 284.6 & 415.1 & 459.8 & 441.4\end{array}$

$\begin{array}{lllllll}230.3 & 361.6 & 284.6 & 415.1 & 459.8 & 441.4 & 51.5\end{array}$ 


\begin{tabular}{|c|c|c|c|c|c|c|c|c|c|c|c|c|c|c|c|c|c|c|}
\hline Dimethyl Sulfoxide & 59.1 & 73.7 & 65.4 & 30.2 & 36.2 & 33.6 & 18.8 & 26.4 & 22.4 & 99.8 & 107.2 & 101.8 & 49.2 & 73.3 & 59.9 & 4.7 & 5.8 & 5.4 \\
\hline m-Menth-3-ene & 48.7 & 62.0 & 54.0 & 43.6 & 57.4 & 49.7 & 6.2 & 8.3 & 7.1 & 31.4 & 48.4 & 38.7 & 11.7 & 14.8 & 13.3 & 6.0 & 7.1 & 6.6 \\
\hline $\begin{array}{l}\text { Trans-(2- } \\
\text { Ethylcyclopentyl)- } \\
\text { Methanol }\end{array}$ & 20.0 & 29.9 & 24.6 & 33.0 & 37.2 & 34.6 & 32.5 & 45.3 & 36.7 & 38.1 & 43.4 & 40.0 & 31.7 & 41.7 & 35.0 & 10.1 & 14.3 & 12.4 \\
\hline Bornyl Acetate & 9.0 & 11.3 & 9.8 & 4.7 & 5.5 & 5.1 & 8.7 & 11.6 & 9.8 & 7.7 & 9.7 & 8.7 & 9.2 & 11.6 & 10.2 & 0.8 & 1.1 & 1.0 \\
\hline 4-Cyclopentene-1,3-dione & 267.1 & 328.5 & 289.9 & 260.4 & 276.8 & 269.2 & 94.5 & 145.0 & 119.6 & 77.1 & 101.9 & 89.4 & 12.9 & 20.9 & 16.6 & 39.3 & 51.5 & 44.0 \\
\hline Menthol & 2.6 & 3.7 & 3.2 & 9.1 & 11.4 & 10.4 & 0.3 & 0.5 & 0.4 & 2.7 & 3.4 & 3.1 & 2.6 & 2.6 & 2.6 & 40.0 & 45.6 & 43.8 \\
\hline 2-(2-Furanyl)-Furan & 2.3 & 3.7 & 3.0 & 3.0 & 3.6 & 3.3 & 3.6 & 4.4 & 3.9 & 1.5 & 2.5 & 1.9 & 0.8 & 1.1 & 1.0 & 0.4 & 0.6 & 0.5 \\
\hline trans-2-Octen-1-ol & 34.2 & 50.7 & 42.0 & 43.6 & 54.5 & 50.6 & 97.3 & 105.0 & 100.3 & 63.1 & 72.6 & 67.9 & 50.6 & 56.1 & 52.0 & 13.2 & 17.0 & 15.1 \\
\hline trans-2-Nonen-1-ol & 21.0 & 26.1 & 23.2 & 14.0 & 15.6 & 14.7 & 28.9 & 35.2 & 32.5 & 25.1 & 30.1 & 27.8 & 20.9 & 27.4 & 24.1 & 58.0 & 72.6 & 66.1 \\
\hline $\begin{array}{c}\text { Diethylene Glycol Ethyl } \\
\text { Ether }\end{array}$ & 61.2 & 78.3 & 66.8 & 43.4 & 53.6 & 49.0 & 63.8 & 74.1 & 68.3 & 55.4 & 62.9 & 58.8 & 42.5 & 54.8 & 46.7 & 46.1 & 61.7 & 57.2 \\
\hline $\begin{array}{l}\text { 3,5,5-Trimethyl-2- } \\
\text { Cyclopenten-1-one }\end{array}$ & 11.2 & 19.2 & 15.1 & 10.7 & 14.8 & 12.4 & 11.1 & 21.2 & 16.4 & 16.4 & 19.3 & 17.5 & 11.5 & 18.1 & 14.4 & 11.3 & 16.6 & 14.3 \\
\hline$\beta$-Cyclocitral & 2.9 & 3.4 & 3.2 & 4.4 & 4.8 & 4.5 & 9.4 & 10.9 & 10.2 & 8.5 & 10.3 & 9.2 & 5.6 & 6.9 & 6.3 & 14.9 & 21.7 & 17.5 \\
\hline Benzeneacetaldehyde & 86.2 & 100.6 & 92.9 & 34.8 & 39.3 & 36.6 & 16.9 & 19.5 & 18.5 & 13.9 & 18.0 & 16.2 & 11.5 & 14.0 & 13.1 & 75.7 & 82.2 & 79.1 \\
\hline 1-Nonanol & 23.9 & 35.2 & 28.1 & 36.5 & 43.7 & 39.9 & 29.1 & 34.1 & 31.6 & 26.7 & 32.7 & 29.7 & 73.9 & 84.8 & 80.3 & 8.8 & 9.9 & 9.4 \\
\hline Furfuryl Alcohol & 56.0 & 61.6 & 59.2 & 69.3 & 89.3 & 83.6 & 71.8 & 84.4 & 76.9 & 39.9 & 54.0 & 47.0 & 10.3 & 13.9 & 12.6 & 49.3 & 55.5 & 53.5 \\
\hline Phoracanthol & 65.8 & 81.8 & 71.4 & 12.0 & 17.5 & 14.7 & 8.9 & 11.4 & 10.3 & 11.0 & 14.1 & 13.0 & 9.4 & 11.5 & 10.1 & 5.2 & 6.5 & 5.9 \\
\hline cis-3-Nonen-1-ol & 17.6 & 23.2 & 19.6 & 7.7 & 10.0 & 8.9 & 10.1 & 12.5 & 11.0 & 15.6 & 22.7 & 18.9 & 12.5 & 16.7 & 14.4 & 11.4 & 16.5 & 14.0 \\
\hline cis-Piperitol & 2.8 & 3.7 & 3.3 & 1.6 & 2.5 & 2.0 & 0.5 & 0.6 & 0.6 & 26.2 & 31.4 & 28.3 & 2.3 & 2.8 & 2.5 & 37.5 & 48.0 & 42.8 \\
\hline p-Methoxy-Styrene & 5.3 & 7.4 & 6.0 & 1.5 & 1.7 & 1.6 & 23.0 & 30.2 & 25.6 & 4.0 & 5.6 & 4.7 & 0.3 & 0.4 & 0.4 & 2.7 & 3.6 & 3.2 \\
\hline $\begin{array}{l}\text { (2Z)-3-Ethyl-2,4- } \\
\text { Pentadien-1-ol }\end{array}$ & 9.0 & 11.5 & 9.9 & 6.6 & 7.0 & 6.9 & 6.5 & 6.9 & 6.6 & 6.1 & 8.3 & 7.4 & 4.2 & 4.9 & 4.4 & 1.3 & 2.0 & 1.5 \\
\hline $\begin{array}{l}\text { Butylene Glycol } \\
\text { Monobutyl Ether }\end{array}$ & 13.0 & 18.8 & 15.6 & 11.3 & 17.7 & 14.4 & 8.7 & 10.1 & 9.1 & 10.7 & 16.9 & 13.0 & 8.2 & 9.1 & 8.6 & 1.1 & 1.4 & 1.3 \\
\hline$\alpha$-Terpineol & 1.9 & 2.5 & 2.2 & 2.2 & 2.3 & 2.2 & 0.9 & 1.2 & 1.0 & 0.5 & 0.6 & 0.5 & 0.1 & 0.2 & 0.2 & 4.4 & 5.8 & 5.1 \\
\hline 1,5-Cyclooctanedione & 2.0 & 2.2 & 2.1 & 0.5 & 4.3 & 2.4 & 1.9 & 2.2 & 2.1 & 2.1 & 2.9 & 2.6 & 2.2 & 2.9 & 2.7 & 4.1 & 5.1 & 4.5 \\
\hline$\gamma$-Terpinene & 1.7 & 2.1 & 1.9 & 3.2 & 4.5 & 3.6 & 5.8 & 7.5 & 6.5 & 3.0 & 3.8 & 3.4 & 4.4 & 5.7 & 5.0 & 1.6 & 2.0 & 1.7 \\
\hline
\end{tabular}




\begin{tabular}{|c|c|c|c|c|c|c|c|c|c|c|c|c|c|c|c|c|c|c|}
\hline 2-Propenoic Acid & 8.7 & 14.3 & 10.8 & 1.0 & 1.1 & 1.0 & 0.5 & 0.6 & 0.5 & 0.2 & 0.2 & 0.2 & 0.5 & 0.5 & 0.5 & 0.9 & 1.3 & 1.1 \\
\hline 2-Undecanol & 4.1 & 4.9 & 4.5 & 76.1 & 87.0 & 79.7 & 2.1 & 3.7 & 2.9 & 0.9 & 1.1 & 1.0 & 21.7 & 27.5 & 24.6 & 1.6 & 2.7 & 2.2 \\
\hline 3-Ethyl-Benzaldehyde & 5.1 & 6.6 & 5.7 & 4.2 & 5.0 & 4.6 & 5.6 & 6.5 & 6.1 & 4.9 & 5.7 & 5.3 & 3.2 & 4.4 & 3.5 & 5.9 & 7.3 & 6.5 \\
\hline 3-Hydroxy-Benzaldehyde & 13.0 & 22.0 & 16.2 & 1.7 & 2.1 & 1.8 & 4.4 & 5.2 & 4.7 & 11.7 & 14.8 & 12.7 & 0.9 & 1.0 & 1.0 & 1.5 & 1.8 & 1.6 \\
\hline trans-2-Nonene & 3.1 & 3.6 & 3.4 & 4.2 & 5.4 & 4.8 & 0.4 & 0.5 & 0.4 & 1.4 & 1.6 & 1.5 & 1.0 & 1.7 & 1.4 & 1.7 & 2.0 & 1.8 \\
\hline 2-Methyl-2-Pentenal & 14.1 & 18.0 & 15.8 & 6.7 & 7.8 & 7.3 & 6.5 & 7.1 & 6.7 & 8.7 & 13.4 & 10.3 & 3.6 & 4.1 & 3.8 & 8.3 & 11.1 & 9.7 \\
\hline cis-5-Decenol & 5.0 & 5.6 & 5.2 & 96.0 & 121.9 & 108.0 & 1.5 & 2.9 & 2.2 & 4.0 & 4.6 & 4.4 & 83.3 & 94.5 & 88.4 & 13.8 & 20.0 & 16.2 \\
\hline trans-Piperitol & 2.0 & 2.6 & 2.3 & 4.4 & 5.6 & 5.1 & 0.8 & 1.0 & 0.9 & 19.6 & 26.7 & 22.6 & 1.9 & 2.4 & 2.1 & 30.1 & 36.1 & 33.7 \\
\hline 4-Ethyl-Benzaldehyde & 3.4 & 4.0 & 3.6 & 2.7 & 3.4 & 3.0 & 4.9 & 5.8 & 5.4 & 3.4 & 3.5 & 3.4 & 3.1 & 3.4 & 3.3 & 3.9 & 5.0 & 4.7 \\
\hline Naphthalene & 14.9 & 19.1 & 16.7 & 7.5 & 8.3 & 7.9 & 18.4 & 22.1 & 19.8 & 12.8 & 18.1 & 14.6 & 8.0 & 11.1 & 9.9 & 1.1 & 1.6 & 1.4 \\
\hline 1-Decanol & 30.6 & 39.9 & 33.6 & 16.6 & 20.4 & 18.1 & 15.5 & 20.8 & 18.3 & 13.9 & 18.2 & 15.5 & 17.3 & 22.8 & 20.8 & 18.1 & 20.5 & 19.3 \\
\hline Cyclodecane & 98.1 & 150.3 & 121.5 & 84.7 & 94.3 & 88.4 & 62.6 & 68.7 & 66.1 & 65.1 & 74.1 & 69.7 & 32.2 & 35.8 & 34.0 & 39.3 & 44.3 & 42.0 \\
\hline trans,trans-2,4-Decadienal & 11.7 & 19.7 & 15.3 & 19.4 & 25.3 & 21.4 & 49.2 & 57.2 & 52.2 & 14.8 & 20.9 & 17.3 & 16.5 & 23.4 & 20.7 & 3.8 & 4.4 & 4.2 \\
\hline 1,2-Cyclopentanedione & 531.1 & 662.6 & 574.6 & 417.1 & 457.3 & 439.9 & 182.0 & 207.8 & 193.8 & 554.1 & 644.1 & 596.9 & 209.6 & 247.4 & 225.7 & 212.8 & 269.4 & 240.3 \\
\hline Cyclodecene & 2.0 & 2.6 & 2.3 & 2.0 & 2.2 & 2.1 & 1.4 & 2.0 & 1.7 & 3.1 & 3.6 & 3.3 & 1.5 & 2.0 & 1.9 & 0.5 & 0.6 & 0.5 \\
\hline cis-4-Decenol & 1.5 & 1.9 & 1.8 & 2.9 & 3.4 & 3.1 & 0.5 & 0.7 & 0.6 & 0.7 & 0.8 & 0.8 & 1.2 & 1.6 & 1.4 & 2.6 & 3.2 & 2.8 \\
\hline 1-Phenyl-2-Butanone & 7.3 & 9.0 & 7.9 & 6.8 & 7.7 & 7.3 & 14.3 & 16.0 & 15.2 & 5.9 & 8.3 & 6.7 & 12.5 & 13.8 & 13.0 & 7.9 & 9.3 & 8.6 \\
\hline Phenethyl Alcohol & 11.8 & 16.4 & 13.8 & 2.1 & 2.5 & 2.3 & 5.1 & 5.8 & 5.5 & 19.6 & 24.8 & 21.3 & 4.2 & 4.3 & 4.3 & 7.6 & 9.8 & 8.8 \\
\hline trans,cis-2,4-Decadienal & 7.9 & 10.1 & 8.9 & 12.5 & 15.9 & 14.0 & 40.3 & 47.8 & 43.4 & 11.6 & 15.3 & 12.9 & 10.8 & 17.5 & 14.1 & 2.9 & 3.3 & 3.2 \\
\hline 2-Cyclohexenol & 73.6 & 86.0 & 79.2 & 36.9 & 48.0 & 42.2 & 4.0 & 5.0 & 4.5 & 64.0 & 77.9 & 71.7 & 10.6 & 12.5 & 11.6 & 8.1 & 9.0 & 8.4 \\
\hline 2-Ethyl Hexanoic Acid & 20.2 & 29.3 & 24.3 & 4.1 & 4.6 & 4.3 & 48.6 & 56.2 & 52.5 & 15.0 & 22.2 & 18.6 & 1.0 & 1.2 & 1.1 & 13.5 & 17.7 & 15.7 \\
\hline $\begin{array}{l}\text { 3-Methyl-1,2- } \\
\text { Cyclopentanedione }\end{array}$ & 26.3 & 35.9 & 3 & 45.8 & 53.9 & 49.7 & 8 & 9.9 & 8 & 6 & 73.7 & 67.3 & 2.0 & 2.8 & 2.5 & 6.7 & 5 & 7.2 \\
\hline trans-Cinnamaldehyde & 9.1 & 12.1 & 10.4 & 5.5 & 5.9 & 5.8 & 7.6 & 8.9 & 8.2 & 8.7 & 9.6 & 9.2 & 4.7 & 5.1 & 4.8 & 7.4 & 8.7 & 8.0 \\
\hline Heptanoic Acid & 72.8 & 86.5 & 80.1 & 29.8 & 37.5 & 33.7 & 72.1 & 89.4 & 83.7 & 48.1 & 61.8 & 52.7 & 1.3 & 1.9 & 1.5 & 95.5 & 116.4 & 104.7 \\
\hline Mequinol & 26.7 & 30.6 & 28.0 & 19.7 & 25.3 & 22.2 & 78.4 & 84.5 & 82.8 & 81.7 & 132.1 & 109.9 & 44.9 & 51.4 & 46.8 & 7.3 & 8.5 & 7.7 \\
\hline $\begin{array}{c}2,4,5- \\
\text { Trihydroxypyrimidine }\end{array}$ & 63.6 & 72.2 & 69.0 & 9.8 & 17.0 & 13.5 & 10.0 & 11.7 & 10.6 & 7.5 & 9.1 & 8.5 & 11.1 & 17.5 & 14.1 & 14.4 & 20.2 & 16.8 \\
\hline cis-Cinnamaldehyde & 8.2 & 9.8 & 9.1 & 8.0 & 9.2 & 8.7 & 7.3 & 8.5 & 7.8 & 8.1 & 9.0 & 8.5 & 4.0 & 5.0 & 4.5 & 7.9 & 9.2 & 8.6 \\
\hline Benzenemethanol & 45.2 & 55.3 & 48.4 & 20.4 & 28.3 & 24.7 & 102.7 & 150.7 & 123.7 & 77.4 & 92.4 & 83.6 & 38.3 & 48.7 & 41.8 & 10.8 & 20.5 & 14.8 \\
\hline Mesitaldehyde & 5.0 & 6.4 & 5.5 & 2.1 & 3.0 & 2.5 & 5.8 & 7.1 & 6.3 & 5.9 & 6.1 & 6.0 & 2.9 & 3.9 & 3.4 & 5.7 & 6.8 & 6.2 \\
\hline
\end{tabular}




\begin{tabular}{|c|c|c|c|c|c|c|c|c|c|c|c|c|c|c|c|c|c|c|}
\hline 2-Ethylhexyl Octanoate & 1.0 & 1.6 & 1.3 & 2.8 & 3.2 & 3.0 & 0.7 & 0.9 & 0.8 & 8.9 & 10.0 & 9.5 & 4.2 & 5.0 & 4.5 & 3.9 & 4.4 & 4.1 \\
\hline Benzeneethanol & 89.1 & 126.9 & 106.3 & 28.9 & 35.0 & 30.9 & 42.2 & 53.5 & 47.9 & 58.8 & 73.0 & 63.5 & 28.2 & 34.9 & 31.1 & 37.8 & 47.5 & 43.6 \\
\hline 1,4-Butanediol & 82.3 & 97.6 & 88.6 & 64.9 & 77.5 & 68.8 & 59.3 & 65.9 & 61.5 & 62.4 & 74.8 & 68.1 & 35.7 & 43.2 & 38.9 & 47.2 & 59.5 & 51.6 \\
\hline$\beta$-Ionone & 12.5 & 16.7 & 14.3 & 11.3 & 12.2 & 11.7 & 35.5 & 46.6 & 40.5 & 19.5 & 31.2 & 25.4 & 16.4 & 21.7 & 18.8 & 48.3 & 63.8 & 52.7 \\
\hline 2-Methyl-1,4-Benzenediol & 20.2 & 28.5 & 23.9 & 12.8 & 19.2 & 15.9 & 3.2 & 4.1 & 3.7 & 19.6 & 28.1 & 23.1 & 0.7 & 7.3 & 3.9 & 1.4 & 2.0 & 1.8 \\
\hline 2-Ethyl Heptanoic Acid & 0.9 & 1.2 & 1.0 & 5.2 & 5.7 & 5.3 & 1.5 & 1.7 & 1.6 & 2.4 & 3.1 & 2.7 & 9.9 & 17.2 & 12.9 & 1.5 & 2.2 & 1.8 \\
\hline 1-Dodecanol & 9.4 & 13.9 & 11.6 & 5.0 & 6.9 & 5.9 & 10.5 & 15.5 & 13.0 & 3.0 & 3.9 & 3.5 & 5.4 & 6.2 & 5.8 & 11.4 & 13.5 & 12.6 \\
\hline Benzalacetone & 18.1 & 19.1 & 18.5 & 8.6 & 9.6 & 9.0 & 9.4 & 12.2 & 10.5 & 8.7 & 10.3 & 9.4 & 4.9 & 5.7 & 5.2 & 10.2 & 12.1 & 11.3 \\
\hline Maltol & 92.4 & 157.8 & 124.5 & 127.1 & 179.1 & 150.8 & 62.7 & 69.9 & 65.9 & 181.4 & 246.6 & 212.9 & 2.7 & 23.4 & 12.7 & 66.6 & 78.8 & 74.7 \\
\hline 2-Acetylpyrrole & 17.8 & 19.5 & 18.6 & 19.7 & 21.7 & 20.4 & 0.6 & 0.7 & 0.6 & 11.4 & 14.1 & 12.8 & 0.9 & 1.2 & 1.0 & 2.0 & 2.7 & 2.4 \\
\hline 2,4-Decadien-1-ol & 8.1 & 12.1 & 9.6 & 7.8 & 8.2 & 8.0 & 7.4 & 8.7 & 8.0 & 8.9 & 10.4 & 9.3 & 8.4 & 10.0 & 9.3 & 4.0 & 4.7 & 4.3 \\
\hline Levoglucosenone & 8.8 & 11.6 & 9.7 & 12.5 & 17.1 & 14.6 & 7.9 & 9.3 & 8.7 & 13.5 & 17.1 & 16.0 & 0.9 & 1.5 & 1.2 & 3.8 & 4.4 & 4.2 \\
\hline Phenol & 32.4 & 51.1 & 41.4 & 36.3 & 47.1 & 42.9 & 48.8 & 67.1 & 57.5 & 20.3 & 29.0 & 24.6 & 35.9 & 44.6 & 40.0 & 35.3 & 40.4 & 38.6 \\
\hline $\begin{array}{l}\text { 1-(2-Furanyl)-2-Hydroxy- } \\
\text { Ethanone }\end{array}$ & 40.8 & 50.8 & 47.0 & 22.5 & 28.8 & 25.7 & 4.1 & 5.3 & 4.8 & 9.2 & 10.2 & 9.8 & 24.0 & 27.2 & 25.3 & 5.9 & 7.1 & 6.5 \\
\hline 4,6-Dimethyl-Dodecane & 18.9 & 29.1 & 22.9 & 30.3 & 32.5 & 31.1 & 3.3 & 4.0 & 3.6 & 24.2 & 31.3 & 28.0 & 1.3 & 2.0 & 1.6 & 9.9 & 12.6 & 11.3 \\
\hline Ethyl Decanoate & 5.3 & 6.8 & 6.0 & 5.1 & 6.0 & 5.5 & 4.9 & 5.5 & 5.2 & 5.8 & 7.4 & 6.5 & 8.9 & 10.0 & 9.6 & 1.4 & 2.0 & 1.6 \\
\hline 1-Methyl-4-Piperidinone & 1.2 & 1.8 & 1.5 & 0.5 & 0.6 & 0.6 & 0.3 & 0.4 & 0.4 & 1.3 & 1.9 & 1.7 & 0.6 & 0.7 & 0.7 & 4.1 & 4.8 & 4.5 \\
\hline $\begin{array}{l}\text { 2,2-Dimethyl-3- } \\
\text { Heptanone }\end{array}$ & 2.5 & 3.2 & 2.8 & 100.3 & 112.5 & 107.6 & 3.7 & 4.2 & 4.0 & 98.3 & 198.8 & 143.2 & 1.0 & 1.5 & 1.2 & 36.2 & 40.9 & 38.0 \\
\hline Octanoic Acid & 29.8 & 43.1 & 34.8 & 1.3 & 1.6 & 1.4 & 15.4 & 19.7 & 18.1 & 10.7 & 18.1 & 15.0 & 1.4 & 1.8 & 1.6 & 15.6 & 21.7 & 18.0 \\
\hline $\begin{array}{l}\text { 1,3-Dihydroxy-2- } \\
\text { Propanone }\end{array}$ & 78.0 & 102.4 & 87.6 & 36.4 & 43.4 & 41.1 & 1.1 & 2.1 & 1.7 & 126.7 & 185.1 & 158.3 & 1.1 & 1.3 & 1.2 & 1.2 & 1.6 & 1.4 \\
\hline 2-Phenoxy-Ethanol & 6.8 & 8.2 & 7.3 & 5.3 & 6.5 & 5.9 & 13.6 & 18.1 & 16.5 & 16.2 & 17.9 & 17.3 & 11.6 & 14.7 & 13.5 & 49.1 & 56.2 & 52.7 \\
\hline Isophthalaldehyde & 4.1 & 5.3 & 4.7 & 4.3 & 5.0 & 4.6 & 4.5 & 5.5 & 5.1 & 3.5 & 4.3 & 4.0 & 3.0 & 3.7 & 3.4 & 5.9 & 6.5 & 6.3 \\
\hline Cyclotetradecane & 8.5 & 10.3 & 9.0 & 2.1 & 2.9 & 2.5 & 4.6 & 5.1 & 4.9 & 7.1 & 8.0 & 7.5 & 1.9 & 3.1 & 2.6 & 2.8 & 3.3 & 3.1 \\
\hline Nonanoic Acid & 14.2 & 21.7 & 17.6 & 1.4 & 1.8 & 1.6 & 40.0 & 49.2 & 44.8 & 13.2 & 16.6 & 14.5 & 1.2 & 1.3 & 1.2 & 50.4 & 58.3 & 54.1 \\
\hline 2-Ethyl-Phenol & 14.4 & 21.3 & 17.7 & 11.9 & 15.9 & 13.7 & 24.8 & 28.4 & 26.5 & 28.1 & 36.4 & 34.2 & 22.7 & 30.2 & 24.8 & 10.9 & 14.1 & 12.5 \\
\hline $\begin{array}{l}\text { 2-Methoxy-4-Vinyl- } \\
\text { Phenol }\end{array}$ & 60.8 & 67.4 & 63.3 & 50.9 & 59.8 & 54.3 & 26.6 & 32.4 & 30.7 & 72.7 & 85.5 & 80.4 & 64.2 & 72.3 & 67.0 & 4.1 & 4.6 & 4.4 \\
\hline 4-Methylimidazole & 1.0 & 1.3 & 1.1 & 17.8 & 24.2 & 20.8 & 24.0 & 32.6 & 26.6 & 13.2 & 21.4 & 17.9 & 8.6 & 11.7 & 9.8 & 89.3 & 110.5 & 101.7 \\
\hline m-tert-Butyl-Toluene & 15.0 & 18.9 & 16.7 & 16.2 & 18.4 & 17.1 & 16.6 & 19.6 & 18.3 & 11.7 & 15.0 & 13.3 & 9.5 & 11.5 & 10.4 & 15.9 & 16.9 & 16.3 \\
\hline
\end{tabular}




\begin{tabular}{|c|c|c|c|c|c|c|c|c|c|c|c|c|c|c|c|c|c|c|}
\hline Syringol & 18.8 & 24.7 & 21.4 & 17.7 & 19.6 & 18.6 & 12.3 & 14.0 & 13.4 & 19.8 & 24.5 & 22.3 & 10.8 & 13.8 & 12.1 & 13.6 & 14.7 & 14.0 \\
\hline Decanoic Acid & 27.9 & 35.2 & 32.7 & 5.2 & 6.6 & 5.7 & 27.0 & 34.4 & 31.3 & 27.4 & 32.5 & 30.2 & 1.4 & 1.9 & 1.7 & 30.2 & 39.7 & 34.1 \\
\hline $\begin{array}{c}\text { 3-Hydroxy-2,3-Dihydro- } \\
\text { Maltol }\end{array}$ & 57.6 & 66.2 & 61.2 & 17.8 & 19.2 & 18.7 & 33.2 & 43.9 & 40.0 & 19.0 & 26.0 & 21.8 & 1.0 & 1.4 & 1.3 & 4.4 & 5.8 & 4.9 \\
\hline 5-Hydroxy-Maltol & 34.3 & 39.4 & 36.8 & 67.0 & 72.7 & 70.1 & 27.5 & 43.9 & 36.0 & 30.1 & 39.6 & 34.3 & 1.3 & 1.8 & 1.5 & 43.8 & 49.6 & 45.9 \\
\hline Triethylene Glycol & 0.9 & 1.2 & 1.1 & 0.9 & 1.4 & 1.2 & 5.3 & 6.1 & 5.7 & 11.6 & 14.8 & 13.5 & 1.5 & 2.1 & 1.9 & 4.2 & 5.2 & 4.8 \\
\hline Benzoic Acid & 12.5 & 20.4 & 16.7 & 5.9 & 6.8 & 6.4 & 5.2 & 5.7 & 5.4 & 12.1 & 15.1 & 13.9 & 19.1 & 21.9 & 20.4 & 2.9 & 3.4 & 3.2 \\
\hline 2-Methyl-Benzaldehyde & 190.8 & 224.3 & 201.6 & 124.6 & 192.5 & 158.7 & 216.0 & 268.2 & 242.7 & 470.7 & 610.9 & 533.6 & 515.4 & 623.5 & 573.5 & 15.4 & 21.7 & 19.1 \\
\hline Undecanoic Acid & 28.3 & 35.1 & 32.1 & 4.7 & 5.8 & 5.1 & 28.1 & 31.6 & 30.4 & 22.0 & 29.9 & 27.0 & 1.5 & 2.0 & 1.7 & 63.3 & 75.3 & 70.1 \\
\hline 4-Pyridinol & 91.6 & 133.0 & 104.5 & 4.4 & 5.5 & 5.1 & 37.0 & 46.4 & 43.0 & 83.6 & 98.8 & 91.0 & 16.9 & 19.1 & 17.8 & 61.2 & 76.0 & 69.3 \\
\hline 4-Amino-Phenol & 48.4 & 55.5 & 50.9 & 5.7 & 6.4 & 6.0 & 1.5 & 2.1 & 1.8 & 35.7 & 42.0 & 39.0 & 13.1 & 18.4 & 16.0 & 3.7 & 5.0 & 4.3 \\
\hline
\end{tabular}

Table S5. VOCs from regional cultivars samples previously identified in others sugarcane-based products.

\begin{tabular}{|c|c|c|c|c|c|c|c|c|c|c|}
\hline \multirow[b]{2}{*}{ Volatile Organic Compound } & \multicolumn{10}{|c|}{ Sugarcane Based-Products } \\
\hline & $\begin{array}{c}\text { SCH } \\
1\end{array}$ & $\begin{array}{c}\text { Juice } \\
2-7\end{array}$ & $\begin{array}{l}\text { Sugar } \\
6,8-11\end{array}$ & $\begin{array}{c}\text { Treacle } \\
12\end{array}$ & $\begin{array}{c}\text { Molasses } \\
13-15\end{array}$ & $\underset{1,6,16-18}{\text { Syrup }}$ & $\begin{array}{l}\text { Rum } \\
19-25\end{array}$ & $\begin{array}{c}\text { Infusion } \\
26\end{array}$ & $\begin{array}{c}\text { Alcoholic } \\
\text { Fermented } \\
\text { Beverage } \\
27,28\end{array}$ & $\begin{array}{c}\text { All } \\
\text { Products }\end{array}$ \\
\hline Pentane & & & & & & & & & & 0 \\
\hline 1-Pentene & & & & & & & & & & 0 \\
\hline 2-Methyl-Pentane & & & & & & & & & & 0 \\
\hline Hexane & & & & & & & & & & 0 \\
\hline Ethyl Ether & & & & & & & & & & 0 \\
\hline cis-4-Methyl-2-Pentene & & & & & & & & & & 0 \\
\hline cis-Piperylene & & & & & & & & & & 0 \\
\hline 2-Methyl-1-Pentene & & & & & & & & & & 0 \\
\hline cis-3-Methyl-2-Pentene & & & & & & & & & & 0 \\
\hline 3-Methyl-Hexane & & & & & & & & & & 0 \\
\hline 1,4-Pentadiene & & & & & & & & & & 0 \\
\hline Methanethiol & & & & & & & & & & 0 \\
\hline
\end{tabular}


tert-Butyl Ethyl Ether

$$
\text { Ethanal }
$$

Dimethyl Sulfide

X

$\mathrm{X}$

4-Methyl-Heptane

Octane

Propanal

Furan

2,4-Dimethyl-Heptane

2-Methyl-Propanal

2-Propanone

Methyl Acetate

2-Methyl-2-Heptene

$$
\text { Crotonal }
$$

2,3-Dimethyl-Heptane

4-Methyl-Octane

2-Methyl-Furan

2,4-Dimethyl-1-Heptene

Ethyl Acetate

Nonane

2-Butanone

2-Methyl-Butanal

3-Methyl-Butanal

Ethyl Alcohol

Benzene

3-Ethyl-Heptane

2-Ethyl-Furan

Ethyl Propanoate
X

X

X

X

$\mathrm{X}$

X

$\mathrm{X}$

$\mathrm{X}$

X

$\mathrm{X}$

X

X

$$
\begin{aligned}
& X \\
& X
\end{aligned}
$$$$
\mathrm{X}
$$$$
\text { X }
$$$$
\begin{aligned}
& \mathrm{X} \\
& \mathrm{X}
\end{aligned}
$$$$
\mathrm{X}
$$

Ethyl 2-Methylpropanoate 
3-Pentanone

Pentanal

2,3-Butanedione

2,3,4-Trimethyl-Hexane

$$
\text { Decane }
$$

2,6-Dimethyl-Nonane

2,5-Dimethyl-Nonane

4-Methyl-2-Pentanone

2-Methy-2-Butanol

2-Methylpropyl Acetate $\alpha$-Pinene

4,4-Dimethyl-2-Pentanone

1-Penten-3-one

5-Butyl-Nonane

3,6-Dimethyl-Decane

2-Methyl-3-Buten-2-ol

5-Methyl-Decane

4-Methyl-Decane

2,3-Pentanedione

Methyl 3,3-Dimethylbutanoate

$$
\text { 2-Hexanone }
$$

4-Methyl-Undecane

$$
\text { Hexanal }
$$

2-Methyl-1-Propanol

2-Methyl-2-Pentanol

Ethyl-Benzene

3-Methylbutyl Acetate o-Xylene

$\mathrm{X}$ 
$\mathrm{p}$-Xylene

5-Methyl-2-Hexanone

Methyl 2-Ethylpentanoate

1-Butanol

$\alpha$-Phellandrene

2-Methyl-3-Pentanol

1-Penten-3-ol

$\alpha$-Terpinene

Butyl Prop-2-enoate

$\mathrm{m}$-Xylene

2-Heptanone

Heptanal

D-Limonene

3-Hexanol

$\beta$-Phellandrene

Eucalyptol

m-Menthane

4-Methyl-2-Heptanone

3-Methyl-2-Pentanol

2-Methyl-1-Butanol

3-Methyl-1-Butanol

o-Ethyltoluene

m-Ethyltoluene

$$
\text { 2-Hexanol }
$$

Ethylene Glycol Ethyl Ether 6-Methyl-2-Heptanone

Pseudocumene

4,6-Dimethyl-2-Heptanone

2,3-Dimethyl-2-Hexanol

$\mathrm{X}$

$\mathrm{X}$
X
X

0 
3-Octanone

Styrene

5-Methyl-2-Heptanone

1-Pentanol

X

p-Ethyltoluene

p-Cymene

$$
\text { Mesitylene }
$$

4-Methyl-4-Heptanol

4-Heptanol

2-Octanone

$$
\text { Octanal }
$$

3-Hydroxy-2-Butanone

1-Octen-3-one

trans-2-Pentenol

2,2,6-Trimethyl-Cyclohexanone

2-Heptanol

cis-2-Pentenol

1-Hydroxy-2-Propanone

5-Methyl-4-Hexen-3-one

Hemimellitene

6-Methyl-5-Hepten-2-one

2-Pentene

1-Hexanol

2-Cyclopenten-1-one

2-Methyl-2-Cyclopenten-1-one $\beta$-Methy-Styrene

$$
\text { 4-Octanol }
$$

cis-3-Hexen-1-ol

2-Nonanone

Nonanal 
Ethylene Glycol Butyl Ether

1-Ethyl-1-Methyl-Cyclopentane

3-Octen-2-one

2-Octanol

3-Ethyl-2-Methyl-1,3-Hexadiene

1,3-Bis(1,1-Dimethylethyl)-Benzene

trans-2-Octenal

m-Ethyl-Styrene

5-Methyl-2-Heptanone

2-Decanone

1-Octen-3-ol

trans,trans-2,4-Heptadienal

1-Heptanol

cis,cis-3,5-Octadiene

Methyl 2-Oxopropanoate

Dihydromyrcenol

trans-Menthone

Furfural

Ethanoic Acid

2-Ethyl-1-Hexanol

Decanal

Furfuryl Formate

trans-2-Hepten-1-ol

2-Nonanol

1-(2-Furanyl)-Ethanone

3,5-Octadien-2-one

Benzaldehyde

Furfuryl Acetate

Linalool

$\begin{array}{ll}X & X \\ X & X \\ X & X\end{array}$

X

$\mathrm{X}$

X

X

X

X

X 
1-Octanol

trans-p-2-Menthen-1-ol

Propanoic Acid

2,3-Butanediol

Dimethyl Sulfoxide

m-Menth-3-ene

Trans-(2-Ethylcyclopentyl)-Methanol

Bornyl Acetate

4-Cyclopentene-1,3-dione

Menthol

2-Undecanone

2-(2-Furanyl)-Furan

trans-2-Octen-1-ol

trans-2-Nonen-1-ol

Diethylene Glycol Ethyl Ether

3,5,5-Trimethyl-2-Cyclopenten-1-one cis-p-2-Menthen-1-ol

$\beta$-Cyclocitral

Benzeneacetaldehyde

1-Nonanol

Furfuryl Alcohol

Phoracanthol

cis-3-Nonen-1-ol

cis-Piperitol

p-Methoxy-Styrene

(2Z)-3-Ethyl-2,4-Pentadien-1-ol

Butylene Glycol Monobutyl Ether $\alpha$-Terpineol

1,5-Cyclooctanedione $\gamma$-Terpinene 
trans-2-Decenol

2-Propenoic Acid

2-Undecanol

3-Ethyl-Benzaldehyde

3-Hydroxy-Benzaldehyde trans-2-Nonene

2-Methyl-2-Pentenal cis-5-Decenol

trans-Piperitol

4-Ethyl-Benzaldehyde

Naphthalene

1-Decanol

Cyclodecane

trans,trans-2,4-Decadienal

1,2-Cyclopentanedione

Cyclodecene

cis-4-Decenol

1-Phenyl-2-Butanone

Phenethyl Alcohol

trans,cis-2,4-Decadienal

2-Cyclohexenol

2-Ethyl Hexanoic Acid

3-Methyl-1,2-Cyclopentanedione

trans-Cinnamaldehyde

Heptanoic Acid

Mequinol

X

X

$\mathrm{X}$

X

cis-Cinnamaldehyde

Benzenemethanol

Mesitaldehyde 
2-Ethylhexyl Octanoate

Benzeneethanol

1,4-Butanediol

$\beta$-Ionone

2-Methyl-1,4-Benzenediol

2-Ethyl Heptanoic Acid

1-Dodecanol

Benzalacetone

Maltol

2-Acetylpyrrole

2,4-Decadien-1-ol

Levoglucosenone Phenol

1-(2-Furanyl)-2-Hydroxy-Ethanone

4,6-Dimethyl-Dodecane

Ethyl Decanoate

1-Methyl-4-Piperidinone

2,2-Dimethyl-3-Heptanone

Octanoic Acid

1,3-Dihydroxy-2-Propanone

2-Phenoxy-Ethanol

Isophthalaldehyde

Cyclotetradecane

Nonanoic Acid

2-Ethyl-Phenol

2-Methoxy-4-Vinyl-Phenol

4-Methylimidazole

m-tert-Butyl-Toluene

Syringol

Decanoic Acid

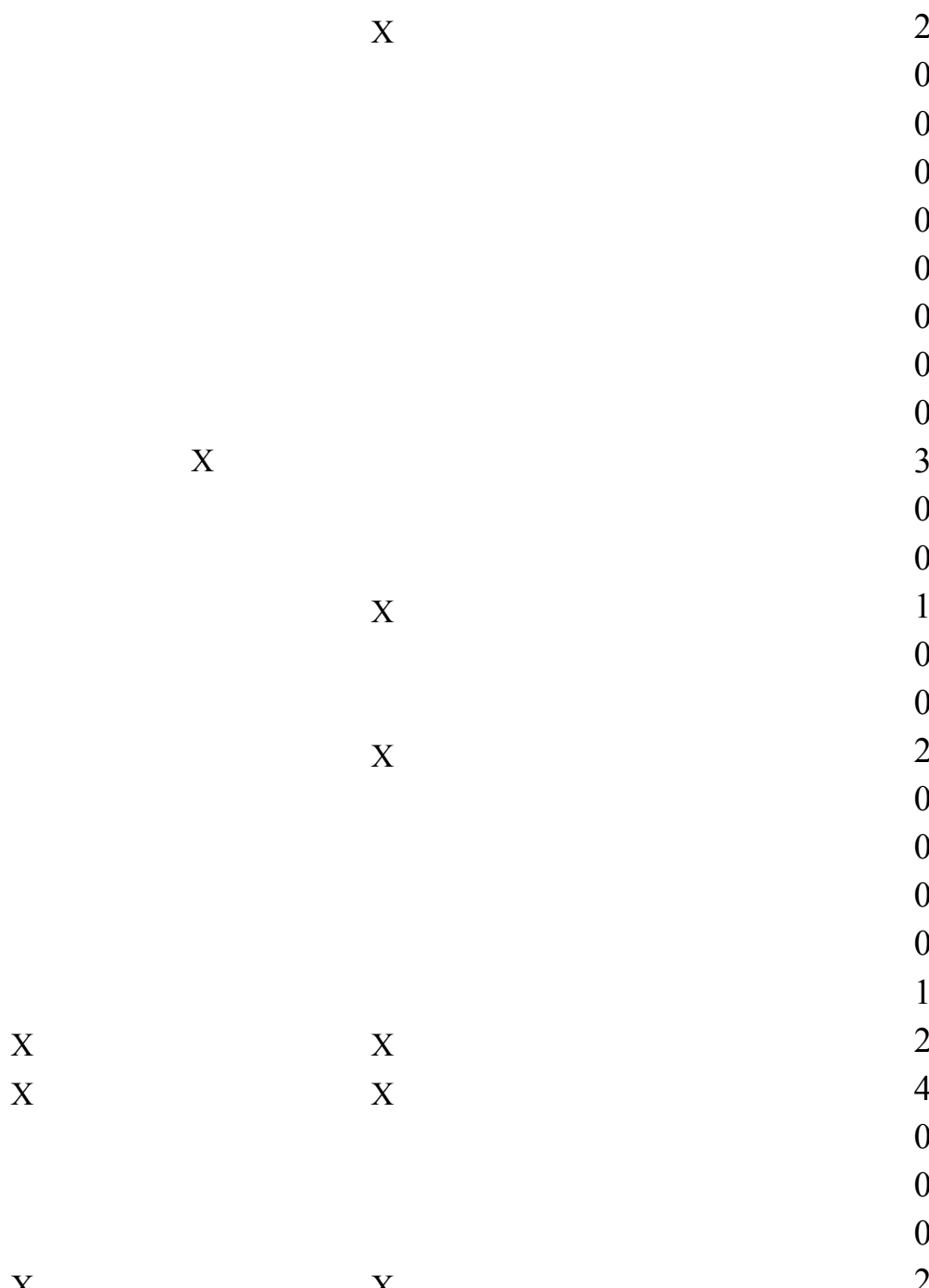


Undecanoic Acid

4-Pyridinol

4-Amino-Phenol

$\mathrm{X}$ - Identified in sugarcane based-product.

Total

37

56

34

16

42

4

\section{References}

(1) Silva, P.; Freitas, J.; Silva, C. L.; Perestrelo, R.; Nunes, F. M.; Câmara, J. S. Establishment of Authenticity and Typicality of Sugarcane Honey Based on Volatile Profile and Multivariate Analysis. Food Control 2017, 73, 1176-1188. https://doi.org/10.1016/j.foodcont.2016.10.035.

(2) Wang, L.; Wang, P.; Deng, W.; Cai, J.; Chen, J. Evaluation of Aroma Characteristics of Sugarcane (Saccharum Officinarum L.) Juice Using Gas Chromatography-Mass Spectrometry and Electronic Nose. LWT 2019, 108, 400-406. https://doi.org/10.1016/j.lwt.2019.03.089.

(3) Yang, H. F.; Wang, S. L.; Yu, S. J.; Zeng, X. A.; Sun, D. W. Characterization and Semiquantitative Analysis of Volatile Compounds in Six Varieties of Sugarcane Juice. Int. J. Food Eng. 2014, 10 (4), 821-828. https://doi.org/10.1515/ijfe-2014-0105.

(4) Sharma, M. D.; Rautela, I.; Sharma, N.; Gahlot, M.; Koshy, E. P. GC-MM Analysis of Phytocomponents in Juice Sample of Indian Cane: Saccharum Barberi. Int. J. Pharm. Sci. Res. 2015, 6 (12), 5147-5153. https://doi.org/10.13040/IJPSR.0975-8232.6(12).5147-53.

(5) Tokitomo, Y.; Kobayashi, A.; Yamanishi, T. Aroma Components of Fresh Sugar Cane Juice. Agric. Biol. Chem. 1984, 48 (11), $2869-2870$. https://doi.org/10.1271/bbb1961.48.2869.

(6) Chen, E.; Song, H.; Li, Y.; Chen, H.; Wang, B.; Che, X.; Zhang, Y.; Zhao, S. Analysis of Aroma Components from Sugarcane to Non-Centrifugal Cane Sugar Using GC-O-MS. RSC Adv. 2020, 10 (54), 32276-32289. https://doi.org/10.1039/d0ra05963c.

(7) Wang, L.; Deng, W.; Wang, P.; Huang, W.; Wu, J.; Zheng, T.; Chen, J. Degradations of Aroma Characteristics and Changes of Aroma Related 
Compounds, PPO Activity, and Antioxidant Capacity in Sugarcane Juice during Thermal Process. J. Food Sci. 2020, 85 (4), $1140-1150$. https://doi.org/10.1111/1750-3841.15108.

(8) Asikin, Y.; Kamiya, A.; Mizu, M.; Takara, K.; Tamaki, H.; Wada, K. Changes in the Physicochemical Characteristics, Including Flavour Components and Maillard Reaction Products, of Non-Centrifugal Cane Brown Sugar during Storage. Food Chem. 2014, 149, $170-177$. https://doi.org/10.1016/j.foodchem.2013.10.089.

(9) Takahashi, M.; Ishmael, M.; Asikin, Y.; Hirose, N.; Mizu, M.; Shikanai, T.; Tamaki, H.; Wada, K. Composition, Taste, Aroma, and Antioxidant Activity of Solidified Noncentrifugal Brown Sugars Prepared from Whole Stalk and Separated Pith of Sugarcane (Saccharum Officinarum L.). J. Food Sci. 2016, 81 (11), C2647-C2655. https://doi.org/10.1111/1750-3841.13531.

(10) Asikin, Y.; Hirose, N.; Tamaki, H.; Ito, S.; Oku, H.; Wada, K. Effects of Different Drying-Solidification Processes on Physical Properties, Volatile Fraction, and Antioxidant Activity of Non-Centrifugal Cane Brown Sugar. LWT - Food Sci. Technol. 2016, 66, 340-347. https://doi.org/10.1016/j.lwt.2015.10.039.

(11) Payet, B.; Shum, A.; Sing, C.; Smadja, J. Assessment of Antioxidant Activity of Cane Brown Sugars by ABTS and DPPH Radical Scavenging Assays: Determination of Their Polyphenolic and Volatile Constituents. J. Agric. Food Chem. 2005, 53, 10074-10079. https://doi.org/10.1021/jf0517703.

(12) Edris, A. E.; Murkovic, M.; Siegmund, B. Application of Headspace-Solid-Phase Microextraction and HPLC for the Analysis of the Aroma Volatile Components of Treacle and Determination of Its Content of 5-Hydroxymethylfurfural (HMF). Food Chem. 2007, 104 (3), $1310-1314$. https://doi.org/10.1016/j.foodchem.2006.10.033.

(13) Quinn, B. P.; Bernier, U. R.; Geden, C. J.; Hogsette, J. A.; Carlson, D. A. Analysis of Extracted and Volatile Components in Blackstrap Molasses Feed as Candidate House Fly Attractants. J. Chromatogr. A 2007, 1139 (2), 279-284. https://doi.org/10.1016/j.chroma.2006.11.039.

(14) Franitza, L.; Granvogl, M.; Schieberle, P. Influence of the Production Process on the Key Aroma Compounds of Rum: From Molasses to the Spirit. J. Agric. Food Chem. 2016, 64 (47), 9041-9053. https://doi.org/10.1021/acs.jafc.6b04046.

(15) Abe, E.; Nakatani, Y.; Yamanishi, T.; Muraki, S. Studies on the" Sugary Flavor" of Raw Cane Sugar. I. Proc. Japan Acad. Ser. B Phys. Biol. Sci. 1978, 54 (9), 542-547. https://doi.org/10.2183/pjab.54.542.

(16) Asikin, Y.; Wada, K.; Imai, Y.; Kawamoto, Y.; Mizu, M.; Mutsuura, M.; Takahashi, M. Compositions, Taste Characteristics, Volatile Profiles, and Antioxidant Activities of Sweet Sorghum (Sorghum Bicolor L.) and Sugarcane (Saccharum Officinarum L.) Syrups. J. Food Meas. Charact. 2018, 12 (2), 884-891. https://doi.org/10.1007/s11694-017-9703-2.

(17) Ruiz-Matute, A. I.; Soria, A. C.; Sanz, M. L.; Martínez-Castro, I. Characterization of Traditional Spanish Edible Plant Syrups Based on Carbohydrate GC- 
MS Analysis. J. Food Compos. Anal. 2010, 23 (3), 260-263. https://doi.org/10.1016/j.jfca.2009.08.017.

(18) de Andrade, J. K.; Komatsu, E.; Perreault, H.; Torres, Y. R.; da Rosa, M. R.; Felsner, M. L. In House Validation from Direct Determination of 5Hydroxymethyl-2-Furfural (HMF) in Brazilian Corn and Cane Syrups Samples by HPLC-UV. Food Chem. 2016, 190, 481-486. https://doi.org/10.1016/j.foodchem.2015.05.131.

(19) Serafim, F. A. T.; Pereira-Filho, E. R.; Franco, D. W. Chemical Data as Markers of the Geographical Origins of Sugarcane Spirits. Food Chem. 2016, 196, 196-203. https://doi.org/10.1016/j.foodchem.2015.09.040.

(20) Coelho, C.; Brottier, C.; Beuchet, F.; Elichiry-Ortiz, P.; Bach, B.; Lafarge, C.; Tourdot-Maréchal, R. Effect of Ageing on Lees and Distillation Process on Fermented Sugarcane Molasses for the Production of Rum. Food Chem. 2020, 303. https://doi.org/10.1016/j.foodchem.2019.125405.

(21) Zacaroni, L. M.; de Sales, P. F.; Cardoso, M. das G.; Santiago, W. D.; Nelson, D. L. Response Surface Optimization of SPME Extraction Conditions for the Analysis of Volatile Compounds in Brazilian Sugar Cane Spirits by HS-SPME-GC-MS. J. Inst. Brew. 2017, 123 (2), $226-231$. https://doi.org/10.1002/jib.410.

(22) De Souza, M. D. C. A.; Vásquez, P.; Del Mastro, N. L.; Acree, T. E.; Lavin, E. H. Characterization of Cachaça and Rum Aroma. J. Agric. Food Chem. 2006, 54 (2), 485-488. https://doi.org/10.1021/jf0511190.

(23) Cardeal, Z. L.; Marriott, P. J. Comprehensive Two-Dimensional Gas Chromatography-Mass Spectrometry Analysis and Comparison of Volatile Organic Compounds in Brazilian Cachaça and Selected Spirits. Food Chem. 2009, 112 (3), 747-755. https://doi.org/10.1016/j.foodchem.2008.06.057.

(24) Franitza, L.; Nicolotti, L.; Granvog1, M.; Schieberle, P. Differentiation of Rums Produced from Sugar Cane Juice (Rhum Agricole) from Rums Manufactured from Sugar Cane Molasses by a Metabolomics Approach. J. Agric. Food Chem. 2018, 66 (11), 3038-3045. https://doi.org/10.1021/acs.jafc.8b00180.

(25) Tábua, M. C. M.; Santiago, W. D.; Magalhães, M. L.; Ferreira, V. R. F.; Brandão, R. M.; Teixeira, M. L.; Pedroso, M. P.; de Resende Machado, A. M.; Nelson, D. L.; das Graças Cardoso, M. Identification of Volatile Compounds, Quantification of Glycerol and Trace Elements in Distilled Spirits Produced in Mozambique. J. Food Sci. Technol. 2020, 57 (2), 505-512. https://doi.org/10.1007/s13197-019-04079-9.

(26) García, J. M.; Narváez, P. C.; Heredia, F. J.; Orjuela, Á.; Osorio, C. Physicochemical and Sensory (Aroma and Colour) Characterisation of a NonCentrifugal Cane Sugar ("Panela") Beverage. Food Chem. 2017, 228, 7-13. https://doi.org/10.1016/j.foodchem.2017.01.134.

(27) Resende Oliveira, É.; Caliari, M.; Soares Soares Júnior, M.; Ribeiro Oliveira, A.; Cristina Marques Duarte, R.; Valério de Barros Vilas Boas, E. Assessment of Chemical and Sensory Quality of Sugarcane Alcoholic Fermented Beverage. J. Food Sci. Technol. 2018,55 (1), $72-81$. https://doi.org/10.1007/s13197-017-2792-4. 
(28) Tzeng, D. I.; Chia, Y. C.; Tai, C. Y.; Ou, A. S. M. Investigation of Chemical Quality of Sugarcane (Saccharum Officinarum 1.) Wine during Fermentation by Saccharomyces Cerevisiae. J. Food Qual. 2010, 33 (2), 248-267. https://doi.org/10.1111/j.1745-4557.2010.00305.x.

Table S6. Summary of number of volatile organic compounds identified, relative peak areas and total relative peak areas (\%) values of main chemical classes identified in sugarcane samples.

\begin{tabular}{|c|c|c|c|c|c|c|c|c|c|c|c|c|c|c|c|c|c|c|c|c|}
\hline \multirow{3}{*}{$\begin{array}{c}\text { Main Chemical } \\
\text { Class }\end{array}$} & \multirow{3}{*}{ Abbreviation } & \multirow{3}{*}{$\begin{array}{l}\text { Harvest } \\
\text { Year }\end{array}$} & \multicolumn{18}{|c|}{ Sugarcane Varieties } \\
\hline & & & \multicolumn{3}{|c|}{ AMA } & \multicolumn{3}{|c|}{ RAD } & \multicolumn{3}{|c|}{ ROX } & \multicolumn{3}{|c|}{ VER } & \multicolumn{3}{|c|}{ VIO } & \multicolumn{3}{|c|}{ CAN } \\
\hline & & & $\mathbf{N}^{01}$ & $\mathbf{R P A}^{2}$ & $\begin{array}{c}\text { TRPA }^{3} \\
(\%)\end{array}$ & $N^{01}$ & $\mathbf{R P A}^{2}$ & $\begin{array}{c}\text { TRPA }^{3} \\
(\%)\end{array}$ & $N^{01}$ & $\mathbf{R P A}^{2}$ & $\begin{array}{c}\text { TRPA }^{3} \\
(\%)\end{array}$ & $N^{01}$ & $\mathbf{R P A}^{2}$ & $\begin{array}{c}\operatorname{TRPA}^{3} \\
(\%)\end{array}$ & $\mathbf{N}^{01}$ & $\mathbf{R P A}^{2}$ & $\begin{array}{c}\text { TRPA }^{3} \\
(\%)\end{array}$ & $\mathbf{N}^{01}$ & $\mathbf{R P A}^{2}$ & $\begin{array}{c}\text { TRPA }^{3} \\
(\%)\end{array}$ \\
\hline \multirow[t]{2}{*}{ Alcohol } & ALC & 2015 & 49 & 3665.7 & 31.2 & 49 & 4513.9 & 33.5 & 49 & 3860.5 & 36.1 & 49 & 3604.2 & 28.8 & 49 & 4264.6 & 45.6 & 49 & 2044.7 & 21.6 \\
\hline & & 2017 & 49 & 4496.0 & 33.5 & 49 & 4904.7 & 34.8 & 49 & 3919.4 & 35.7 & 49 & 4064.3 & 30.2 & 49 & 5265.1 & 49.4 & 49 & 1971.6 & 21.2 \\
\hline \multirow[t]{2}{*}{ Aldehyde } & ALD & 2015 & 19 & 422.2 & 3.6 & 19 & 415.9 & 3.1 & 19 & 545.4 & 5.1 & 19 & 555.7 & 4.4 & 19 & 204.8 & 2.2 & 19 & 486.7 & 5.1 \\
\hline & & 2017 & 19 & 521.1 & 3.9 & 19 & 413.6 & 2.9 & 19 & 467.3 & 4.3 & 19 & 536.3 & 4.0 & 19 & 214.9 & 2.0 & 19 & 424.3 & 4.6 \\
\hline \multirow[t]{2}{*}{ Benzene } & BNZ & 2015 & 33 & 701.4 & 6.0 & 33 & 497.2 & 3.7 & 33 & 952.3 & 8.9 & 33 & 981.7 & 7.8 & 33 & 882.5 & 9.4 & 33 & 582.5 & 6.2 \\
\hline & & 2017 & 33 & 788.7 & 5.9 & 33 & 548.6 & 3.9 & 33 & 982.2 & 9.0 & 33 & 1120.1 & 8.3 & 33 & 1027.2 & 9.6 & 33 & 589.4 & 6.3 \\
\hline \multirow[t]{2}{*}{ Carboxylic Acid } & CAC & 2015 & 10 & 2113.8 & 18.0 & 10 & 2030.3 & 15.1 & 10 & 1630.1 & 15.2 & 10 & 2091.9 & 16.7 & 10 & 544.2 & 5.8 & 10 & 332.3 & 3.5 \\
\hline & & 2017 & 10 & 2509.2 & 18.7 & 10 & 2444.2 & 17.3 & 10 & 1797.4 & 16.4 & 10 & 2334.4 & 17.3 & 10 & 558.4 & 5.2 & 10 & 318.5 & 3.4 \\
\hline \multirow[t]{2}{*}{ Ester } & EST & 2015 & 14 & 202.4 & 1.7 & 14 & 178.0 & 1.3 & 14 & 921.2 & 8.6 & 14 & 280.2 & 2.2 & 14 & 156.1 & 1.7 & 14 & 167.8 & 1.8 \\
\hline & & 2017 & 14 & 262.8 & 2.0 & 14 & 218.7 & 1.6 & 14 & 1306.6 & 11.9 & 14 & 343.8 & 2.6 & 14 & 158.4 & 1.5 & 14 & 162.6 & 1.8 \\
\hline Ether & & 2017 & 6 & 115.3 & 0.9 & 6 & 66.6 & 0.5 & 6 & 94.5 & 0.9 & 6 & 120.0 & 0.9 & 6 & 61.5 & 0.6 & 6 & 374.4 & 4.0 \\
\hline \multirow[t]{2}{*}{ Furan } & FUR & 2015 & 10 & 554.2 & 4.7 & 10 & 759.1 & 5.6 & 10 & 284.6 & 2.7 & 10 & 282.9 & 2.3 & 10 & 203.3 & 2.2 & 10 & 162.1 & 1.7 \\
\hline & & 2017 & 10 & 631.7 & 4.7 & 10 & 746.7 & 5.3 & 10 & 283.3 & 2.6 & 10 & 324.4 & 2.4 & 10 & 213.1 & 2.0 & 10 & 165.2 & 1.8 \\
\hline \multirow[t]{2}{*}{ Hydrocarbon } & HYD & 2015 & 38 & 555.7 & 4.7 & 38 & 747.6 & 5.5 & 38 & 485.4 & 4.5 & 38 & 854.7 & 6.8 & 38 & 702.8 & 7.5 & 38 & 1181.7 & 12.5 \\
\hline & & 2017 & 38 & 602.1 & 4.5 & 38 & 653.9 & 4.6 & 38 & 384.5 & 3.5 & 38 & 549.7 & 4.1 & 38 & 615.5 & 5.8 & 38 & 1371.2 & 14.8 \\
\hline \multirow[t]{2}{*}{ Ketone } & KET & 2015 & 39 & 1780.6 & 15.2 & 39 & 3034.6 & 22.5 & 39 & 960.0 & 9.0 & 39 & 2102.8 & 16.8 & 39 & 1349.7 & 14.4 & 39 & 2447.5 & 25.9 \\
\hline & & 2017 & 39 & 1769.0 & 13.2 & 39 & 3016.8 & 21.4 & 39 & 855.9 & 7.8 & 39 & 2251.7 & 16.7 & 39 & 1479.0 & 13.9 & 39 & 2357.0 & 25.4 \\
\hline Naphthalene & NPH & 2015 & 1 & 16.4 & 0.1 & 1 & 7.5 & 0.1 & 1 & 19.1 & 0.2 & 1 & 13.2 & 0.1 & 1 & 10.7 & 0.1 & 1 & 1.3 & 0.0 \\
\hline
\end{tabular}




\begin{tabular}{|c|c|c|c|c|c|c|c|c|c|c|c|c|c|c|c|c|c|c|c|c|}
\hline & & 2017 & 1 & 17.0 & 0.1 & 1 & 8.3 & 0.1 & 1 & 20.4 & 0.2 & 1 & 16.0 & 0.1 & 1 & 9.0 & 0.1 & 1 & 1.4 & 0.0 \\
\hline \multirow[t]{2}{*}{ Nitrogen } & NIT & 2015 & 5 & 180.2 & 1.5 & 5 & 56.8 & 0.4 & 5 & 81.6 & 0.8 & 5 & 126.4 & 1.0 & 5 & 46.5 & 0.5 & 5 & 192.5 & 2.0 \\
\hline & & 2017 & 5 & 209.1 & 1.6 & 5 & 64.0 & 0.5 & 5 & 80.7 & 0.7 & 5 & 137.2 & 1.0 & 5 & 40.4 & 0.4 & 5 & 196.9 & 2.1 \\
\hline \multirow[t]{2}{*}{ Phenol } & PHE & 2015 & 7 & 228.0 & 1.9 & 7 & 150.2 & 1.1 & 7 & 223.4 & 2.1 & 7 & 299.8 & 2.4 & 7 & 198.4 & 2.1 & 7 & 81.7 & 0.9 \\
\hline & & 2017 & 7 & 249.9 & 1.9 & 7 & 168.8 & 1.2 & 7 & 211.5 & 1.9 & 7 & 346.2 & 2.6 & 7 & 216.8 & 2.0 & 7 & 84.8 & 0.9 \\
\hline \multirow[t]{2}{*}{ Pyran } & PYR & 2015 & 3 & 192.1 & 1.6 & 3 & 218.6 & 1.6 & 3 & 149.4 & 1.4 & 3 & 243.7 & 1.9 & 3 & 5.3 & 0.1 & 3 & 129.2 & 1.4 \\
\hline & & 2017 & 3 & 252.8 & 1.9 & 3 & 260.7 & 1.8 & 3 & 134.4 & 1.2 & 3 & 294.3 & 2.2 & 3 & 25.7 & 0.2 & 3 & 121.9 & 1.3 \\
\hline \multirow[t]{2}{*}{ Sulfur } & SUL & 2015 & 3 & 850.9 & 7.2 & 3 & 619.5 & 4.6 & 3 & 351.2 & 3.3 & 3 & 592.4 & 4.7 & 3 & 547.5 & 5.9 & 3 & 242.0 & 2.6 \\
\hline & & 2017 & 3 & 879.1 & 6.5 & 3 & 457.7 & 3.2 & 3 & 325.9 & 3.0 & 3 & 698.5 & 5.2 & 3 & 678.3 & 6.4 & 3 & 307.5 & 3.3 \\
\hline \multirow{2}{*}{$\begin{array}{l}\text { Terpene / } \\
\text { Terpenoid }\end{array}$} & TER & 2015 & 23 & 108.0 & 0.9 & 23 & 111.8 & 0.8 & 23 & 102.9 & 1.0 & 23 & 240.8 & 1.9 & 23 & 93.1 & 1.0 & 23 & 1094.1 & 11.6 \\
\hline & & 2017 & 23 & 122.5 & 0.9 & 23 & 125.7 & 0.9 & 23 & 100.1 & 0.9 & 23 & 329.8 & 2.4 & 23 & 95.8 & 0.9 & 23 & 842.3 & 9.1 \\
\hline
\end{tabular}

${ }^{1}$ Number of volatile organic compounds identified.

${ }^{2}$ Relative Peak Areas values.

${ }^{3}$ Total Relative Peak Areas percentage values.

Table S7. One-way ANOVA with Post-hoc Tukey test results based on the relative peak areas of the identified VOCs in sugarcane samples.

\begin{tabular}{|c|c|c|c|c|c|c|c|c|}
\hline \multirow{3}{*}{ Volatile Organic Compounds } & \multicolumn{8}{|c|}{ One-way ANOVA Test } \\
\hline & \multicolumn{2}{|c|}{ All Samples } & \multicolumn{6}{|c|}{ Tukey Test $^{3}(\alpha<0.05)$} \\
\hline & $F^{1}$ & $\mathbf{P}^{2}$ & AMA (A) & RAD (B) & $\operatorname{ROX}(C)$ & VER (D) & VIO (E) & CAN (F) \\
\hline Pentane & 48.39 & $\leq 1.00 \mathrm{E}-07$ & $\mathrm{~B} ; \mathrm{C} ; \mathrm{E}$ & $\mathrm{A} ; \mathrm{C} ; \mathrm{D} ; \mathrm{F}$ & $\mathrm{A} ; \mathrm{B} ; \mathrm{D} ; \mathrm{E} ; \mathrm{F}$ & $\mathrm{B} ; \mathrm{C}$ & $\mathrm{A} ; \mathrm{C}$ & $\mathrm{B} ; \mathrm{C}$ \\
\hline 1-Pentene & 30.91 & $\leq 1.00 \mathrm{E}-07$ & $\mathrm{C} ; \mathrm{E} ; \mathrm{F}$ & $\mathrm{C} ; \mathrm{E} ; \mathrm{F}$ & $\mathrm{A} ; \mathrm{B} ; \mathrm{D} ; \mathrm{E}$ & $\mathrm{C} ; \mathrm{E}$ & $\mathrm{A} ; \mathrm{B} ; \mathrm{C} ; \mathrm{F}$ & A; B; D; E \\
\hline 2-Methyl-Pentane & 43.08 & $\leq 1.00 \mathrm{E}-07$ & $\mathrm{C} ; \mathrm{D}$ & $\mathrm{C} ; \mathrm{D} ; \mathrm{F}$ & $\mathrm{A} ; \mathrm{B} ; \mathrm{D} ; \mathrm{E} ; \mathrm{F}$ & $\mathrm{A} ; \mathrm{B} ; \mathrm{C} ; \mathrm{E} ; \mathrm{F}$ & $\mathrm{C} ; \mathrm{D}$ & $\mathrm{B} ; \mathrm{C} ; \mathrm{D}$ \\
\hline Hexane & 37.75 & $\leq 1.00 \mathrm{E}-07$ & $\mathrm{~F}$ & $\mathrm{~F}$ & $\mathrm{~F}$ & $\mathrm{~F}$ & $\mathrm{~F}$ & $\mathrm{~A} ; \mathrm{B} ; \mathrm{C} ; \mathrm{D} ; \mathrm{E}$ \\
\hline Ethyl Ether & 4.64 & $2.96 \mathrm{E}-03$ & $\mathrm{D}$ & & $\mathrm{F}$ & $\mathrm{A} ; \mathrm{F}$ & & $\mathrm{C} ; \mathrm{D}$ \\
\hline cis-4-Methyl-2-Pentene & 5.00 & $1.90 \mathrm{E}-03$ & $\mathrm{C}$ & $\mathrm{C}$ & $\mathrm{A} ; \mathrm{B} ; \mathrm{E} ; \mathrm{F}$ & & $\mathrm{C}$ & $\mathrm{C}$ \\
\hline cis-Piperylene & 27.39 & $\leq 1.00 \mathrm{E}-07$ & $\mathrm{C} ; \mathrm{D}$ & $\mathrm{C}$ & $\mathrm{A} ; \mathrm{B} ; \mathrm{D} ; \mathrm{E} ; \mathrm{F}$ & $\mathrm{A} ; \mathrm{C} ; \mathrm{E} ; \mathrm{F}$ & $\mathrm{C} ; \mathrm{D}$ & $\mathrm{C} ; \mathrm{D}$ \\
\hline 2-Methyl-1-Pentene & 4.53 & $3.40 \mathrm{E}-03$ & $\mathrm{~F}$ & & $\mathrm{~F}$ & & $\mathrm{~F}$ & $\mathrm{~A} ; \mathrm{C} ; \mathrm{E}$ \\
\hline
\end{tabular}




\begin{tabular}{|c|c|c|c|c|c|c|c|c|}
\hline cis-3-Methyl-2-Pentene & 30.80 & $\leq 1.00 \mathrm{E}-07$ & $\mathrm{~B} ; \mathrm{D}$ & $\mathrm{A} ; \mathrm{C} ; \mathrm{D} ; \mathrm{E} ; \mathrm{F}$ & $\mathrm{B} ; \mathrm{E}$ & $\mathrm{A} ; \mathrm{B} ; \mathrm{C}$ & $\mathrm{B}$ & B \\
\hline 3-Methyl-Hexane & 106.12 & $\leq 1.00 \mathrm{E}-07$ & $C ; D, E ; F$ & $\mathrm{E} ; \mathrm{F}$ & $\mathrm{A} ; \mathrm{F}$ & $\mathrm{A} ; \mathrm{E} ; \mathrm{F}$ & $\mathrm{A} ; \mathrm{B} ; \mathrm{D} ; \mathrm{F}$ & $\mathrm{A} ; \mathrm{B} ; \mathrm{C} ; \mathrm{D} ; \mathrm{E}$ \\
\hline 1,4-Pentadiene & 5.68 & $8.45 \mathrm{E}-04$ & $\mathrm{C}$ & & $\mathrm{A} ; \mathrm{D} ; \mathrm{E} ; \mathrm{F}$ & $\mathrm{C}$ & $\mathrm{C}$ & $\mathrm{C}$ \\
\hline Methanethiol & 65.91 & $\leq 1.00 \mathrm{E}-07$ & $\mathrm{~B} ; \mathrm{C} ; \mathrm{E}$ & $\mathrm{A} ; \mathrm{C} ; \mathrm{E} ; \mathrm{F}$ & $\mathrm{A} ; \mathrm{B} ; \mathrm{D} ; \mathrm{E} ; \mathrm{F}$ & $\mathrm{C} ; \mathrm{E}$ & $\mathrm{A} ; \mathrm{B} ; \mathrm{C} ; \mathrm{D} ; \mathrm{F}$ & $\mathrm{B} ; \mathrm{C} ; \mathrm{E}$ \\
\hline trans-Piperylene & 16.68 & $\leq 1.00 \mathrm{E}-07$ & $\mathrm{D} ; \mathrm{E}$ & $\mathrm{C}$ & $\mathrm{B} ; \mathrm{D} ; \mathrm{E} ; \mathrm{F}$ & $\mathrm{A} ; \mathrm{C}$ & $\mathrm{A} ; \mathrm{C}$ & $\mathrm{C}$ \\
\hline Heptane & 46.55 & $\leq 1.00 \mathrm{E}-07$ & $\mathrm{~B} ; \mathrm{C} ; \mathrm{D} ; \mathrm{F}$ & $\mathrm{A} ; \mathrm{C} ; \mathrm{D} ; \mathrm{F}$ & $\mathrm{A}, \mathrm{B} ; \mathrm{D} ; \mathrm{E}$ & $\mathrm{A} ; \mathrm{B} ; \mathrm{C} ; \mathrm{E} ; \mathrm{F}$ & $\mathrm{C} ; \mathrm{D} ; \mathrm{F}$ & $\mathrm{A} ; \mathrm{B} ; \mathrm{D} ; \mathrm{E}$ \\
\hline tert-Butyl Ethyl Ether & 26.94 & $\leq 1.00 \mathrm{E}-07$ & $\mathrm{~F}$ & $\mathrm{~F}$ & $\mathrm{~F}$ & $\mathrm{~F}$ & $\mathrm{~F}$ & $\mathrm{~A} ; \mathrm{B} ; \mathrm{C} ; \mathrm{D} ; \mathrm{E}$ \\
\hline Ethanal & 55.79 & $\leq 1.00 \mathrm{E}-07$ & $\mathrm{~B} ; \mathrm{C} ; \mathrm{E}$ & $\mathrm{A} ; \mathrm{D} ; \mathrm{E} ; \mathrm{F}$ & $\mathrm{A} ; \mathrm{D} ; \mathrm{E} ; \mathrm{F}$ & $\mathrm{B} ; \mathrm{C} ; \mathrm{D}$ & $\mathrm{A} ; \mathrm{B} ; \mathrm{C} ; \mathrm{D} ; \mathrm{F}$ & $\mathrm{B} ; \mathrm{C} ; \mathrm{E}$ \\
\hline Dimethyl Sulfide & 61.07 & $\leq 1.00 \mathrm{E}-07$ & $\mathrm{~B} ; \mathrm{C} ; \mathrm{D} ; \mathrm{E} ; \mathrm{F}$ & $\mathrm{A} ; \mathrm{C}$ & $\mathrm{A} ; \mathrm{B} ; \mathrm{D} ; \mathrm{E}$ & $\mathrm{A} ; \mathrm{C} ; \mathrm{F}$ & $\mathrm{A} ; \mathrm{C} ; \mathrm{F}$ & $\mathrm{A} ; \mathrm{B} ; \mathrm{D} ; \mathrm{E}$ \\
\hline 4-Methyl-Heptane & 22.08 & $\leq 1.00 \mathrm{E}-07$ & $\mathrm{~F}$ & $\mathrm{~F}$ & $\mathrm{~F}$ & $\mathrm{~F}$ & $\mathrm{~F}$ & $\mathrm{~A} ; \mathrm{B} ; \mathrm{C} ; \mathrm{D} ; \mathrm{E}$ \\
\hline Octane & 92.54 & $\leq 1.00 \mathrm{E}-07$ & $\mathrm{~B} ; \mathrm{C} ; \mathrm{D} ; \mathrm{E} ; \mathrm{F}$ & $\mathrm{A} ; \mathrm{C} ; \mathrm{D} ; \mathrm{E} ; \mathrm{F}$ & $\mathrm{A} ; \mathrm{B} ; \mathrm{D} ; \mathrm{E}$ & $\mathrm{A}, \mathrm{B} ; \mathrm{C} ; \mathrm{F}$ & $\mathrm{A} ; \mathrm{B} ; \mathrm{C} ; \mathrm{F}$ & $\mathrm{A} ; \mathrm{B} ; \mathrm{D} ; \mathrm{E}$ \\
\hline Propanal & 35.24 & $\leq 1.00 \mathrm{E}-07$ & $\mathrm{E} ; \mathrm{F}$ & $\mathrm{F}$ & $\mathrm{E} ; \mathrm{F}$ & $\mathrm{F}$ & $\mathrm{A} ; \mathrm{C} ; \mathrm{F}$ & $\mathrm{A} ; \mathrm{B} ; \mathrm{C} ; \mathrm{D} ; \mathrm{E}$ \\
\hline Furan & 18.66 & $\leq 1.00 \mathrm{E}-07$ & $\mathrm{D} ; \mathrm{E} ; \mathrm{F}$ & $\mathrm{E} ; \mathrm{F}$ & $\mathrm{E} ; \mathrm{F}$ & $\mathrm{A} ; \mathrm{E} ; \mathrm{F}$ & $\mathrm{A} ; \mathrm{B} ; \mathrm{C} ; \mathrm{D}$ & $\mathrm{A} ; \mathrm{B} ; \mathrm{C} ; \mathrm{D}$ \\
\hline 2,4-Dimethyl-Heptane & 11.84 & $2.00 \mathrm{E}-06$ & $\mathrm{C}$ & $\mathrm{C} ; \mathrm{F}$ & $\mathrm{A} ; \mathrm{B} ; \mathrm{D} ; \mathrm{E} ; \mathrm{F}$ & $\mathrm{C} ; \mathrm{F}$ & $\mathrm{C}$ & $\mathrm{B} ; \mathrm{C} ; \mathrm{D}$ \\
\hline 2-Methyl-Propanal & 165.43 & $\leq 1.00 \mathrm{E}-07$ & $\mathrm{~B} ; \mathrm{C} ; \mathrm{D}$ & $\mathrm{A} ; \mathrm{C} ; \mathrm{D} ; \mathrm{E} ; \mathrm{F}$ & $\mathrm{A} ; \mathrm{B} ; \mathrm{D} ; \mathrm{E} ; \mathrm{F}$ & $\mathrm{A} ; \mathrm{B} ; \mathrm{C} ; \mathrm{E} ; \mathrm{F}$ & $\mathrm{B} ; \mathrm{C} ; \mathrm{D}$ & $\mathrm{B} ; \mathrm{C} ; \mathrm{D}$ \\
\hline 2-Propanone & 214.47 & $\leq 1.00 \mathrm{E}-07$ & $\mathrm{~B} ; \mathrm{C} ; \mathrm{D} ; \mathrm{E} ; \mathrm{F}$ & $\mathrm{A} ; \mathrm{C} ; \mathrm{E} ; \mathrm{F}$ & $\mathrm{A} ; \mathrm{B} ; \mathrm{C} ; \mathrm{F}$ & $\mathrm{A} ; \mathrm{C} ; \mathrm{E} ; \mathrm{F}$ & $\mathrm{A} ; \mathrm{B} ; \mathrm{D} ; \mathrm{F}$ & $\mathrm{A} ; \mathrm{B} ; \mathrm{C} ; \mathrm{D} ; \mathrm{E}$ \\
\hline Methyl Acetate & 5.30 & $1.32 \mathrm{E}-03$ & $\mathrm{C}$ & $\mathrm{C}$ & $\mathrm{A} ; \mathrm{B} ; \mathrm{E} ; \mathrm{F}$ & & $\mathrm{C}$ & $\mathrm{C}$ \\
\hline 2-Methyl-2-Heptene & 155.28 & $\leq 1.00 \mathrm{E}-07$ & $\mathrm{~F}$ & $\mathrm{~F}$ & $\mathrm{~F}$ & $\mathrm{~F}$ & $\mathrm{~F}$ & $\mathrm{~A} ; \mathrm{B} ; \mathrm{C} ; \mathrm{D} ; \mathrm{E}$ \\
\hline Crotonal & 24.32 & $\leq 1.00 \mathrm{E}-07$ & $\mathrm{~B} ; \mathrm{C} ; \mathrm{D} ; \mathrm{E}$ & $\mathrm{A} ; \mathrm{D} ; \mathrm{E}$ & $\mathrm{A} ; \mathrm{F}$ & $\mathrm{A} ; \mathrm{B} ; \mathrm{F}$ & $\mathrm{A} ; \mathrm{B} ; \mathrm{F}$ & $\mathrm{C} ; \mathrm{D} ; \mathrm{E}$ \\
\hline 2,3-Dimethyl-Heptane & 11.25 & $4.00 \mathrm{E}-06$ & $\mathrm{C}$ & $\mathrm{C}$ & $\mathrm{A} ; \mathrm{B} ; \mathrm{E} ; \mathrm{F}$ & $\mathrm{E} ; \mathrm{F}$ & $\mathrm{C} ; \mathrm{D}$ & $C ; D$ \\
\hline 4-Methyl-Octane & 102.07 & $\leq 1.00 \mathrm{E}-07$ & $\mathrm{C} ; \mathrm{F}$ & $\mathrm{C}$ & A; B; D; E; F & $\mathrm{C} ; \mathrm{F}$ & $\mathrm{C} ; \mathrm{F}$ & $\mathrm{A} ; \mathrm{C} ; \mathrm{D} ; \mathrm{E}$ \\
\hline 2-Methyl-Furan & 29.72 & $\leq 1.00 \mathrm{E}-07$ & $\mathrm{~B} ; \mathrm{C}$ & $\mathrm{A} ; \mathrm{C} ; \mathrm{D} ; \mathrm{E} ; \mathrm{F}$ & $\mathrm{A} ; \mathrm{B}$ & $\mathrm{B}$ & $\mathrm{B}$ & $\mathrm{B}$ \\
\hline 2,4-Dimethyl-1-Heptene & 324.27 & $\leq 1.00 \mathrm{E}-07$ & $\mathrm{~F}$ & $\mathrm{~F}$ & $\mathrm{~F}$ & $\mathrm{~F}$ & $\mathrm{~F}$ & $\mathrm{~A} ; \mathrm{B} ; \mathrm{C} ; \mathrm{D} ; \mathrm{E}$ \\
\hline Ethyl Acetate & 217.48 & $\leq 1.00 \mathrm{E}-07$ & $\mathrm{C}$ & $\mathrm{C}$ & A; B; D; E; F & $\mathrm{C} ; \mathrm{E}$ & $\mathrm{C} ; \mathrm{D}$ & $\mathrm{C}$ \\
\hline Nonane & 31.19 & $\leq 1.00 \mathrm{E}-07$ & $\mathrm{~B} ; \mathrm{C} ; \mathrm{E} ; \mathrm{F}$ & $\mathrm{A} ; \mathrm{C} ; \mathrm{D}$ & $\mathrm{A} ; \mathrm{B} ; \mathrm{D} ; \mathrm{E}$ & $\mathrm{B} ; \mathrm{C} ; \mathrm{F}$ & $\mathrm{A} ; \mathrm{C} ; \mathrm{F}$ & $\mathrm{A} ; \mathrm{B} ; \mathrm{D} ; \mathrm{E}$ \\
\hline 2-Butanone & 143.17 & $\leq 1.00 \mathrm{E}-07$ & $\mathrm{~B} ; \mathrm{C} ; \mathrm{D} ; \mathrm{E} ; \mathrm{F}$ & $\mathrm{A} ; \mathrm{C} ; \mathrm{D} ; \mathrm{F}$ & $\mathrm{A} ; \mathrm{B} ; \mathrm{E} ; \mathrm{F}$ & $\mathrm{A} ; \mathrm{B} ; \mathrm{E} ; \mathrm{F}$ & $\mathrm{A} ; \mathrm{C} ; \mathrm{D} ; \mathrm{F}$ & $\mathrm{A} ; \mathrm{B} ; \mathrm{C} ; \mathrm{D} ; \mathrm{E}$ \\
\hline 2-Methyl-Butanal & 81.36 & $\leq 1.00 \mathrm{E}-07$ & $\mathrm{~B} ; \mathrm{C} ; \mathrm{E} ; \mathrm{F}$ & $\mathrm{A} ; \mathrm{D} ; \mathrm{E}$ & $\mathrm{A} ; \mathrm{D} ; \mathrm{E}$ & $\mathrm{B} ; \mathrm{C} ; \mathrm{E}$ & $\mathrm{A} ; \mathrm{B} ; \mathrm{C} ; \mathrm{D} ; \mathrm{F}$ & $\mathrm{A} ; \mathrm{D} ; \mathrm{E}$ \\
\hline 3-Methyl-Butanal & 33.25 & $\leq 1.00 \mathrm{E}-07$ & $\mathrm{~B} ; \mathrm{C} ; \mathrm{E}$ & A; D; E; F & $\mathrm{A} ; \mathrm{D} ; \mathrm{E} ; \mathrm{F}$ & $\mathrm{B} ; \mathrm{C} ; \mathrm{E}$ & $\mathrm{A} ; \mathrm{B} ; \mathrm{C} ; \mathrm{D} ; \mathrm{F}$ & $\mathrm{B} ; \mathrm{C} ; \mathrm{E}$ \\
\hline Ethyl Alcohol & 112.86 & $\leq 1.00 \mathrm{E}-07$ & $\mathrm{~B} ; \mathrm{C} ; \mathrm{D} ; \mathrm{E} ; \mathrm{F}$ & $\mathrm{A} ; \mathrm{C} ; \mathrm{D} ; \mathrm{F}$ & A; B; D; E; F & $\mathrm{A} ; \mathrm{B} ; \mathrm{C} ; \mathrm{E} ; \mathrm{F}$ & $\mathrm{A} ; \mathrm{C} ; \mathrm{D} ; \mathrm{F}$ & $\mathrm{A} ; \mathrm{B} ; \mathrm{C} ; \mathrm{D} ; \mathrm{E}$ \\
\hline Benzene & 27.31 & $\leq 1.00 \mathrm{E}-07$ & $\mathrm{~B} ; \mathrm{C} ; \mathrm{D} ; \mathrm{E}$ & $\mathrm{A} ; \mathrm{C} ; \mathrm{F}$ & $\mathrm{A} ; \mathrm{B} ; \mathrm{E} ; \mathrm{F}$ & $\mathrm{A} ; \mathrm{E} ; \mathrm{F}$ & $\mathrm{A} ; \mathrm{C} ; \mathrm{D}$ & $\mathrm{B} ; \mathrm{C} ; \mathrm{D}$ \\
\hline
\end{tabular}




\begin{tabular}{|c|c|c|c|c|c|c|c|c|}
\hline 3-Ethyl-Heptane & 97.26 & $\leq 1.00 \mathrm{E}-07$ & $\mathrm{~B} ; \mathrm{D} ; \mathrm{E} ; \mathrm{F}$ & $\mathrm{A} ; \mathrm{C} ; \mathrm{F}$ & $\mathrm{B} ; \mathrm{D} ; \mathrm{E} ; \mathrm{F}$ & $\mathrm{A} ; \mathrm{C} ; \mathrm{F}$ & $\mathrm{A} ; \mathrm{C} ; \mathrm{F}$ & $\mathrm{A} ; \mathrm{B} ; \mathrm{C} ; \mathrm{D} ; \mathrm{E}$ \\
\hline 2-Ethyl-Furan & 5.61 & $9.20 \mathrm{E}-04$ & $\mathrm{~B} ; \mathrm{C} ; \mathrm{D}$ & A & A & A & & \\
\hline Ethyl Propanoate & 76.40 & $\leq 1.00 \mathrm{E}-07$ & $\mathrm{C}$ & $\mathrm{C}$ & $\mathrm{A} ; \mathrm{B} ; \mathrm{D} ; \mathrm{E} ; \mathrm{F}$ & $\mathrm{C}$ & $\mathrm{C}$ & $\mathrm{C}$ \\
\hline Ethyl 2-Methylpropanoate & 115.73 & $\leq 1.00 \mathrm{E}-07$ & $\mathrm{C}$ & $\mathrm{C}$ & $\mathrm{A} ; \mathrm{B} ; \mathrm{D} ; \mathrm{E} ; \mathrm{F}$ & $\mathrm{C}$ & $\mathrm{C}$ & $\mathrm{C}$ \\
\hline 2-Methyl-Crotonal & 350.13 & $\leq 1.00 \mathrm{E}-07$ & $\mathrm{~B} ; \mathrm{C} ; \mathrm{D} ; \mathrm{E} ; \mathrm{F}$ & $\mathrm{A} ; \mathrm{C} ; \mathrm{D} ; \mathrm{E}$ & $\mathrm{A} ; \mathrm{B} ; \mathrm{D} ; \mathrm{E} ; \mathrm{F}$ & $\mathrm{A} ; \mathrm{B} ; \mathrm{C} ; \mathrm{E} ; \mathrm{F}$ & $\mathrm{A} ; \mathrm{B} ; \mathrm{C} ; \mathrm{D} ; \mathrm{F}$ & $\mathrm{A} ; \mathrm{C} ; \mathrm{D} ; \mathrm{E}$ \\
\hline Propyl Acetate & 77.02 & $\leq 1.00 \mathrm{E}-07$ & $\mathrm{C}$ & $\mathrm{C}$ & $\mathrm{A} ; \mathrm{B} ; \mathrm{D} ; \mathrm{E} ; \mathrm{F}$ & $\mathrm{C}$ & $\mathrm{C}$ & $\mathrm{C}$ \\
\hline 3-Pentanone & 53.64 & $\leq 1.00 \mathrm{E}-07$ & $\mathrm{~B} ; \mathrm{C} ; \mathrm{D} ; \mathrm{F}$ & $\mathrm{A} ; \mathrm{E} ; \mathrm{F}$ & $\mathrm{A} ; \mathrm{D} ; \mathrm{E} ; \mathrm{F}$ & $\mathrm{A} ; \mathrm{C} ; \mathrm{E} ; \mathrm{F}$ & $\mathrm{B} ; \mathrm{C} ; \mathrm{D} ; \mathrm{F}$ & $\mathrm{A} ; \mathrm{B} ; \mathrm{C} ; \mathrm{D} ; \mathrm{E}$ \\
\hline Pentanal & 288.09 & $\leq 1.00 \mathrm{E}-07$ & $\mathrm{C} ; \mathrm{F}$ & $\mathrm{C} ; \mathrm{F}$ & $\mathrm{A} ; \mathrm{B} ; \mathrm{D} ; \mathrm{E} ; \mathrm{F}$ & $\mathrm{C} ; \mathrm{F}$ & $\mathrm{C} ; \mathrm{F}$ & $\mathrm{A} ; \mathrm{B} ; \mathrm{C} ; \mathrm{D} ; \mathrm{E}$ \\
\hline 2,3-Butanedione & 134.31 & $\leq 1.00 \mathrm{E}-07$ & $\mathrm{~B} ; \mathrm{C} ; \mathrm{D} ; \mathrm{E} ; \mathrm{F}$ & $\mathrm{A} ; \mathrm{E} ; \mathrm{F}$ & $\mathrm{A} ; \mathrm{E} ; \mathrm{F}$ & $\mathrm{A} ; \mathrm{E} ; \mathrm{F}$ & $\mathrm{A} ; \mathrm{B} ; \mathrm{C} ; \mathrm{D} ; \mathrm{F}$ & $\mathrm{A} ; \mathrm{B} ; \mathrm{C} ; \mathrm{D} ; \mathrm{E}$ \\
\hline 2,3,4-Trimethyl-Hexane & 285.58 & $\leq 1.00 \mathrm{E}-07$ & $\mathrm{~F}$ & $\mathrm{~F}$ & $\mathrm{~F}$ & $\mathrm{~F}$ & $\mathrm{~F}$ & $\mathrm{~A} ; \mathrm{B} ; \mathrm{C} ; \mathrm{D} ; \mathrm{E}$ \\
\hline Decane & 59.20 & $\leq 1.00 \mathrm{E}-07$ & $\mathrm{E} ; \mathrm{F}$ & $\mathrm{E} ; \mathrm{F}$ & $\mathrm{F}$ & $\mathrm{F}$ & $\mathrm{A} ; \mathrm{B} ; \mathrm{F}$ & $\mathrm{A} ; \mathrm{B} ; \mathrm{C} ; \mathrm{D} ; \mathrm{E}$ \\
\hline 2,6-Dimethyl-Nonane & 92.65 & $\leq 1.00 \mathrm{E}-07$ & $\mathrm{~F}$ & $\mathrm{~F}$ & $\mathrm{~F}$ & $\mathrm{~F}$ & $\mathrm{~F}$ & $\mathrm{~A} ; \mathrm{B} ; \mathrm{C} ; \mathrm{D} ; \mathrm{E}$ \\
\hline 2,5-Dimethyl-Nonane & 216.67 & $\leq 1.00 \mathrm{E}-07$ & $\mathrm{C} ; \mathrm{E}$ & $\mathrm{C} ; \mathrm{F}$ & $\mathrm{A} ; \mathrm{B} ; \mathrm{D} ; \mathrm{E} ; \mathrm{F}$ & $\mathrm{C} ; \mathrm{F}$ & $\mathrm{A} ; \mathrm{C} ; \mathrm{F}$ & $\mathrm{B} ; \mathrm{C} ; \mathrm{D} ; \mathrm{E}$ \\
\hline 4-Methyl-2-Pentanone & 159.02 & $\leq 1.00 \mathrm{E}-07$ & $\mathrm{~F}$ & $\mathrm{~F}$ & $\mathrm{~F}$ & $\mathrm{~F}$ & $\mathrm{~F}$ & $\mathrm{~A} ; \mathrm{B} ; \mathrm{C} ; \mathrm{D} ; \mathrm{E}$ \\
\hline 2-Methy-2-Butanol & 342.89 & $\leq 1.00 \mathrm{E}-07$ & $\mathrm{~F}$ & $\mathrm{~F}$ & $\mathrm{~F}$ & $\mathrm{~F}$ & $\mathrm{~F}$ & $\mathrm{~A} ; \mathrm{B} ; \mathrm{C} ; \mathrm{D} ; \mathrm{E}$ \\
\hline 2-Methylpropyl Acetate & 33.42 & $\leq 1.00 \mathrm{E}-07$ & $\mathrm{C}$ & $\mathrm{C}$ & $\mathrm{A} ; \mathrm{B} ; \mathrm{D} ; \mathrm{E} ; \mathrm{F}$ & $\mathrm{C}$ & $\mathrm{C}$ & $\mathrm{C}$ \\
\hline$\alpha$-Pinene & 54.12 & $\leq 1.00 \mathrm{E}-07$ & $\mathrm{C} ; \mathrm{E} ; \mathrm{F}$ & $C ; E ; F$ & $\mathrm{~A} ; \mathrm{B} ; \mathrm{D} ; \mathrm{E}$ & $\mathrm{C} ; \mathrm{F}$ & $\mathrm{A} ; \mathrm{B} ; \mathrm{C} ; \mathrm{F}$ & A; B; D; E \\
\hline 4,4-Dimethyl-2-Pentanone & 1684.33 & $\leq 1.00 \mathrm{E}-07$ & $\mathrm{C} ; \mathrm{F}$ & $\mathrm{F}$ & $\mathrm{A} ; \mathrm{E} ; \mathrm{F}$ & $\mathrm{F}$ & $\mathrm{C} ; \mathrm{F}$ & $\mathrm{A} ; \mathrm{B} ; \mathrm{C} ; \mathrm{D} ; \mathrm{E}$ \\
\hline 1-Penten-3-one & 229.25 & $\leq 1.00 \mathrm{E}-07$ & $\mathrm{C} ; \mathrm{D} ; \mathrm{F}$ & $\mathrm{C} ; \mathrm{D} ; \mathrm{F}$ & $\mathrm{A} ; \mathrm{B} ; \mathrm{D} ; \mathrm{E} ; \mathrm{F}$ & $\mathrm{A} ; \mathrm{B} ; \mathrm{C} ; \mathrm{F}$ & $\mathrm{C} ; \mathrm{F}$ & $\mathrm{A} ; \mathrm{B} ; \mathrm{C} ; \mathrm{D} ; \mathrm{E}$ \\
\hline 5-Butyl-Nonane & 195.21 & $\leq 1.00 \mathrm{E}-07$ & $\mathrm{~B} ; \mathrm{C} ; \mathrm{D} ; \mathrm{E} ; \mathrm{F}$ & $\mathrm{A} ; \mathrm{C} ; \mathrm{D} ; \mathrm{F}$ & $\mathrm{A} ; \mathrm{B} ; \mathrm{D} ; \mathrm{E} ; \mathrm{F}$ & $\mathrm{A} ; \mathrm{B} ; \mathrm{C} ; \mathrm{E}$ & $\mathrm{A} ; \mathrm{C} ; \mathrm{D} ; \mathrm{F}$ & $\mathrm{A} ; \mathrm{B} ; \mathrm{C} ; \mathrm{E}$ \\
\hline 3,6-Dimethyl-Decane & 121.28 & $\leq 1.00 \mathrm{E}-07$ & $\mathrm{~B} ; \mathrm{C} ; \mathrm{D} ; \mathrm{F}$ & $\mathrm{A} ; \mathrm{C} ; \mathrm{D} ; \mathrm{E} ; \mathrm{F}$ & $\mathrm{A} ; \mathrm{B} ; \mathrm{D} ; \mathrm{E} ; \mathrm{F}$ & $\mathrm{A} ; \mathrm{B} ; \mathrm{C} ; \mathrm{E}$ & $\mathrm{A} ; \mathrm{B} ; \mathrm{C} ; \mathrm{D} ; \mathrm{F}$ & $\mathrm{A} ; \mathrm{B} ; \mathrm{C} ; \mathrm{E}$ \\
\hline 2-Methyl-3-Buten-2-ol & 69.73 & $\leq 1.00 \mathrm{E}-07$ & $\mathrm{C}$ & $\mathrm{C}$ & $\mathrm{A} ; \mathrm{B} ; \mathrm{D} ; \mathrm{E} ; \mathrm{F}$ & $\mathrm{C}$ & $\mathrm{C}$ & $\mathrm{C}$ \\
\hline 5-Methyl-Decane & 18.07 & $\leq 1.00 \mathrm{E}-07$ & $\mathrm{~B} ; \mathrm{D} ; \mathrm{F}$ & $\mathrm{A} ; \mathrm{F}$ & $\mathrm{F}$ & $\mathrm{A} ; \mathrm{F}$ & $\mathrm{F}$ & $\mathrm{A} ; \mathrm{B} ; \mathrm{C} ; \mathrm{D} ; \mathrm{E}$ \\
\hline 4-Methyl-Decane & 19.34 & $\leq 1.00 \mathrm{E}-07$ & $\mathrm{~B} ; \mathrm{D} ; \mathrm{E} ; \mathrm{F}$ & $\mathrm{A} ; \mathrm{C}$ & $\mathrm{B} ; \mathrm{D} ; \mathrm{E} ; \mathrm{F}$ & $\mathrm{A} ; \mathrm{C} ; \mathrm{F}$ & $\mathrm{A} ; \mathrm{C}$ & $\mathrm{A} ; \mathrm{C} ; \mathrm{D}$ \\
\hline 2,3-Pentanedione & 66.15 & $\leq 1.00 \mathrm{E}-07$ & $\mathrm{~B} ; \mathrm{C} ; \mathrm{D} ; \mathrm{E} ; \mathrm{F}$ & $\mathrm{A} ; \mathrm{D}$ & $\mathrm{A} ; \mathrm{D}$ & $\mathrm{A} ; \mathrm{B} ; \mathrm{C} ; \mathrm{E} ; \mathrm{F}$ & $\mathrm{A} ; \mathrm{D}$ & $\mathrm{A} ; \mathrm{D}$ \\
\hline Methyl 3,3-Dimethylbutanoate & 38.31 & $\leq 1.00 \mathrm{E}-07$ & $\mathrm{C}$ & $\mathrm{C}$ & $\mathrm{A} ; \mathrm{B} ; \mathrm{D} ; \mathrm{E} ; \mathrm{F}$ & $\mathrm{C}$ & $\mathrm{C}$ & $\mathrm{C}$ \\
\hline 2-Hexanone & 19.88 & $\leq 1.00 \mathrm{E}-07$ & $\mathrm{C}$ & $\mathrm{C}$ & $\mathrm{A} ; \mathrm{B} ; \mathrm{D} ; \mathrm{E} ; \mathrm{F}$ & $\mathrm{C}$ & $\mathrm{C}$ & $\mathrm{C}$ \\
\hline 4-Methyl-Undecane & 98.06 & $\leq 1.00 \mathrm{E}-07$ & $\mathrm{C} ; \mathrm{D} ; \mathrm{E}$ & $\mathrm{C} ; \mathrm{D} ; \mathrm{E}$ & $\mathrm{A} ; \mathrm{B} ; \mathrm{D} ; \mathrm{E} ; \mathrm{F}$ & $\mathrm{A} ; \mathrm{B} ; \mathrm{C} ; \mathrm{E} ; \mathrm{F}$ & $\mathrm{A} ; \mathrm{B} ; \mathrm{C} ; \mathrm{D} ; \mathrm{F}$ & $\mathrm{C} ; \mathrm{D} ; \mathrm{E}$ \\
\hline Hexanal & 133.72 & $\leq 1.00 \mathrm{E}-07$ & $\mathrm{C} ; \mathrm{D} ; \mathrm{F}$ & $\mathrm{C} ; \mathrm{D} ; \mathrm{E} ; \mathrm{F}$ & $\mathrm{A} ; \mathrm{B} ; \mathrm{D} ; \mathrm{E} ; \mathrm{F}$ & $\mathrm{A} ; \mathrm{B} ; \mathrm{C} ; \mathrm{E} ; \mathrm{F}$ & $\mathrm{B} ; \mathrm{C} ; \mathrm{D} ; \mathrm{F}$ & $\mathrm{A} ; \mathrm{B} ; \mathrm{C} ; \mathrm{D} ; \mathrm{E}$ \\
\hline 2-Methyl-1-Propanol & 190.36 & $\leq 1.00 \mathrm{E}-07$ & $\mathrm{~B} ; \mathrm{C} ; \mathrm{D} ; \mathrm{E} ; \mathrm{F}$ & $\mathrm{A} ; \mathrm{D} ; \mathrm{E} ; \mathrm{F}$ & $\mathrm{A} ; \mathrm{E} ; \mathrm{F}$ & $\mathrm{A} ; \mathrm{B} ; \mathrm{E} ; \mathrm{F}$ & $\mathrm{A} ; \mathrm{B} ; \mathrm{C} ; \mathrm{D} ; \mathrm{F}$ & $\mathrm{A} ; \mathrm{B} ; \mathrm{C} ; \mathrm{D} ; \mathrm{E}$ \\
\hline
\end{tabular}




\begin{tabular}{|c|c|c|c|c|c|c|c|c|}
\hline 2-Methyl-2-Pentanol & 162.43 & $\leq 1.00 \mathrm{E}-07$ & $\mathrm{~B} ; \mathrm{D} ; \mathrm{E} ; \mathrm{F}$ & $\mathrm{A} ; \mathrm{C} ; \mathrm{F}$ & $\mathrm{B} ; \mathrm{D} ; \mathrm{E} ; \mathrm{F}$ & $\mathrm{A} ; \mathrm{C} ; \mathrm{F}$ & $\mathrm{A} ; \mathrm{C} ; \mathrm{F}$ & $\mathrm{A} ; \mathrm{B} ; \mathrm{C} ; \mathrm{D} ; \mathrm{E}$ \\
\hline Ethyl-Benzene & 28.80 & $\leq 1.00 \mathrm{E}-07$ & $\mathrm{C}$ & $\mathrm{C} ; \mathrm{F}$ & $\mathrm{A} ; \mathrm{B} ; \mathrm{D} ; \mathrm{E} ; \mathrm{F}$ & $\mathrm{C}$ & $\mathrm{C}$ & $\mathrm{B} ; \mathrm{C}$ \\
\hline 3-Methylbutyl Acetate & 98.57 & $\leq 1.00 \mathrm{E}-07$ & $\mathrm{~B} ; \mathrm{C} ; \mathrm{E}$ & $\mathrm{A} ; \mathrm{D} ; \mathrm{E} ; \mathrm{F}$ & $\mathrm{A} ; \mathrm{D} ; \mathrm{E} ; \mathrm{F}$ & $\mathrm{B} ; \mathrm{C} ; \mathrm{E}$ & $\mathrm{A} ; \mathrm{B} ; \mathrm{C} ; \mathrm{D} ; \mathrm{F}$ & $\mathrm{B} ; \mathrm{C} ; \mathrm{E}$ \\
\hline o-Xylene & 95.67 & $\leq 1.00 \mathrm{E}-07$ & $\mathrm{C} ; \mathrm{F}$ & $\mathrm{C} ; \mathrm{F}$ & $\mathrm{A} ; \mathrm{B} ; \mathrm{D} ; \mathrm{E} ; \mathrm{F}$ & $\mathrm{C} ; \mathrm{F}$ & $\mathrm{C} ; \mathrm{F}$ & $\mathrm{A} ; \mathrm{B} ; \mathrm{C} ; \mathrm{D} ; \mathrm{E}$ \\
\hline 2-Pentanol & 209.29 & $\leq 1.00 \mathrm{E}-07$ & $\mathrm{C}$ & $\mathrm{C}$ & $\mathrm{A} ; \mathrm{B} ; \mathrm{D} ; \mathrm{E} ; \mathrm{F}$ & $\mathrm{C}$ & $\mathrm{C}$ & $\mathrm{C}$ \\
\hline p-Xylene & 56.17 & $\leq 1.00 \mathrm{E}-07$ & $\mathrm{~B} ; \mathrm{C} ; \mathrm{D} ; \mathrm{E} ; \mathrm{F}$ & $\mathrm{A} ; \mathrm{C} ; \mathrm{D} ; \mathrm{E} ; \mathrm{F}$ & $\mathrm{A} ; \mathrm{B} ; \mathrm{E} ; \mathrm{F}$ & $\mathrm{A} ; \mathrm{B} ; \mathrm{E}$ & $\mathrm{A} ; \mathrm{B} ; \mathrm{C} ; \mathrm{D}$ & $\mathrm{A} ; \mathrm{B} ; \mathrm{C}$ \\
\hline 5-Methyl-2-Hexanone & 274.44 & $\leq 1.00 \mathrm{E}-07$ & $\mathrm{~B} ; \mathrm{C} ; \mathrm{D} ; \mathrm{F}$ & $\mathrm{A} ; \mathrm{C} ; \mathrm{F}$ & $\mathrm{A} ; \mathrm{B} ; \mathrm{D} ; \mathrm{E} ; \mathrm{F}$ & $\mathrm{A} ; \mathrm{C} ; \mathrm{F}$ & $\mathrm{C} ; \mathrm{F}$ & $\mathrm{A} ; \mathrm{B} ; \mathrm{C} ; \mathrm{D} ; \mathrm{E}$ \\
\hline Methyl 2-Ethylpentanoate & 78.94 & $\leq 1.00 \mathrm{E}-07$ & $\mathrm{C}$ & $\mathrm{C}$ & $\mathrm{A} ; \mathrm{B} ; \mathrm{D} ; \mathrm{E} ; \mathrm{F}$ & $\mathrm{C}$ & $\mathrm{C}$ & $\mathrm{C}$ \\
\hline 1-Butanol & 72.01 & $\leq 1.00 \mathrm{E}-07$ & $\mathrm{C} ; \mathrm{E}$ & $\mathrm{C} ; \mathrm{E}$ & $\mathrm{A} ; \mathrm{B} ; \mathrm{D} ; \mathrm{E}$ & $\mathrm{C} ; \mathrm{E}$ & $\mathrm{A} ; \mathrm{B} ; \mathrm{D} ; \mathrm{F}$ & $\mathrm{C} ; \mathrm{E}$ \\
\hline$\alpha$-Phellandrene & 43.64 & $\leq 1.00 \mathrm{E}-07$ & $\mathrm{~F}$ & $\mathrm{~F}$ & $\mathrm{~F}$ & $\mathrm{~F}$ & $\mathrm{~F}$ & $\mathrm{~A} ; \mathrm{B} ; \mathrm{C} ; \mathrm{D} ; \mathrm{E}$ \\
\hline 2-Methyl-3-Pentanol & 214.21 & $\leq 1.00 \mathrm{E}-07$ & $\mathrm{C} ; \mathrm{D}$ & C; D & $\mathrm{A} ; \mathrm{B} ; \mathrm{D} ; \mathrm{E} ; \mathrm{F}$ & $\mathrm{A} ; \mathrm{B} ; \mathrm{C} ; \mathrm{E} ; \mathrm{F}$ & $\mathrm{C} ; \mathrm{D}$ & $\mathrm{C} ; \mathrm{D}$ \\
\hline 1-Penten-3-ol & 26.15 & $\leq 1.00 \mathrm{E}-07$ & $\mathrm{~B} ; \mathrm{C} ; \mathrm{F}$ & $\mathrm{A} ; \mathrm{C} ; \mathrm{D}$ & $\mathrm{A} ; \mathrm{B} ; \mathrm{D} ; \mathrm{E}$ & $\mathrm{B} ; \mathrm{C} ; \mathrm{E} ; \mathrm{F}$ & $\mathrm{C} ; \mathrm{D}$ & $\mathrm{A} ; \mathrm{D}$ \\
\hline$\alpha$-Terpinene & 162.78 & $\leq 1.00 \mathrm{E}-07$ & $\mathrm{D} ; \mathrm{F}$ & $\mathrm{D} ; \mathrm{F}$ & $\mathrm{D} ; \mathrm{F}$ & $\mathrm{A} ; \mathrm{B} ; \mathrm{C} ; \mathrm{E} ; \mathrm{F}$ & $\mathrm{D} ; \mathrm{F}$ & $\mathrm{A} ; \mathrm{B} ; \mathrm{C} ; \mathrm{D} ; \mathrm{E}$ \\
\hline Butyl Prop-2-enoate & 17.37 & $\leq 1.00 \mathrm{E}-07$ & $\mathrm{C} ; \mathrm{F}$ & $\mathrm{C} ; \mathrm{F}$ & $\mathrm{A} ; \mathrm{B} ; \mathrm{D} ; \mathrm{E}$ & $\mathrm{C} ; \mathrm{F}$ & $\mathrm{C} ; \mathrm{F}$ & $\mathrm{A} ; \mathrm{B} ; \mathrm{D} ; \mathrm{E}$ \\
\hline m-Xylene & 141.47 & $\leq 1.00 \mathrm{E}-07$ & $\mathrm{~B} ; \mathrm{C} ; \mathrm{E} ; \mathrm{F}$ & $\mathrm{A} ; \mathrm{C} ; \mathrm{D} ; \mathrm{F}$ & $\mathrm{A} ; \mathrm{B} ; \mathrm{D} ; \mathrm{E} ; \mathrm{F}$ & $\mathrm{B} ; \mathrm{C} ; \mathrm{E} ; \mathrm{F}$ & $\mathrm{A} ; \mathrm{C} ; \mathrm{D} ; \mathrm{F}$ & $\mathrm{A} ; \mathrm{B} ; \mathrm{C} ; \mathrm{D} ; \mathrm{E}$ \\
\hline 2-Heptanone & 266.55 & $\leq 1.00 \mathrm{E}-07$ & $\mathrm{~B} ; \mathrm{E} ; \mathrm{F}$ & $\mathrm{A} ; \mathrm{C} ; \mathrm{D} ; \mathrm{E} ; \mathrm{F}$ & $\mathrm{B} ; \mathrm{D} ; \mathrm{E} ; \mathrm{F}$ & $\mathrm{B} ; \mathrm{C} ; \mathrm{E} ; \mathrm{F}$ & $\mathrm{A} ; \mathrm{B} ; \mathrm{C} ; \mathrm{D} ; \mathrm{F}$ & $\mathrm{A} ; \mathrm{B} ; \mathrm{C} ; \mathrm{D} ; \mathrm{E}$ \\
\hline Heptanal & 130.47 & $\leq 1.00 \mathrm{E}-07$ & $\mathrm{~B} ; \mathrm{C} ; \mathrm{F}$ & $\mathrm{A} ; \mathrm{C} ; \mathrm{D} ; \mathrm{E} ; \mathrm{F}$ & $\mathrm{A} ; \mathrm{B} ; \mathrm{D} ; \mathrm{E} ; \mathrm{F}$ & $\mathrm{B} ; \mathrm{C} ; \mathrm{E} ; \mathrm{F}$ & $\mathrm{B} ; \mathrm{C} ; \mathrm{F}$ & $\mathrm{A} ; \mathrm{B} ; \mathrm{C} ; \mathrm{D} ; \mathrm{E}$ \\
\hline D-Limonene & 241.25 & $\leq 1.00 \mathrm{E}-07$ & $\mathrm{C} ; \mathrm{E} ; \mathrm{F}$ & $\mathrm{C} ; \mathrm{E} ; \mathrm{F}$ & $\mathrm{A} ; \mathrm{B} ; \mathrm{D} ; \mathrm{E} ; \mathrm{F}$ & $\mathrm{C} ; \mathrm{E} ; \mathrm{F}$ & $\mathrm{A} ; \mathrm{B} ; \mathrm{C} ; \mathrm{D}$ & $\mathrm{A} ; \mathrm{B} ; \mathrm{C} ; \mathrm{D}$ \\
\hline 3-Hexanol & 248.12 & $\leq 1.00 \mathrm{E}-07$ & $\mathrm{~B} ; \mathrm{D} ; \mathrm{E} ; \mathrm{F}$ & $\mathrm{A} ; \mathrm{C} ; \mathrm{D} ; \mathrm{F}$ & $\mathrm{B} ; \mathrm{D} ; \mathrm{E}$ & $\mathrm{A} ; \mathrm{B} ; \mathrm{C} ; \mathrm{E} ; \mathrm{F}$ & $\mathrm{A} ; \mathrm{C} ; \mathrm{D} ; \mathrm{F}$ & $\mathrm{A} ; \mathrm{B} ; \mathrm{D} ; \mathrm{E}$ \\
\hline$\beta$-Phellandrene & 22.59 & $\leq 1.00 \mathrm{E}-07$ & $\mathrm{~F}$ & $\mathrm{~F}$ & $\mathrm{~F}$ & $\mathrm{~F}$ & $\mathrm{~F}$ & $\mathrm{~A} ; \mathrm{B} ; \mathrm{C} ; \mathrm{D} ; \mathrm{E}$ \\
\hline Eucalyptol & 58.70 & $\leq 1.00 \mathrm{E}-07$ & $\mathrm{D} ; \mathrm{F}$ & $\mathrm{D} ; \mathrm{F}$ & $\mathrm{D} ; \mathrm{F}$ & $\mathrm{A} ; \mathrm{B} ; \mathrm{C} ; \mathrm{E} ; \mathrm{F}$ & $\mathrm{D} ; \mathrm{F}$ & $\mathrm{A} ; \mathrm{B} ; \mathrm{C} ; \mathrm{D} ; \mathrm{E}$ \\
\hline m-Menthane & 76.50 & $\leq 1.00 \mathrm{E}-07$ & $\mathrm{C} ; \mathrm{E} ; \mathrm{F}$ & $\mathrm{C} ; \mathrm{F}$ & $\mathrm{A} ; \mathrm{B} ; \mathrm{D} ; \mathrm{E}$ & $\mathrm{C} ; \mathrm{F}$ & $\mathrm{A} ; \mathrm{C} ; \mathrm{F}$ & $\mathrm{A} ; \mathrm{B} ; \mathrm{D} ; \mathrm{E}$ \\
\hline 4-Methyl-2-Heptanone & 1066.87 & $\leq 1.00 \mathrm{E}-07$ & $\mathrm{~B} ; \mathrm{F}$ & $\mathrm{A} ; \mathrm{C} ; \mathrm{D} ; \mathrm{E} ; \mathrm{F}$ & $\mathrm{B} ; \mathrm{F}$ & $\mathrm{B} ; \mathrm{F}$ & $\mathrm{B} ; \mathrm{F}$ & $\mathrm{A} ; \mathrm{B} ; \mathrm{C} ; \mathrm{D} ; \mathrm{E}$ \\
\hline 3-Methyl-2-Pentanol & 504.97 & $\leq 1.00 \mathrm{E}-07$ & $\mathrm{~B} ; \mathrm{C} ; \mathrm{D} ; \mathrm{E} ; \mathrm{F}$ & $\mathrm{A} ; \mathrm{D} ; \mathrm{F}$ & $\mathrm{A} ; \mathrm{D} ; \mathrm{F}$ & $\mathrm{A} ; \mathrm{B} ; \mathrm{C} ; \mathrm{E}$ & A; D; F & $\mathrm{A} ; \mathrm{B} ; \mathrm{C} ; \mathrm{E}$ \\
\hline 2-Methyl-1-Butanol & 26.64 & $\leq 1.00 \mathrm{E}-07$ & $\mathrm{D} ; \mathrm{F}$ & $\mathrm{D} ; \mathrm{F}$ & $\mathrm{D} ; \mathrm{F}$ & $\mathrm{A} ; \mathrm{B} ; \mathrm{C} ; \mathrm{E} ; \mathrm{F}$ & $\mathrm{D} ; \mathrm{F}$ & $\mathrm{A} ; \mathrm{B} ; \mathrm{C} ; \mathrm{D} ; \mathrm{E}$ \\
\hline 3-Methyl-1-Butanol & 23.35 & $\leq 1.00 \mathrm{E}-07$ & $\mathrm{~B} ; \mathrm{D} ; \mathrm{F}$ & $\mathrm{A} ; \mathrm{F}$ & $\mathrm{F}$ & $\mathrm{A} ; \mathrm{F}$ & $\mathrm{F}$ & $\mathrm{A} ; \mathrm{B} ; \mathrm{C} ; \mathrm{D} ; \mathrm{E}$ \\
\hline o-Ethyltoluene & 69.98 & $\leq 1.00 \mathrm{E}-07$ & $\mathrm{~B} ; \mathrm{C} ; \mathrm{D} ; \mathrm{E} ; \mathrm{F}$ & $\mathrm{A} ; \mathrm{C} ; \mathrm{E} ; \mathrm{F}$ & $\mathrm{A} ; \mathrm{B} ; \mathrm{D} ; \mathrm{E} ; \mathrm{F}$ & $\mathrm{A} ; \mathrm{C} ; \mathrm{E}$ & $\mathrm{A} ; \mathrm{B} ; \mathrm{C} ; \mathrm{D} ; \mathrm{F}$ & $\mathrm{A} ; \mathrm{B} ; \mathrm{C} ; \mathrm{E}$ \\
\hline m-Ethyltoluene & 157.66 & $\leq 1.00 \mathrm{E}-07$ & $\mathrm{~B} ; \mathrm{C} ; \mathrm{D} ; \mathrm{E}$ & $\mathrm{A} ; \mathrm{C} ; \mathrm{D} ; \mathrm{E}$ & $\mathrm{A} ; \mathrm{B} ; \mathrm{D} ; \mathrm{E} ; \mathrm{F}$ & $\mathrm{A} ; \mathrm{B} ; \mathrm{C} ; \mathrm{E} ; \mathrm{F}$ & $\mathrm{A} ; \mathrm{B} ; \mathrm{C} ; \mathrm{D} ; \mathrm{F}$ & $\mathrm{C} ; \mathrm{D} ; \mathrm{E}$ \\
\hline 2-Hexanol & 171.88 & $\leq 1.00 \mathrm{E}-07$ & $\mathrm{~B} ; \mathrm{C} ; \mathrm{D} ; \mathrm{E} ; \mathrm{F}$ & $\mathrm{A} ; \mathrm{C} ; \mathrm{D} ; \mathrm{E} ; \mathrm{F}$ & $\mathrm{A} ; \mathrm{B}$ & $\mathrm{A} ; \mathrm{B}$ & $\mathrm{A} ; \mathrm{B}$ & $\mathrm{A} ; \mathrm{B}$ \\
\hline Ethylene Glycol Ethyl Ether & 32.70 & $\leq 1.00 \mathrm{E}-07$ & $\mathrm{D}$ & $\mathrm{D}$ & $\mathrm{D}$ & $\mathrm{A} ; \mathrm{B} ; \mathrm{C} ; \mathrm{E} ; \mathrm{F}$ & $\mathrm{D}$ & $\mathrm{D}$ \\
\hline
\end{tabular}




\begin{tabular}{|c|c|c|c|c|c|c|c|c|}
\hline 6-Methyl-2-Heptanone & 106.44 & $\leq 1.00 \mathrm{E}-07$ & $\mathrm{~F}$ & $\mathrm{C} ; \mathrm{F}$ & $\mathrm{B} ; \mathrm{D} ; \mathrm{E} ; \mathrm{F}$ & $\mathrm{C} ; \mathrm{F}$ & $\mathrm{C} ; \mathrm{F}$ & $\mathrm{A} ; \mathrm{B} ; \mathrm{C} ; \mathrm{D} ; \mathrm{E}$ \\
\hline Pseudocumene & 38.50 & $\leq 1.00 \mathrm{E}-07$ & $\mathrm{~B} ; \mathrm{C} ; \mathrm{D} ; \mathrm{F}$ & $\mathrm{A} ; \mathrm{C} ; \mathrm{E} ; \mathrm{F}$ & A; B; D; E; F & $\mathrm{A} ; \mathrm{C} ; \mathrm{E}$ & $\mathrm{B} ; \mathrm{C} ; \mathrm{D} ; \mathrm{F}$ & $\mathrm{A} ; \mathrm{C} ; \mathrm{E}$ \\
\hline 4,6-Dimethyl-2-Heptanone & 179.79 & $\leq 1.00 \mathrm{E}-07$ & $\mathrm{~F}$ & $\mathrm{~F}$ & $\mathrm{~F}$ & $\mathrm{~F}$ & $\mathrm{~F}$ & $\mathrm{~A} ; \mathrm{B} ; \mathrm{C} ; \mathrm{D} ; \mathrm{E}$ \\
\hline 2,3-Dimethyl-2-Hexanol & 1243.14 & $\leq 1.00 \mathrm{E}-07$ & $\mathrm{C} ; \mathrm{F}$ & $\mathrm{C} ; \mathrm{F}$ & $\mathrm{A} ; \mathrm{B} ; \mathrm{D} ; \mathrm{E} ; \mathrm{F}$ & $\mathrm{C} ; \mathrm{F}$ & $\mathrm{C} ; \mathrm{F}$ & $\mathrm{A} ; \mathrm{B} ; \mathrm{C} ; \mathrm{D} ; \mathrm{E}$ \\
\hline 3-Octanone & 137.45 & $\leq 1.00 \mathrm{E}-07$ & $\mathrm{~B} ; \mathrm{C} ; \mathrm{D} ; \mathrm{E} ; \mathrm{F}$ & $\mathrm{A} ; \mathrm{D} ; \mathrm{F}$ & $\mathrm{A} ; \mathrm{D} ; \mathrm{F}$ & $\mathrm{A} ; \mathrm{B} ; \mathrm{C} ; \mathrm{E}$ & $\mathrm{A} ; \mathrm{D} ; \mathrm{F}$ & $\mathrm{A} ; \mathrm{B} ; \mathrm{C} ; \mathrm{E}$ \\
\hline Styrene & 34.57 & $\leq 1.00 \mathrm{E}-07$ & C; D & $\mathrm{C} ; \mathrm{D} ; \mathrm{E}$ & $\mathrm{A} ; \mathrm{B} ; \mathrm{D} ; \mathrm{E} ; \mathrm{F}$ & $\mathrm{A} ; \mathrm{B} ; \mathrm{C} ; \mathrm{F}$ & $\mathrm{B} ; \mathrm{C} ; \mathrm{F}$ & $\mathrm{C} ; \mathrm{D} ; \mathrm{E}$ \\
\hline 5-Methyl-2-Heptanone & 33.99 & $\leq 1.00 \mathrm{E}-07$ & $\mathrm{~B} ; \mathrm{F}$ & $\mathrm{A} ; \mathrm{C} ; \mathrm{E} ; \mathrm{F}$ & $\mathrm{B} ; \mathrm{F}$ & $\mathrm{F}$ & $\mathrm{B} ; \mathrm{F}$ & $\mathrm{A} ; \mathrm{B} ; \mathrm{C} ; \mathrm{D} ; \mathrm{E}$ \\
\hline 1-Pentanol & 49.63 & $\leq 1.00 \mathrm{E}-07$ & $C ; E ; F$ & $C ; E ; F$ & $\mathrm{~A} ; \mathrm{B} ; \mathrm{D}$ & $C ; E ; F$ & $\mathrm{~A} ; \mathrm{B} ; \mathrm{D}$ & $\mathrm{A} ; \mathrm{B} ; \mathrm{D}$ \\
\hline p-Ethyltoluene & 92.19 & $\leq 1.00 \mathrm{E}-07$ & $\mathrm{~B} ; \mathrm{C} ; \mathrm{D} ; \mathrm{E} ; \mathrm{F}$ & $\mathrm{A} ; \mathrm{C} ; \mathrm{D} ; \mathrm{E}$ & A; B; D; E; F & $\mathrm{A} ; \mathrm{B} ; \mathrm{C} ; \mathrm{F}$ & $\mathrm{A} ; \mathrm{B} ; \mathrm{C} ; \mathrm{F}$ & $\mathrm{A} ; \mathrm{C} ; \mathrm{D} ; \mathrm{E}$ \\
\hline p-Cymene & 94.51 & $\leq 1.00 \mathrm{E}-07$ & $\mathrm{D} ; \mathrm{F}$ & $\mathrm{D} ; \mathrm{F}$ & $\mathrm{D} ; \mathrm{F}$ & $\mathrm{A} ; \mathrm{B} ; \mathrm{C} ; \mathrm{E} ; \mathrm{F}$ & $\mathrm{D} ; \mathrm{F}$ & $\mathrm{A} ; \mathrm{B} ; \mathrm{C} ; \mathrm{D} ; \mathrm{E}$ \\
\hline Mesitylene & 112.91 & $\leq 1.00 \mathrm{E}-07$ & $\mathrm{~B} ; \mathrm{C} ; \mathrm{E}$ & $\mathrm{A} ; \mathrm{D} ; \mathrm{E} ; \mathrm{F}$ & $\mathrm{A} ; \mathrm{D} ; \mathrm{E} ; \mathrm{F}$ & $\mathrm{B} ; \mathrm{C} ; \mathrm{E}$ & $\mathrm{A} ; \mathrm{B} ; \mathrm{C} ; \mathrm{D} ; \mathrm{F}$ & $\mathrm{B} ; \mathrm{C} ; \mathrm{E}$ \\
\hline 4-Methyl-4-Heptanol & 41.36 & $\leq 1.00 \mathrm{E}-07$ & $\mathrm{~B} ; \mathrm{C} ; \mathrm{D} ; \mathrm{E} ; \mathrm{F}$ & $\mathrm{A} ; \mathrm{C} ; \mathrm{F}$ & $\mathrm{A} ; \mathrm{B} ; \mathrm{F}$ & $\mathrm{A} ; \mathrm{F}$ & $\mathrm{A} ; \mathrm{F}$ & $\mathrm{A} ; \mathrm{B} ; \mathrm{C} ; \mathrm{D} ; \mathrm{E}$ \\
\hline 4-Heptanol & 50.36 & $\leq 1.00 \mathrm{E}-07$ & $\mathrm{D} ; \mathrm{F}$ & $\mathrm{F}$ & $\mathrm{F}$ & $\mathrm{A} ; \mathrm{F}$ & $\mathrm{F}$ & $\mathrm{A} ; \mathrm{B} ; \mathrm{C} ; \mathrm{D} ; \mathrm{E}$ \\
\hline 2-Octanone & 130.90 & $\leq 1.00 \mathrm{E}-07$ & $\mathrm{~B} ; \mathrm{E} ; \mathrm{F}$ & $\mathrm{A} ; \mathrm{C} ; \mathrm{D} ; \mathrm{E} ; \mathrm{F}$ & $\mathrm{B} ; \mathrm{D} ; \mathrm{E} ; \mathrm{F}$ & $\mathrm{B} ; \mathrm{C} ; \mathrm{E} ; \mathrm{F}$ & $\mathrm{A} ; \mathrm{B} ; \mathrm{C} ; \mathrm{D}$ & $\mathrm{A} ; \mathrm{B} ; \mathrm{C} ; \mathrm{D}$ \\
\hline Octanal & 289.51 & $\leq 1.00 \mathrm{E}-07$ & $\mathrm{C} ; \mathrm{D} ; \mathrm{E} ; \mathrm{F}$ & C; D; E; F & $\mathrm{A} ; \mathrm{B} ; \mathrm{D} ; \mathrm{E}$ & $\mathrm{A} ; \mathrm{B} ; \mathrm{C} ; \mathrm{F}$ & $\mathrm{A} ; \mathrm{B} ; \mathrm{C} ; \mathrm{F}$ & $\mathrm{A} ; \mathrm{B} ; \mathrm{D} ; \mathrm{E}$ \\
\hline 3-Hydroxy-2-Butanone & 34.07 & $\leq 1.00 \mathrm{E}-07$ & $\mathrm{~B} ; \mathrm{C} ; \mathrm{F}$ & $\mathrm{A} ; \mathrm{C} ; \mathrm{D} ; \mathrm{E} ; \mathrm{F}$ & $\mathrm{A} ; \mathrm{B} ; \mathrm{D} ; \mathrm{E}$ & $\mathrm{B} ; \mathrm{C} ; \mathrm{F}$ & $\mathrm{B} ; \mathrm{C} ; \mathrm{F}$ & $\mathrm{A} ; \mathrm{B} ; \mathrm{D} ; \mathrm{E}$ \\
\hline 1-Octen-3-one & 69.53 & $\leq 1.00 \mathrm{E}-07$ & $\mathrm{~B} ; \mathrm{C} ; \mathrm{D} ; \mathrm{E}$ & $\mathrm{A} ; \mathrm{E} ; \mathrm{F}$ & $\mathrm{A} ; \mathrm{F}$ & $\mathrm{A} ; \mathrm{F}$ & $\mathrm{A} ; \mathrm{B} ; \mathrm{F}$ & $\mathrm{B} ; \mathrm{C} ; \mathrm{D} ; \mathrm{E}$ \\
\hline trans-2-Pentenol & 15.06 & $\leq 1.00 \mathrm{E}-07$ & $\mathrm{~B} ; \mathrm{C} ; \mathrm{D} ; \mathrm{E}$ & $\mathrm{A} ; \mathrm{F}$ & $\mathrm{A} ; \mathrm{F}$ & A & A & $\mathrm{B} ; \mathrm{C}$ \\
\hline 2,2,6-Trimethyl-Cyclohexanone & 83.07 & $\leq 1.00 \mathrm{E}-07$ & $\mathrm{~B} ; \mathrm{C} ; \mathrm{E} ; \mathrm{F}$ & $\mathrm{A} ; \mathrm{C} ; \mathrm{D} ; \mathrm{F}$ & A; B; D; E; F & $\mathrm{B} ; \mathrm{C} ; \mathrm{E} ; \mathrm{F}$ & $\mathrm{A} ; \mathrm{C} ; \mathrm{D} ; \mathrm{F}$ & $\mathrm{A} ; \mathrm{B} ; \mathrm{C} ; \mathrm{D} ; \mathrm{E}$ \\
\hline 2-Heptanol & 67.77 & $\leq 1.00 \mathrm{E}-07$ & $\mathrm{C} ; \mathrm{E} ; \mathrm{F}$ & $C ; E ; F$ & $\mathrm{~A} ; \mathrm{B} ; \mathrm{D} ; \mathrm{E} ; \mathrm{F}$ & $\mathrm{C} ; \mathrm{F}$ & $\mathrm{A} ; \mathrm{B} ; \mathrm{C} ; \mathrm{F}$ & $\mathrm{A} ; \mathrm{B} ; \mathrm{C} ; \mathrm{D} ; \mathrm{E}$ \\
\hline cis-2-Pentenol & 20.15 & $\leq 1.00 \mathrm{E}-07$ & $\mathrm{~B} ; \mathrm{C} ; \mathrm{E} ; \mathrm{F}$ & $\mathrm{A} ; \mathrm{D}$ & $\mathrm{A} ; \mathrm{D}$ & $\mathrm{B} ; \mathrm{C} ; \mathrm{E} ; \mathrm{F}$ & $\mathrm{A} ; \mathrm{D}$ & $\mathrm{A} ; \mathrm{D}$ \\
\hline 1-Hydroxy-2-Propanone & 192.90 & $\leq 1.00 \mathrm{E}-07$ & $C ; D ; E ; F$ & $\mathrm{D}$ & $\mathrm{A} ; \mathrm{D}$ & $\mathrm{A} ; \mathrm{B} ; \mathrm{C} ; \mathrm{E} ; \mathrm{F}$ & $\mathrm{A} ; \mathrm{D}$ & $\mathrm{A} ; \mathrm{D}$ \\
\hline 5-Methyl-4-Hexen-3-one & 28.36 & $\leq 1.00 \mathrm{E}-07$ & $\mathrm{~B} ; \mathrm{D} ; \mathrm{E}$ & $\mathrm{A} ; \mathrm{C} ; \mathrm{F}$ & $\mathrm{B} ; \mathrm{D} ; \mathrm{E}$ & $\mathrm{A} ; \mathrm{C} ; \mathrm{E} ; \mathrm{F}$ & $\mathrm{A} ; \mathrm{C} ; \mathrm{D} ; \mathrm{F}$ & $\mathrm{B} ; \mathrm{D} ; \mathrm{E}$ \\
\hline Hemimellitene & 56.95 & $\leq 1.00 \mathrm{E}-07$ & $\mathrm{~B} ; \mathrm{C} ; \mathrm{F}$ & $\mathrm{A} ; \mathrm{C} ; \mathrm{D} ; \mathrm{E}$ & $\mathrm{A} ; \mathrm{B} ; \mathrm{D} ; \mathrm{E} ; \mathrm{F}$ & $\mathrm{B} ; \mathrm{C} ; \mathrm{E} ; \mathrm{F}$ & $\mathrm{B} ; \mathrm{C} ; \mathrm{D}$ & $\mathrm{A} ; \mathrm{C} ; \mathrm{D}$ \\
\hline 6-Methyl-5-Hepten-2-one & 98.82 & $\leq 1.00 \mathrm{E}-07$ & $\mathrm{~B} ; \mathrm{E} ; \mathrm{F}$ & $\mathrm{A} ; \mathrm{C} ; \mathrm{F}$ & $\mathrm{B} ; \mathrm{D} ; \mathrm{E} ; \mathrm{F}$ & $\mathrm{C} ; \mathrm{F}$ & $\mathrm{A} ; \mathrm{C} ; \mathrm{F}$ & $\mathrm{A} ; \mathrm{B} ; \mathrm{C} ; \mathrm{D} ; \mathrm{E}$ \\
\hline 2-Pentene & 63.40 & $\leq 1.00 \mathrm{E}-07$ & $\mathrm{~B} ; \mathrm{C} ; \mathrm{D} ; \mathrm{E} ; \mathrm{F}$ & $\mathrm{A} ; \mathrm{C}$ & A; B; D; E; F & $\mathrm{A} ; \mathrm{C} ; \mathrm{E}$ & $\mathrm{A} ; \mathrm{C} ; \mathrm{E} ; \mathrm{F}$ & $\mathrm{A} ; \mathrm{C} ; \mathrm{E}$ \\
\hline 1-Hexanol & 102.15 & $\leq 1.00 \mathrm{E}-07$ & $\mathrm{~B} ; \mathrm{E} ; \mathrm{F}$ & $\mathrm{A} ; \mathrm{C} ; \mathrm{D} ; \mathrm{F}$ & $\mathrm{B} ; \mathrm{E} ; \mathrm{F}$ & $\mathrm{B} ; \mathrm{E} ; \mathrm{F}$ & $\mathrm{A} ; \mathrm{C} ; \mathrm{D} ; \mathrm{F}$ & $\mathrm{A} ; \mathrm{B} ; \mathrm{C} ; \mathrm{D} ; \mathrm{E}$ \\
\hline 2-Cyclopenten-1-one & 52.26 & $\leq 1.00 \mathrm{E}-07$ & $\mathrm{~B} ; \mathrm{C} ; \mathrm{D} ; \mathrm{F}$ & $\mathrm{A} ; \mathrm{D} ; \mathrm{E}$ & $\mathrm{A} ; \mathrm{D} ; \mathrm{E}$ & $\mathrm{A} ; \mathrm{B} ; \mathrm{C} ; \mathrm{E} ; \mathrm{F}$ & $\mathrm{B} ; \mathrm{C} ; \mathrm{D} ; \mathrm{F}$ & $\mathrm{A} ; \mathrm{D} ; \mathrm{E}$ \\
\hline 2-Methyl-2-Cyclopenten-1-one & 7.91 & $7.60 \mathrm{E}-05$ & $\mathrm{~B} ; \mathrm{C} ; \mathrm{D} ; \mathrm{E} ; \mathrm{F}$ & A & A & A & A & A \\
\hline$\beta$-Methy-Styrene & 218.18 & $\leq 1.00 \mathrm{E}-07$ & $\mathrm{~B} ; \mathrm{C} ; \mathrm{D} ; \mathrm{E} ; \mathrm{F}$ & $\mathrm{A} ; \mathrm{C} ; \mathrm{D} ; \mathrm{E} ; \mathrm{F}$ & $\mathrm{A} ; \mathrm{B} ; \mathrm{E} ; \mathrm{F}$ & $\mathrm{A} ; \mathrm{B} ; \mathrm{E} ; \mathrm{F}$ & $\mathrm{A} ; \mathrm{B} ; \mathrm{C} ; \mathrm{D} ; \mathrm{F}$ & $\mathrm{A} ; \mathrm{B} ; \mathrm{C} ; \mathrm{D} ; \mathrm{E}$ \\
\hline
\end{tabular}




\begin{tabular}{|c|c|c|c|c|c|c|c|c|}
\hline 4-Octanol & 52.41 & $\leq 1.00 \mathrm{E}-07$ & $\mathrm{C} ; \mathrm{F}$ & $\mathrm{C} ; \mathrm{F}$ & $\mathrm{A} ; \mathrm{B} ; \mathrm{D} ; \mathrm{E} ; \mathrm{F}$ & $\mathrm{C} ; \mathrm{F}$ & $\mathrm{C} ; \mathrm{F}$ & $\mathrm{A} ; \mathrm{B} ; \mathrm{C} ; \mathrm{D} ; \mathrm{E}$ \\
\hline cis-3-Hexen-1-ol & 62.26 & $\leq 1.00 \mathrm{E}-07$ & $\mathrm{~B} ; \mathrm{C} ; \mathrm{D} ; \mathrm{E} ; \mathrm{F}$ & $\mathrm{A} ; \mathrm{C} ; \mathrm{E} ; \mathrm{F}$ & $\mathrm{A} ; \mathrm{B} ; \mathrm{D} ; \mathrm{E} ; \mathrm{F}$ & $\mathrm{A} ; \mathrm{C} ; \mathrm{F}$ & $\mathrm{A} ; \mathrm{B} ; \mathrm{C} ; \mathrm{F}$ & $\mathrm{A} ; \mathrm{B} ; \mathrm{C} ; \mathrm{D} ; \mathrm{E}$ \\
\hline 2-Nonanone & 129.63 & $\leq 1.00 \mathrm{E}-07$ & $\mathrm{~B} ; \mathrm{E} ; \mathrm{F}$ & $\mathrm{A} ; \mathrm{C} ; \mathrm{D} ; \mathrm{E} ; \mathrm{F}$ & $\mathrm{B} ; \mathrm{E} ; \mathrm{F}$ & $\mathrm{B} ; \mathrm{E}$ & $\mathrm{A} ; \mathrm{B} ; \mathrm{C} ; \mathrm{D} ; \mathrm{F}$ & $\mathrm{A} ; \mathrm{B} ; \mathrm{C} ; \mathrm{E}$ \\
\hline Nonanal & 250.24 & $\leq 1.00 \mathrm{E}-07$ & $\mathrm{C} ; \mathrm{D} ; \mathrm{E} ; \mathrm{F}$ & $\mathrm{C} ; \mathrm{D} ; \mathrm{E} ; \mathrm{F}$ & A; B; D; E; F & $\mathrm{A} ; \mathrm{B} ; \mathrm{C} ; \mathrm{F}$ & $\mathrm{A} ; \mathrm{B} ; \mathrm{C} ; \mathrm{F}$ & $\mathrm{A} ; \mathrm{B} ; \mathrm{C} ; \mathrm{D} ; \mathrm{E}$ \\
\hline Ethylene Glycol Butyl Ether & 349.45 & $\leq 1.00 \mathrm{E}-07$ & $\mathrm{~B} ; \mathrm{C} ; \mathrm{D} ; \mathrm{E} ; \mathrm{F}$ & $\mathrm{A} ; \mathrm{C} ; \mathrm{D} ; \mathrm{E}$ & A; B; D; E; F & $\mathrm{A} ; \mathrm{B} ; \mathrm{C} ; \mathrm{E} ; \mathrm{F}$ & $\mathrm{A} ; \mathrm{B} ; \mathrm{C} ; \mathrm{D} ; \mathrm{F}$ & $\mathrm{A} ; \mathrm{C} ; \mathrm{D} ; \mathrm{E}$ \\
\hline 1-Ethyl-1-Methyl-Cyclopentane & 230.15 & $\leq 1.00 \mathrm{E}-07$ & $\mathrm{C} ; \mathrm{F}$ & $\mathrm{C} ; \mathrm{F}$ & $\mathrm{A} ; \mathrm{B} ; \mathrm{D} ; \mathrm{E} ; \mathrm{F}$ & $\mathrm{C} ; \mathrm{F}$ & $\mathrm{C} ; \mathrm{F}$ & $\mathrm{A} ; \mathrm{B} ; \mathrm{C} ; \mathrm{D} ; \mathrm{E}$ \\
\hline 3-Octen-2-one & 42.77 & $\leq 1.00 \mathrm{E}-07$ & B & $\mathrm{A} ; \mathrm{C} ; \mathrm{D} ; \mathrm{E} ; \mathrm{F}$ & B & B & B & B \\
\hline 2-Octanol & 229.83 & $\leq 1.00 \mathrm{E}-07$ & $\mathrm{~B} ; \mathrm{C} ; \mathrm{E} ; \mathrm{F}$ & $\mathrm{A} ; \mathrm{C} ; \mathrm{D} ; \mathrm{E} ; \mathrm{F}$ & A; B; D; E; F & $\mathrm{B} ; \mathrm{C} ; \mathrm{E} ; \mathrm{F}$ & $\mathrm{A} ; \mathrm{B} ; \mathrm{C} ; \mathrm{D} ; \mathrm{F}$ & $\mathrm{A} ; \mathrm{B} ; \mathrm{C} ; \mathrm{D} ; \mathrm{E}$ \\
\hline 3-Ethyl-2-Methyl-1,3-Hexadiene & 1111.23 & $\leq 1.00 \mathrm{E}-07$ & $\mathrm{C} ; \mathrm{F}$ & $\mathrm{C} ; \mathrm{F}$ & $\mathrm{A} ; \mathrm{B} ; \mathrm{D} ; \mathrm{E} ; \mathrm{F}$ & $\mathrm{C} ; \mathrm{F}$ & $\mathrm{C} ; \mathrm{F}$ & $\mathrm{A} ; \mathrm{B} ; \mathrm{C} ; \mathrm{D} ; \mathrm{E}$ \\
\hline 1,3-Bis(1,1-Dimethylethyl)-Benzene & 249.95 & $\leq 1.00 \mathrm{E}-07$ & $\mathrm{~F}$ & $\mathrm{~F}$ & $\mathrm{~F}$ & $\mathrm{~F}$ & $\mathrm{~F}$ & $\mathrm{~A} ; \mathrm{B} ; \mathrm{C} ; \mathrm{D} ; \mathrm{E}$ \\
\hline trans-2-Octenal & 151.75 & $\leq 1.00 \mathrm{E}-07$ & $\mathrm{~B} ; \mathrm{C} ; \mathrm{E} ; \mathrm{F}$ & $\mathrm{A} ; \mathrm{C} ; \mathrm{D} ; \mathrm{F}$ & $\mathrm{A} ; \mathrm{B} ; \mathrm{D} ; \mathrm{E} ; \mathrm{F}$ & $\mathrm{B} ; \mathrm{C} ; \mathrm{E} ; \mathrm{F}$ & $\mathrm{A} ; \mathrm{C} ; \mathrm{D} ; \mathrm{F}$ & $\mathrm{A} ; \mathrm{B} ; \mathrm{C} ; \mathrm{D} ; \mathrm{E}$ \\
\hline m-Ethyl-Styrene & 61.93 & $\leq 1.00 \mathrm{E}-07$ & $\mathrm{C} ; \mathrm{D} ; \mathrm{E} ; \mathrm{F}$ & $\mathrm{C} ; \mathrm{D} ; \mathrm{E} ; \mathrm{F}$ & $\mathrm{A} ; \mathrm{B} ; \mathrm{F}$ & $\mathrm{A} ; \mathrm{B} ; \mathrm{F}$ & $\mathrm{A} ; \mathrm{B} ; \mathrm{F}$ & $\mathrm{A} ; \mathrm{B} ; \mathrm{C} ; \mathrm{D} ; \mathrm{E}$ \\
\hline 5-Methyl-2-Heptanone & 124.37 & $\leq 1.00 \mathrm{E}-07$ & $\mathrm{~F}$ & $\mathrm{~F}$ & $\mathrm{~F}$ & $\mathrm{~F}$ & $\mathrm{~F}$ & $\mathrm{~A} ; \mathrm{B} ; \mathrm{C} ; \mathrm{D} ; \mathrm{E}$ \\
\hline 2-Decanone & 61.19 & $\leq 1.00 \mathrm{E}-07$ & $\mathrm{E} ; \mathrm{F}$ & $\mathrm{E} ; \mathrm{F}$ & $\mathrm{E} ; \mathrm{F}$ & $\mathrm{E} ; \mathrm{F}$ & $\mathrm{A} ; \mathrm{B} ; \mathrm{C} ; \mathrm{D} ; \mathrm{F}$ & $\mathrm{A} ; \mathrm{B} ; \mathrm{C} ; \mathrm{D} ; \mathrm{E}$ \\
\hline 1-Octen-3-ol & 47.02 & $\leq 1.00 \mathrm{E}-07$ & $\mathrm{D} ; \mathrm{F}$ & $\mathrm{D} ; \mathrm{F}$ & $\mathrm{D} ; \mathrm{F}$ & $\mathrm{A} ; \mathrm{B} ; \mathrm{C} ; \mathrm{E} ; \mathrm{F}$ & $\mathrm{D} ; \mathrm{F}$ & $\mathrm{A} ; \mathrm{B} ; \mathrm{C} ; \mathrm{D} ; \mathrm{E}$ \\
\hline trans,trans-2,4-Heptadienal & 140.74 & $\leq 1.00 \mathrm{E}-07$ & $\mathrm{~B} ; \mathrm{C} ; \mathrm{D} ; \mathrm{E} ; \mathrm{F}$ & $\mathrm{A} ; \mathrm{C} ; \mathrm{D} ; \mathrm{E} ; \mathrm{F}$ & A; B; D; E; F & $\mathrm{A} ; \mathrm{B} ; \mathrm{C} ; \mathrm{E} ; \mathrm{F}$ & $\mathrm{A} ; \mathrm{B} ; \mathrm{C} ; \mathrm{D} ; \mathrm{F}$ & $\mathrm{A} ; \mathrm{B} ; \mathrm{C} ; \mathrm{D} ; \mathrm{E}$ \\
\hline 1-Heptanol & 187.71 & $\leq 1.00 \mathrm{E}-07$ & $\mathrm{D} ; \mathrm{E} ; \mathrm{F}$ & $\mathrm{D} ; \mathrm{E} ; \mathrm{F}$ & $\mathrm{D} ; \mathrm{E} ; \mathrm{F}$ & $\mathrm{A} ; \mathrm{B} ; \mathrm{C} ; \mathrm{F}$ & $\mathrm{A} ; \mathrm{B} ; \mathrm{C} ; \mathrm{F}$ & $\mathrm{A} ; \mathrm{B} ; \mathrm{C} ; \mathrm{D} ; \mathrm{E}$ \\
\hline cis,cis-3,5-Octadiene & 37.74 & $\leq 1.00 \mathrm{E}-07$ & $\mathrm{C} ; \mathrm{D} ; \mathrm{F}$ & $\mathrm{C} ; \mathrm{D} ; \mathrm{F}$ & $\mathrm{A} ; \mathrm{B} ; \mathrm{E}$ & $\mathrm{A} ; \mathrm{B} ; \mathrm{E}$ & $\mathrm{C} ; \mathrm{D} ; \mathrm{F}$ & $\mathrm{A} ; \mathrm{B} ; \mathrm{E}$ \\
\hline Methyl 2-Oxopropanoate & 91.88 & $\leq 1.00 \mathrm{E}-07$ & $\mathrm{~B} ; \mathrm{C} ; \mathrm{E} ; \mathrm{F}$ & $\mathrm{A} ; \mathrm{C} ; \mathrm{D} ; \mathrm{F}$ & $\mathrm{A} ; \mathrm{B} ; \mathrm{D} ; \mathrm{E} ; \mathrm{F}$ & $\mathrm{B} ; \mathrm{C} ; \mathrm{E} ; \mathrm{F}$ & $\mathrm{A} ; \mathrm{C} ; \mathrm{D} ; \mathrm{F}$ & $\mathrm{A} ; \mathrm{B} ; \mathrm{C} ; \mathrm{D} ; \mathrm{E}$ \\
\hline Dihydromyrcenol & 17.40 & $\leq 1.00 \mathrm{E}-07$ & $\mathrm{~B} ; \mathrm{C} ; \mathrm{E} ; \mathrm{F}$ & $\mathrm{A} ; \mathrm{D}$ & $\mathrm{A} ; \mathrm{D}$ & $\mathrm{B} ; \mathrm{C} ; \mathrm{E} ; \mathrm{F}$ & $\mathrm{A} ; \mathrm{D}$ & $\mathrm{A} ; \mathrm{D}$ \\
\hline trans-Menthone & 252.21 & $\leq 1.00 \mathrm{E}-07$ & $\mathrm{D} ; \mathrm{F}$ & $\mathrm{D} ; \mathrm{F}$ & $\mathrm{D} ; \mathrm{F}$ & $\mathrm{A} ; \mathrm{B} ; \mathrm{C} ; \mathrm{E} ; \mathrm{F}$ & $\mathrm{D} ; \mathrm{F}$ & $\mathrm{A} ; \mathrm{B} ; \mathrm{C} ; \mathrm{D} ; \mathrm{E}$ \\
\hline Furfural & 2.79 & $3.49 \mathrm{E}-02$ & & & $\mathrm{~F}$ & & & $\mathrm{C}$ \\
\hline Ethanoic Acid & 166.51 & $\leq 1.00 \mathrm{E}-07$ & $C ; E ; F$ & $C ; E ; F$ & $\mathrm{~A} ; \mathrm{B} ; \mathrm{D} ; \mathrm{E} ; \mathrm{F}$ & $C ; E ; F$ & $\mathrm{~A} ; \mathrm{B} ; \mathrm{C} ; \mathrm{D} ; \mathrm{F}$ & $\mathrm{A} ; \mathrm{B} ; \mathrm{C} ; \mathrm{D} ; \mathrm{E}$ \\
\hline 2-Ethyl-1-Hexanol & 90.74 & $\leq 1.00 \mathrm{E}-07$ & $\mathrm{~B} ; \mathrm{D} ; \mathrm{E} ; \mathrm{F}$ & $\mathrm{A} ; \mathrm{C} ; \mathrm{D} ; \mathrm{F}$ & $\mathrm{B} ; \mathrm{E} ; \mathrm{F}$ & $\mathrm{A} ; \mathrm{B} ; \mathrm{E} ; \mathrm{F}$ & $\mathrm{A} ; \mathrm{C} ; \mathrm{D} ; \mathrm{F}$ & $\mathrm{A} ; \mathrm{B} ; \mathrm{C} ; \mathrm{D} ; \mathrm{E}$ \\
\hline Decanal & 80.40 & $\leq 1.00 \mathrm{E}-07$ & $\mathrm{~B} ; \mathrm{C} ; \mathrm{D} ; \mathrm{F}$ & $\mathrm{A} ; \mathrm{C} ; \mathrm{D} ; \mathrm{E}$ & $\mathrm{A} ; \mathrm{B} ; \mathrm{E} ; \mathrm{F}$ & $\mathrm{A} ; \mathrm{B} ; \mathrm{E} ; \mathrm{F}$ & $\mathrm{B} ; \mathrm{C} ; \mathrm{D} ; \mathrm{F}$ & $\mathrm{A} ; \mathrm{C} ; \mathrm{D} ; \mathrm{E}$ \\
\hline Furfuryl Formate & 266.49 & $\leq 1.00 \mathrm{E}-07$ & $\mathrm{C} ; \mathrm{D} ; \mathrm{E} ; \mathrm{F}$ & $C ; D ; E ; F$ & A; B; D; E; F & $\mathrm{A} ; \mathrm{B} ; \mathrm{C} ; \mathrm{E} ; \mathrm{F}$ & $\mathrm{A} ; \mathrm{B} ; \mathrm{C} ; \mathrm{D}$ & $\mathrm{A} ; \mathrm{B} ; \mathrm{C} ; \mathrm{D}$ \\
\hline trans-2-Hepten-1-ol & 181.36 & $\leq 1.00 \mathrm{E}-07$ & $\mathrm{C} ; \mathrm{D} ; \mathrm{F}$ & $\mathrm{C} ; \mathrm{D} ; \mathrm{E} ; \mathrm{F}$ & $\mathrm{A} ; \mathrm{B} ; \mathrm{D} ; \mathrm{E} ; \mathrm{F}$ & $\mathrm{A} ; \mathrm{B} ; \mathrm{C} ; \mathrm{E} ; \mathrm{F}$ & $\mathrm{B} ; \mathrm{C} ; \mathrm{D} ; \mathrm{F}$ & $\mathrm{A} ; \mathrm{B} ; \mathrm{C} ; \mathrm{D} ; \mathrm{E}$ \\
\hline 2-Nonanol & 695.37 & $\leq 1.00 \mathrm{E}-07$ & $\mathrm{~B} ; \mathrm{E}$ & $\mathrm{A} ; \mathrm{C} ; \mathrm{D} ; \mathrm{F}$ & $\mathrm{B} ; \mathrm{E} ; \mathrm{F}$ & $\mathrm{B} ; \mathrm{E}$ & $\mathrm{A} ; \mathrm{C} ; \mathrm{D} ; \mathrm{F}$ & $\mathrm{B} ; \mathrm{C} ; \mathrm{E}$ \\
\hline 1-(2-Furanyl)-Ethanone & 560.99 & $\leq 1.00 \mathrm{E}-07$ & $\mathrm{~B} ; \mathrm{C} ; \mathrm{D} ; \mathrm{E} ; \mathrm{F}$ & $\mathrm{A} ; \mathrm{C} ; \mathrm{D} ; \mathrm{E} ; \mathrm{F}$ & $\mathrm{A} ; \mathrm{B}$ & $\mathrm{A} ; \mathrm{B}$ & $\mathrm{A} ; \mathrm{B}$ & $\mathrm{A} ; \mathrm{B}$ \\
\hline 3,5-Octadien-2-one & 101.41 & $\leq 1.00 \mathrm{E}-07$ & $\mathrm{~B} ; \mathrm{C} ; \mathrm{D} ; \mathrm{F}$ & $\mathrm{A} ; \mathrm{C} ; \mathrm{D} ; \mathrm{F}$ & $\mathrm{A} ; \mathrm{B} ; \mathrm{E} ; \mathrm{F}$ & $\mathrm{A} ; \mathrm{B} ; \mathrm{E} ; \mathrm{F}$ & $\mathrm{C} ; \mathrm{D} ; \mathrm{F}$ & $\mathrm{A} ; \mathrm{B} ; \mathrm{C} ; \mathrm{D} ; \mathrm{E}$ \\
\hline
\end{tabular}




\begin{tabular}{|c|c|c|c|c|c|c|c|c|}
\hline Benzaldehyde & 21.35 & $\leq 1.00 \mathrm{E}-07$ & C; D; E; F & $\mathrm{C} ; \mathrm{F}$ & A; B; D; E & $\mathrm{A} ; \mathrm{C} ; \mathrm{F}$ & $\mathrm{A} ; \mathrm{C} ; \mathrm{F}$ & A; B; D; E \\
\hline Furfuryl Acetate & 4.82 & $2.36 \mathrm{E}-03$ & $\mathrm{~F}$ & $\mathrm{~F}$ & & $\mathrm{~F}$ & $\mathrm{~F}$ & $\mathrm{~A} ; \mathrm{B} ; \mathrm{D} ; \mathrm{E}$ \\
\hline Linalool & 384.56 & $\leq 1.00 \mathrm{E}-07$ & $\mathrm{~B} ; \mathrm{C} ; \mathrm{D} ; \mathrm{E} ; \mathrm{F}$ & $\mathrm{A} ; \mathrm{C} ; \mathrm{D} ; \mathrm{E} ; \mathrm{F}$ & $\mathrm{A} ; \mathrm{B} ; \mathrm{D} ; \mathrm{E} ; \mathrm{F}$ & $\mathrm{A} ; \mathrm{B} ; \mathrm{C}$ & $\mathrm{A} ; \mathrm{B} ; \mathrm{C}$ & $\mathrm{A} ; \mathrm{B} ; \mathrm{C}$ \\
\hline$\beta$-Myrcene & 526.99 & $\leq 1.00 \mathrm{E}-07$ & $\mathrm{C} ; \mathrm{D} ; \mathrm{E} ; \mathrm{F}$ & $\mathrm{D} ; \mathrm{F}$ & $\mathrm{A} ; \mathrm{D} ; \mathrm{F}$ & $\mathrm{A} ; \mathrm{B} ; \mathrm{C} ; \mathrm{E} ; \mathrm{F}$ & $\mathrm{A} ; \mathrm{D} ; \mathrm{F}$ & $\mathrm{A} ; \mathrm{B} ; \mathrm{C} ; \mathrm{D} ; \mathrm{E}$ \\
\hline 1-Octanol & 84.42 & $\leq 1.00 \mathrm{E}-07$ & $\mathrm{~B} ; \mathrm{C} ; \mathrm{F}$ & $\mathrm{A} ; \mathrm{C} ; \mathrm{D} ; \mathrm{E} ; \mathrm{F}$ & $\mathrm{A} ; \mathrm{B} ; \mathrm{D} ; \mathrm{E} ; \mathrm{F}$ & $\mathrm{B} ; \mathrm{C} ; \mathrm{F}$ & $\mathrm{B} ; \mathrm{C} ; \mathrm{F}$ & $\mathrm{A} ; \mathrm{B} ; \mathrm{C} ; \mathrm{D} ; \mathrm{E}$ \\
\hline trans-p-2-Menthen-1-ol & 218.39 & $\leq 1.00 \mathrm{E}-07$ & $\mathrm{D} ; \mathrm{F}$ & $\mathrm{D} ; \mathrm{F}$ & $\mathrm{D} ; \mathrm{F}$ & $\mathrm{A} ; \mathrm{B} ; \mathrm{C} ; \mathrm{E} ; \mathrm{F}$ & $\mathrm{D} ; \mathrm{F}$ & $\mathrm{A} ; \mathrm{B} ; \mathrm{C} ; \mathrm{D} ; \mathrm{E}$ \\
\hline Propanoic Acid & 582.46 & $\leq 1.00 \mathrm{E}-07$ & $\mathrm{~B} ; \mathrm{C} ; \mathrm{D} ; \mathrm{E} ; \mathrm{F}$ & $\mathrm{A} ; \mathrm{D} ; \mathrm{F}$ & $\mathrm{A} ; \mathrm{D} ; \mathrm{F}$ & $\mathrm{A} ; \mathrm{B} ; \mathrm{C} ; \mathrm{E} ; \mathrm{F}$ & $\mathrm{A} ; \mathrm{D} ; \mathrm{F}$ & $\mathrm{A} ; \mathrm{B} ; \mathrm{C} ; \mathrm{D} ; \mathrm{E}$ \\
\hline 2,3-Butanediol & 90.14 & $\leq 1.00 \mathrm{E}-07$ & $\mathrm{~B} ; \mathrm{C} ; \mathrm{D} ; \mathrm{E} ; \mathrm{F}$ & $\mathrm{A} ; \mathrm{C} ; \mathrm{D} ; \mathrm{E} ; \mathrm{F}$ & $\mathrm{A} ; \mathrm{B} ; \mathrm{E}$ & $\mathrm{A} ; \mathrm{B} ; \mathrm{E}$ & $\mathrm{A} ; \mathrm{B} ; \mathrm{C} ; \mathrm{D} ; \mathrm{F}$ & $\mathrm{A} ; \mathrm{B} ; \mathrm{E}$ \\
\hline Dimethyl Sulfoxide & 252.84 & $\leq 1.00 \mathrm{E}-07$ & $\mathrm{~B} ; \mathrm{C} ; \mathrm{D} ; \mathrm{F}$ & $\mathrm{A} ; \mathrm{C} ; \mathrm{D} ; \mathrm{E} ; \mathrm{F}$ & $\mathrm{A} ; \mathrm{B} ; \mathrm{D} ; \mathrm{E} ; \mathrm{F}$ & $\mathrm{A} ; \mathrm{B} ; \mathrm{C} ; \mathrm{E} ; \mathrm{F}$ & $\mathrm{B} ; \mathrm{C} ; \mathrm{D} ; \mathrm{F}$ & $\mathrm{A} ; \mathrm{B} ; \mathrm{C} ; \mathrm{D} ; \mathrm{E}$ \\
\hline m-Menth-3-ene & 127.78 & $\leq 1.00 \mathrm{E}-07$ & $\mathrm{C} ; \mathrm{D} ; \mathrm{E} ; \mathrm{F}$ & $\mathrm{C} ; \mathrm{D} ; \mathrm{E} ; \mathrm{F}$ & $\mathrm{A} ; \mathrm{B} ; \mathrm{D}$ & $\mathrm{A} ; \mathrm{B} ; \mathrm{C} ; \mathrm{E} ; \mathrm{F}$ & $\mathrm{A} ; \mathrm{B} ; \mathrm{D}$ & $\mathrm{A} ; \mathrm{B} ; \mathrm{D}$ \\
\hline Trans-(2-Ethylcyclopentyl)-Methanol & 49.21 & $\leq 1.00 \mathrm{E}-07$ & $\mathrm{~B} ; \mathrm{C} ; \mathrm{D} ; \mathrm{E} ; \mathrm{F}$ & $\mathrm{A} ; \mathrm{F}$ & $\mathrm{A} ; \mathrm{F}$ & $\mathrm{A} ; \mathrm{F}$ & $\mathrm{A} ; \mathrm{F}$ & $\mathrm{A} ; \mathrm{B} ; \mathrm{C} ; \mathrm{D} ; \mathrm{E}$ \\
\hline Bornyl Acetate & 119.13 & $\leq 1.00 \mathrm{E}-07$ & $\mathrm{~B} ; \mathrm{F}$ & $\mathrm{A} ; \mathrm{C} ; \mathrm{D} ; \mathrm{E} ; \mathrm{F}$ & $\mathrm{B} ; \mathrm{F}$ & $\mathrm{B} ; \mathrm{E} ; \mathrm{F}$ & $\mathrm{B} ; \mathrm{D} ; \mathrm{F}$ & $\mathrm{A} ; \mathrm{B} ; \mathrm{C} ; \mathrm{D} ; \mathrm{E}$ \\
\hline 4-Cyclopentene-1,3-dione & 365.04 & $\leq 1.00 \mathrm{E}-07$ & $\mathrm{C} ; \mathrm{D} ; \mathrm{E} ; \mathrm{F}$ & $\mathrm{C} ; \mathrm{D} ; \mathrm{E} ; \mathrm{F}$ & $\mathrm{A} ; \mathrm{B} ; \mathrm{D} ; \mathrm{E} ; \mathrm{F}$ & $\mathrm{A} ; \mathrm{B} ; \mathrm{C} ; \mathrm{E} ; \mathrm{F}$ & $\mathrm{A} ; \mathrm{B} ; \mathrm{C} ; \mathrm{D} ; \mathrm{F}$ & $\mathrm{A} ; \mathrm{B} ; \mathrm{C} ; \mathrm{D} ; \mathrm{E}$ \\
\hline Menthol & 1781.56 & $\leq 1.00 \mathrm{E}-07$ & $\mathrm{~B} ; \mathrm{C} ; \mathrm{F}$ & $\mathrm{A} ; \mathrm{C} ; \mathrm{D} ; \mathrm{E} ; \mathrm{F}$ & $\mathrm{A} ; \mathrm{B} ; \mathrm{D} ; \mathrm{E} ; \mathrm{F}$ & $\mathrm{B} ; \mathrm{C} ; \mathrm{F}$ & $\mathrm{B} ; \mathrm{C} ; \mathrm{F}$ & $\mathrm{A} ; \mathrm{B} ; \mathrm{C} ; \mathrm{D} ; \mathrm{E}$ \\
\hline 2-Undecanone & 153.94 & $\leq 1.00 \mathrm{E}-07$ & $\mathrm{~B} ; \mathrm{D} ; \mathrm{E} ; \mathrm{F}$ & $\mathrm{A} ; \mathrm{C} ; \mathrm{D} ; \mathrm{E} ; \mathrm{F}$ & $\mathrm{B} ; \mathrm{D} ; \mathrm{E} ; \mathrm{F}$ & $\mathrm{A} ; \mathrm{B} ; \mathrm{C}$ & $\mathrm{A} ; \mathrm{B} ; \mathrm{C}$ & $\mathrm{A} ; \mathrm{B} ; \mathrm{C}$ \\
\hline 2-(2-Furanyl)-Furan & 82.05 & $\leq 1.00 \mathrm{E}-07$ & $\mathrm{C} ; \mathrm{D} ; \mathrm{E} ; \mathrm{F}$ & $\mathrm{D} ; \mathrm{E} ; \mathrm{F}$ & $\mathrm{A} ; \mathrm{D} ; \mathrm{E} ; \mathrm{F}$ & $\mathrm{A} ; \mathrm{B} ; \mathrm{C} ; \mathrm{E} ; \mathrm{F}$ & $\mathrm{A} ; \mathrm{B} ; \mathrm{C} ; \mathrm{D}$ & $\mathrm{A} ; \mathrm{B} ; \mathrm{C} ; \mathrm{D}$ \\
\hline trans-2-Octen-1-ol & 255.47 & $\leq 1.00 \mathrm{E}-07$ & $\mathrm{~B} ; \mathrm{C} ; \mathrm{D} ; \mathrm{E} ; \mathrm{F}$ & $\mathrm{A} ; \mathrm{C} ; \mathrm{D} ; \mathrm{F}$ & $\mathrm{A} ; \mathrm{B} ; \mathrm{D} ; \mathrm{E} ; \mathrm{F}$ & $\mathrm{A} ; \mathrm{B} ; \mathrm{C} ; \mathrm{E} ; \mathrm{F}$ & $\mathrm{A} ; \mathrm{C} ; \mathrm{D} ; \mathrm{F}$ & $\mathrm{A} ; \mathrm{B} ; \mathrm{C} ; \mathrm{D} ; \mathrm{E}$ \\
\hline trans-2-Nonen-1-ol & 270.13 & $\leq 1.00 \mathrm{E}-07$ & $\mathrm{~B} ; \mathrm{C} ; \mathrm{F}$ & $\mathrm{A} ; \mathrm{C} ; \mathrm{D} ; \mathrm{E} ; \mathrm{F}$ & $\mathrm{A} ; \mathrm{B} ; \mathrm{E} ; \mathrm{F}$ & $\mathrm{B} ; \mathrm{E} ; \mathrm{F}$ & $\mathrm{B} ; \mathrm{C} ; \mathrm{F}$ & $\mathrm{A} ; \mathrm{B} ; \mathrm{C} ; \mathrm{D} ; \mathrm{E}$ \\
\hline Diethylene Glycol Ethyl Ether & 17.81 & $\leq 1.00 \mathrm{E}-07$ & $\mathrm{~B} ; \mathrm{E} ; \mathrm{F}$ & $\mathrm{A} ; \mathrm{C} ; \mathrm{D}$ & $\mathrm{B} ; \mathrm{E}, \mathrm{F}$ & $\mathrm{B} ; \mathrm{E}$ & $\mathrm{A} ; \mathrm{C} ; \mathrm{D} ; \mathrm{F}$ & $\mathrm{A} ; \mathrm{C} ; \mathrm{E}$ \\
\hline 3,5,5-Trimethyl-2-Cyclopenten-1-one & 2.42 & $5.91 \mathrm{E}-02$ & & $\mathrm{D}$ & & B & & \\
\hline cis-p-2-Menthen-1-ol & 4.76 & $2.56 \mathrm{E}-03$ & $\mathrm{D}$ & $\mathrm{D}$ & $\mathrm{D}$ & $\mathrm{A} ; \mathrm{B} ; \mathrm{C} ; \mathrm{E} ; \mathrm{F}$ & $\mathrm{D}$ & $\mathrm{D}$ \\
\hline$\beta$-Cyclocitral & 112.59 & $\leq 1.00 \mathrm{E}-07$ & $\mathrm{C} ; \mathrm{D}, \mathrm{E} ; \mathrm{F}$ & $\mathrm{C} ; \mathrm{D} ; \mathrm{F}$ & $\mathrm{A} ; \mathrm{B} ; \mathrm{E} ; \mathrm{F}$ & $\mathrm{A} ; \mathrm{B} ; \mathrm{E} ; \mathrm{F}$ & $\mathrm{A} ; \mathrm{C} ; \mathrm{E} ; \mathrm{F}$ & $\mathrm{A} ; \mathrm{B} ; \mathrm{C} ; \mathrm{D} ; \mathrm{E}$ \\
\hline Benzeneacetaldehyde & 1009.48 & $\leq 1.00 \mathrm{E}-07$ & $\mathrm{~B} ; \mathrm{C} ; \mathrm{D} ; \mathrm{E} ; \mathrm{F}$ & $\mathrm{A} ; \mathrm{C} ; \mathrm{D} ; \mathrm{E} ; \mathrm{F}$ & $\mathrm{A} ; \mathrm{B} ; \mathrm{E} ; \mathrm{F}$ & $\mathrm{A} ; \mathrm{B} ; \mathrm{F}$ & $\mathrm{A} ; \mathrm{B} ; \mathrm{C} ; \mathrm{F}$ & $\mathrm{A} ; \mathrm{B} ; \mathrm{C} ; \mathrm{D} ; \mathrm{E}$ \\
\hline 1-Nonanol & 298.22 & $\leq 1.00 \mathrm{E}-07$ & $\mathrm{~B} ; \mathrm{E} ; \mathrm{F}$ & $\mathrm{A} ; \mathrm{C} ; \mathrm{D} ; \mathrm{E} ; \mathrm{F}$ & $\mathrm{B} ; \mathrm{E} ; \mathrm{F}$ & $\mathrm{B} ; \mathrm{E} ; \mathrm{F}$ & $\mathrm{A} ; \mathrm{B} ; \mathrm{C} ; \mathrm{D} ; \mathrm{F}$ & $\mathrm{A} ; \mathrm{B} ; \mathrm{C} ; \mathrm{D} ; \mathrm{E}$ \\
\hline Furfuryl Alcohol & 175.76 & $\leq 1.00 \mathrm{E}-07$ & $\mathrm{~B} ; \mathrm{C} ; \mathrm{D} ; \mathrm{E}$ & $\mathrm{A} ; \mathrm{D} ; \mathrm{E} ; \mathrm{F}$ & $\mathrm{A} ; \mathrm{D} ; \mathrm{E} ; \mathrm{F}$ & $\mathrm{A} ; \mathrm{B} ; \mathrm{C} ; \mathrm{E}$ & $\mathrm{A} ; \mathrm{B} ; \mathrm{C} ; \mathrm{D} ; \mathrm{F}$ & $\mathrm{B} ; \mathrm{C} ; \mathrm{E}$ \\
\hline Phoracanthol & 432.85 & $\leq 1.00 \mathrm{E}-07$ & $\mathrm{~B} ; \mathrm{C} ; \mathrm{D} ; \mathrm{E} ; \mathrm{F}$ & $\mathrm{A} ; \mathrm{F}$ & A & $\mathrm{A} ; \mathrm{F}$ & A & $\mathrm{A} ; \mathrm{B} ; \mathrm{D}$ \\
\hline cis-3-Nonen-1-ol & 31.73 & $\leq 1.00 \mathrm{E}-07$ & $\mathrm{~B} ; \mathrm{C} ; \mathrm{E} ; \mathrm{F}$ & $\mathrm{A} ; \mathrm{D} ; \mathrm{E} ; \mathrm{F}$ & $\mathrm{A} ; \mathrm{D} ; \mathrm{E}$ & $\mathrm{B} ; \mathrm{C} ; \mathrm{E} ; \mathrm{F}$ & $\mathrm{A} ; \mathrm{B} ; \mathrm{C} ; \mathrm{D}$ & $\mathrm{A} ; \mathrm{B} ; \mathrm{D}$ \\
\hline cis-Piperitol & 596.97 & $\leq 1.00 \mathrm{E}-07$ & $\mathrm{D} ; \mathrm{F}$ & $\mathrm{D} ; \mathrm{F}$ & $\mathrm{D} ; \mathrm{F}$ & $\mathrm{A} ; \mathrm{B} ; \mathrm{C} ; \mathrm{E} ; \mathrm{F}$ & $\mathrm{D} ; \mathrm{F}$ & $\mathrm{A} ; \mathrm{B} ; \mathrm{C} ; \mathrm{D} ; \mathrm{E}$ \\
\hline p-Methoxy-Styrene & 285.67 & $\leq 1.00 \mathrm{E}-07$ & $\mathrm{~B} ; \mathrm{C} ; \mathrm{E} ; \mathrm{F}$ & $\mathrm{A} ; \mathrm{C} ; \mathrm{D}$ & $\mathrm{A} ; \mathrm{B} ; \mathrm{D} ; \mathrm{E} ; \mathrm{F}$ & $\mathrm{B} ; \mathrm{C} ; \mathrm{E}$ & $\mathrm{A} ; \mathrm{C} ; \mathrm{D} ; \mathrm{F}$ & $\mathrm{A} ; \mathrm{C} ; \mathrm{E}$ \\
\hline (2Z)-3-Ethyl-2,4-Pentadien-1-ol & 165.69 & $\leq 1.00 \mathrm{E}-07$ & $\mathrm{~B} ; \mathrm{C} ; \mathrm{D} ; \mathrm{E} ; \mathrm{F}$ & $\mathrm{A} ; \mathrm{E} ; \mathrm{F}$ & A; E; F & $\mathrm{A} ; \mathrm{E} ; \mathrm{F}$ & $\mathrm{A} ; \mathrm{B} ; \mathrm{C} ; \mathrm{D} ; \mathrm{F}$ & $\mathrm{A} ; \mathrm{B} ; \mathrm{C} ; \mathrm{D} ; \mathrm{B}$ \\
\hline
\end{tabular}




\begin{tabular}{|c|c|c|c|c|c|c|c|c|}
\hline Butylene Glycol Monobutyl Ether & 49.14 & $\leq 1.00 \mathrm{E}-07$ & $\mathrm{C} ; \mathrm{E} ; \mathrm{F}$ & $\mathrm{C} ; \mathrm{E} ; \mathrm{F}$ & $\mathrm{A} ; \mathrm{B} ; \mathrm{D} ; \mathrm{F}$ & $\mathrm{C} ; \mathrm{E} ; \mathrm{F}$ & A; B; D; F & $\mathrm{A} ; \mathrm{B} ; \mathrm{C} ; \mathrm{D} ; \mathrm{E}$ \\
\hline$\alpha$-Terpineol & 250.62 & $\leq 1.00 \mathrm{E}-07$ & C; D; E; F & C; D; E; F & $\mathrm{A} ; \mathrm{B} ; \mathrm{D} ; \mathrm{E} ; \mathrm{F}$ & $\mathrm{A} ; \mathrm{B} ; \mathrm{C} ; \mathrm{F}$ & $\mathrm{A} ; \mathrm{B} ; \mathrm{C} ; \mathrm{F}$ & $\mathrm{A} ; \mathrm{B} ; \mathrm{C} ; \mathrm{D} ; \mathrm{E}$ \\
\hline 1,5-Cyclooctanedione & 6.47 & 3.44E-04 & $\mathrm{F}$ & $\mathrm{F}$ & $\mathrm{F}$ & $\mathrm{F}$ & $\mathrm{F}$ & $\mathrm{A} ; \mathrm{B} ; \mathrm{C} ; \mathrm{D} ; \mathrm{E}$ \\
\hline$\gamma$-Terpinene & 101.61 & $\leq 1.00 \mathrm{E}-07$ & $\mathrm{~B} ; \mathrm{C} ; \mathrm{D} ; \mathrm{E}$ & $\mathrm{A} ; \mathrm{C} ; \mathrm{E} ; \mathrm{F}$ & A; B; D; E; F & $\mathrm{A} ; \mathrm{C} ; \mathrm{E} ; \mathrm{F}$ & $\mathrm{A} ; \mathrm{B} ; \mathrm{C} ; \mathrm{D} ; \mathrm{F}$ & $\mathrm{B} ; \mathrm{C} ; \mathrm{D} ; \mathrm{E}$ \\
\hline trans-2-Decenol & 127.16 & $\leq 1.00 \mathrm{E}-07$ & $\mathrm{C} ; \mathrm{D} ; \mathrm{F}$ & $\mathrm{C} ; \mathrm{D} ; \mathrm{F}$ & $\mathrm{A} ; \mathrm{B} ; \mathrm{E} ; \mathrm{F}$ & $\mathrm{A} ; \mathrm{B} ; \mathrm{E} ; \mathrm{F}$ & $\mathrm{C} ; \mathrm{D} ; \mathrm{F}$ & $\mathrm{A} ; \mathrm{B} ; \mathrm{C} ; \mathrm{D} ; \mathrm{E}$ \\
\hline 2-Propenoic Acid & 107.28 & $\leq 1.00 \mathrm{E}-07$ & $\mathrm{~B} ; \mathrm{C} ; \mathrm{D} ; \mathrm{E} ; \mathrm{F}$ & A & A & A & A & A \\
\hline 2-Undecanol & 1153.59 & $\leq 1.00 \mathrm{E}-07$ & $\mathrm{~B} ; \mathrm{E}$ & $\mathrm{A} ; \mathrm{C} ; \mathrm{D} ; \mathrm{E} ; \mathrm{F}$ & $\mathrm{B} ; \mathrm{E}$ & $\mathrm{B} ; \mathrm{E}$ & $\mathrm{A} ; \mathrm{B} ; \mathrm{C} ; \mathrm{D} ; \mathrm{F}$ & $\mathrm{B} ; \mathrm{E}$ \\
\hline 3-Ethyl-Benzaldehyde & 36.39 & $\leq 1.00 \mathrm{E}-07$ & $\mathrm{~B} ; \mathrm{E} ; \mathrm{F}$ & $\mathrm{A} ; \mathrm{C} ; \mathrm{E} ; \mathrm{F}$ & $\mathrm{B} ; \mathrm{E}$ & $\mathrm{E} ; \mathrm{F}$ & $\mathrm{A} ; \mathrm{B} ; \mathrm{C} ; \mathrm{D} ; \mathrm{F}$ & $\mathrm{A} ; \mathrm{B} ; \mathrm{D} ; \mathrm{E}$ \\
\hline 3-Hydroxy-Benzaldehyde & 90.20 & $\leq 1.00 \mathrm{E}-07$ & $\mathrm{~B} ; \mathrm{C} ; \mathrm{D} ; \mathrm{E} ; \mathrm{F}$ & $\mathrm{A} ; \mathrm{D}$ & $\mathrm{A} ; \mathrm{D} ; \mathrm{E} ; \mathrm{F}$ & $\mathrm{A} ; \mathrm{B} ; \mathrm{C} ; \mathrm{E} ; \mathrm{F}$ & $\mathrm{A}, \mathrm{C} ; \mathrm{D}$ & $\mathrm{A}, \mathrm{C} ; \mathrm{D}$ \\
\hline trans-2-Nonene & 293.65 & $\leq 1.00 \mathrm{E}-07$ & $\mathrm{~B} ; \mathrm{C} ; \mathrm{D} ; \mathrm{E} ; \mathrm{F}$ & A; C; D; E; F & $\mathrm{A} ; \mathrm{B} ; \mathrm{D} ; \mathrm{E} ; \mathrm{F}$ & $\mathrm{A} ; \mathrm{B} ; \mathrm{C}$ & $\mathrm{A} ; \mathrm{B} ; \mathrm{C} ; \mathrm{F}$ & $\mathrm{A} ; \mathrm{B} ; \mathrm{C} ; \mathrm{E}$ \\
\hline 2-Methyl-2-Pentenal & 86.36 & $\leq 1.00 \mathrm{E}-07$ & $\mathrm{~B} ; \mathrm{C} ; \mathrm{D} ; \mathrm{E} ; \mathrm{F}$ & $\mathrm{A} ; \mathrm{D} ; \mathrm{E} ; \mathrm{F}$ & $\mathrm{A} ; \mathrm{D} ; \mathrm{E} ; \mathrm{F}$ & $\mathrm{A} ; \mathrm{B} ; \mathrm{C} ; \mathrm{E}$ & $\mathrm{A} ; \mathrm{B} ; \mathrm{C} ; \mathrm{D} ; \mathrm{F}$ & $\mathrm{A} ; \mathrm{B} ; \mathrm{C} ; \mathrm{E}$ \\
\hline cis-5-Decenol & 706.67 & $\leq 1.00 \mathrm{E}-07$ & $\mathrm{~B} ; \mathrm{E} ; \mathrm{F}$ & $\mathrm{A} ; \mathrm{C} ; \mathrm{D} ; \mathrm{E} ; \mathrm{F}$ & $\mathrm{B} ; \mathrm{E} ; \mathrm{F}$ & $\mathrm{B} ; \mathrm{E} ; \mathrm{F}$ & $\mathrm{A} ; \mathrm{B} ; \mathrm{C} ; \mathrm{D} ; \mathrm{F}$ & $\mathrm{A} ; \mathrm{B} ; \mathrm{C} ; \mathrm{D} ; \mathrm{E}$ \\
\hline trans-Piperitol & 481.52 & $\leq 1.00 \mathrm{E}-07$ & $\mathrm{~B} ; \mathrm{D} ; \mathrm{F}$ & $\mathrm{A} ; \mathrm{C} ; \mathrm{D} ; \mathrm{E} ; \mathrm{F}$ & $\mathrm{B} ; \mathrm{D} ; \mathrm{F}$ & $\mathrm{A} ; \mathrm{B} ; \mathrm{C} ; \mathrm{E} ; \mathrm{F}$ & $\mathrm{B} ; \mathrm{D} ; \mathrm{F}$ & $\mathrm{A} ; \mathrm{B} ; \mathrm{C} ; \mathrm{D} ; \mathrm{E}$ \\
\hline 4-Ethyl-Benzaldehyde & 56.90 & $\leq 1.00 \mathrm{E}-07$ & $\mathrm{~B} ; \mathrm{C} ; \mathrm{F}$ & $\mathrm{A} ; \mathrm{C} ; \mathrm{F}$ & $\mathrm{A} ; \mathrm{B} ; \mathrm{D} ; \mathrm{E} ; \mathrm{F}$ & $\mathrm{C} ; \mathrm{F}$ & $\mathrm{C} ; \mathrm{F}$ & $\mathrm{A} ; \mathrm{B} ; \mathrm{C} ; \mathrm{D} ; \mathrm{E}$ \\
\hline Naphthalene & 149.55 & $\leq 1.00 \mathrm{E}-07$ & $\mathrm{~B} ; \mathrm{C} ; \mathrm{E} ; \mathrm{F}$ & $\mathrm{A} ; \mathrm{C} ; \mathrm{D} ; \mathrm{F}$ & $\mathrm{A} ; \mathrm{B} ; \mathrm{D} ; \mathrm{E} ; \mathrm{F}$ & $\mathrm{B} ; \mathrm{C} ; \mathrm{D} ; \mathrm{F}$ & $\mathrm{A} ; \mathrm{C} ; \mathrm{D} ; \mathrm{F}$ & $\mathrm{A} ; \mathrm{B} ; \mathrm{C} ; \mathrm{D} ; \mathrm{E}$ \\
\hline 1-Decanol & 52.83 & $\leq 1.00 \mathrm{E}-07$ & $\mathrm{~B} ; \mathrm{C} ; \mathrm{D} ; \mathrm{E} ; \mathrm{F}$ & A & A & $\mathrm{A} ; \mathrm{E}$ & $\mathrm{A} ; \mathrm{D}$ & A \\
\hline Cyclodecane & 97.31 & $\leq 1.00 \mathrm{E}-07$ & $\mathrm{~B} ; \mathrm{C} ; \mathrm{D} ; \mathrm{E} ; \mathrm{F}$ & $\mathrm{A} ; \mathrm{C} ; \mathrm{D} ; \mathrm{E} ; \mathrm{F}$ & A; B; E; F & $\mathrm{A} ; \mathrm{B} ; \mathrm{E} ; \mathrm{F}$ & $\mathrm{A} ; \mathrm{B} ; \mathrm{C} ; \mathrm{D}$ & $\mathrm{A} ; \mathrm{B} ; \mathrm{C} ; \mathrm{D}$ \\
\hline trans,trans-2,4-Decadienal & 216.56 & $\leq 1.00 \mathrm{E}-07$ & $\mathrm{~B} ; \mathrm{C} ; \mathrm{E} ; \mathrm{F}$ & $\mathrm{A} ; \mathrm{C} ; \mathrm{F}$ & $\mathrm{A} ; \mathrm{B} ; \mathrm{D} ; \mathrm{E} ; \mathrm{F}$ & $\mathrm{C} ; \mathrm{F}$ & $\mathrm{A} ; \mathrm{C} ; \mathrm{F}$ & $\mathrm{A} ; \mathrm{B} ; \mathrm{C} ; \mathrm{D} ; \mathrm{E}$ \\
\hline 1,2-Cyclopentanedione & 237.96 & $\leq 1.00 \mathrm{E}-07$ & $\mathrm{~B} ; \mathrm{C} ; \mathrm{E} ; \mathrm{F}$ & $\mathrm{A} ; \mathrm{C} ; \mathrm{D} ; \mathrm{E} ; \mathrm{F}$ & $\mathrm{A} ; \mathrm{B} ; \mathrm{D}$ & $\mathrm{B} ; \mathrm{C} ; \mathrm{E} ; \mathrm{F}$ & $\mathrm{A} ; \mathrm{B} ; \mathrm{D}$ & $\mathrm{A} ; \mathrm{B} ; \mathrm{D}$ \\
\hline Cyclodecene & 176.38 & $\leq 1.00 \mathrm{E}-07$ & C; D; E; F & $\mathrm{C} ; \mathrm{D} ; \mathrm{F}$ & $\mathrm{A} ; \mathrm{B} ; \mathrm{D} ; \mathrm{F}$ & $\mathrm{A} ; \mathrm{B} ; \mathrm{C} ; \mathrm{E} ; \mathrm{F}$ & $\mathrm{A} ; \mathrm{D} ; \mathrm{F}$ & $\mathrm{A} ; \mathrm{B} ; \mathrm{C} ; \mathrm{D} ; \mathrm{E}$ \\
\hline cis-4-Decenol & 327.97 & $\leq 1.00 \mathrm{E}-07$ & B; C; D; E; F & $\mathrm{A} ; \mathrm{C} ; \mathrm{D} ; \mathrm{E}$ & $\mathrm{A} ; \mathrm{B} ; \mathrm{E} ; \mathrm{F}$ & $\mathrm{A} ; \mathrm{B} ; \mathrm{E} ; \mathrm{F}$ & $\mathrm{A} ; \mathrm{B} ; \mathrm{C} ; \mathrm{D} ; \mathrm{F}$ & $\mathrm{A} ; \mathrm{C} ; \mathrm{D} ; \mathrm{E}$ \\
\hline 1-Phenyl-2-Butanone & 112.81 & $\leq 1.00 \mathrm{E}-07$ & $\mathrm{C} ; \mathrm{E}$ & $\mathrm{C} ; \mathrm{E}$ & $\mathrm{A} ; \mathrm{B} ; \mathrm{D} ; \mathrm{E} ; \mathrm{F}$ & $C ; E ; F$ & $\mathrm{~A} ; \mathrm{B} ; \mathrm{C} ; \mathrm{D} ; \mathrm{F}$ & $C ; D ; E$ \\
\hline Phenethyl Alcohol & 246.86 & $\leq 1.00 \mathrm{E}-07$ & $\mathrm{~B} ; \mathrm{C} ; \mathrm{D} ; \mathrm{E} ; \mathrm{F}$ & $\mathrm{A} ; \mathrm{C} ; \mathrm{D} ; \mathrm{E} ; \mathrm{F}$ & $\mathrm{A} ; \mathrm{B} ; \mathrm{D} ; \mathrm{F}$ & $\mathrm{A} ; \mathrm{B} ; \mathrm{C} ; \mathrm{E} ; \mathrm{F}$ & $\mathrm{A} ; \mathrm{B} ; \mathrm{D} ; \mathrm{F}$ & $\mathrm{A} ; \mathrm{B} ; \mathrm{C} ; \mathrm{D} ; \mathrm{E}$ \\
\hline trans,cis-2,4-Decadienal & 223.31 & $\leq 1.00 \mathrm{E}-07$ & $\mathrm{~B} ; \mathrm{C} ; \mathrm{D} ; \mathrm{E} ; \mathrm{F}$ & $\mathrm{A} ; \mathrm{C} ; \mathrm{F}$ & $\mathrm{A} ; \mathrm{B} ; \mathrm{D} ; \mathrm{E} ; \mathrm{F}$ & $\mathrm{A} ; \mathrm{C} ; \mathrm{F}$ & $\mathrm{A} ; \mathrm{C} ; \mathrm{F}$ & $\mathrm{A} ; \mathrm{B} ; \mathrm{C} ; \mathrm{D} ; \mathrm{E}$ \\
\hline 2-Cyclohexenol & 443.59 & $\leq 1.00 \mathrm{E}-07$ & $\mathrm{~B} ; \mathrm{C} ; \mathrm{D} ; \mathrm{E} ; \mathrm{F}$ & $\mathrm{A} ; \mathrm{C} ; \mathrm{D} ; \mathrm{E} ; \mathrm{F}$ & $\mathrm{A} ; \mathrm{B} ; \mathrm{D} ; \mathrm{E}$ & $\mathrm{A} ; \mathrm{B} ; \mathrm{C} ; \mathrm{E} ; \mathrm{F}$ & $\mathrm{A} ; \mathrm{B} ; \mathrm{C} ; \mathrm{D}$ & $\mathrm{A} ; \mathrm{B} ; \mathrm{D}$ \\
\hline 2-Ethyl Hexanoic Acid & 264.43 & $\leq 1.00 \mathrm{E}-07$ & $\mathrm{~B} ; \mathrm{C} ; \mathrm{D} ; \mathrm{E} ; \mathrm{F}$ & $\mathrm{A} ; \mathrm{C} ; \mathrm{D} ; \mathrm{F}$ & $\mathrm{A} ; \mathrm{B} ; \mathrm{D} ; \mathrm{E} ; \mathrm{F}$ & $\mathrm{A} ; \mathrm{B} ; \mathrm{C} ; \mathrm{E}$ & $\mathrm{A} ; \mathrm{C} ; \mathrm{D} ; \mathrm{F}$ & $\mathrm{A} ; \mathrm{B} ; \mathrm{C} ; \mathrm{E}$ \\
\hline 3-Methyl-1,2-Cyclopentanedione & 544.81 & $\leq 1.00 \mathrm{E}-07$ & $\mathrm{~B} ; \mathrm{C} ; \mathrm{D} ; \mathrm{E} ; \mathrm{F}$ & $\mathrm{A} ; \mathrm{C} ; \mathrm{D} ; \mathrm{E} ; \mathrm{F}$ & $\mathrm{A} ; \mathrm{B} ; \mathrm{D} ; \mathrm{E}$ & $\mathrm{A} ; \mathrm{B} ; \mathrm{C} ; \mathrm{E} ; \mathrm{F}$ & $\mathrm{A} ; \mathrm{B} ; \mathrm{C} ; \mathrm{D}$ & $\mathrm{A} ; \mathrm{B} ; \mathrm{D}$ \\
\hline trans-Cinnamaldehyde & 60.02 & $\leq 1.00 \mathrm{E}-07$ & $\mathrm{~B} ; \mathrm{C} ; \mathrm{D} ; \mathrm{E} ; \mathrm{F}$ & $\mathrm{A} ; \mathrm{C} ; \mathrm{D} ; \mathrm{F}$ & $\mathrm{A} ; \mathrm{B} ; \mathrm{D}$ & A; B; E; F & $\mathrm{A} ; \mathrm{C} ; \mathrm{D} ; \mathrm{F}$ & A; B; D; E \\
\hline Heptanoic Acid & 289.77 & $\leq 1.00 \mathrm{E}-07$ & $\mathrm{~B} ; \mathrm{D} ; \mathrm{E} ; \mathrm{F}$ & $\mathrm{A} ; \mathrm{C} ; \mathrm{D} ; \mathrm{E} ; \mathrm{F}$ & $\mathrm{B} ; \mathrm{D} ; \mathrm{E} ; \mathrm{F}$ & $\mathrm{A} ; \mathrm{B} ; \mathrm{C} ; \mathrm{E} ; \mathrm{F}$ & $\mathrm{A} ; \mathrm{B} ; \mathrm{C} ; \mathrm{D} ; \mathrm{F}$ & $\mathrm{A} ; \mathrm{B} ; \mathrm{C} ; \mathrm{D} ; \mathrm{E}$ \\
\hline Mequinol & 105.13 & $\leq 1.00 \mathrm{E}-07$ & $\mathrm{C} ; \mathrm{D} ; \mathrm{E} ; \mathrm{F}$ & $\mathrm{C} ; \mathrm{D} ; \mathrm{E}$ & $\mathrm{A} ; \mathrm{B} ; \mathrm{D} ; \mathrm{E} ; \mathrm{F}$ & $\mathrm{A} ; \mathrm{B} ; \mathrm{C} ; \mathrm{E} ; \mathrm{F}$ & $\mathrm{A} ; \mathrm{B} ; \mathrm{C} ; \mathrm{D} ; \mathrm{F}$ & $\mathrm{A} ; \mathrm{C} ; \mathrm{D} ; \mathrm{E}$ \\
\hline
\end{tabular}




\begin{tabular}{|c|c|c|c|c|c|c|c|c|}
\hline 2,4,5-Trihydroxypyrimidine & 637.12 & $\leq 1.00 \mathrm{E}-07$ & $\mathrm{~B} ; \mathrm{C} ; \mathrm{D} ; \mathrm{E} ; \mathrm{F}$ & $\mathrm{A} ; \mathrm{D}$ & $\mathrm{A} ; \mathrm{F}$ & $\mathrm{A} ; \mathrm{B} ; \mathrm{E} ; \mathrm{F}$ & $\mathrm{A} ; \mathrm{D}$ & $\mathrm{A}, \mathrm{C} ; \mathrm{D}$ \\
\hline cis-Cinnamaldehyde & 74.15 & $\leq 1.00 \mathrm{E}-07$ & $\mathrm{C} ; \mathrm{E}$ & $\mathrm{C} ; \mathrm{E}$ & $\mathrm{A} ; \mathrm{B} ; \mathrm{D} ; \mathrm{E} ; \mathrm{F}$ & $\mathrm{C} ; \mathrm{E}$ & $\mathrm{A} ; \mathrm{B} ; \mathrm{C} ; \mathrm{D} ; \mathrm{F}$ & $\mathrm{C} ; \mathrm{E}$ \\
\hline Benzenemethanol & 99.47 & $\leq 1.00 \mathrm{E}-07$ & $\mathrm{~B} ; \mathrm{C} ; \mathrm{D} ; \mathrm{F}$ & $\mathrm{A} ; \mathrm{C} ; \mathrm{D}$ & A; B; D; E; F & $\mathrm{A} ; \mathrm{B} ; \mathrm{C} ; \mathrm{E} ; \mathrm{F}$ & $\mathrm{C} ; \mathrm{D} ; \mathrm{F}$ & $\mathrm{A} ; \mathrm{C} ; \mathrm{D} ; \mathrm{E}$ \\
\hline Mesitaldehyde & 73.47 & $\leq 1.00 \mathrm{E}-07$ & $\mathrm{~B} ; \mathrm{E}$ & $\mathrm{A} ; \mathrm{C} ; \mathrm{D} ; \mathrm{E} ; \mathrm{F}$ & $\mathrm{B} ; \mathrm{E}$ & $\mathrm{B} ; \mathrm{E}$ & $\mathrm{A} ; \mathrm{B} ; \mathrm{C} ; \mathrm{D} ; \mathrm{F}$ & $\mathrm{B} ; \mathrm{E}$ \\
\hline 2-Ethylhexyl Octanoate & 873.20 & $\leq 1.00 \mathrm{E}-07$ & $\mathrm{~B} ; \mathrm{C} ; \mathrm{D} ; \mathrm{E} ; \mathrm{F}$ & $\mathrm{A} ; \mathrm{C} ; \mathrm{D} ; \mathrm{E} ; \mathrm{F}$ & A; B; D; E; F & $\mathrm{A} ; \mathrm{B} ; \mathrm{C} ; \mathrm{E} ; \mathrm{F}$ & $\mathrm{A} ; \mathrm{B} ; \mathrm{C} ; \mathrm{D}$ & $\mathrm{A} ; \mathrm{B} ; \mathrm{C} ; \mathrm{D}$ \\
\hline Benzeneethanol & 109.24 & $\leq 1.00 \mathrm{E}-07$ & B; C; D; E; F & $\mathrm{A} ; \mathrm{C} ; \mathrm{D} ; \mathrm{F}$ & $\mathrm{A} ; \mathrm{B} ; \mathrm{D} ; \mathrm{E}$ & $\mathrm{A} ; \mathrm{B} ; \mathrm{C} ; \mathrm{E} ; \mathrm{F}$ & $\mathrm{A} ; \mathrm{C} ; \mathrm{D} ; \mathrm{F}$ & $\mathrm{A} ; \mathrm{B} ; \mathrm{D} ; \mathrm{E}$ \\
\hline 1,4-Butanediol & 76.56 & $\leq 1.00 \mathrm{E}-07$ & $\mathrm{~B} ; \mathrm{C} ; \mathrm{D} ; \mathrm{E} ; \mathrm{F}$ & $\mathrm{A} ; \mathrm{C} ; \mathrm{E} ; \mathrm{F}$ & $\mathrm{A} ; \mathrm{B} ; \mathrm{E}$ & $\mathrm{A} ; \mathrm{E} ; \mathrm{F}$ & $\mathrm{A} ; \mathrm{B} ; \mathrm{C} ; \mathrm{D} ; \mathrm{F}$ & $\mathrm{A} ; \mathrm{B} ; \mathrm{D} ; \mathrm{E}$ \\
\hline$\beta$-Ionone & 116.48 & $\leq 1.00 \mathrm{E}-07$ & $\mathrm{C} ; \mathrm{D} ; \mathrm{F}$ & $\mathrm{C} ; \mathrm{D} ; \mathrm{E} ; \mathrm{F}$ & A; B; D; E; F & $\mathrm{A} ; \mathrm{B} ; \mathrm{C} ; \mathrm{E} ; \mathrm{F}$ & $\mathrm{B} ; \mathrm{C} ; \mathrm{D} ; \mathrm{F}$ & $\mathrm{A} ; \mathrm{B} ; \mathrm{C} ; \mathrm{D} ; \mathrm{E}$ \\
\hline 2-Methyl-1,4-Benzenediol & 80.72 & $\leq 1.00 \mathrm{E}-07$ & $\mathrm{~B} ; \mathrm{C} ; \mathrm{E} ; \mathrm{F}$ & $\mathrm{A} ; \mathrm{C} ; \mathrm{D} ; \mathrm{E} ; \mathrm{F}$ & $\mathrm{A} ; \mathrm{B} ; \mathrm{D}$ & $\mathrm{B} ; \mathrm{C} ; \mathrm{E} ; \mathrm{F}$ & $\mathrm{A} ; \mathrm{B} ; \mathrm{D}$ & $\mathrm{A} ; \mathrm{B} ; \mathrm{D}$ \\
\hline 2-Ethyl Heptanoic Acid & 85.33 & $\leq 1.00 \mathrm{E}-07$ & $\mathrm{~B} ; \mathrm{E}$ & $\mathrm{A} ; \mathrm{C} ; \mathrm{D} ; \mathrm{E} ; \mathrm{F}$ & $\mathrm{B} ; \mathrm{E}$ & $\mathrm{B} ; \mathrm{E}$ & $\mathrm{A} ; \mathrm{B} ; \mathrm{C} ; \mathrm{D} ; \mathrm{F}$ & $\mathrm{B} ; \mathrm{E}$ \\
\hline 1-Dodecanol & 64.66 & $\leq 1.00 \mathrm{E}-07$ & $\mathrm{~B} ; \mathrm{D} ; \mathrm{E}$ & $\mathrm{A} ; \mathrm{C} ; \mathrm{D} ; \mathrm{F}$ & $\mathrm{B} ; \mathrm{D} ; \mathrm{E}$ & $\mathrm{A} ; \mathrm{B} ; \mathrm{C} ; \mathrm{E} ; \mathrm{F}$ & $\mathrm{A} ; \mathrm{C} ; \mathrm{D} ; \mathrm{F}$ & $\mathrm{B} ; \mathrm{D} ; \mathrm{E}$ \\
\hline Benzalacetone & 246.67 & $\leq 1.00 \mathrm{E}-07$ & $\mathrm{~B} ; \mathrm{C} ; \mathrm{D} ; \mathrm{E} ; \mathrm{F}$ & $\mathrm{A} ; \mathrm{C} ; \mathrm{E} ; \mathrm{F}$ & $\mathrm{A} ; \mathrm{B} ; \mathrm{E}$ & $\mathrm{A} ; \mathrm{E} ; \mathrm{F}$ & $\mathrm{A} ; \mathrm{B} ; \mathrm{C} ; \mathrm{D} ; \mathrm{F}$ & $\mathrm{A} ; \mathrm{B} ; \mathrm{D} ; \mathrm{E}$ \\
\hline Maltol & 71.61 & $\leq 1.00 \mathrm{E}-07$ & $\mathrm{C} ; \mathrm{D} ; \mathrm{E} ; \mathrm{F}$ & $\mathrm{C} ; \mathrm{D} ; \mathrm{E} ; \mathrm{F}$ & $\mathrm{A} ; \mathrm{B} ; \mathrm{D} ; \mathrm{E}$ & $\mathrm{A} ; \mathrm{B} ; \mathrm{C} ; \mathrm{E} ; \mathrm{F}$ & $\mathrm{A} ; \mathrm{B} ; \mathrm{C} ; \mathrm{D} ; \mathrm{F}$ & $\mathrm{A} ; \mathrm{B} ; \mathrm{D}, \mathrm{E}$ \\
\hline 2-Acetylpyrrole & 1508.90 & $\leq 1.00 \mathrm{E}-07$ & $\mathrm{~B} ; \mathrm{C} ; \mathrm{D} ; \mathrm{E} ; \mathrm{F}$ & $\mathrm{A} ; \mathrm{C} ; \mathrm{D} ; \mathrm{E} ; \mathrm{F}$ & $\mathrm{A} ; \mathrm{B} ; \mathrm{D} ; \mathrm{F}$ & $\mathrm{A} ; \mathrm{B} ; \mathrm{C} ; \mathrm{E} ; \mathrm{F}$ & $\mathrm{A} ; \mathrm{B} ; \mathrm{D} ; \mathrm{F}$ & $\mathrm{A} ; \mathrm{B} ; \mathrm{C} ; \mathrm{D} ; \mathrm{E}$ \\
\hline 2,4-Decadien-1-ol & 35.84 & $\leq 1.00 \mathrm{E}-07$ & $\mathrm{~B} ; \mathrm{C} ; \mathrm{F}$ & $\mathrm{A} ; \mathrm{F}$ & $\mathrm{A} ; \mathrm{F}$ & $\mathrm{F}$ & $\mathrm{F}$ & $\mathrm{A} ; \mathrm{B} ; \mathrm{C} ; \mathrm{D} ; \mathrm{E}$ \\
\hline Levoglucosenone & 154.19 & $\leq 1.00 \mathrm{E}-07$ & B; D; E; F & $\mathrm{A} ; \mathrm{C} ; \mathrm{E} ; \mathrm{F}$ & $\mathrm{B} ; \mathrm{D} ; \mathrm{E} ; \mathrm{F}$ & $\mathrm{A} ; \mathrm{C} ; \mathrm{E} ; \mathrm{F}$ & $\mathrm{A} ; \mathrm{B} ; \mathrm{C} ; \mathrm{D} ; \mathrm{F}$ & $\mathrm{A} ; \mathrm{B} ; \mathrm{C} ; \mathrm{D} ; \mathrm{E}$ \\
\hline Phenol & 26.92 & $\leq 1.00 \mathrm{E}-07$ & $\mathrm{C} ; \mathrm{D}$ & $\mathrm{C} ; \mathrm{D}$ & A; B; D; E; F & $\mathrm{A} ; \mathrm{B} ; \mathrm{C} ; \mathrm{E} ; \mathrm{F}$ & $\mathrm{C} ; \mathrm{D}$ & $\mathrm{C} ; \mathrm{D}$ \\
\hline (2-Furanyl)-2-Hydroxy-Ethanone & 356.33 & $\leq 1.00 \mathrm{E}-07$ & $\mathrm{~B} ; \mathrm{C} ; \mathrm{D} ; \mathrm{E} ; \mathrm{F}$ & $\mathrm{A} ; \mathrm{C} ; \mathrm{D} ; \mathrm{F}$ & $\mathrm{A} ; \mathrm{B} ; \mathrm{D} ; \mathrm{E}$ & $\mathrm{A} ; \mathrm{B} ; \mathrm{C} ; \mathrm{E}$ & $\mathrm{A} ; \mathrm{C} ; \mathrm{D} ; \mathrm{F}$ & $\mathrm{A} ; \mathrm{B} ; \mathrm{E}$ \\
\hline 4,6-Dimethyl-Dodecane & 274.61 & $\leq 1.00 \mathrm{E}-07$ & $\mathrm{~B} ; \mathrm{C} ; \mathrm{D} ; \mathrm{E} ; \mathrm{F}$ & $\mathrm{A} ; \mathrm{C} ; \mathrm{E} ; \mathrm{F}$ & $\mathrm{A} ; \mathrm{B} ; \mathrm{D} ; \mathrm{F}$ & $\mathrm{A} ; \mathrm{C} ; \mathrm{E} ; \mathrm{F}$ & $\mathrm{A} ; \mathrm{B} ; \mathrm{D} ; \mathrm{F}$ & $\mathrm{A} ; \mathrm{B} ; \mathrm{C} ; \mathrm{D} ; \mathrm{E}$ \\
\hline Ethyl Decanoate & 215.54 & $\leq 1.00 \mathrm{E}-07$ & $C ; E ; F$ & $\mathrm{D} ; \mathrm{E} ; \mathrm{F}$ & $\mathrm{A} ; \mathrm{D} ; \mathrm{E} ; \mathrm{F}$ & $\mathrm{B} ; \mathrm{C} ; \mathrm{D} ; \mathrm{F}$ & $\mathrm{A} ; \mathrm{B} ; \mathrm{C} ; \mathrm{D} ; \mathrm{F}$ & $\mathrm{A} ; \mathrm{B} ; \mathrm{C} ; \mathrm{D} ; \mathrm{E}$ \\
\hline 1-Methyl-4-Piperidinone & 558.99 & $\leq 1.00 \mathrm{E}-07$ & $\mathrm{~B} ; \mathrm{C} ; \mathrm{E} ; \mathrm{F}$ & $\mathrm{A} ; \mathrm{D} ; \mathrm{F}$ & $\mathrm{A} ; \mathrm{D} ; \mathrm{E} ; \mathrm{F}$ & $\mathrm{B} ; \mathrm{C} ; \mathrm{E} ; \mathrm{F}$ & $\mathrm{A} ; \mathrm{C} ; \mathrm{D} ; \mathrm{F}$ & $\mathrm{A} ; \mathrm{B} ; \mathrm{C} ; \mathrm{D} ; \mathrm{E}$ \\
\hline 2,2-Dimethyl-3-Heptanone & 65.10 & $\leq 1.00 \mathrm{E}-07$ & $\mathrm{~B} ; \mathrm{D} ; \mathrm{F}$ & $\mathrm{A} ; \mathrm{C} ; \mathrm{D} ; \mathrm{E} ; \mathrm{F}$ & $\mathrm{B} ; \mathrm{D} ; \mathrm{F}$ & $\mathrm{A} ; \mathrm{B} ; \mathrm{C} ; \mathrm{E} ; \mathrm{F}$ & $\mathrm{B} ; \mathrm{D} ; \mathrm{F}$ & $\mathrm{A} ; \mathrm{B} ; \mathrm{C} ; \mathrm{D} ; \mathrm{E}$ \\
\hline Octanoic Acid & 113.84 & $\leq 1.00 \mathrm{E}-07$ & $\mathrm{~B} ; \mathrm{C} ; \mathrm{D} ; \mathrm{E} ; \mathrm{F}$ & $\mathrm{A} ; \mathrm{C} ; \mathrm{D} ; \mathrm{F}$ & $\mathrm{A} ; \mathrm{B} ; \mathrm{E}$ & $\mathrm{A} ; \mathrm{B} ; \mathrm{E}$ & $\mathrm{A} ; \mathrm{C} ; \mathrm{D} ; \mathrm{F}$ & $\mathrm{A} ; \mathrm{B} ; \mathrm{E}$ \\
\hline 1,3-Dihydroxy-2-Propanone & 198.54 & $\leq 1.00 \mathrm{E}-07$ & $\mathrm{~B} ; \mathrm{C} ; \mathrm{D} ; \mathrm{E} ; \mathrm{F}$ & $\mathrm{A} ; \mathrm{C} ; \mathrm{D} ; \mathrm{E} ; \mathrm{F}$ & $\mathrm{A} ; \mathrm{B} ; \mathrm{D}$ & $\mathrm{A} ; \mathrm{B} ; \mathrm{C} ; \mathrm{E} ; \mathrm{F}$ & $\mathrm{A} ; \mathrm{B} ; \mathrm{D}$ & $\mathrm{A} ; \mathrm{B} ; \mathrm{D}$ \\
\hline 2-Phenoxy-Ethanol & 592.27 & $\leq 1.00 \mathrm{E}-07$ & $\mathrm{C} ; \mathrm{D} ; \mathrm{E} ; \mathrm{F}$ & $\mathrm{C} ; \mathrm{D} ; \mathrm{E} ; \mathrm{F}$ & $\mathrm{A} ; \mathrm{B} ; \mathrm{E} ; \mathrm{F}$ & $\mathrm{A} ; \mathrm{B} ; \mathrm{E} ; \mathrm{F}$ & $\mathrm{A} ; \mathrm{B} ; \mathrm{C} ; \mathrm{D} ; \mathrm{F}$ & $\mathrm{A} ; \mathrm{B} ; \mathrm{C} ; \mathrm{D} ; \mathrm{E}$ \\
\hline Isophthalaldehyde & 58.32 & $\leq 1.00 \mathrm{E}-07$ & $\mathrm{D} ; \mathrm{E} ; \mathrm{F}$ & $\mathrm{D} ; \mathrm{E} ; \mathrm{F}$ & $\mathrm{D} ; \mathrm{E} ; \mathrm{F}$ & $\mathrm{A} ; \mathrm{B} ; \mathrm{C} ; \mathrm{E} ; \mathrm{F}$ & $\mathrm{A} ; \mathrm{B} ; \mathrm{C} ; \mathrm{D} ; \mathrm{F}$ & $\mathrm{A} ; \mathrm{B} ; \mathrm{C} ; \mathrm{D} ; \mathrm{E}$ \\
\hline Cyclotetradecane & 237.95 & $\leq 1.00 \mathrm{E}-07$ & $\mathrm{~B} ; \mathrm{C} ; \mathrm{D} ; \mathrm{E} ; \mathrm{F}$ & $\mathrm{A} ; \mathrm{C} ; \mathrm{D}$ & $\mathrm{A} ; \mathrm{B} ; \mathrm{D} ; \mathrm{E} ; \mathrm{F}$ & $\mathrm{A} ; \mathrm{B} ; \mathrm{C} ; \mathrm{E} ; \mathrm{F}$ & $\mathrm{A} ; \mathrm{C} ; \mathrm{D}$ & $\mathrm{A} ; \mathrm{C} ; \mathrm{D}$ \\
\hline Nonanoic Acid & 534.87 & $\leq 1.00 \mathrm{E}-07$ & $\mathrm{~B} ; \mathrm{C} ; \mathrm{E} ; \mathrm{F}$ & $\mathrm{A} ; \mathrm{C} ; \mathrm{D} ; \mathrm{F}$ & $\mathrm{A} ; \mathrm{B} ; \mathrm{D} ; \mathrm{E} ; \mathrm{F}$ & $\mathrm{B} ; \mathrm{C} ; \mathrm{E} ; \mathrm{F}$ & $\mathrm{A} ; \mathrm{C} ; \mathrm{D} ; \mathrm{F}$ & $\mathrm{A} ; \mathrm{B} ; \mathrm{C} ; \mathrm{D} ; \mathrm{E}$ \\
\hline 2-Ethyl-Phenol & 73.25 & $\leq 1.00 \mathrm{E}-07$ & $\mathrm{C} ; \mathrm{D} ; \mathrm{E} ; \mathrm{F}$ & $\mathrm{C} ; \mathrm{D} ; \mathrm{E}$ & $\mathrm{A} ; \mathrm{B} ; \mathrm{D} ; \mathrm{F}$ & $\mathrm{A} ; \mathrm{B} ; \mathrm{C} ; \mathrm{E} ; \mathrm{F}$ & $\mathrm{A} ; \mathrm{B} ; \mathrm{D} ; \mathrm{F}$ & $\mathrm{A} ; \mathrm{C} ; \mathrm{D} ; \mathrm{E}$ \\
\hline 2-Methoxy-4-Vinyl-Phenol & 531.37 & $\leq 1.00 \mathrm{E}-07$ & $\mathrm{~B} ; \mathrm{C} ; \mathrm{D} ; \mathrm{F}$ & $\mathrm{A} ; \mathrm{C} ; \mathrm{D} ; \mathrm{E} ; \mathrm{F}$ & $\mathrm{A} ; \mathrm{B} ; \mathrm{D} ; \mathrm{E} ; \mathrm{F}$ & $\mathrm{A} ; \mathrm{B} ; \mathrm{C} ; \mathrm{E} ; \mathrm{F}$ & $\mathrm{B} ; \mathrm{C} ; \mathrm{D} ; \mathrm{F}$ & $\mathrm{A} ; \mathrm{B} ; \mathrm{C} ; \mathrm{D} ; \mathrm{E}$ \\
\hline
\end{tabular}




\begin{tabular}{|c|c|c|c|c|c|c|c|c|}
\hline $\begin{array}{l}\text { 4-Methylimidazole } \\
\text { m-tert-Butyl-Toluene }\end{array}$ & $\begin{array}{c}543.79 \\
38.56\end{array}$ & $\begin{array}{l}\leq 1.00 \mathrm{E}-07 \\
\leq 1.00 \mathrm{E}-07\end{array}$ & $\begin{array}{l}\mathrm{B} ; \mathrm{C} ; \mathrm{D} ; \mathrm{E} ; \mathrm{F} \\
\quad \mathrm{D} ; \mathrm{E}\end{array}$ & $\begin{array}{l}\text { A; E; F } \\
\text { D; E }\end{array}$ & $\begin{array}{l}\mathrm{A} ; \mathrm{D} ; \mathrm{E} ; \mathrm{F} \\
\mathrm{D} ; \mathrm{E}\end{array}$ & $\begin{array}{c}\mathrm{A} ; \mathrm{C} ; \mathrm{E} ; \mathrm{F} \\
\mathrm{A} ; \mathrm{B} ; \mathrm{C} ; \mathrm{E} ; \mathrm{F}\end{array}$ & $\begin{array}{l}\mathrm{A} ; \mathrm{B} ; \mathrm{C} ; \mathrm{D} ; \mathrm{F} \\
\mathrm{A} ; \mathrm{B} ; \mathrm{C} ; \mathrm{D} ; \mathrm{F}\end{array}$ & $\begin{array}{c}\mathrm{A} ; \mathrm{B} ; \mathrm{C} ; \mathrm{D} ; \mathrm{E} \\
\mathrm{D} ; \mathrm{E}\end{array}$ \\
\hline Syringol & 72.89 & $\leq 1.00 \mathrm{E}-07$ & $\mathrm{~B} ; \mathrm{C} ; \mathrm{E} ; \mathrm{F}$ & $\mathrm{A} ; \mathrm{C} ; \mathrm{D} ; \mathrm{E} ; \mathrm{F}$ & $\mathrm{A} ; \mathrm{B} ; \mathrm{D}$ & $\mathrm{B} ; \mathrm{C} ; \mathrm{E} ; \mathrm{F}$ & $\mathrm{A} ; \mathrm{B} ; \mathrm{D}$ & $\mathrm{A} ; \mathrm{B} ; \mathrm{D}$ \\
\hline Decanoic Acid & 253.85 & $\leq 1.00 \mathrm{E}-07$ & $\mathrm{~B} ; \mathrm{E}$ & $\mathrm{A} ; \mathrm{C} ; \mathrm{D} ; \mathrm{E} ; \mathrm{F}$ & $\mathrm{B} ; \mathrm{E}$ & $\mathrm{B} ; \mathrm{E}$ & $\mathrm{A} ; \mathrm{B} ; \mathrm{C} ; \mathrm{D} ; \mathrm{F}$ & $\mathrm{B} ; \mathrm{E}$ \\
\hline -Hydroxy-2,3-Dihydro-Maltol & 492.74 & $\leq 1.00 \mathrm{E}-07$ & $\mathrm{~B} ; \mathrm{C} ; \mathrm{D} ; \mathrm{E} ; \mathrm{F}$ & $\mathrm{A} ; \mathrm{C} ; \mathrm{E} ; \mathrm{F}$ & $\mathrm{A} ; \mathrm{B} ; \mathrm{D} ; \mathrm{E} ; \mathrm{F}$ & $\mathrm{A} ; \mathrm{C} ; \mathrm{E} ; \mathrm{F}$ & $\mathrm{A} ; \mathrm{B} ; \mathrm{C} ; \mathrm{D}$ & $\mathrm{A} ; \mathrm{B} ; \mathrm{C} ; \mathrm{D}$ \\
\hline 5-Hydroxy-Maltol & 269.56 & $\leq 1.00 \mathrm{E}-07$ & $\mathrm{~B} ; \mathrm{E} ; \mathrm{F}$ & $\mathrm{A} ; \mathrm{C} ; \mathrm{D} ; \mathrm{E} ; \mathrm{F}$ & $\mathrm{B} ; \mathrm{E} ; \mathrm{F}$ & $\mathrm{B} ; \mathrm{E} ; \mathrm{F}$ & $\mathrm{A} ; \mathrm{B} ; \mathrm{C} ; \mathrm{D} ; \mathrm{F}$ & $\mathrm{A} ; \mathrm{B} ; \mathrm{C} ; \mathrm{D} ; \mathrm{E}$ \\
\hline Triethylene Glycol & 304.57 & $\leq 1.00 \mathrm{E}-07$ & $\mathrm{C} ; \mathrm{D} ; \mathrm{F}$ & $\mathrm{C} ; \mathrm{D} ; \mathrm{F}$ & $\mathrm{A} ; \mathrm{B} ; \mathrm{D} ; \mathrm{E}$ & $\mathrm{A} ; \mathrm{B} ; \mathrm{C} ; \mathrm{E} ; \mathrm{F}$ & $\mathrm{C} ; \mathrm{D} ; \mathrm{F}$ & $\mathrm{A} ; \mathrm{B} ; \mathrm{D} ; \mathrm{E}$ \\
\hline Benzoic Acid & 116.38 & $\leq 1.00 \mathrm{E}-07$ & $\mathrm{~B} ; \mathrm{C} ; \mathrm{E} ; \mathrm{F}$ & $\mathrm{A} ; \mathrm{D} ; \mathrm{E} ; \mathrm{F}$ & $\mathrm{A} ; \mathrm{D} ; \mathrm{E}$ & $\mathrm{B} ; \mathrm{C} ; \mathrm{E} ; \mathrm{F}$ & $\mathrm{A} ; \mathrm{B} ; \mathrm{C} ; \mathrm{D} ; \mathrm{F}$ & $\mathrm{A} ; \mathrm{B} ; \mathrm{D} ; \mathrm{E}$ \\
\hline 2-Methyl-Benzaldehyde & 255.45 & $\leq 1.00 \mathrm{E}-07$ & $\mathrm{D} ; \mathrm{E} ; \mathrm{F}$ & $\mathrm{C} ; \mathrm{D} ; \mathrm{E} ; \mathrm{F}$ & $\mathrm{B} ; \mathrm{D} ; \mathrm{E} ; \mathrm{F}$ & $\mathrm{A} ; \mathrm{B} ; \mathrm{C} ; \mathrm{F}$ & $\mathrm{A} ; \mathrm{B} ; \mathrm{C} ; \mathrm{F}$ & $\mathrm{A} ; \mathrm{B} ; \mathrm{C} ; \mathrm{D} ; \mathrm{E}$ \\
\hline Undecanoic Acid & 561.56 & $\leq 1.00 \mathrm{E}-07$ & $\mathrm{~B} ; \mathrm{D} ; \mathrm{E} ; \mathrm{F}$ & $\mathrm{A} ; \mathrm{C} ; \mathrm{D} ; \mathrm{F}$ & $\mathrm{B} ; \mathrm{E} ; \mathrm{F}$ & $\mathrm{A} ; \mathrm{B} ; \mathrm{E} ; \mathrm{F}$ & $\mathrm{A} ; \mathrm{C} ; \mathrm{D} ; \mathrm{F}$ & $\mathrm{A} ; \mathrm{B} ; \mathrm{C} ; \mathrm{D} ; \mathrm{E}$ \\
\hline 4-Pyridinol & 160.11 & $\leq 1.00 \mathrm{E}-07$ & $\mathrm{~B} ; \mathrm{C} ; \mathrm{E} ; \mathrm{F}$ & $\mathrm{A} ; \mathrm{C} ; \mathrm{D} ; \mathrm{F}$ & $\mathrm{A} ; \mathrm{B} ; \mathrm{D} ; \mathrm{E} ; \mathrm{F}$ & $\mathrm{B} ; \mathrm{C} ; \mathrm{E} ; \mathrm{F}$ & $\mathrm{A} ; \mathrm{C} ; \mathrm{D} ; \mathrm{F}$ & $\mathrm{A} ; \mathrm{B} ; \mathrm{C} ; \mathrm{D} ; \mathrm{E}$ \\
\hline 4-Amino-Phenol & 886.23 & $\leq 1.00 \mathrm{E}-07$ & $\mathrm{~B} ; \mathrm{C} ; \mathrm{D} ; \mathrm{E} ; \mathrm{F}$ & $\mathrm{A} ; \mathrm{C} ; \mathrm{D} ; \mathrm{E}$ & $\mathrm{A} ; \mathrm{B} ; \mathrm{D} ; \mathrm{E}$ & $\mathrm{A} ; \mathrm{B} ; \mathrm{C} ; \mathrm{E} ; \mathrm{F}$ & $\mathrm{A} ; \mathrm{B} ; \mathrm{C} ; \mathrm{D} ; \mathrm{F}$ & $\mathrm{A} ; \mathrm{D} ; \mathrm{E}$ \\
\hline
\end{tabular}

${ }^{1} \mathrm{~F}$ Value - Fischer value from One-way ANOVA test.

${ }^{2} \mathrm{P}$ Value - Probability value from One-way ANOVA test.

${ }^{3}$ Samples with significantly different relative peak area values determined by one-way ANOVA with Post-hoc Tukey test $(\alpha<0.05)$.

Table S8. Information summary of Principal Component Analysis (PCA) and Partial Least Square Analysis (PLS) based on the relative peak areas of the identified VOCs in sugarcane samples.

\begin{tabular}{cccccccc}
\hline Analysis & Component & $\mathbf{R}^{\mathbf{2}} \mathbf{X}$ & Eigenvalues & $\mathbf{R}^{\mathbf{2}} \mathbf{Y}$ & $\mathbf{Q}^{\mathbf{2}}$ & Significance & Iterations \\
\hline \multirow{6}{*}{ PCA } & 1 & 0.320 & 86.86 & n.a. & 0.266 & $\mathrm{~S}$ & 16 \\
& 2 & 0.219 & 59.46 & n.a. & 0.183 & $\mathrm{~S}$ & 9 \\
& 3 & 0.159 & 43.10 & n.a. & 0.279 & $\mathrm{~S}$ & 6 \\
& 4 & 0.113 & 30.70 & n.a. & 0.315 & $\mathrm{~S}$ & 10 \\
& 5 & 0.085 & 23.11 & n.a. & 0.418 & $\mathrm{~S}$ & 5 \\
& 6 & 0.024 & 6.63 & n.a. & 0.009 & $\mathrm{~S}$ & 31
\end{tabular}




\begin{tabular}{|c|c|c|c|c|c|c|c|}
\hline & 7 & 0.020 & 5.40 & n.a. & 0.152 & $\mathrm{~S}$ & 5 \\
\hline & 8 & 0.015 & 3.99 & n.a. & 0.096 & $\mathrm{~S}$ & 26 \\
\hline & 9 & 0.013 & 3.40 & n.a. & 0.011 & $\mathrm{~S}$ & 29 \\
\hline & 10 & 0.012 & 3.16 & n.a. & 0.180 & $\mathrm{~S}$ & 23 \\
\hline & 11 & 0.010 & 2.67 & n.a. & 0.469 & $\mathrm{~S}$ & 4 \\
\hline & 12 & 0.003 & 0.81 & n.a. & 0.240 & $\mathrm{~S}$ & 6 \\
\hline & 13 & 0.001 & 0.29 & n.a. & 0.045 & $\mathrm{~S}$ & 7 \\
\hline & 14 & 0.001 & 0.21 & n.a. & -0.053 & UNKNOWN & 17 \\
\hline \multirow{18}{*}{ PLS } & 1 & 0.328 & 86.95 & 0.091 & 0.014 & $\mathrm{~S}$ & 14 \\
\hline & 2 & 0.225 & 59.56 & 0.091 & 0.029 & $\mathrm{~S}$ & 8 \\
\hline & 3 & 0.163 & 43.19 & 0.090 & 0.042 & $\mathrm{~S}$ & 9 \\
\hline & 4 & 0.116 & 30.80 & 0.091 & 0.059 & $\mathrm{~S}$ & 10 \\
\hline & 5 & 0.088 & 23.21 & 0.091 & 0.073 & $\mathrm{~S}$ & 4 \\
\hline & 6 & 0.021 & 5.57 & 0.090 & 0.051 & $\mathrm{~S}$ & 8 \\
\hline & 7 & 0.016 & 4.31 & 0.091 & 0.089 & $\mathrm{~S}$ & 6 \\
\hline & 8 & 0.011 & 2.92 & 0.087 & 0.107 & $\mathrm{~S}$ & 10 \\
\hline & 9 & 0.009 & 2.32 & 0.090 & 0.168 & $\mathrm{~S}$ & 16 \\
\hline & 10 & 0.008 & 2.08 & 0.088 & 0.331 & $\mathrm{~S}$ & 21 \\
\hline & 11 & 0.006 & 1.60 & 0.089 & 0.851 & $\mathrm{~S}$ & 4 \\
\hline & 12 & 0.003 & 0.70 & 0.008 & 0.607 & $\mathrm{~S}$ & 4 \\
\hline & 13 & 0.001 & 0.21 & 0.002 & 0.176 & $\mathrm{~S}$ & 7 \\
\hline & 14 & 0.001 & 0.17 & 0.001 & -0.072 & $\mathrm{~S}$ & 50 \\
\hline & 15 & 0.001 & 0.18 & 0.001 & 0.036 & $\mathrm{~S}$ & 13 \\
\hline & 16 & 0.001 & 0.18 & 0.000 & 0.072 & $\mathrm{~S}$ & 50 \\
\hline & 17 & 0.001 & 0.12 & 0.000 & 0.100 & $\mathrm{~S}$ & 14 \\
\hline & 18 & 0.000 & 0.14 & 0.000 & 0.152 & $\mathrm{~S}$ & 13 \\
\hline
\end{tabular}


Table S9. Loading results and variable importance in projection scores of variables from Principal Component Analysis (PCA) and Partial Least Squares Analysis (PLS) based on the relative peak areas of the identified VOCs in sugarcane samples.

\begin{tabular}{|c|c|c|c|c|c|c|c|c|c|c|c|c|}
\hline \multirow{3}{*}{$\begin{array}{c}\text { ID } \\
\text { Number }\end{array}$} & \multirow{3}{*}{ Volatile Organic Compound } & \multirow{3}{*}{ Abbreviation } & \multicolumn{5}{|c|}{ PCA } & \multicolumn{5}{|c|}{ PLS } \\
\hline & & & \multirow[b]{2}{*}{1} & \multirow[b]{2}{*}{2} & \multirow[b]{2}{*}{3} & \multicolumn{2}{|c|}{ VIP' } & \multirow[b]{2}{*}{1} & \multirow[b]{2}{*}{2} & \multirow[b]{2}{*}{3} & \multicolumn{2}{|c|}{ VIP' } \\
\hline & & & & & & Importance & $\begin{array}{l}\text { Power } \\
\text { (x 100) }\end{array}$ & & & & Importance & $\begin{array}{l}\text { Power } \\
\left(\begin{array}{ll}x & 100)\end{array}\right)\end{array}$ \\
\hline 1 & Pentane & PTANE & 0.198 & 0.827 & -0.155 & 185 & 99.52 & 0.019 & 0.107 & 0.024 & 64 & 6.42 \\
\hline 2 & 1-Pentene & PT1ENE & -0.591 & 0.507 & 0.264 & 219 & 99.20 & -0.065 & 0.064 & -0.040 & 46 & 7.13 \\
\hline 3 & 2-Methyl-Pentane & M2PTANE & 0.364 & 0.806 & 0.320 & 42 & 99.93 & 0.037 & 0.106 & -0.047 & 127 & 5.35 \\
\hline 4 & Hexane & HEXANE & -0.935 & -0.006 & 0.063 & 30 & 99.94 & -0.100 & -0.003 & -0.009 & 91 & 5.98 \\
\hline 5 & Ethyl Ether & EETHR & -0.482 & -0.275 & 0.022 & 195 & 99.47 & -0.051 & -0.037 & -0.005 & 2 & 13.71 \\
\hline 6 & cis-4-Methyl-2-Pentene & CM4PT2ENE & 0.205 & 0.638 & 0.273 & 176 & 99.60 & 0.020 & 0.084 & -0.040 & 11 & 9.87 \\
\hline 7 & cis-Piperylene & CPIPERLNE & 0.156 & 0.767 & 0.303 & 94 & 99.82 & 0.015 & 0.100 & -0.044 & 31 & 7.88 \\
\hline 8 & 2-Methyl-1-Pentene & M2PT1ENE & -0.556 & -0.223 & -0.041 & 92 & 99.83 & -0.059 & -0.030 & 0.006 & 7 & 11.84 \\
\hline 9 & cis-3-Methyl-2-Pentene & CM3PT2ENE & 0.183 & -0.250 & -0.287 & 122 & 99.77 & 0.020 & -0.033 & 0.042 & 13 & 9.66 \\
\hline 10 & 3-Methyl-Hexane & M3HXANE & -0.921 & 0.010 & -0.259 & 178 & 99.59 & -0.099 & -0.002 & 0.040 & 149 & 5.20 \\
\hline 11 & 1,4-Pentadiene & PT14DIENE & 0.098 & 0.507 & 0.240 & 111 & 99.80 & 0.009 & 0.066 & -0.037 & 10 & 10.63 \\
\hline 12 & Methanethiol & METHIOL & 0.026 & 0.782 & 0.312 & 149 & 99.69 & 0.001 & 0.102 & -0.047 & 54 & 6.67 \\
\hline 13 & trans-Piperylene & TPIPERLNE & -0.004 & 0.487 & 0.493 & 184 & 99.53 & -0.002 & 0.064 & -0.076 & 17 & 8.91 \\
\hline 14 & Heptane & HPTANE & -0.574 & 0.406 & 0.072 & 188 & 99.51 & -0.063 & 0.051 & -0.012 & 56 & 6.64 \\
\hline 15 & tert-Butyl Ethyl Ether & BEETHR & -0.894 & -0.214 & -0.015 & 123 & 99.77 & -0.095 & -0.030 & 0.002 & 61 & 6.47 \\
\hline 16 & Ethanal & ETAL & -0.307 & -0.300 & 0.820 & 48 & 99.92 & -0.032 & -0.038 & -0.125 & 41 & 7.26 \\
\hline 17 & Dimethyl Sulfide & DMSULFI & 0.647 & -0.564 & 0.224 & 248 & 98.87 & 0.071 & -0.071 & -0.035 & 52 & 6.80 \\
\hline 18 & 4-Methyl-Heptane & M4HPTANE & -0.844 & -0.197 & 0.048 & 17 & 99.96 & -0.090 & -0.028 & -0.007 & 28 & 7.99 \\
\hline 19 & Octane & OCTANE & -0.597 & 0.379 & 0.218 & 155 & 99.67 & -0.065 & 0.048 & -0.034 & 119 & 5.41 \\
\hline 20 & Propanal & PPAL & -0.903 & -0.103 & 0.261 & 225 & 99.15 & -0.096 & -0.015 & -0.040 & 139 & 5.26 \\
\hline 21 & Furan & FUR & 0.449 & 0.057 & 0.616 & 241 & 98.98 & 0.048 & 0.010 & -0.095 & 15 & 9.23 \\
\hline 22 & 2,4-Dimethyl-Heptane & DM24HPTANE & 0.492 & 0.688 & 0.204 & 259 & 98.59 & 0.051 & 0.091 & -0.029 & 19 & 8.78 \\
\hline
\end{tabular}


2-Methyl-Propanal

2-Propanone

Methyl Acetate

2-Methyl-2-Heptene Crotonal

2,3-Dimethyl-Heptane

4-Methyl-Octane

2-Methyl-Furan

2,4-Dimethyl-1-Heptene

Ethyl Acetate

Nonane

2-Butanone

2-Methyl-Butanal

3-Methyl-Butanal

Ethyl Alcohol

Benzene

3-Ethyl-Heptane

2-Ethyl-Furan

Ethyl Propanoate

Ethyl 2-Methylpropanoate

2-Methyl-Crotonal

Propyl Acetate

3-Pentanone

Pentanal

2,3-Butanedione

2,3,4-Trimethyl-Hexane Decane

2,6-Dimethyl-Nonane

2,5-Dimethyl-Nonane

4-Methyl-2-Pentanone

$\begin{array}{ccccc}\text { MPPAL } & 0.231 & 0.386 & 0.375 & 77 \\ \text { PP2ONE } & -0.650 & -0.550 & 0.161 & 116 \\ \text { MESTAA } & 0.182 & 0.651 & 0.290 & 7 \\ \text { M2HPTENE } & -0.967 & -0.095 & -0.015 & 1 \\ \text { CROTNAL } & 0.270 & 0.469 & -0.462 & 159 \\ \text { DM23HPTANE } & 0.426 & 0.584 & 0.447 & 90 \\ \text { M4OCTANE } & 0.306 & 0.890 & 0.217 & 192 \\ \text { M2FUR } & 0.273 & -0.389 & -0.347 & 240 \\ \text { DM24HPT1ENE } & -0.977 & -0.116 & -0.016 & 4 \\ \text { EESTAA } & 0.012 & 0.951 & 0.274 & 32 \\ \text { NNANE } & -0.632 & 0.556 & -0.231 & 180 \\ \text { BT2ONE } & -0.867 & 0.042 & 0.351 & 209 \\ \text { M2BTAL } & -0.013 & -0.341 & 0.861 & 137 \\ \text { M3BTAL } & -0.166 & -0.395 & 0.840 & 210 \\ \text { ETOL } & 0.747 & 0.298 & -0.547 & 224 \\ \text { BNZ } & 0.169 & 0.672 & -0.089 & 234 \\ \text { E3HPTANE } & -0.912 & 0.063 & 0.143 & 170 \\ \text { E2FUR } & 0.152 & 0.314 & -0.082 & 257 \\ \text { EESTPA } & 0.081 & 0.937 & 0.169 & 11 \\ \text { M2EESTPA } & 0.056 & 0.949 & 0.199 & 27 \\ \text { M2CROTNAL } & 0.301 & 0.541 & 0.329 & 95 \\ \text { PESTAA } & 0.081 & 0.931 & 0.229 & 3 \\ \text { PT3ONE } & -0.769 & -0.013 & 0.191 & 242 \\ \text { PTNAL } & -0.991 & 0.031 & 0.036 & 26 \\ \text { BT23DONE } & 0.693 & 0.388 & -0.066 & 247 \\ \text { TM234HXANE } & -0.979 & -0.091 & -0.013 & 28 \\ \text { DCANE } & -0.935 & -0.022 & -0.114 & 167 \\ \text { DM26NNANE } & -0.955 & -0.081 & 0.027 & 68 \\ \text { DM25NNANE } & 0.188 & 0.974 & 0.066 & 29 \\ \text { M4PT2ONE } & -0.967 & -0.170 & 0.025 & 10\end{array}$

99.86

$\begin{array}{lllll}99.97 & 0.018 & 0.085 & -0.043\end{array}$

4.63

$\begin{array}{lllll}99.99 & -0.103 & -0.015 & 0.002\end{array}$

$\begin{array}{llll}99.66 & 0.028 & 0.061 & 0.073\end{array}$

$\begin{array}{lllll}99.83 & 0.044 & 0.078 & -0.067\end{array}$

$\begin{array}{lllll}99.48 & 0.030 & 0.117 & -0.031\end{array}$

$\begin{array}{lllll}99.01 & 0.030 & -0.051 & 0.050\end{array}$

$\begin{array}{llll}99.98 & -0.104 & -0.018 & 0.002\end{array}$

$\begin{array}{lllll}99.94 & -0.001 & 0.124 & -0.040\end{array}$

$\begin{array}{lllll}99.57 & -0.069 & 0.070 & 0.036\end{array}$

$\begin{array}{lllll}99.34 & -0.093 & 0.004 & -0.052\end{array}$

$\begin{array}{llll}99.74 & -0.001 & -0.042 & -0.132\end{array}$

$\begin{array}{lllll}99.34 & -0.017 & -0.050 & -0.129\end{array}$

$\begin{array}{lllll}99.15 & 0.079 & 0.039 & 0.084\end{array}$

$\begin{array}{llll}99.06 & 0.016 & 0.088 & 0.015\end{array}$

$\begin{array}{llll}99.63 & -0.098 & 0.006 & -0.022\end{array}$

$\begin{array}{lllll}98.61 & 0.015 & 0.041 & 0.014\end{array}$

$\begin{array}{lllll}99.96 & 0.006 & 0.122 & -0.024\end{array}$

$\begin{array}{lllll}99.94 & 0.003 & 0.123 & -0.029\end{array}$

$\begin{array}{lllll}99.82 & 0.031 & 0.072 & -0.047\end{array}$

$\begin{array}{lllll}99.98 & 0.006 & 0.121 & -0.033\end{array}$

$\begin{array}{lllll}98.97 & -0.082 & -0.003 & -0.029\end{array}$

$\begin{array}{lllll}99.95 & -0.106 & 0.001 & -0.005\end{array}$

$\begin{array}{lllll}98.88 & 0.073 & 0.052 & 0.011\end{array}$

$\begin{array}{lllll}99.94 & -0.105 & -0.014 & 0.002\end{array}$

$\begin{array}{lllll}99.63 & -0.100 & -0.006 & 0.018\end{array}$

$\begin{array}{lllll}99.88 & -0.102 & -0.013 & -0.004\end{array}$

$\begin{array}{lllll}99.94 & 0.017 & 0.127 & -0.008\end{array}$

$\begin{array}{lllll}99.97 & -0.103 & -0.024 & -0.004\end{array}$
10.64

4.63

9.83

7.81

5.22

8.19

4.09

4.26

7.97

4.52

6.42

7.11

4.56

8.24

5.00

13.06

5.25

4.86

5.84

5.27

5.77

3.70

5.21

4.07

5.21

5.07

4.05 


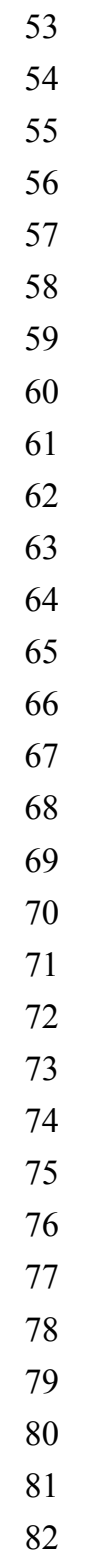

2-Methy-2-Butanol
2-Methylpropyl Acetate
a-Pinene
4,4-Dimethyl-2-Pentanone
1-Penten-3-one
5-Butyl-Nonane
3,6-Dimethyl-Decane
2-Methyl-3-Buten-2-ol
5-Methyl-Decane
4-Methyl-Decane
2,3-Pentanedione

Methyl 3,3-Dimethylbutanoate

$$
\text { 2-Hexanone }
$$

4-Methyl-Undecane Hexanal

2-Methyl-1-Propanol

2-Methyl-2-Pentanol

Ethyl-Benzene

3-Methylbutyl Acetate

$$
\text { o-Xylene }
$$

2-Pentanol

$$
\text { p-Xylene }
$$

5-Methyl-2-Hexanone

Methyl 2-Ethylpentanoate

$$
\text { 1-Butanol }
$$

$\alpha$-Phellandrene

2-Methyl-3-Pentanol

1-Penten-3-ol

$\alpha$-Terpinene

Butyl Prop-2-enoate

$\begin{array}{ccccc}\text { M2BT2OL } & -0.976 & -0.118 & -0.003 & 6 \\ \text { M2PESTAA } & 0.145 & 0.873 & 0.321 & 117 \\ \text { APNENE } & -0.551 & 0.772 & -0.047 & 133 \\ \text { DM44PT2ONE } & -0.986 & -0.116 & 0.004 & 50 \\ \text { PT1E3ONE } & -0.925 & 0.302 & 0.104 & 144 \\ \text { B5NONANE } & 0.237 & 0.832 & 0.246 & 2 \\ \text { DM36DCANE } & 0.429 & 0.297 & -0.219 & 135 \\ \text { M3BT2OL } & 0.070 & 0.922 & 0.088 & 86 \\ \text { M5DCANE } & 0.813 & 0.117 & 0.173 & 140 \\ \text { M4DCANE } & 0.031 & 0.486 & 0.357 & 253 \\ \text { PTDONE } & 0.292 & -0.372 & 0.813 & 98 \\ \text { DM33MESTBA } & 0.099 & 0.908 & 0.159 & 35 \\ \text { HX2ONE } & -0.059 & 0.828 & -0.098 & 161 \\ \text { M4UNCANE } & 0.154 & 0.926 & -0.023 & 88 \\ \text { HXAL } & -0.834 & 0.351 & 0.287 & 82 \\ \text { M2PP1OL } & 0.619 & -0.214 & -0.349 & 191 \\ \text { M2PT2OL } & -0.906 & -0.062 & 0.273 & 124 \\ \text { ETBNZ } & 0.266 & 0.833 & 0.189 & 45 \\ \text { M3BESTAA } & 0.094 & 0.468 & 0.183 & 264 \\ \text { OXYLNE } & -0.958 & 0.095 & 0.115 & 25 \\ \text { PT2OL } & 0.067 & 0.957 & 0.148 & 5 \\ \text { PXYLNE } & -0.321 & 0.701 & -0.080 & 119 \\ \text { M5HX2AONE } & -0.347 & 0.855 & 0.248 & 84 \\ \text { E2MESTPTA } & 0.018 & 0.945 & 0.179 & 55 \\ \text { BT1OL } & 0.236 & 0.728 & -0.449 & 179 \\ \text { APHELDNE } & -0.928 & -0.154 & 0.011 & 37 \\ \text { M2PT3OL } & 0.160 & 0.915 & 0.351 & 126 \\ \text { PT1E3OL } & -0.415 & 0.626 & -0.264 & 168 \\ \text { ATERPNNE } & -0.948 & -0.186 & 0.064 & 31 \\ \text { BTESTPPEA } & -0.552 & 0.632 & 0.171 & 76\end{array}$

$\begin{array}{lccccc}99.98 & -0.104 & -0.018 & 0.000 & 246 & 4.01 \\ 99.79 & 0.013 & 0.114 & -0.047 & 73 & 6.28 \\ 99.75 & -0.061 & 0.098 & 0.009 & 111 & 5.51 \\ 99.92 & -0.105 & -0.018 & -0.001 & 264 & 3.56 \\ 99.70 & -0.100 & 0.037 & -0.015 & 253 & 3.88 \\ 99.98 & 0.023 & 0.109 & -0.037 & 221 & 4.46 \\ 99.75 & 0.045 & 0.039 & 0.031 & 90 & 5.98 \\ 99.84 & 0.005 & 0.120 & -0.012 & 93 & 5.85 \\ 99.74 & 0.087 & 0.018 & -0.026 & 43 & 7.24 \\ 98.78 & 0.002 & 0.063 & -0.054 & 18 & 8.83 \\ 99.82 & 0.032 & -0.046 & -0.124 & 99 & 5.76 \\ 99.94 & 0.008 & 0.118 & -0.023 & 80 & 6.16 \\ 99.66 & -0.008 & 0.107 & 0.016 & 21 & 8.45 \\ 99.84 & 0.014 & 0.120 & 0.006 & 87 & 6.03 \\ 99.85 & -0.090 & 0.044 & -0.043 & 198 & 4.72 \\ 99.50 & 0.067 & -0.027 & 0.053 & 100 & 5.75 \\ 99.77 & -0.097 & -0.010 & -0.042 & 196 & 4.73 \\ 99.92 & 0.026 & 0.109 & -0.028 & 36 & 7.66 \\ 98.14 & 0.009 & 0.061 & -0.029 & 72 & 6.29 \\ 99.95 & -0.103 & 0.010 & -0.017 & 208 & 4.61 \\ 99.98 & 0.005 & 0.124 & -0.021 & 172 & 5.01 \\ 99.78 & -0.036 & 0.090 & 0.015 & 82 & 6.12 \\ 99.85 & -0.040 & 0.110 & -0.037 & 202 & 4.65 \\ 99.91 & -0.001 & 0.123 & -0.026 & 143 & 5.22 \\ 99.58 & 0.023 & 0.094 & 0.070 & 101 & 5.74 \\ 99.94 & -0.099 & -0.022 & -0.002 & 95 & 5.82 \\ 99.76 & 0.015 & 0.120 & -0.051 & 238 & 4.20 \\ 99.63 & -0.046 & 0.079 & 0.040 & 44 & 7.22 \\ 99.94 & -0.101 & -0.026 & -0.010 & 235 & 4.23 \\ 99.87 & -0.061 & 0.081 & -0.024 & 33 & 7.86\end{array}$




$\begin{array}{lc}83 & \text { m-Xylene } \\ 84 & \text { 2-Heptanone } \\ 85 & \text { Heptanal } \\ 86 & \text { D-Limonene } \\ 87 & \text { 3-Hexanol } \\ 88 & \text { 3-Phellandrene } \\ 89 & \text { Eucalyptol } \\ 90 & \text { m-Menthane } \\ 91 & \text { 4-Methyl-2-Heptanone } \\ 92 & \text { 3-Methyl-2-Pentanol } \\ 93 & \text { 2-Methyl-1-Butanol } \\ 94 & \text { 3-Methyl-1-Butanol } \\ 95 & \text { o-Ethyltoluene } \\ 96 & \text { m-Ethyltoluene } \\ 97 & \text { 2-Hexanol } \\ 98 & \text { Ethylene Glycol Ethyl Ether } \\ 99 & \text { 6-Methyl-2-Heptanone } \\ 100 & \text { Pseudocumene } \\ 101 & \text { 2-Octanone } \\ 102 & \text { 4,6-Dimethyl-2-Heptanone } \\ 103 & \text { 2,3-Dimethyl-2-Hexanol } \\ 104 & \text { 3-Octanone } \\ 105 & \text { Styrene } \\ 106 & \text { 4-Methyl-2-Heptanone } \\ 107 & \text { Mesitylene } \\ 108 & \text {-Methyl-4-Heptanol } \\ 109 & \\ 110 & \\ 111 & \end{array}$

\begin{tabular}{|c|c|c|c|c|}
\hline MXYLNE & -0.513 & 0.753 & -0.225 & 129 \\
\hline HPT2ONE & 0.101 & -0.345 & -0.812 & 181 \\
\hline HPTAL & -0.117 & 0.310 & -0.311 & 99 \\
\hline DLIMNENE & -0.349 & 0.856 & -0.170 & 163 \\
\hline HX3OL & 0.372 & -0.222 & -0.207 & 139 \\
\hline BPHELDNE & -0.885 & -0.143 & -0.014 & 78 \\
\hline EUCPTOL & -0.826 & -0.260 & 0.247 & 120 \\
\hline MMTHANE & -0.751 & 0.592 & 0.033 & 21 \\
\hline M4HPT2ONE & -0.976 & -0.163 & -0.023 & 14 \\
\hline M2PT2OL & 0.445 & 0.478 & -0.666 & 156 \\
\hline M2BT1OL & 0.797 & 0.184 & 0.003 & 142 \\
\hline M3BT1OL & 0.814 & 0.248 & 0.178 & 118 \\
\hline OETOLENE & -0.385 & 0.708 & 0.094 & 172 \\
\hline METOLENE & 0.261 & 0.896 & 0.023 & 24 \\
\hline $\mathrm{HX} 2 \mathrm{OL}$ & 0.425 & -0.510 & 0.213 & 100 \\
\hline ENGOLEETHR & 0.187 & -0.012 & 0.272 & 38 \\
\hline M6HPT2ONE & -0.985 & -0.018 & 0.072 & 151 \\
\hline PSEUDCUMNE & -0.194 & 0.787 & 0.169 & 201 \\
\hline DM46HPT2ONE & -0.964 & -0.141 & 0.004 & 20 \\
\hline DM23HX2OL & -0.991 & -0.091 & 0.013 & 8 \\
\hline OCT3ONE & 0.452 & 0.526 & -0.567 & 49 \\
\hline STYNE & 0.298 & 0.878 & 0.126 & 198 \\
\hline M5HPTAONE & -0.805 & -0.118 & -0.180 & 148 \\
\hline PT1OL & 0.545 & -0.574 & 0.502 & 13 \\
\hline PETOLENE & 0.148 & 0.872 & -0.073 & 96 \\
\hline PCYMNE & -0.905 & -0.158 & 0.095 & 64 \\
\hline MESTLNE & -0.025 & -0.538 & -0.106 & 60 \\
\hline M4HPT4OL & -0.173 & -0.621 & 0.448 & 81 \\
\hline HPT4OL & -0.933 & -0.036 & -0.001 & 61 \\
\hline OCT2ONE & -0.250 & -0.433 & -0.732 & 207 \\
\hline
\end{tabular}

$\begin{array}{llll}99.76 & -0.057 & 0.096 & 0.035 \\ 99.57 & 0.012 & -0.046 & 0.122 \\ 99.81 & -0.013 & 0.039 & 0.046 \\ 99.66 & -0.040 & 0.110 & 0.028 \\ 99.74 & 0.041 & -0.028 & 0.033 \\ 99.86 & -0.095 & -0.021 & 0.002 \\ 99.78 & -0.088 & -0.035 & -0.037 \\ 99.95 & -0.082 & 0.075 & -0.004 \\ 99.96 & -0.104 & -0.024 & 0.003 \\ 99.67 & 0.047 & 0.061 & 0.101 \\ 99.73 & 0.085 & 0.026 & -0.001 \\ 99.79 & 0.086 & 0.035 & -0.027 \\ 99.62 & -0.043 & 0.091 & -0.013 \\ 99.95 & 0.026 & 0.117 & -0.001 \\ 99.81 & 0.047 & -0.065 & -0.036 \\ 99.94 & 0.020 & 0.000 & -0.039 \\ 99.69 & -0.106 & -0.005 & -0.011 \\ 99.42 & -0.023 & 0.102 & -0.024 \\ 99.95 & -0.103 & -0.021 & -0.001 \\ 99.97 & -0.106 & -0.014 & -0.002 \\ 99.92 & 0.047 & 0.068 & 0.086 \\ 99.44 & 0.030 & 0.115 & -0.017 \\ 99.69 & -0.086 & -0.018 & 0.027 \\ 99.96 & 0.060 & -0.072 & -0.078 \\ 99.82 & 0.013 & 0.113 & 0.014 \\ 99.90 & -0.097 & -0.023 & -0.014 \\ 99.90 & -0.001 & -0.070 & 0.017 \\ 99.85 & -0.017 & -0.080 & -0.071 \\ 99.90 & -0.100 & -0.007 & 0.001 \\ 99.37 & -0.026 & -0.059 & 0.109 \\ & & & \end{array}$

$\begin{array}{cc}148 & 5.20 \\ 130 & 5.34 \\ 60 & 6.49 \\ 195 & 4.73 \\ 105 & 5.64 \\ 47 & 7.12 \\ 108 & 5.60 \\ 158 & 5.14 \\ 261 & 3.69 \\ 184 & 4.91 \\ 66 & 6.40 \\ 51 & 6.89 \\ 55 & 6.67 \\ 215 & 4.54 \\ 156 & 5.15 \\ 35 & 7.79 \\ 262 & 3.65 \\ 38 & 7.59 \\ 220 & 4.50 \\ 265 & 3.54 \\ 114 & 5.44 \\ 112 & 5.48 \\ 39 & 7.54 \\ 107 & 5.61 \\ 188 & 4.87 \\ 201 & 4.68 \\ 57 & 6.51 \\ 20 & 8.65 \\ 123 & 5.38 \\ 131 & 5.34\end{array}$




\begin{tabular}{|c|c|}
\hline 113 & Octanal \\
\hline 114 & 3-Hydroxy-2-Butanone \\
\hline 115 & 1-Octen-3-one \\
\hline 116 & trans-2-Pentenol \\
\hline 117 & 2,2,6-Trimethyl-Cyclohexanone \\
\hline 118 & 2-Heptanol \\
\hline 119 & cis-2-Pentenol \\
\hline 120 & 1-Hydroxy-2-Propanone \\
\hline 121 & 5-Methyl-4-Hexen-3-one \\
\hline 122 & Hemimellitene \\
\hline 123 & 6-Methyl-5-Hepten-2-one \\
\hline 124 & 2-Pentene \\
\hline 125 & 1-Hexanol \\
\hline 126 & 2-Cyclopenten-1-one \\
\hline 127 & 2-Methyl-2-Cyclopenten-1-one \\
\hline 128 & $\beta$-Methy-Styrene \\
\hline 129 & 4-Octanol \\
\hline 130 & cis-3-Hexen-1-ol \\
\hline 131 & 2-Nonanone \\
\hline 132 & Nonanal \\
\hline 133 & Ethylene Glycol Butyl Ether \\
\hline 134 & 1-Ethyl-1-Methyl-Cyclopentane \\
\hline 135 & 3-Octen-2-one \\
\hline 136 & 2-Octanol \\
\hline 137 & 3-Ethyl-2-Methyl-1,3-Hexadiene \\
\hline 138 & 1,3-Bis(1,1-Dimethylethyl)-Benzene \\
\hline 139 & trans-2-Octenal \\
\hline 140 & m-Ethyl-Styrene \\
\hline 141 & 5-Methyl-2-Heptanone \\
\hline 142 & 2-Decanone \\
\hline
\end{tabular}

$\begin{array}{ccccc}\text { OCTAL } & -0.682 & 0.661 & 0.050 & 91 \\ \text { HXY3BT2ONE } & 0.577 & -0.444 & -0.309 & 214 \\ \text { OCT3E1ONE } & -0.532 & -0.472 & 0.493 & 36 \\ \text { TPT1E2OL } & 0.214 & 0.568 & -0.309 & 243 \\ \text { TM226CHXONE } & -0.362 & 0.843 & -0.109 & 256 \\ \text { HPT2OL } & 0.417 & -0.680 & -0.334 & 252 \\ \text { CPT1E2OL } & 0.379 & -0.363 & 0.751 & 229 \\ \text { HXY1PP2ONE } & 0.300 & -0.218 & 0.447 & 12 \\ \text { M5HX3E4ONE } & 0.253 & -0.106 & -0.770 & 230 \\ \text { HEMIMLTNE } & 0.218 & 0.801 & 0.372 & 211 \\ \text { M6HPT2E5ONE } & -0.918 & 0.072 & 0.215 & 72 \\ \text { PT2ENE } & -0.005 & -0.723 & -0.564 & 173 \\ \text { HX1OL } & 0.738 & 0.326 & 0.528 & 132 \\ \text { CY2PT1EONE } & -0.288 & 0.261 & 0.194 & 222 \\ \text { M2CY2PT1EONE } & -0.444 & 0.120 & -0.373 & 101 \\ \text { BMSTYNE } & 0.364 & 0.692 & -0.160 & 200 \\ \text { OCT4OL } & -0.676 & -0.635 & -0.174 & 227 \\ \text { CHX1E3OL } & 0.634 & 0.125 & 0.502 & 223 \\ \text { NON2ONE } & 0.437 & -0.123 & -0.678 & 125 \\ \text { NONAL } & 0.715 & -0.530 & 0.081 & 85 \\ \text { ENGOLBETHR } & 0.486 & -0.137 & 0.778 & 146 \\ \text { E1M1CYPTANE } & -0.987 & 0.042 & 0.034 & 41 \\ \text { OCT2E3ONE } & 0.025 & -0.212 & -0.312 & 22 \\ \text { OCT2OL } & 0.394 & -0.396 & -0.770 & 114 \\ \text { E3M2HX13DENE } & 0.258 & 0.278 & -0.685 & 154 \\ \text { BI13DME11BNZ } & -0.968 & -0.154 & -0.007 & 109 \\ \text { TOCT2EAL } & -0.875 & 0.372 & -0.192 & 52 \\ \text { MESTYNE } & -0.680 & -0.447 & 0.037 & 136 \\ \text { M5HPT2ONE } & -0.969 & -0.140 & 0.016 & 73 \\ \text { DEC2ONE } & -0.844 & -0.199 & -0.311 & 215\end{array}$

$\begin{array}{lccccc}99.83 & -0.075 & 0.084 & -0.005 & 240 & 4.14 \\ 99.29 & 0.063 & -0.057 & 0.045 & 22 & 8.41 \\ 99.94 & -0.056 & -0.062 & -0.077 & 89 & 5.99 \\ 98.97 & 0.021 & 0.073 & 0.048 & 14 & 9.33 \\ 98.67 & -0.041 & 0.108 & 0.017 & 171 & 5.01 \\ 98.79 & 0.047 & -0.088 & 0.051 & 74 & 6.24 \\ 99.10 & 0.041 & -0.044 & -0.114 & 53 & 6.78 \\ 99.96 & 0.033 & -0.026 & -0.066 & 132 & 5.33 \\ 99.10 & 0.027 & -0.015 & 0.118 & 26 & 8.17 \\ 99.31 & 0.021 & 0.105 & -0.054 & 77 & 6.20 \\ 99.88 & -0.099 & 0.007 & -0.033 & 168 & 5.05 \\ 99.61 & 0.001 & -0.095 & 0.085 & 71 & 6.29 \\ 99.76 & 0.078 & 0.045 & -0.079 & 204 & 4.64 \\ 99.19 & -0.032 & 0.034 & -0.029 & 50 & 6.91 \\ 99.81 & -0.048 & 0.014 & 0.058 & 1 & 14.64 \\ 99.42 & 0.037 & 0.091 & 0.027 & 153 & 5.18 \\ 99.12 & -0.071 & -0.084 & 0.026 & 67 & 6.39 \\ 99.16 & 0.068 & 0.019 & -0.078 & 32 & 7.87 \\ 99.76 & 0.047 & -0.017 & 0.101 & 86 & 6.04 \\ 99.84 & 0.078 & -0.067 & -0.015 & 212 & 4.57 \\ 99.70 & 0.052 & -0.015 & -0.118 & 113 & 5.45 \\ 99.93 & -0.106 & 0.003 & -0.005 & 256 & 3.85 \\ 99.95 & 0.003 & -0.028 & 0.045 & 16 & 9.03 \\ 99.79 & 0.043 & -0.052 & 0.117 & 141 & 5.23 \\ 99.68 & 0.027 & 0.035 & 0.106 & 128 & 5.35 \\ 99.80 & -0.103 & -0.023 & 0.001 & 241 & 4.14 \\ 99.91 & -0.095 & 0.045 & 0.030 & 210 & 4.58 \\ 99.74 & -0.072 & -0.060 & -0.008 & 92 & 5.97 \\ 99.88 & -0.104 & -0.021 & -0.002 & 234 & 4.23 \\ 99.23 & -0.090 & -0.029 & 0.047 & 97 & 5.78\end{array}$


1-Octen-3-ol

trans,trans-2,4-Heptadienal

$$
\text { 1-Heptanol }
$$

cis,cis-3,5-Octadiene

Methyl 2-Oxopropanoate

Dihydromyrcenol

trans-Menthone

$$
\text { Furfural }
$$

Ethanoic Acid

2-Ethyl-1-Hexanol

$$
\text { Decanal }
$$

Furfuryl Formate

trans-2-Hepten-1-ol

2-Nonanol

1-(2-Furanyl)-Ethanone

3,5-Octadien-2-one

Benzaldehyde

Furfuryl Acetate

Linalool

$\beta$-Myrcene

1-Octanol

trans-p-2-Menthen-1-ol

Propanoic Acid

2,3-Butanediol

Dimethyl Sulfoxide

m-Menth-3-ene

Trans-(2-Ethylcyclopentyl)-Methanol

Bornyl Acetate

4-Cyclopentene-1,3-dione

Menthol

$\begin{array}{ccccc}\text { OCT3E1OL } & -0.893 & -0.179 & 0.079 & 232 \\ \text { TTHPT22DEAL } & 0.605 & -0.075 & -0.158 & 107 \\ \text { HPT1OL } & 0.805 & 0.049 & -0.190 & 203 \\ \text { CCOCT35DENE } & 0.589 & -0.246 & -0.444 & 261 \\ \text { OM2ESTPPA } & 0.509 & -0.710 & 0.283 & 160 \\ \text { DHMYRCNOL } & 0.440 & -0.498 & 0.581 & 269 \\ \text { TMTHONE } & -0.926 & -0.198 & 0.085 & 79 \\ \text { FURAL } & -0.515 & -0.127 & -0.059 & 33 \\ \text { ETNOIC } & 0.753 & -0.112 & 0.451 & 250 \\ \text { E2HX1OL } & -0.758 & 0.035 & 0.558 & 187 \\ \text { DECAL } & 0.000 & 0.527 & 0.408 & 228 \\ \text { FURYLFMTE } & 0.640 & -0.231 & 0.558 & 70 \\ \text { THPT1E2OL } & 0.466 & 0.499 & 0.337 & 190 \\ \text { NON2OL } & 0.299 & -0.230 & -0.891 & 16 \\ \text { FURYLETONE } & 0.432 & -0.417 & 0.062 & 54 \\ \text { OCT35DE2ONE } & -0.791 & 0.293 & 0.360 & 162 \\ \text { BENZAL } & -0.606 & 0.436 & 0.340 & 270 \\ \text { FURYLACTE } & -0.599 & 0.146 & 0.165 & 128 \\ \text { LINOL } & 0.435 & 0.212 & 0.455 & 141 \\ \text { BMYRCNE } & -0.967 & -0.055 & -0.124 & 51 \\ \text { OCT1OL } & 0.725 & 0.573 & 0.145 & 245 \\ \text { TMNTH1E2OL } & -0.781 & -0.225 & 0.192 & 43 \\ \text { PPANOIC } & -0.241 & -0.281 & 0.489 & 59 \\ \text { BT23DIOL } & 0.493 & -0.449 & -0.259 & 169 \\ \text { DMSULFO } & 0.660 & -0.352 & 0.237 & 165 \\ \text { MMTH3ENE } & 0.589 & -0.607 & 0.353 & 58 \\ \text { T2ECYPTMTOL } & 0.805 & 0.336 & -0.115 & 239 \\ \text { BORNYLACTE } & 0.817 & 0.263 & 0.130 & 189 \\ \text { CYPT4E13DONE } & 0.445 & -0.299 & 0.402 & 39 \\ \text { MNTHOL } & -0.942 & -0.255 & -0.065 & 65\end{array}$

(9)

5.02 
2-Undecanone

2-(2-Furanyl)-Furan trans-2-Octen-1-ol trans-2-Nonen-1-ol

Diethylene Glycol Ethyl Ether

cis-p-2-Menthen-1-ol

$\beta$-Cyclocitral

Benzeneacetaldehyde

1-Nonanol

Furfuryl Alcohol

Phoracantho

cis-3-Nonen-1-ol

cis-Piperitol

p-Methoxy-Styrene

(2Z)-3-Ethyl-2,4-Pentadien-1-ol

Butylene Glycol Monobutyl Ether $\alpha$-Terpineol

1,5-Cyclooctanedione $\gamma$-Terpinene

trans-2-Decenol

2-Propenoic Acid

2-Undecanol

3-Ethyl-Benzaldehyde

3-Hydroxy-Benzaldehyde trans-2-Nonene

2-Methyl-2-Pentenal cis-5-Decenol trans-Piperitol

4-Ethyl-Benzaldehyde Naphthalene

$\begin{array}{cccc}\text { UNDEC2ONE } & 0.356 & -0.159 & -0.103 \\ \text { FURYLFUR } & 0.578 & 0.403 & 0.382 \\ \text { TOCT1E2OL } & 0.577 & 0.771 & 0.151 \\ \text { TNON1E2OL } & -0.959 & 0.068 & 0.128 \\ \text { DENGOLEETHR } & -0.040 & 0.292 & 0.811 \\ \text { CMNTH1E2OL } & 0.109 & -0.092 & 0.218 \\ \text { BCYCITAL } & -0.893 & 0.239 & 0.049 \\ \text { BENZACETAL } & -0.441 & -0.515 & 0.459 \\ \text { NON1OL } & 0.571 & 0.027 & -0.709 \\ \text { FURFOL } & 0.007 & 0.269 & 0.420 \\ \text { PHOCATHOL } & 0.358 & -0.430 & 0.572 \\ \text { ZNONE3OL } & 0.130 & -0.424 & 0.565 \\ \text { CPIPETOL } & -0.770 & -0.274 & 0.211 \\ \text { PMTXYESTYNE } & 0.058 & 0.890 & 0.401 \\ \text { E3PT1DE24OL } & 0.796 & -0.094 & 0.536 \\ \text { BNGOLBTHR } & 0.864 & -0.231 & 0.301 \\ \text { ATERPINOL } & -0.835 & -0.284 & 0.162 \\ \text { CYOCTD15ONE } & -0.687 & -0.169 & -0.128 \\ \text { GTERPINE } & 0.410 & 0.823 & -0.334 \\ \text { TDECE2OL } & 0.616 & 0.497 & 0.328 \\ \text { PPE2NOIC } & 0.215 & -0.430 & 0.559 \\ \text { UNDEC2OL } & 0.316 & -0.219 & -0.568 \\ \text { E3BNZAL } & -0.571 & 0.193 & 0.678 \\ \text { HXY3BENZAL } & 0.395 & -0.298 & 0.829 \\ \text { TNONENE } & 0.231 & -0.649 & -0.050 \\ \text { M2PNTE2AL } & -0.017 & -0.478 & 0.832 \\ \text { CDEC5E1OL } & 0.271 & -0.241 & -0.848 \\ \text { TPIPETOL } & -0.760 & -0.287 & 0.181 \\ \text { E4BNZAL } & -0.552 & 0.702 & 0.299 \\ \text { NPHNE } & 0.683 & 0.452 & 0.511\end{array}$

186

255

183

196

258

71

121

212

80

246

18

263

19

193

236

235

63

194

204

237

46

62

260

93

231

206

175

34

244

157 $\begin{array}{llll}99.52 & 0.039 & -0.020 & 0.013\end{array}$

$\begin{array}{llll}98.70 & 0.061 & 0.054 & -0.059\end{array}$

$\begin{array}{lllll}99.55 & 0.060 & 0.102 & -0.021\end{array}$

$\begin{array}{lllll}99.45 & -0.103 & 0.007 & -0.019\end{array}$

$\begin{array}{llll}98.60 & -0.005 & 0.039 & -0.123\end{array}$

$\begin{array}{lllll}99.88 & 0.012 & -0.011 & -0.031\end{array}$

$\begin{array}{lllll}99.78 & -0.096 & 0.029 & -0.006\end{array}$

$\begin{array}{lllll}99.31 & -0.046 & -0.067 & -0.072\end{array}$

$\begin{array}{llll}99.85 & 0.061 & 0.004 & 0.109\end{array}$

$\begin{array}{lllll}98.94 & 0.000 & 0.035 & -0.066\end{array}$

$\begin{array}{lllll}99.95 & 0.039 & -0.054 & -0.089\end{array}$

$\begin{array}{lllll}98.22 & 0.015 & -0.053 & -0.085\end{array}$

$\begin{array}{lllll}99.95 & -0.082 & -0.037 & -0.031\end{array}$

$\begin{array}{lllll}99.48 & 0.004 & 0.116 & -0.060\end{array}$

$\begin{array}{lllll}99.06 & 0.086 & -0.009 & -0.082\end{array}$

$\begin{array}{lllll}99.06 & 0.093 & -0.027 & -0.047\end{array}$

$\begin{array}{lllll}99.90 & -0.089 & -0.039 & -0.027\end{array}$

$\begin{array}{lllll}99.47 & -0.073 & -0.024 & 0.020\end{array}$

$\begin{array}{lllll}99.40 & 0.042 & 0.107 & 0.053\end{array}$

$\begin{array}{lllll}99.04 & 0.065 & 0.067 & -0.048\end{array}$

$\begin{array}{lllll}99.92 & 0.024 & -0.054 & -0.087\end{array}$

$\begin{array}{lllll}99.90 & 0.035 & -0.029 & 0.084\end{array}$

$\begin{array}{lllll}98.55 & -0.062 & 0.025 & -0.103\end{array}$

$\begin{array}{lllll}99.83 & 0.043 & -0.036 & -0.126\end{array}$

$\begin{array}{lllll}99.10 & 0.027 & -0.084 & 0.004\end{array}$

$\begin{array}{lllll}99.39 & -0.001 & -0.060 & -0.128\end{array}$

$\begin{array}{lllll}99.61 & 0.030 & -0.033 & 0.128\end{array}$

$\begin{array}{lllll}99.94 & -0.081 & -0.038 & -0.027\end{array}$

$\begin{array}{lllll}98.97 & -0.061 & 0.090 & -0.044\end{array}$

$\begin{array}{lllll}99.67 & 0.072 & 0.062 & -0.076\end{array}$

$\begin{array}{cc}69 & 6.36 \\ 116 & 5.43 \\ 236 & 4.22 \\ 251 & 3.89 \\ 27 & 8.06 \\ 4 & 13.10 \\ 225 & 4.40 \\ 197 & 4.73 \\ 182 & 4.92 \\ 84 & 6.09 \\ 191 & 4.81 \\ 81 & 6.12 \\ 230 & 4.32 \\ 229 & 4.33 \\ 231 & 4.31 \\ 161 & 5.09 \\ 218 & 4.51 \\ 3 & 13.60 \\ 209 & 4.58 \\ 160 & 5.10 \\ 104 & 5.69 \\ 109 & 5.57 \\ 42 & 7.26 \\ 162 & 5.09 \\ 155 & 5.15 \\ 179 & 4.93 \\ 164 & 5.08 \\ 223 & 4.46 \\ 177 & 4.94 \\ 200 & 4.70\end{array}$


1-Decanol

Cyclodecane

trans,trans-2,4-Decadienal

1,2-Cyclopentanedione

Cyclodecene

cis-4-Decenol

1-Phenyl-2-Butanone

Phenethyl Alcohol

trans,cis-2,4-Decadienal

2-Cyclohexenol

2-Ethyl Hexanoic Acid

3-Methyl-1,2-Cyclopentanedione

trans-Cinnamaldehyde

Heptanoic Acid

$$
\text { Mequinol }
$$

2,4,5-Trihydroxypyrimidine cis-Cinnamaldehyde

Benzenemethanol

Mesitaldehyde

2-Ethylhexyl Octanoate

Benzeneethanol

1,4-Butanediol

$\beta$-Ionone

2-Methyl-1,4-Benzenediol

2-Ethyl Heptanoic Acid

1-Dodecanol

Benzalacetone

Maltol

2-Acetylpyrrole

2,4-Decadien-1-ol

$\begin{array}{cccc}\text { DEC1OL } & 0.188 & -0.398 & 0.379 \\ \text { CDECANE } & 0.474 & -0.303 & 0.637 \\ \text { TTDEC24DENAL } & 0.410 & 0.895 & 0.056 \\ \text { CPT12DONE } & 0.469 & -0.612 & 0.564 \\ \text { CDECENE } & 0.784 & -0.180 & 0.295 \\ \text { CDEC4E1OL } & -0.427 & -0.543 & -0.288 \\ \text { PHYLBT2ONE } & 0.045 & 0.799 & -0.295 \\ \text { MBNZMETOL } & 0.081 & -0.321 & 0.709 \\ \text { TCDEC24DENAL } & 0.321 & 0.937 & 0.073 \\ \text { CHEX2E1OL } & 0.508 & -0.607 & 0.587 \\ \text { E2HEXOIC } & -0.020 & 0.751 & 0.638 \\ \text { M3CPT12DONE } & 0.454 & -0.394 & 0.369 \\ \text { TCINAMAL } & -0.047 & -0.086 & 0.962 \\ \text { HEPTOIC } & -0.624 & 0.170 & 0.717 \\ \text { MEQNOL } & 0.452 & 0.445 & 0.288 \\ \text { THDXYPYMNE } & 0.175 & -0.459 & 0.511 \\ \text { CCINAMAL } & -0.160 & -0.219 & 0.734 \\ \text { BENZMTOL } & 0.378 & 0.760 & 0.404 \\ \text { MESIAL } & -0.400 & 0.287 & 0.752 \\ \text { EHESTOA } & 0.012 & -0.331 & -0.031 \\ \text { BENZETOL } & 0.223 & -0.317 & 0.827 \\ \text { BT14DIOL } & 0.364 & -0.299 & 0.760 \\ \text { BIONNE } & -0.852 & 0.417 & 0.155 \\ \text { M2BNZ14DIOL } & 0.571 & -0.530 & 0.559 \\ \text { EHPTOIC } & 0.288 & -0.086 & -0.857 \\ \text { DODEC1OL } & -0.520 & 0.331 & 0.355 \\ \text { BNZONE } & -0.040 & -0.273 & 0.808 \\ \text { MALTOL } & 0.294 & -0.349 & 0.553 \\ \text { ACTLPYROLE } & 0.467 & -0.615 & 0.320 \\ \text { DEC24DIE1OL } & 0.907 & -0.062 & 0.116\end{array}$

233

221

171

83

202

226

145

53

67

74

138

166

131

205

268

254

249

134

164

262

197

103

238

217

220

23

127

218

$\begin{array}{lccccc}99.07 & 0.021 & -0.051 & -0.059 & 68 & 6.38 \\ 99.19 & 0.052 & -0.037 & -0.099 & 174 & 4.99 \\ 99.62 & 0.042 & 0.117 & -0.007 & 242 & 4.10 \\ 99.85 & 0.052 & -0.077 & -0.087 & 232 & 4.29 \\ 99.41 & 0.085 & -0.020 & -0.044 & 207 & 4.62 \\ 99.42 & -0.044 & -0.072 & 0.041 & 134 & 5.32 \\ 99.13 & 0.003 & 0.103 & 0.047 & 76 & 6.20 \\ 99.70 & 0.009 & -0.039 & -0.106 & 176 & 4.94 \\ 99.87 & 0.032 & 0.122 & -0.009 & 247 & 4.01 \\ 99.91 & 0.056 & -0.076 & -0.090 & 228 & 4.34 \\ 99.88 & -0.004 & 0.098 & -0.096 & 226 & 4.39 \\ 99.87 & 0.050 & -0.049 & -0.057 & 150 & 5.20 \\ 99.74 & -0.005 & -0.009 & -0.146 & 83 & 6.10 \\ 99.64 & -0.067 & 0.022 & -0.109 & 219 & 4.51 \\ 99.76 & 0.047 & 0.060 & -0.041 & 120 & 5.39 \\ 99.40 & 0.020 & -0.058 & -0.080 & 151 & 5.18 \\ 97.65 & -0.017 & -0.027 & -0.113 & 106 & 5.64 \\ 98.72 & 0.038 & 0.100 & -0.059 & 185 & 4.90 \\ 98.82 & -0.044 & 0.038 & -0.112 & 121 & 5.39 \\ 99.75 & 0.002 & -0.042 & 0.007 & 115 & 5.44 \\ 99.65 & 0.025 & -0.039 & -0.126 & 175 & 4.98 \\ 98.25 & 0.040 & -0.036 & -0.118 & 136 & 5.29 \\ 99.44 & -0.093 & 0.052 & -0.022 & 227 & 4.39 \\ 99.81 & 0.063 & -0.066 & -0.086 & 126 & 5.35 \\ 99.02 & 0.031 & -0.012 & 0.131 & 75 & 6.23 \\ 99.22 & -0.057 & 0.042 & -0.055 & 59 & 6.49 \\ 99.19 & -0.004 & -0.034 & -0.125 & 135 & 5.32 \\ 99.95 & 0.032 & -0.043 & -0.085 & 85 & 6.09 \\ 99.76 & 0.052 & -0.078 & -0.052 & 190 & 4.82 \\ 99.22 & 0.097 & -0.005 & -0.017 & 103 & 5.69\end{array}$




\begin{tabular}{|c|c|c|c|c|c|c|c|c|c|c|c|c|}
\hline 234 & Levoglucosenone & LEVGLCONE & 0.425 & -0.135 & 0.463 & 213 & 99.31 & 0.046 & -0.015 & -0.071 & 58 & 6.51 \\
\hline 235 & Phenol & PHEOL & 0.003 & 0.609 & -0.067 & 267 & 97.78 & -0.001 & 0.078 & 0.010 & 37 & 7.64 \\
\hline 236 & 1-(2-Furanyl)-2-Hydroxy-Ethanone & FURYLHXYEONE & 0.488 & -0.594 & 0.072 & 87 & 99.84 & 0.054 & -0.076 & -0.014 & 122 & 5.38 \\
\hline 237 & 4,6-Dimethyl-Dodecane & DM45DODCANE & 0.299 & -0.590 & 0.371 & 153 & 99.68 & 0.034 & -0.075 & -0.058 & 124 & 5.37 \\
\hline 238 & Ethyl Decanoate & EESDECA & 0.799 & -0.032 & -0.382 & 113 & 99.79 & 0.086 & -0.003 & 0.059 & 222 & 4.46 \\
\hline 239 & 1-Methyl-4-Piperidinone & M1PIP4DIONE & -0.890 & -0.362 & 0.204 & 152 & 99.69 & -0.094 & -0.049 & -0.031 & 258 & 3.83 \\
\hline 240 & 2,2-Dimethyl-3-Heptanone & DM22HPT3ONE & 0.154 & -0.289 & 0.104 & 104 & 99.81 & 0.017 & -0.037 & -0.016 & 49 & 6.91 \\
\hline 241 & Octanoic Acid & OCTOIC & -0.113 & -0.098 & 0.881 & 216 & 99.22 & -0.012 & -0.011 & -0.134 & 142 & 5.23 \\
\hline 242 & 1,3-Dihydroxy-2-Propanone & DHYPPAONE & 0.429 & -0.422 & 0.581 & 177 & 99.59 & 0.047 & -0.052 & -0.088 & 186 & 4.89 \\
\hline 243 & 2-Phenoxy-Ethanol & PHENXYOL & -0.969 & 0.011 & 0.024 & 105 & 99.81 & -0.104 & -0.001 & -0.003 & 259 & 3.77 \\
\hline 244 & Isophthalaldehyde & ISOPHTAL & -0.821 & 0.078 & 0.307 & 271 & 96.23 & -0.088 & 0.008 & -0.048 & 183 & 4.92 \\
\hline 245 & Cyclotetradecane & CTTDECANE & 0.352 & -0.184 & 0.869 & 66 & 99.89 & 0.038 & -0.021 & -0.132 & 163 & 5.09 \\
\hline 246 & Nonanoic Acid & NONOIC & -0.785 & 0.441 & 0.411 & 97 & 99.82 & -0.085 & 0.056 & -0.062 & 252 & 3.88 \\
\hline 247 & 2-Ethyl-Phenol & E2PHEOL & 0.480 & 0.349 & 0.188 & 265 & 98.11 & 0.050 & 0.047 & -0.025 & 129 & 5.35 \\
\hline 248 & 2-Methoxy-4-Vinyl-Phenol & MXY2VYL4PHEOL & 0.862 & -0.335 & 0.011 & 143 & 99.72 & 0.093 & -0.041 & -0.001 & 254 & 3.86 \\
\hline 249 & 4-Methylimidazole & M4IMDZOLE & -0.968 & 0.025 & -0.029 & 150 & 99.69 & -0.104 & 0.001 & 0.005 & 255 & 3.86 \\
\hline 250 & m-tert-Butyl-Toluene & MTBTLNE & -0.184 & 0.249 & 0.526 & 251 & 98.80 & -0.020 & 0.032 & -0.082 & 40 & 7.27 \\
\hline 251 & Syringol & SYNGOL & 0.390 & -0.520 & 0.602 & 266 & 97.81 & 0.043 & -0.065 & -0.093 & 62 & 6.46 \\
\hline 252 & Decanoic Acid & DECOIC & -0.421 & 0.130 & 0.872 & 208 & 99.36 & -0.046 & 0.018 & -0.132 & 180 & 4.92 \\
\hline 253 & 3-Hydroxy-2,3-Dihydro-Maltol & HX3DH23MALTOL & 0.409 & 0.076 & 0.778 & 112 & 99.79 & 0.044 & 0.012 & -0.119 & 194 & 4.78 \\
\hline 254 & 5-Hydroxy-Maltol & HX5MALTOL & -0.171 & -0.157 & 0.246 & 158 & 99.67 & -0.018 & -0.021 & -0.040 & 79 & 6.16 \\
\hline 255 & Triethylene Glycol & TETYNEGLOL & -0.034 & 0.166 & 0.398 & 56 & 99.91 & -0.004 & 0.023 & -0.058 & 118 & 5.42 \\
\hline 256 & Benzoic Acid & BNZOIC & 0.599 & -0.370 & -0.107 & 182 & 99.56 & 0.065 & -0.046 & 0.017 & 78 & 6.18 \\
\hline 257 & 2-Methyl-Benzaldehyde & M2BNZAL & 0.604 & 0.023 & -0.252 & 115 & 99.79 & 0.065 & 0.004 & 0.041 & 159 & 5.12 \\
\hline 258 & Undecanoic Acid & UNDECOIC & -0.852 & -0.004 & 0.506 & 130 & 99.76 & -0.091 & -0.002 & -0.077 & 249 & 3.94 \\
\hline 259 & 4-Pyridinol & PYRDINOL & -0.132 & -0.288 & 0.864 & 102 & 99.81 & -0.013 & -0.036 & -0.131 & 166 & 5.06 \\
\hline 260 & 4-Amino-Phenol & AMIPHEOL & 0.447 & -0.551 & 0.574 & 44 & 99.92 & 0.049 & -0.069 & -0.088 & 181 & 4.92 \\
\hline
\end{tabular}

${ }^{1}$ VIP - Variable Importance in Projection scores. 
Table S10. Loading results of samples and variables from Principal Component Analysis (PCA) and Partial Least Squares Analysis (PLS) based on the relative peak areas of the identified VOCs in sugarcane samples.

\begin{tabular}{|c|c|c|c|c|c|c|c|}
\hline \multirow{2}{*}{ ID Sample Code } & \multirow{2}{*}{ ID Variety Code } & \multicolumn{3}{|c|}{ PCA } & \multicolumn{3}{|c|}{ PLS } \\
\hline & & 1 & 2 & 3 & 1 & 2 & 3 \\
\hline AMA15 & AMA & 0.13 & -0.28 & 0.31 & n.a. & n.a. & n.a. \\
\hline AMA17 & AMA & 0.22 & -0.29 & 0.46 & n.a. & n.a. & n.a. \\
\hline RAD15 & RAD & 0.13 & -0.13 & -0.25 & n.a. & n.a. & n.a. \\
\hline RAD17 & RAD & 0.20 & -0.14 & -0.22 & n.a. & n.a. & n.a. \\
\hline ROX15 & $\mathrm{ROX}$ & 0.00 & 0.61 & 0.11 & n.a. & n.a. & n.a. \\
\hline ROX17 & ROX & 0.06 & 0.69 & 0.15 & n.a. & n.a. & n.a. \\
\hline VER15 & VER & 0.12 & -0.11 & 0.18 & n.a. & n.a. & n.a. \\
\hline VER17 & VER & 0.18 & -0.08 & 0.29 & n.a. & n.a. & n.a. \\
\hline VIO15 & VIO & 0.12 & -0.06 & -0.50 & n.a. & n.a. & n.a. \\
\hline VIO17 & VIO & 0.17 & 0.01 & -0.52 & n.a. & n.a. & n.a. \\
\hline CAN15 & CAN & -0.68 & -0.11 & 0.01 & n.a. & n.a. & n.a. \\
\hline CAN17 & CAN & -0.64 & -0.10 & -0.02 & n.a. & n.a. & n.a. \\
\hline Centroid (AMA) & AMA & n.a. & n.a. & n.a. & 0.03 & -0.05 & -0.09 \\
\hline Centroid (RAD) & RAD & n.a. & n.a. & n.a. & 0.03 & -0.03 & 0.05 \\
\hline Centroid (ROX) & ROX & n.a. & n.a. & n.a. & 0.00 & 0.12 & -0.03 \\
\hline Centroid (VER) & VER & n.a. & n.a. & n.a. & 0.02 & -0.02 & -0.05 \\
\hline Centroid (VIO) & VIO & n.a. & n.a. & n.a. & 0.02 & -0.01 & 0.12 \\
\hline Centroid (CAN) & CAN & n.a. & n.a. & n.a. & -0.10 & -0.02 & 0.00 \\
\hline
\end{tabular}

n.a. - not available

Table S11. Scores results of all cases from Principal Component Analysis (PCA) and Partial Least Squares Analysis (PLS) based on the relative peak areas of the identified VOCs in sugarcane samples. 


\begin{tabular}{|c|c|c|c|c|c|c|c|}
\hline \multirow{2}{*}{$\begin{array}{l}\text { ID Replicate } \\
\text { Number }\end{array}$} & \multirow{2}{*}{$\begin{array}{l}\text { ID Replicate } \\
\text { Code }\end{array}$} & \multicolumn{3}{|c|}{ PCA } & \multicolumn{3}{|c|}{ PLS } \\
\hline & & 1 & 2 & 3 & 1 & 2 & 3 \\
\hline 1 & AMA15A & 3.97 & -7.19 & 6.70 & 4.16 & -7.00 & -6.97 \\
\hline 2 & AMA15B & 3.93 & -7.08 & 6.55 & 4.11 & -6.90 & -6.81 \\
\hline 3 & AMA15C & 3.95 & -7.13 & 6.63 & 4.14 & -6.95 & -6.89 \\
\hline 4 & AMA17A & 5.87 & -6.99 & 8.23 & 6.02 & -6.75 & -8.40 \\
\hline 5 & AMA17B & 7.32 & -7.44 & 11.42 & 7.47 & -7.13 & -11.54 \\
\hline 6 & AMA17C & 6.60 & -7.22 & 9.83 & 6.75 & -6.94 & -9.97 \\
\hline 7 & RAD15A & 4.04 & -3.45 & -5.10 & 4.15 & -3.48 & 4.88 \\
\hline 8 & RAD15B & 4.14 & -3.41 & -5.69 & 4.24 & -3.45 & 5.47 \\
\hline 9 & RAD15C & 4.09 & -3.43 & -5.39 & 4.20 & -3.46 & 5.17 \\
\hline 10 & RAD17A & 6.31 & -3.60 & -4.66 & 6.39 & -3.57 & 4.46 \\
\hline 11 & RAD17B & 5.76 & -3.65 & -4.78 & 5.85 & -3.62 & 4.57 \\
\hline 12 & RAD17C & 6.03 & -3.62 & -4.72 & 6.12 & -3.60 & 4.51 \\
\hline 13 & ROX15A & 0.04 & 13.93 & 1.46 & -0.29 & 13.99 & -1.35 \\
\hline 14 & ROX15B & -0.10 & 16.75 & 3.47 & -0.50 & 16.81 & -3.29 \\
\hline 15 & ROX15C & -0.03 & 15.34 & 2.46 & -0.39 & 15.40 & -2.32 \\
\hline 16 & ROX17A & 1.51 & 16.07 & 2.58 & 1.10 & 16.14 & -2.40 \\
\hline 17 & ROX17B & 2.11 & 18.60 & 3.80 & 1.63 & 18.68 & -3.55 \\
\hline 18 & ROX17C & 1.81 & 17.33 & 3.19 & 1.37 & 17.41 & -2.98 \\
\hline 19 & VER15A & 3.86 & -2.76 & 4.27 & 3.93 & -2.57 & -4.16 \\
\hline 20 & VER15B & 3.27 & -2.92 & 3.34 & 3.36 & -2.76 & -3.26 \\
\hline 21 & VER15C & 3.56 & -2.84 & 3.80 & 3.65 & -2.67 & -3.71 \\
\hline 22 & VER17A & 5.42 & -2.23 & 5.81 & 5.45 & -2.00 & -5.62 \\
\hline 23 & VER17B & 5.77 & -1.96 & 6.47 & 5.80 & -1.72 & -6.22 \\
\hline 24 & VER17C & 5.59 & -2.10 & 6.14 & 5.62 & -1.86 & -5.92 \\
\hline 25 & VIO15A & 3.59 & -1.54 & -10.65 & 3.65 & -1.61 & 10.74 \\
\hline 26 & VIO15B & 3.53 & -1.41 & -10.70 & 3.58 & -1.48 & 10.77 \\
\hline 27 & VIO15C & 3.56 & -1.48 & -10.67 & 3.62 & -1.54 & 10.75 \\
\hline 28 & VIO17A & 5.12 & 0.26 & -11.24 & 5.12 & 0.16 & 11.35 \\
\hline
\end{tabular}




\begin{tabular}{lccccccc}
29 & VIO17B & 5.16 & 0.22 & -11.16 & 5.16 & 0.13 & 11.28 \\
30 & VIO17C & 5.14 & 0.24 & -11.20 & 5.14 & 0.15 & 11.32 \\
31 & CAN15A & -19.48 & -2.89 & -0.21 & -19.41 & -3.30 & 0.18 \\
32 & CAN15B & -22.09 & -2.36 & 0.73 & -22.02 & -2.82 & -0.71 \\
33 & CAN15C & -20.78 & -2.63 & 0.26 & -20.72 & -3.06 & -0.26 \\
34 & CAN17A & -19.37 & -2.57 & -0.61 & -19.32 & -2.99 & 0.56 \\
35 & CAN17B & -19.67 & -2.36 & -0.05 & -19.63 & -2.78 & 0.02 \\
36 & CAN17C & -19.52 & -2.46 & -0.33 & -19.48 & -2.89 & 0.29 \\
\hline
\end{tabular}

Table S12. Canonical Discriminant Function Coefficients and Highest Probability Classification results of samples from Linear Discriminant Analysis (LDA) after matrix reduction method obtained from One-way ANOVA test based on the relative peak areas of identified VOCs in sugarcane samples.

\begin{tabular}{|c|c|c|c|c|c|c|c|c|c|c|}
\hline \multirow{3}{*}{$\begin{array}{c}\text { ID } \\
\text { Replicate } \\
\text { Code }\end{array}$} & \multirow{3}{*}{$\begin{array}{c}\text { ID } \\
\text { Variety } \\
\text { Code }\end{array}$} & \multicolumn{9}{|c|}{ LDA } \\
\hline & & \multicolumn{3}{|c|}{$\mathrm{CDF}^{1}$} & \multicolumn{3}{|c|}{$\mathrm{CDF}^{1}$ Class Mean } & \multicolumn{3}{|c|}{$\begin{array}{c}\text { Highest Probability } \\
\text { Classification }\end{array}$} \\
\hline & & 1 & 2 & 3 & 1 & 2 & 3 & First & Second & Third \\
\hline AMA15A & & 4761.06 & 1257.08 & 2488.92 & & & & AMA & ROX & VER \\
\hline AMA15B & AMA & 4761.10 & 1257.14 & 2488.90 & 20 & 23 & 64 & AMA & ROX & VER \\
\hline
\end{tabular}




\begin{tabular}{|c|c|c|c|c|c|c|c|c|c|c|}
\hline AMA15C & & 4758.43 & 1257.46 & 2488.07 & & & & AMA & ROX & VER \\
\hline AMA17A & & 4761.10 & 1257.78 & 2488.49 & & & & AMA & ROX & VER \\
\hline AMA17B & & 4761.12 & 1257.82 & 2488.43 & & & & AMA & ROX & VER \\
\hline AMA17C & & 4758.39 & 1256.10 & 2489.02 & & & & AMA & ROX & VER \\
\hline RAD15A & & 3911.76 & 2210.37 & -309.59 & \multirow{6}{*}{3910.84} & \multirow{6}{*}{2210.20} & \multirow{6}{*}{-309.34} & RAD & ROX & VER \\
\hline RAD15B & \multirow{5}{*}{ RAD } & 3911.79 & 2210.40 & -309.69 & & & & RAD & ROX & VER \\
\hline RAD15C & & 3908.97 & 2209.85 & -308.73 & & & & RAD & ROX & VER \\
\hline RAD17A & & 3911.93 & 2210.73 & -309.64 & & & & RAD & ROX & VER \\
\hline RAD17B & & 3911.99 & 2210.78 & -309.62 & & & & RAD & ROX & VER \\
\hline RAD17C & & 3908.57 & 2209.08 & -308.77 & & & & RAD & ROX & VER \\
\hline ROX15A & \multirow{6}{*}{ ROX } & -3224.84 & 839.81 & 1128.66 & \multirow{6}{*}{-3225.08} & \multirow{6}{*}{840.17} & \multirow{6}{*}{1127.53} & ROX & RAD & VER \\
\hline ROX15B & & -3224.85 & 839.81 & 1128.66 & & & & ROX & RAD & VER \\
\hline ROX15C & & -3225.53 & 840.91 & 1125.26 & & & & ROX & RAD & VER \\
\hline ROX17A & & -3225.31 & 840.37 & 1128.06 & & & & ROX & RAD & VER \\
\hline ROX17B & & -3225.32 & 840.33 & 1128.10 & & & & ROX & RAD & VER \\
\hline ROX17C & & -3224.60 & 839.81 & 1126.45 & & & & ROX & RAD & VER \\
\hline VER15A & \multirow{6}{*}{ VER } & -1884.33 & 3365.55 & -2672.50 & \multirow{6}{*}{-1884.36} & \multirow{6}{*}{3364.28} & \multirow{6}{*}{-2672.69} & VER & ROX & RAD \\
\hline VER15B & & -1884.34 & 3365.57 & -2672.54 & & & & VER & ROX & RAD \\
\hline VER15C & & -1884.38 & 3361.76 & -2673.06 & & & & VER & ROX & RAD \\
\hline VER17A & & -1883.76 & 3363.48 & -2673.56 & & & & VER & ROX & RAD \\
\hline VER17B & & -1883.76 & 3363.52 & -2673.63 & & & & VER & ROX & RAD \\
\hline VER17C & & -1885.60 & 3365.81 & -2670.84 & & & & VER & ROX & RAD \\
\hline VIO15A & \multirow{6}{*}{ VIO } & 4543.55 & -5357.89 & -1357.82 & \multirow{6}{*}{4543.66} & \multirow{6}{*}{-5357.13} & \multirow{6}{*}{-1357.94} & $\mathrm{VIO}$ & ROX & VER \\
\hline VIO15B & & 4543.59 & -5357.81 & -1357.92 & & & & VIO & ROX & VER \\
\hline VIO15C & & 4543.82 & -5355.68 & -1358.06 & & & & VIO & ROX & VER \\
\hline VIO17A & & 4544.39 & -5357.78 & -1356.69 & & & & VIO & ROX & VER \\
\hline VIO17B & & 4544.36 & -5357.80 & -1356.61 & & & & VIO & ROX & VER \\
\hline VIO17C & & 4542.25 & -5355.81 & -1360.55 & & & & VIO & ROX & VER \\
\hline CAN15A & \multirow{2}{*}{ CAN } & -8105.75 & -2314.18 & 723.82 & \multirow[t]{2}{*}{-8105.26} & \multirow[t]{2}{*}{-2314.76} & \multirow[t]{2}{*}{723.80} & CAN & ROX & VER \\
\hline CAN15B & & -8105.77 & -2314.20 & 723.84 & & & & CAN & ROX & VER \\
\hline
\end{tabular}




\begin{tabular}{lllllll} 
CAN15C & -8104.27 & -2315.90 & 723.73 & CAN & ROX & VER \\
CAN17A & -8105.18 & -2313.94 & 724.55 & CAN & ROX & VER \\
CAN17B & -8105.18 & -2313.94 & 724.55 & CAN & ROX & VER \\
CAN17C & -8105.42 & -2316.39 & 722.29 & CAN & ROX & VER \\
\hline
\end{tabular}

${ }^{1} \mathrm{CDF}$ - Canonical Discriminant Function Coefficients.

Table S13. Information summary of Partial Least Square Analysis (PLS) based only on the relative peak areas of the 23 most predictive VOCs identified in sugarcane samples.

\begin{tabular}{cccccccc}
\hline Analysis & Component & $\mathbf{R}^{\mathbf{2}} \mathbf{X}$ & Eigenvalues & $\mathbf{R}^{\mathbf{2}} \mathbf{Y}$ & $\mathbf{Q}^{\mathbf{2}}$ & Significance & Iterations \\
\hline & 1 & 0,4137 & 11,997 & 0,0908 & 0,0292 & $\mathrm{~S}$ & 10 \\
& 2 & 0,2058 & 5,969 & 0,0909 & 0,0287 & $\mathrm{~S}$ & 23 \\
& 3 & 0,1638 & 4,750 & 0,0909 & 0,0365 & $\mathrm{~S}$ & 20 \\
& 4 & 0,1281 & 3,714 & 0,0908 & 0,0571 & $\mathrm{~S}$ & 7 \\
& 5 & 0,0806 & 2,337 & 0,0909 & 0,0856 & $\mathrm{~S}$ & 3 \\
& 6 & 0,0023 & 0,062 & 0,0694 & 0,0410 & $\mathrm{~S}$ & 12 \\
& 6 & 0,0019 & 0,055 & 0,0803 & 0,1272 & $\mathrm{~S}$ & 7 \\
& 7 & 0,0010 & 0,029 & 0,0854 & 0,1699 & $\mathrm{~S}$ & 16 \\
& 8 & 0,0006 & 0,018 & 0,0777 & 0,1586 & $\mathrm{~S}$ & 14 \\
& 9 & 0,0004 & 0,013 & 0,0788 & 0,2370 & $\mathrm{~S}$ & 9 \\
& 10 & 0,0005 & 0,010 & 0,0503 & $-0,0589$ & $\mathrm{~S}$ & 15 \\
& 11 & 0,0003 & 0,009 & 0,0446 & 0,3147 & $\mathrm{~S}$ & 5 \\
& 12 & 0,0002 & 0,005 & 0,0115 & $-0,0270$ & $\mathrm{~S}$ & 21 \\
& 13 & 0,0002 & 0,005 & 0,0079 & 0,0002 & $\mathrm{~S}$ & 9 \\
\hline
\end{tabular}


Table S14. Results of variables from Linear Discriminant Analysis (LDA) after matrix reduction method to $20 \%$ of original dimension according with higher on the variable importance in projection (VIP) scores values from the PLS analysis based on the relative peak areas of the identified VOCs in sugarcane samples.

\begin{tabular}{|c|c|c|c|c|c|}
\hline \multirow{3}{*}{ Volatile Organic Compounds } & \multirow{3}{*}{ Abbreviations } & \multirow{2}{*}{\multicolumn{2}{|c|}{$\begin{array}{l}\text { PLS } \\
\text { VIP }^{1} \\
\end{array}$}} & \multicolumn{2}{|c|}{ LDA } \\
\hline & & & & \multirow[b]{2}{*}{$\mathbf{W}^{2}$} & \multirow[b]{2}{*}{$\mathbf{F}^{3}$} \\
\hline & & Importance & $\begin{array}{l}\text { Power } \\
\text { (x 100) }\end{array}$ & & \\
\hline 2-Methyl-2-Cyclopenten-1-one & M2CY2PT1EONE & 1 & 14.64 & \multicolumn{2}{|c|}{ Removed from analysis. } \\
\hline Ethyl Ether & EETHR & 2 & 13.71 & $3.82 \mathrm{E}-67$ & $5.90 \mathrm{E}+66$ \\
\hline 1.5-Cyclooctanedione & CYOCTD15ONE & 3 & 13.60 & \multicolumn{2}{|c|}{ Removed from analysis. } \\
\hline cis-p-2-Menthen-1-ol & CMNTH1E2OL & 4 & 13.10 & \multicolumn{2}{|c|}{ Removed from analysis. } \\
\hline 2-Ethyl-Furan & E2FUR & 5 & 13.06 & 7.33E-73 & $4.55 \mathrm{E}+72$ \\
\hline Furfural & FURAL & 6 & 12.44 & \multicolumn{2}{|c|}{ Removed from analysis. } \\
\hline 2-Methyl-1-Pentene & M2PT1ENE & 7 & 11.84 & \multicolumn{2}{|c|}{ Removed from analysis. } \\
\hline Furfuryl Acetate & FURYLACTE & 8 & 11.39 & \multicolumn{2}{|c|}{ Removed from analysis. } \\
\hline Methyl Acetate & MESTAA & 9 & 10.64 & $2.54 \mathrm{E}-66$ & $8.85 \mathrm{E}+65$ \\
\hline 1.4-Pentadiene & PT14DIENE & 10 & 10.63 & $1.93 \mathrm{E}-66$ & $1.16 \mathrm{E}+66$ \\
\hline cis-4-Methyl-2-Pentene & CM4PT2ENE & 11 & 9.87 & \multicolumn{2}{|c|}{ Removed from analysis. } \\
\hline Crotonal & CROTNAL & 12 & 9.83 & 2.02E-59 & $7.93 \mathrm{E}+58$ \\
\hline cis-3-Methyl-2-Pentene & CM3PT2ENE & 13 & 9.66 & $1.67 \mathrm{E}-66$ & $1.35 \mathrm{E}+66$ \\
\hline trans-2-Pentenol & TPT1E2OL & 14 & 9.33 & \multicolumn{2}{|c|}{ Removed from analysis. } \\
\hline Furan & FUR & 15 & 9.23 & $1.40 \mathrm{E}-66$ & $1.60 \mathrm{E}+66$ \\
\hline 3-Octen-2-one & OCT2E3ONE & 16 & 9.03 & \multicolumn{2}{|c|}{ Removed from analysis. } \\
\hline trans-Piperylene & TPIPERLNE & 17 & 8.91 & $3.22 \mathrm{E}-73$ & $1.04 \mathrm{E}+73$ \\
\hline 4-Methyl-Decane & M4DCANE & 18 & 8.83 & $5.05 \mathrm{E}-73$ & $6.60 \mathrm{E}+72$ \\
\hline 2.4-Dimethyl-Heptane & DM24HPTANE & 19 & 8.78 & \multicolumn{2}{|c|}{ Removed from analysis. } \\
\hline 4-Methyl-4-Heptanol & M4HPT4OL & 20 & 8.65 & \multicolumn{2}{|c|}{ Removed from analysis. } \\
\hline 2-Hexanone & HX2ONE & 21 & 8.45 & $1.01 \mathrm{E}-72$ & $3.31 \mathrm{E}+72$ \\
\hline 3-Hydroxy-2-Butanone & HXY3BT2ONE & 22 & 8.41 & \multicolumn{2}{|c|}{ Removed from analysis. } \\
\hline
\end{tabular}




\begin{tabular}{|c|c|c|c|c|c|}
\hline Benzene & $\mathrm{BNZ}$ & 23 & 8.24 & $2.43 \mathrm{E}-66$ & $9.26 \mathrm{E}+65$ \\
\hline cis.cis-3.5-Octadiene & CCOCT35DENE & 24 & 8.19 & $1.25 \mathrm{E}-66$ & $1.79 \mathrm{E}+66$ \\
\hline 2-Methyl-Furan & M2FUR & 25 & 8.19 & \multicolumn{2}{|c|}{ Removed from analysis. } \\
\hline 5-Methyl-4-Hexen-3-one & M5HX3E4ONE & 26 & 8.17 & \multicolumn{2}{|c|}{ Removed from analysis. } \\
\hline iethylene Glycol Ethyl Ether & DENGOLEETHR & 27 & 8.06 & \multicolumn{2}{|c|}{ Removed from analysis. } \\
\hline 4-Methyl-Heptane & M4HPTANE & 28 & 7.99 & $2.38 \mathrm{E}-59$ & $6.72 \mathrm{E}+58$ \\
\hline Benzaldehyde & BENZAL & 29 & 7.99 & \multicolumn{2}{|c|}{ Removed from analysis } \\
\hline Nonane & NNANE & 30 & 7.97 & 4.70E-60 & $3.40 \mathrm{E}+59$ \\
\hline cis-Piperylene & CPIPERLNE & 31 & 7.88 & $1.27 \mathrm{E}-59$ & $1.26 \mathrm{E}+59$ \\
\hline cis-3-Hexen-1-ol & CHX1E3OL & 32 & 7.87 & \multicolumn{2}{|c|}{ Removed from analysis. } \\
\hline Butyl Prop-2-enoate & BTESTPPEA & 33 & 7.86 & $5.45 \mathrm{E}-66$ & $4.13 \mathrm{E}+65$ \\
\hline 2.3-Dimethyl-Heptane & DM23HPTANE & 34 & 7.81 & $3.98 \mathrm{E}-66$ & $5.66 \mathrm{E}+65$ \\
\hline Ethylene Glycol Ethyl Ether & ENGOLEETHR & 35 & 7.79 & $1.29 \mathrm{E}-59$ & $1.24 \mathrm{E}+59$ \\
\hline Ethyl-Benzene & ETBNZ & 36 & 7.66 & \multicolumn{2}{|c|}{ Removed from analysis. } \\
\hline Phenol & PHEOL & 37 & 7.64 & \multicolumn{2}{|c|}{ Removed from analysis. } \\
\hline Pseudocumene & PSEUDCUMNE & 38 & 7.59 & \multicolumn{2}{|c|}{ Removed from analysis. } \\
\hline 5-Methyl-2-Heptanone & M5HPTAONE & 39 & 7.54 & \multicolumn{2}{|c|}{ Removed from analysis. } \\
\hline m-tert-Butyl-Toluene & MTBTLNE & 40 & 7.27 & \multicolumn{2}{|c|}{ Removed from analysis. } \\
\hline Ethanal & ETAL & 41 & 7.26 & 3.09E-66 & $7.28 \mathrm{E}+65$ \\
\hline 3-Ethyl-Benzaldehyde & E3BNZAL & 42 & 7.26 & \multicolumn{2}{|c|}{ Removed from analysis. } \\
\hline 5-Methyl-Decane & M5DCANE & 43 & 7.24 & \multicolumn{2}{|c|}{ Removed from analysis. } \\
\hline 1-Penten-3-ol & PT1E3OL & 44 & 7.22 & $2.94 \mathrm{E}-59$ & $5.45 \mathrm{E}+58$ \\
\hline Dihydromyrcenol & DHMYRCNOL & 45 & 7.15 & \multicolumn{2}{|c|}{ Removed from analysis. } \\
\hline 1-Pentene & PT1ENE & 46 & 7.13 & \multicolumn{2}{|c|}{ Removed from analysis. } \\
\hline$\beta$-Phellandrene & BPHELDNE & 47 & 7.12 & 2.07E-59 & $7.74 \mathrm{E}+58$ \\
\hline 3-Methyl-Butanal & M3BTAL & 48 & 7.11 & $1.67 \mathrm{E}-59$ & $9.58 \mathrm{E}+58$ \\
\hline 2.2-Dimethyl-3-Heptanone & DM22HPT3ONE & 49 & 6.91 & \multicolumn{2}{|c|}{ Removed from analysis. } \\
\hline 2-Cyclopenten-1-one & CY2PT1EONE & 50 & 6.91 & \multicolumn{2}{|c|}{ Removed from analysis. } \\
\hline 3-Methyl-1-Butanol & M3BT1OL & 51 & 6.89 & 7.19E-59 & $2.22 \mathrm{E}+58$ \\
\hline Dimethyl Sulfide & DMSULFI & 52 & 6.80 & \multicolumn{2}{|c|}{ Removed from analysis. } \\
\hline
\end{tabular}


${ }^{1} \mathrm{~F}$ - Fischer value from One-way ANOVA test.

${ }^{2} \mathrm{~W}$ - Wilks value from Linear Discriminant Analysis.

${ }^{3} \mathrm{~F}$ - Fischer value from Linear Discriminant Analysis.

${ }^{4} \mathrm{CDF}$ - Canonical Discriminant Function Coefficients. 